\section{Performance of the ALOGPS 2.1 program for octanol-water partition coefficient prediction with organic chemicals on the Canadian Domestic Substances List}

\section{Sierra Rayne ${ }^{a, *}$ and Kaya Forest ${ }^{b}$}

Octanol-water partition coefficients $\left(\mathrm{K}_{\mathrm{ow}}\right)$ are widely used in environmental and biological chemistry to predict and understand the partitioning behavior of neutral organic compounds and their salts into a range of organic materials. Because of the time and expense in obtaining experimental $\mathrm{K}_{\text {ow }}$ data, a large number of freely available and commercial $\mathrm{K}_{\mathrm{ow}}$ prediction software programs are available. One of the most popular $\mathrm{K}_{\text {ow }}$ prediction programs is KOWWIN, currently included as part of the EPI Suite environmental modeling program (http://www.epa.gov/oppt/exposure/pubs/episuitedl.htm). KOWWIN estimated $K_{\text {ow }}$ values are used on the Domestic Substances List (DSL; http://www.ec.gc.ca/ceparegistry/Subs_list/Domestic.cfm) of Environment Canada for screening level assessments of bioaccumulation potential. In the current study, we examined the predictive capacity of KOWWIN against the ALOGPS 2.1 program (http://www.vcclab.org/lab/alogps/; ref. ${ }^{[1-4]}$ ) - both freely available internet based programs - for substances on the Canadian DSL with and without comparative experimental $\mathrm{K}_{\mathrm{ow}}$ values.

From an initial sample of 1771 experimental log $\mathrm{K}_{\text {ow }}$ values in the Canadian DSL listing, the removal of duplicate entries, errors, salts, and organosilicon compounds resulted in a reduced set of 1545 compounds (Appendix Table 1). The substantial number of errors and duplicates in the original source Canadian DSL experimental $\mathrm{K}_{\text {ow }}$ list necessitates rigorous pre-screening by researchers prior to use. The following three general categories of concern were encountered: (1) duplicate entries, often from different common names and IUPAC
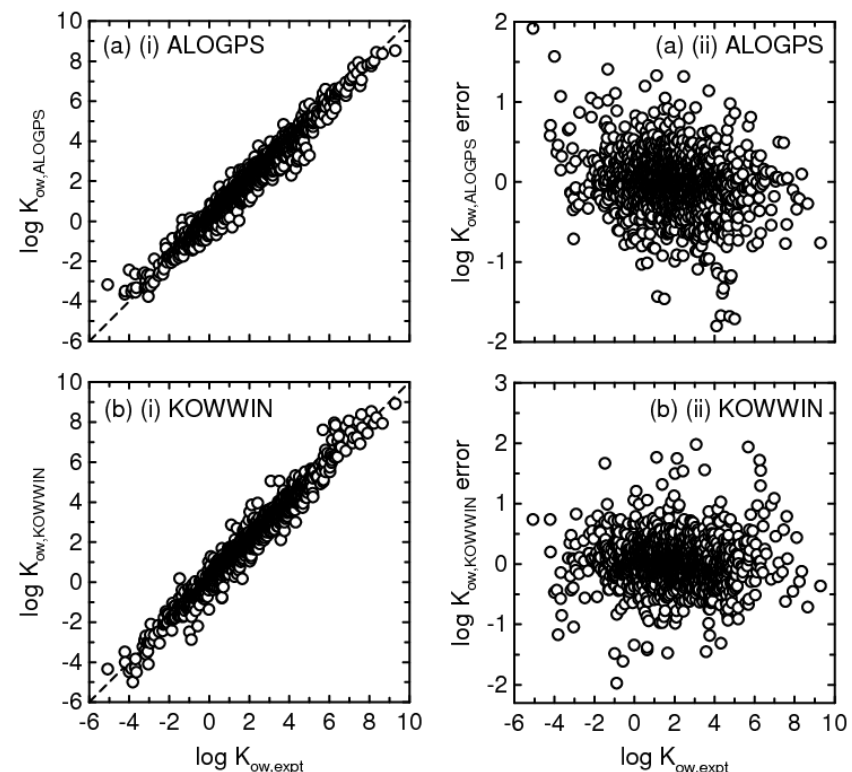

Fig. 1. Comparison between ALOGPS ((a)(i)) and KOWWIN ((b)(i)) predicted and experimental log $\mathrm{K}_{\mathrm{ow}}$ values for compounds on the Canadian DSL and corresponding distributions of residual errors for the ALOGPS ((a)(ii)) and KOWWIN ((b)(ii)) estimates. 1:1 lines (dashed) are also given. naming formalisms that all refer to the same structure; (2) failure to properly specify geometrical isomerism (i.e., cis-/trans- and E-/Zdesignation of various functional groups) or stereochemistry (i.e. $\mathrm{R} / \mathrm{S}$ ), both of which could have been designated using the SMILES notation ${ }^{[5,6]}$ in this database; and (3) while the compound name suggests the log $\mathrm{K}_{\mathrm{ow}}$ value was determined/estimated for a salt, the SMILES formula indicates the molecular form, thereby making direct comparisons between experimental and estimated data invalid, and the use of the estimated data subject to significant uncertainty. For estimated $\mathrm{K}_{\text {ow }}$ values, neither KOWWIN nor ALOGPS distinguishes between $\mathrm{E}$-/Z-isomer or diastereomer based differences in physicochemical properties.

The mean signed error (MSE), mean absolute error (MAE), and root mean squared error (RMSE) between experimental and estimated $\log \mathrm{K}_{\text {ow }}$ values for KOWWIN on these 1545 compounds were 0.02 , 0.26 , and 0.37 units, respectively, compared to $-0.02,0.25$, and 0.35 units, respectively for ALOGPS. By comparison, COSMOfrag was reported to have a RMSE of $0.74 \log \mathrm{K}_{\text {ow }}$ units using an unscreened version of the Canadian DSL. ${ }^{[7]}$ KOWWIN and ALOGPS yield the following respective regression equations between predicted and experimental log $\mathrm{K}_{\mathrm{ow}}$ values (Figure 1):

$\log \mathrm{K}_{\text {ow,ALOGPS }}=0.961( \pm 0.004) \times \log \mathrm{K}_{\text {ow,expt }}+0.058( \pm 0.012) \ldots(1)$

$n=1545, r=0.983, s=0.346, c v=0.182$

$\log \mathrm{K}_{\text {ow, Kowwin }}=0.992( \pm 0.005) \times \log \mathrm{K}_{\text {ow,expt }}+0.033( \pm 0.013) \ldots(2)$

$n=1545, r=0.982, s=0.368, c v=0.191$

Log $\mathrm{K}_{\text {ow }}$ error residuals for KOWWIN are evenly distributed with no significant trend $\left(r=-0.04, p_{m=0}=0.12, p_{b=0}=0.01\right)$. ALOGPS error residuals do display a significant trend $\left(r=-0.21, p_{m=0}<10^{-17}, p_{b=0}<10^{-5}\right)$, generally decreasing in signed error magnitude with increasing log $\mathrm{K}_{\mathrm{ow}}$.

Compounds with $\log \mathrm{K}_{\text {ow }}$ values $>5$ are often classified by regulatory agencies as having high bioaccumulation potential, provided they are sufficiently persistent in environmental and biological systems. Similarly, "Lipinski's Rule of Five" postulates that above a $\log \mathrm{K}_{\text {ow }}$ of 5 , poor absorption or permeation is anticipated in a drug discovery setting ${ }^{[8]}$ Of the 83 compounds on our screened version of the Canadian DSL with experimental $\log \mathrm{K}_{\mathrm{ow}}>5$, KOWWIN correctly classified 75 and ALOGPS correctly classified 72. KOWWIN generated 11 false positives (i.e., predicting $\log \mathrm{K}_{\mathrm{ow}}>5$ when experimental $\log \mathrm{K}_{\mathrm{ow}}<5$ ) and 8 false negatives (i.e., predicting log $\mathrm{K}_{\mathrm{ow}}<5$ when experimental $\log \mathrm{K}_{\mathrm{ow}}>5$ ). ALOGPS generated 8 false positives and 11 false negatives.

The Canadian DSL gives 9544 compounds without experimental log $\mathrm{K}_{\text {ow }}$ values, and for which KOWWIN estimates have been generated. A rudimentary screening of this list to remove duplicate entries, errors, salts, and organosilicon compounds resulted in a reduced set of 6529 compounds. A comparison of KOWWIN and ALOGPS estimated log $\mathrm{K}_{\mathrm{ow}}$ values for these compounds indicates significant predictive differences between the two programs (Fig. 2), yielding a root mean squared deviation between the methods of $2.18 \log \mathrm{K}_{\mathrm{ow}}$ units. Modest agreement exists between the programs at estimated $\log \mathrm{K}_{\text {ow }}$ values from -5 to 5 , but outside of this range the deviations between the two methods increase up to a maximum difference of $39.3 \log \mathrm{K}_{\text {ow }}$ units. Of particular interest, there are some compounds whose ALOGPS log $\mathrm{K}_{\mathrm{ow}}$ estimates are $<5$, but the corresponding KOWWIN log $K_{\text {ow }}$ estimates can range up to $>28$. 


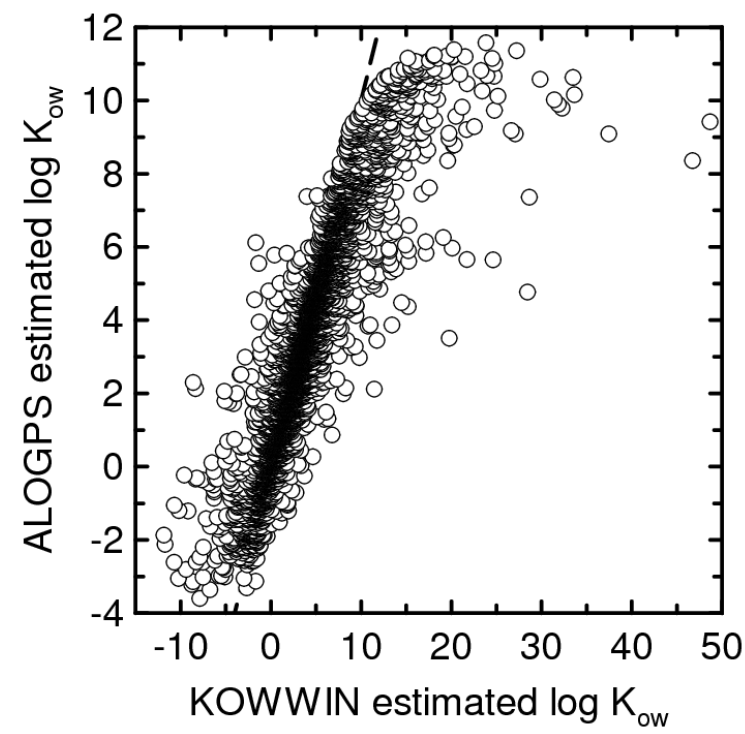

Fig. 2. Comparison between ALOGPS and KOWWIN estimated log $\mathrm{K}_{\mathrm{ow}}$ values for compounds on the Canadian DSL without experimental $\mathrm{K}_{\text {ow }}$ data. A 1:1 line (dashed) is also given.

In some cases, ALOGPS estimates a log $\mathrm{K}_{\mathrm{ow}}>5$, whereas KOWWIN gives a corresponding log $\mathrm{K}_{\text {ow }}$ estimate of less than -1 . Consequently, the differences between the two programs are not restricted to log $\mathrm{K}_{\mathrm{ow}}$ values that are clear environmental regulatory and/or drug discovery outliers (i.e., where both methods predict $\log \mathrm{K}_{\mathrm{ow}}>10$ or $<0$ ), but also for a significant number of compounds where one method predicts a value well below a log $\mathrm{K}_{\text {ow }}$ of 5 , and the other method predicts a value well above this cutoff, and vice versa.

\section{References and Notes}

${ }^{a}$ Ecologica Research, Penticton, British Columbia, Canada

${ }^{\mathrm{b}}$ Department of Chemistry, Okanagan College, Penticton, British

Columbia, Canada

* Corresponding author: rayne.sierra@gmail.com

[1] Tetko, I.V.; Bruneau, P. "Application of ALOGPS to predict 1octanol/water distribution coefficients, $\log P$, and $\log D$, of AstraZeneca in-house database." J. Pharm. Sci. 2004, 93, 31033110.

[2] Tetko, I.V.; Poda, G.I. "Application of ALOGPS 2.1 to predict log D distribution coefficient for Pfizer proprietary compounds." J. Med.

Chem. 2004, 47, 5601-5604.

[3] Tetko, I.V. "Computing chemistry on the web." Drug Discov. Today 2005, 10, 1497-1500.

[4] Tetko, I.V.; Gasteiger, J.; Todeschini, R.; Mauri, A.; Livingstone, D.; Ertl, P.; Palyulin, V.A.; Radchenko, E.V.; Zefirov, N.S.; Makarenko,

A.S.; Tanchuk, V.Y.; Prokopenko, V.V. "Virtual computational chemistry laboratory: Design and description." J. Comput. Aided Mol. Des. 2005, 19, 453-463.

[5] Weininger, D. "SMILES: A chemical language and information system. 1 . Introduction to methodology and encoding rules." $J$. Chem. Inf. Comp. Sci. 1988, 28, 31-36.

[6] Weininger, D.; Weininger, A.; Weininger, J.L. "SMILES. 2. Algorithm for generation of unique SMILES notation." J. Chem. Inf. Comp. Sci. 1989, 29, 97-101.

[7] Wittekindt, C.; Goss, K.U. "Screening the partition behavior of a large number of chemicals with a quantum-chemical software." Chemosphere 2009, 76, 460-464.
[8] Lipinski, C.A.; Lombardo, F.; Dominy, B.W.; Feeney, P.J. "Experimental and computational approaches to estimate solubility and permeability in drug discovery and development settings." Adv. Drug Deliv. Rev. 2001, 46, 3-26.

\section{Acknowledgements}

We are very much indebted to Dr. Igor Tetko at the Helmholtz Zentrum Muenchen Institute for Bioinformatics and Systems Biology (http://www.vcclab.org/ itetko/) for the substantial efforts he made critically analyzing and correcting components of our original submission, as well as his assistance in use of the OCHEM Online Chemical Database with Modeling Environment (http://ochem.eu/). 
Appendix Table 1. Comparison between experimentally obtained log $\mathrm{K}_{\mathrm{ow}}$ values and corresponding KOWWIN and ALOGPS estimates and errors.

\begin{tabular}{|c|c|c|c|c|c|c|}
\hline \multirow[b]{2}{*}{ compound } & \multirow[b]{2}{*}{ SMILES } & \multirow[b]{2}{*}{ expt } & \multicolumn{2}{|c|}{ KOWWIN } & \multicolumn{2}{|c|}{ ALOGPS } \\
\hline & & & estimate & error & estimate & error \\
\hline cystine & $\mathrm{O}=\mathrm{C}(\mathrm{O}) \mathrm{C}(\mathrm{N}) \operatorname{CsscC}(\mathrm{N}) \mathrm{C}(=\mathrm{O}) \mathrm{O}$ & -5.08 & -4.34 & 0.74 & -3.16 & 1.92 \\
\hline ornithine & $\mathrm{O}=\mathrm{C}(\mathrm{O}) \mathrm{C}(\mathrm{N}) \mathrm{CCCN}$ & -4.22 & -3.48 & 0.74 & -3.64 & 0.58 \\
\hline arginine & $\mathrm{O}=\mathrm{C}(\mathrm{O}) \mathrm{C}(\mathrm{N}) \operatorname{CcCNC}(=\mathrm{N}) \mathrm{N}$ & -4.20 & -4.00 & 0.20 & -3.49 & 0.71 \\
\hline glycine, N-(phosphonomethyl)- & $O C(=O) \operatorname{CNCP}(O)(O)=0$ & -4.00 & -4.47 & -0.47 & -2.43 & 1.57 \\
\hline aspartic acid & $\mathrm{O}=\mathrm{C}(\mathrm{O}) \mathrm{C}(\mathrm{N}) \mathrm{CC}(=\mathrm{O}) \mathrm{O}$ & -3.89 & -4.32 & -0.43 & -3.52 & 0.37 \\
\hline asparagine & $\mathrm{O}=\mathrm{C}(\mathrm{O}) \mathrm{C}(\mathrm{N}) \mathrm{CC}(=\mathrm{O}) \mathrm{N}$ & -3.82 & -4.99 & -1.17 & -3.36 & 0.46 \\
\hline alpha-glucopyranoside,-fructofuranosyl & $\begin{array}{l}\mathrm{O}(\mathrm{C}(\mathrm{C}(\mathrm{O}) \mathrm{C}(\mathrm{O}) \mathrm{C} 1 \mathrm{O}) \mathrm{CO}) \mathrm{C} 1 \mathrm{OC}(\mathrm{OC}(\mathrm{C} \\
2 \mathrm{O}) \mathrm{CO})(\mathrm{C} 2 \mathrm{O}) \mathrm{CO}\end{array}$ & -3.70 & -4.27 & -0.57 & -2.63 & 1.07 \\
\hline glutamic acid & $\mathrm{O}=\mathrm{C}(\mathrm{O}) \mathrm{C}(\mathrm{N}) \mathrm{CCC}(=\mathrm{O}) \mathrm{O}$ & -3.69 & -3.83 & -0.14 & -3.54 & 0.15 \\
\hline glutamine & $\mathrm{O}=\mathrm{C}(\mathrm{O}) \mathrm{C}(\mathrm{N}) \operatorname{CCC}(=\mathrm{O}) \mathrm{N}$ & -3.64 & -4.49 & -0.85 & -3.32 & 0.32 \\
\hline histidine & $\mathrm{O}=\mathrm{C}(\mathrm{O}) \mathrm{C}(\mathrm{N}) \mathrm{CC}(\mathrm{N}=\mathrm{CN} 1)=\mathrm{C} 1$ & -3.32 & -3.22 & 0.10 & -2.67 & 0.65 \\
\hline glucose & $\mathrm{OCC} 1 \mathrm{C}(\mathrm{O}) \mathrm{C}(\mathrm{O}) \mathrm{C}(\mathrm{O}) \mathrm{C}(\mathrm{O}) \mathrm{O} 1$ & -3.24 & -2.89 & 0.35 & -2.57 & 0.67 \\
\hline glycine & $\mathrm{O}=\mathrm{C}(\mathrm{O}) \mathrm{CN}$ & -3.21 & -3.41 & -0.20 & -3.34 & -0.13 \\
\hline butanoic acid, 4-amino- & $\mathrm{O}=\mathrm{C}(\mathrm{O}) \mathrm{CCCN}$ & -3.17 & -3.60 & -0.43 & -2.99 & 0.18 \\
\hline proline, 4-hydroxy- & $\mathrm{O}=\mathrm{C}(\mathrm{O}) \mathrm{C}(\mathrm{NCC} 1 \mathrm{O}) \mathrm{C} 1$ & -3.17 & -2.69 & 0.48 & -3.31 & -0.14 \\
\hline mannitol & $\mathrm{OCC}(\mathrm{O}) \mathrm{C}(\mathrm{O}) \mathrm{C}(\mathrm{O}) \mathrm{C}(\mathrm{O}) \mathrm{CO}$ & -3.10 & -3.01 & 0.09 & -2.68 & 0.42 \\
\hline serine & $\mathrm{O}=\mathrm{C}(\mathrm{O}) \mathrm{C}(\mathrm{N}) \mathrm{CO}$ & -3.07 & -3.46 & -0.39 & -3.42 & -0.35 \\
\hline alanine & $\mathrm{O}=\mathrm{C}(\mathrm{O}) \mathrm{CCN}$ & -3.05 & -4.09 & -1.04 & -3.26 & -0.21 \\
\hline lysine & $\mathrm{O}=\mathrm{C}(\mathrm{O}) \mathrm{C}(\mathrm{N}) \mathrm{CCCCN}$ & -3.05 & -2.99 & 0.06 & -3.76 & -0.71 \\
\hline alanine & $\mathrm{O}=\mathrm{C}(\mathrm{O}) \mathrm{C}(\mathrm{N}) \mathrm{C}$ & -2.96 & -2.99 & -0.03 & -3.05 & -0.09 \\
\hline hexanoic acid, 6-amino- & $\mathrm{O}=\mathrm{C}(\mathrm{O}) \mathrm{CCCCCN}$ & -2.95 & -2.62 & 0.33 & -2.69 & 0.26 \\
\hline threonine & $\mathrm{O}=\mathrm{C}(\mathrm{O}) \mathrm{C}(\mathrm{N}) \mathrm{C}(\mathrm{O}) \mathrm{C}$ & -2.94 & -3.04 & -0.10 & -3.01 & -0.07 \\
\hline glycine, N-glycyl- & $\mathrm{O}=\mathrm{C}(\mathrm{NCC}(=\mathrm{O}) \mathrm{O}) \mathrm{CN}$ & -2.92 & -2.09 & 0.83 & -3.23 & -0.31 \\
\hline glycine, N-methyl- & $\mathrm{O}=\mathrm{C}(\mathrm{O}) \mathrm{CNC}$ & -2.78 & -2.94 & -0.16 & -3.06 & -0.28 \\
\hline hydrazinecarboxamide & $\mathrm{NC}(=\mathrm{O}) \mathrm{NN}$ & -2.75 & -2.64 & 0.11 & -1.88 & 0.87 \\
\hline proline & $\mathrm{O}=\mathrm{C}(\mathrm{O}) \mathrm{C}(\mathrm{NCC} 1) \mathrm{C} 1$ & -2.54 & -2.15 & 0.39 & -2.71 & -0.17 \\
\hline cytidine & $\begin{array}{l}\mathrm{O}=\mathrm{C} 1 \mathrm{~N}=\mathrm{C}(\mathrm{N}) \mathrm{C}=\mathrm{CN} 1 \mathrm{C} 2 \mathrm{OC}(\mathrm{CO}) \mathrm{C}(\mathrm{O}) \\
\mathrm{C} 2 \mathrm{O}\end{array}$ & -2.51 & -2.46 & 0.05 & -2.18 & 0.33 \\
\hline cysteine & $\mathrm{O}=\mathrm{C}(\mathrm{O}) \mathrm{C}(\mathrm{N}) \mathrm{CS}$ & -2.49 & -3.05 & -0.56 & -2.57 & -0.08 \\
\hline ribose & OCC $10 \mathrm{C}(\mathrm{O}) \mathrm{C}(\mathrm{O}) \mathrm{C} 10$ & -2.32 & -2.43 & -0.11 & -2.65 & -0.33 \\
\hline 1,2,3,4-butanetetrol & $\mathrm{OCC}(\mathrm{O}) \mathrm{C}(\mathrm{O}) \mathrm{CO}$ & -2.29 & -2.11 & 0.18 & -2.03 & 0.26 \\
\hline valine & $\mathrm{C}(\mathrm{N}) \mathrm{C}(\mathrm{C}) \mathrm{C}$ & -2.26 & -2.08 & 0.18 & -2.29 & -0.03 \\
\hline tyrosine & $c(\operatorname{ccc}(0) c 1) c 1$ & -2.26 & -1.76 & 0.50 & -2.39 & -0.13 \\
\hline 1H-purine-2,6,8(3H)-trione, 7,9-dihydro- & $\mathrm{O}=\mathrm{C} 1 \mathrm{NC}(=\mathrm{O}) \mathrm{NC} 2=\mathrm{C} 1 \mathrm{NC}(=\mathrm{O}) \mathrm{N} 2$ & -2.17 & -1.46 & 0.71 & -1.12 & 1.05 \\
\hline benzenesulfonic acid, 4-amino- & $\mathrm{O}=\mathrm{S}(=\mathrm{O})(\mathrm{O}) \mathrm{c}(\operatorname{ccc}(\mathrm{N}) \mathrm{c} 1) \mathrm{c} 1$ & -2.16 & -2.08 & 0.08 & -1.35 & 0.81 \\
\hline norvaline & $\mathrm{O}=\mathrm{C}(\mathrm{O}) \mathrm{C}(\mathrm{N}) \mathrm{CCC}$ & -2.11 & -2.01 & 0.10 & -2.04 & 0.07 \\
\hline urea & $\mathrm{O}=\mathrm{C}(\mathrm{N}) \mathrm{N}$ & -2.11 & -1.56 & 0.55 & -1.78 & 0.33 \\
\hline 1,2-ethanediamine & $\mathrm{NCCN}$ & -2.04 & -1.62 & 0.42 & -1.77 & 0.27 \\
\hline uridine & $\begin{array}{l}\mathrm{O}=\mathrm{C}(\mathrm{N}(\mathrm{C}=\mathrm{CC} 1(=\mathrm{O})) \mathrm{C}(\mathrm{OC}(\mathrm{C} 2 \mathrm{O}) \mathrm{CO}) \\
\mathrm{C} 2 \mathrm{O}) \mathrm{N} 1\end{array}$ & -1.98 & -1.86 & 0.12 & -1.84 & 0.14 \\
\hline guanosine & $\begin{array}{l}\mathrm{c} 1 \mathrm{nc} 2 \mathrm{C}(=\mathrm{O}) \mathrm{NC}(\mathrm{N})=\mathrm{Nc} 2 \mathrm{n} 1 \mathrm{C} 3 \mathrm{OC}(\mathrm{CO}) \\
\mathrm{C}(\mathrm{O}) \mathrm{C} 3 \mathrm{O}\end{array}$ & -1.90 & -1.71 & 0.19 & -2.06 & -0.16 \\
\hline methionine & $\mathrm{O}=\mathrm{C}(\mathrm{O}) \mathrm{C}(\mathrm{N}) \mathrm{CCSC}$ & -1.87 & -2.41 & -0.54 & -1.85 & 0.02 \\
\hline ascorbic acid & $\mathrm{O} 1 \mathrm{C}(=\mathrm{O}) \mathrm{C}(\mathrm{O})=\mathrm{C}(\mathrm{O}) \mathrm{C} 1 \mathrm{C}(\mathrm{O}) \mathrm{CO}$ & -1.85 & -1.88 & -0.03 & -1.58 & 0.27 \\
\hline inosine & $\begin{array}{l}\mathrm{OCC} 1 \mathrm{OC}(\mathrm{C}(\mathrm{O}) \mathrm{C} 1 \mathrm{O}) \mathrm{n} 2 \mathrm{cnc} 3 \mathrm{C}(=\mathrm{O}) \mathrm{NC} \\
=\mathrm{Nc} 23\end{array}$ & -1.85 & -1.84 & 0.01 & -1.87 & -0.02 \\
\hline 2,4,5,6(1H,3H)-pyrimidinetetrone & $\mathrm{O}=\mathrm{C}(\mathrm{NC}(=\mathrm{O}) \mathrm{C}(=\mathrm{O}) \mathrm{C} 1(=\mathrm{O})) \mathrm{N} 1$ & -1.84 & -1.04 & 0.80 & -0.85 & 0.99 \\
\hline urea, hydroxy- & $\mathrm{NC}(=\mathrm{O}) \mathrm{NO}$ & -1.80 & -1.68 & 0.12 & -1.83 & -0.03 \\
\hline cytidine, 2-deoxy- & $\begin{array}{l}\mathrm{N} 1(\mathrm{C} 2 \mathrm{OC}(\mathrm{CO}) \mathrm{C}(\mathrm{O}) \mathrm{C} 2) \mathrm{C}(=\mathrm{O}) \mathrm{N}=\mathrm{C}(\mathrm{N}) \\
\mathrm{C}=\mathrm{C} 1\end{array}$ & -1.77 & -1.79 & -0.02 & -1.93 & -0.16 \\
\hline $1,2,3-p$ & $\mathrm{OCC}(\mathrm{O}) \mathrm{CO}$ & -1.76 & -1.65 & 0.11 & -1.93 & -0.17 \\
\hline 4H-imidazol-4-one, 2-amino-1,5-dihydro-1-methyl- & $\mathrm{O}=\mathrm{C}(\mathrm{NC}(=\mathrm{N}) \mathrm{N} 1 \mathrm{C}) \mathrm{C} 1$ & -1.76 & -1.21 & 0.55 & -1.65 & 0.11 \\
\hline 2(1H)-pyrimidinone, 4-amino- & $\mathrm{N} 1 \mathrm{C}(=\mathrm{O}) \mathrm{N}=\mathrm{C}(\mathrm{N}) \mathrm{C}=\mathrm{C} 1$ & -1.73 & -1.47 & 0.26 & -1.53 & 0.20 \\
\hline inosine, 2-deoxy- & $\begin{array}{l}\mathrm{OCC} 1 \mathrm{OC}(\mathrm{CC} 1 \mathrm{O}) \mathrm{n} 2 \mathrm{cnc} 3 \mathrm{C}(=\mathrm{O}) \mathrm{NC}=\mathrm{N} \\
\mathrm{c} 23\end{array}$ & -1.71 & -1.18 & 0.53 & -1.67 & 0.04 \\
\hline isoleucine & $\mathrm{O}=\mathrm{C}(\mathrm{O}) \mathrm{C}(\mathrm{N}) \mathrm{C}(\mathrm{CC}) \mathrm{C}$ & -1.70 & -1.59 & 0.11 & -1.73 & -0.03 \\
\hline 1,3-propanediol, 2,2-bis(hydroxymethyl)- & $\mathrm{OCC}(\mathrm{CO})(\mathrm{CO}) \mathrm{CO}^{\prime}$ & -1.69 & -1.77 & -0.08 & -1.92 & -0.23 \\
\hline acetamide, n-hydroxy- & $\mathrm{CC}(=\mathrm{O}) \mathrm{NO}$ & -1.59 & -1.29 & 0.30 & -1.46 & 0.13 \\
\hline $\begin{array}{l}\text { 6H-purin-6-one, 2-amino-1,9-dihydro-9-[(2- } \\
\text { hydroxyethoxy)methyl]- }\end{array}$ & $\mathrm{N} 1 \mathrm{C}(\mathrm{N})=\mathrm{Nc} 2 \mathrm{n}(\mathrm{COCCO}) \mathrm{cnc} 2 \mathrm{C} 1=\mathrm{O}$ & -1.56 & -1.70 & -0.14 & -1.45 & 0.11 \\
\hline leucine & $\mathrm{O}=\mathrm{C}(\mathrm{O}) \mathrm{C}(\mathrm{N}) \mathrm{CC}(\mathrm{C}) \mathrm{C}$ & -1.52 & -1.59 & -0.07 & -1.82 & -0.30 \\
\hline
\end{tabular}


formamide

uridine, 2-deoxy-

piperazine

1,3-propanediol, 2-ethyl-2-(hydroxymethyl)-

2,4,6(1H,3H,5H)-pyrimidinetrione

riboflavin

glucose, 2-deoxy-2-

[[(methylnitrosoamino)carbonyl]amino]-

phenylalanine

ethanol, 2,2-iminobis-

1,4-piperazinedipropanamine

1,3-propanediamine

methane, sulfonylbis-

1,2-benzenediol, 4-[1-hydroxy-2-

(methylamino)ethyl]-

1,3,5-triazine-2,4,6-triamine

1,2-ethanediol

methane, sulfinylbis-

2,3-butanedione

propanenitrile, 3,3-iminobis-

ethanol, 2-amino-

cytidine, 2,3-dideoxy-

guanosine, 2-deoxy-

butanedioic acid, hydroxy-

galactopyranoside, 1-methylethyl 1-thio-

acetamide

1,2-benzenediol, 4-[2-amino-1-hydroxyethyl]-

inosine, 2,3-dideoxy-

glucopyranoside, 2-(hydroxymethyl)phenyl

benzenesulfonamide, 4-amino-N-

(aminoiminomethyl)-

1-naphthalenesulfonic acid, 2-amino-

guanidine, cyano-

6H-purin-6-one, 1,7-dihydro-

acetic acid, hydroxy-

thiourea

2,4(1H,3H)-pyrimidinedione

tryptophan

adenosine

tryptophan

acetamide, N-methyl-

1,3-propanediol

3-quinolinecarboxylic acid, 1-ethyl-6-fluoro-1,4-

dihydro-4-oxo-7-(1-piperazinyl)-

formamide, $\mathrm{N}, \mathrm{N}$-dimethyl-

ethanol, 2,2,2-nitrilotris-

uridine, 2 ,3-dideoxy-

butanedinitrile

formamide, N-methyl-

benzenepropanoic acid, beta-(aminomethyl)-4chloro-

2-propanol, 1-amino-

acetamide, N-[(4-aminophenyl)sulfonyl]-

oxiranemethanol

2,5-pyrrolidinedione, 1-methyl-

ethanol, 2-(methylamino)-

propanenitrile, 3-hydroxy-

thymidine

1,2-propanediol

\begin{tabular}{|c|c|c|c|c|c|}
\hline $\mathrm{O}=\mathrm{CN}$ & -1.51 & -1.61 & -0.10 & -1.53 & -0.02 \\
\hline $\begin{array}{l}\mathrm{O}=\mathrm{C}(\mathrm{N}(\mathrm{C}=\mathrm{CC} 1(=\mathrm{O})) \mathrm{C}(\mathrm{OC}(\mathrm{C} 2 \mathrm{O}) \mathrm{CO}) \\
\mathrm{C} 2) \mathrm{N} 1\end{array}$ & -1.51 & -1.19 & 0.32 & -1.49 & 0.02 \\
\hline $\mathrm{N}(\mathrm{CCNC} 1) \mathrm{C} 1$ & -1.50 & -0.80 & 0.70 & -1.16 & 0.34 \\
\hline $\mathrm{OCC}(\mathrm{CC})(\mathrm{CO}) \mathrm{CO}$ & -1.48 & 0.19 & 1.67 & -0.76 & 0.72 \\
\hline $\mathrm{O}=\mathrm{C}(\mathrm{NC}(=\mathrm{O}) \mathrm{CC} 1(=\mathrm{O})) \mathrm{N} 1$ & -1.47 & -1.25 & 0.22 & -1.12 & 0.35 \\
\hline $\begin{array}{l}\mathrm{Cc} 1 \mathrm{c}(\mathrm{C}) \mathrm{cc} 2 \mathrm{~N}=\mathrm{C} 3 \mathrm{C}(=\mathrm{O}) \mathrm{NC}(=\mathrm{O}) \mathrm{N}=\mathrm{C} 3 \\
\mathrm{~N}(\mathrm{CC}(\mathrm{O}) \mathrm{C}(\mathrm{O}) \mathrm{C}(\mathrm{O}) \mathrm{CO}) \mathrm{c} 2 \mathrm{c} 1\end{array}$ & -1.46 & -1.05 & 0.41 & -1.05 & 0.41 \\
\hline $\begin{array}{l}\mathrm{CN}(\mathrm{N}=\mathrm{O}) \mathrm{C}(=\mathrm{O}) \mathrm{NC} 1 \mathrm{C}(\mathrm{O}) \mathrm{OC}(\mathrm{CO}) \mathrm{C}(\mathrm{O} \\
) \mathrm{C} 1 \mathrm{O}\end{array}$ & -1.45 & -1.61 & -0.16 & -1.71 & -0.2 \\
\hline $\mathrm{O}=\mathrm{C}(\mathrm{O}) \mathrm{C}(\mathrm{N}) \mathrm{Cc}(\operatorname{cccc} 1) \mathrm{c} 1$ & -1.44 & -1.28 & 0.16 & -1.35 & 0.09 \\
\hline OCCNCCO & -1.43 & -1.71 & -0.28 & -1.41 & 0.02 \\
\hline $\mathrm{N}(\mathrm{CCN}(\mathrm{C} 1) \mathrm{CCCN})(\mathrm{C} 1) \mathrm{CCCN}$ & -1.43 & -1.35 & 0.08 & -0.67 & 0.76 \\
\hline $\mathrm{NCCCN}$ & -1.43 & -1.13 & 0.30 & -1.41 & 0.02 \\
\hline $\mathrm{O}=\mathrm{S}(=\mathrm{O})(\mathrm{C}) \mathrm{C}$ & -1.41 & -1.11 & 0.30 & -0.95 & 0.46 \\
\hline $\mathrm{OC}(\mathrm{c}(\mathrm{ccc}(\mathrm{O}) \mathrm{c} 1 \mathrm{O}) \mathrm{c} 1) \mathrm{CNC}$ & -1.37 & -0.69 & 0.68 & -0.82 & 0.55 \\
\hline$n(c(n c(n 1) N) N) c 1 N$ & -1.37 & -0.38 & 0.99 & -0.69 & 0.68 \\
\hline $\mathrm{OCCO}$ & -1.36 & -1.20 & 0.16 & -1.53 & -0.17 \\
\hline $\mathrm{O}=\mathrm{S}(\mathrm{C}) \mathrm{C}$ & -1.35 & -1.22 & 0.13 & -1.09 & 0.26 \\
\hline $\mathrm{O}=\mathrm{C}(\mathrm{C}(=\mathrm{O}) \mathrm{C}) \mathrm{C}$ & -1.34 & -1.34 & 0.00 & 0.07 & 1.41 \\
\hline $\mathrm{C}(\# \mathrm{~N}) \mathrm{CCNCCC}(\# \mathrm{~N})$ & -1.34 & -1.15 & 0.19 & -0.32 & 1.02 \\
\hline OCCN & -1.31 & -1.61 & -0.30 & -1.53 & -0.22 \\
\hline $\begin{array}{l}\mathrm{O} 1 \mathrm{C}(\mathrm{CO}) \mathrm{CCC} 1 \mathrm{~N} 2 \mathrm{C}(=\mathrm{O}) \mathrm{N}=\mathrm{C}(\mathrm{N}) \mathrm{C}=\mathrm{C} \\
2\end{array}$ & -1.30 & -1.72 & -0.42 & -1.29 & \\
\hline $\begin{array}{l}\mathrm{O} 1 \mathrm{C}(\mathrm{CO}) \mathrm{C}(\mathrm{O}) \mathrm{CC} 1 \mathrm{n} 2 \mathrm{c} 3 \mathrm{~N}=\mathrm{C}(\mathrm{N}) \mathrm{NC}(= \\
\mathrm{O}) \mathrm{c} 3 \mathrm{nc} 2\end{array}$ & -1.30 & -1.04 & 0.26 & -1.75 & -0.4 \\
\hline $\mathrm{O}=\mathrm{C}(\mathrm{O}) \mathrm{CC}(\mathrm{O}) \mathrm{C}(=\mathrm{O}) \mathrm{O}$ & -1.26 & -1.68 & -0.42 & -0.87 & \\
\hline $\mathrm{O} 1 \mathrm{C}(\mathrm{CO}) \mathrm{C}(\mathrm{O}) \mathrm{C}(\mathrm{O}) \mathrm{C}(\mathrm{O}) \mathrm{C} 1 \mathrm{SC}(\mathrm{C}) \mathrm{C}$ & -1.26 & -1.25 & 0.01 & -1.11 & \\
\hline $\mathrm{O}=\mathrm{C}(\mathrm{N}) \mathrm{C}$ & -1.26 & -1.16 & 0.10 & -1.10 & 0.16 \\
\hline $\mathrm{c} 1 \mathrm{cc}(\mathrm{O}) \mathrm{c}(\mathrm{O}) \mathrm{cc} 1 \mathrm{C}(\mathrm{O}) \mathrm{CN}$ & -1.24 & -1.16 & 0.08 & -1.40 & -0.16 \\
\hline $\mathrm{O} 1 \mathrm{C}(\mathrm{CO}) \mathrm{CCC} 1 \mathrm{~N} 2 \mathrm{c} 3 \mathrm{ncnc}(\mathrm{O}) \mathrm{c} 3 \mathrm{~N}=\mathrm{C} 2$ & -1.24 & -0.47 & 0.77 & -0.99 & 0.2 \\
\hline $\begin{array}{l}\mathrm{O}(\mathrm{C}(\mathrm{C}(\mathrm{O}) \mathrm{C}(\mathrm{O}) \mathrm{C} 1 \mathrm{O}) \mathrm{CO}) \mathrm{C} 1 \mathrm{Oc}(\mathrm{c}(\mathrm{ccc} 2 \\
) \mathrm{CO}) \mathrm{c} 2\end{array}$ & -1.22 & -1.41 & -0.19 & -1.15 & 0 \\
\hline $\operatorname{c1cc}(\mathrm{N}) \operatorname{ccc} 1 \mathrm{~S}(=\mathrm{O})(=\mathrm{O}) \mathrm{NC}(=\mathrm{N}) \mathrm{N}$ & -1.22 & -1.07 & 0.15 & -0.55 & 0.67 \\
\hline$(c(c(\operatorname{ccc} 1) \operatorname{cc} 2) c 1) c 2 N$ & -1.16 & -0.91 & 0.25 & -0.75 & 0.41 \\
\hline $\mathrm{NC}(=\mathrm{NC}(\# \mathrm{~N})) \mathrm{N}$ & -1.15 & -1.26 & -0.11 & -1.02 & 0.1 \\
\hline $\mathrm{N} 1 \mathrm{C}=\mathrm{Nc} 2 \mathrm{nc}[\mathrm{nH}] \mathrm{c} 2 \mathrm{C} 1(=\mathrm{O})$ & -1.11 & -1.19 & -0.08 & -1.15 & -0.0 \\
\hline $\mathrm{O}=\mathrm{C}(\mathrm{O}) \mathrm{CO}$ & -1.11 & -1.07 & 0.04 & -1.02 & 0.0 \\
\hline $\mathrm{NC}(=\mathrm{S}) \mathrm{N}$ & -1.08 & -1.31 & -0.23 & -1.07 & 0. \\
\hline $\mathrm{N} 1 \mathrm{C}(=\mathrm{O}) \mathrm{NC}(=\mathrm{O}) \mathrm{C}=\mathrm{C} 1$ & -1.07 & -0.87 & 0.20 & -1.20 & -( \\
\hline $\mathrm{O}=\mathrm{C}(\mathrm{O}) \mathrm{C}(\mathrm{N}) \mathrm{CC}(\mathrm{c}(\mathrm{c}(\mathrm{N} 1) \mathrm{ccc} 2) \mathrm{c} 2)=\mathrm{C} 1$ & -1.06 & -1.22 & -0.16 & -1.10 & -0.0 \\
\hline $\begin{array}{l}\text { n1cnc2n(C3OC }(\mathrm{CO}) \mathrm{C}(\mathrm{O}) \mathrm{C} 3(\mathrm{O})) \mathrm{cnc} 2 \\
\mathrm{c} 1 \mathrm{~N}\end{array}$ & -1.05 & -1.38 & -0.33 & -1.21 & \\
\hline $\mathrm{O}=\mathrm{C}(\mathrm{O}) \mathrm{C}(\mathrm{N}) \mathrm{Cc}(\mathrm{c}(\mathrm{c}(\mathrm{n} 1) \mathrm{ccc} 2) \mathrm{c} 2) \mathrm{c} 1$ & -1.05 & -1.22 & -0.17 & -1.10 & \\
\hline $\mathrm{O}=\mathrm{C}(\mathrm{NC}) \mathrm{C}$ & -1.05 & -0.70 & 0.35 & -1.06 & \\
\hline OCCCO & -1.04 & -0.71 & 0.33 & -1.18 & -0.1 \\
\hline $\begin{array}{l}\mathrm{C} 1 \mathrm{CNCCN} 1 \mathrm{c} 2 \mathrm{cc} 3 \mathrm{~N}(\mathrm{CC}) \mathrm{C}=\mathrm{C}(\mathrm{C}(=\mathrm{O}) \\
\mathrm{O}) \mathrm{C}(=\mathrm{O}) \mathrm{c} 3 \mathrm{cc} 2 \mathrm{~F}\end{array}$ & -1.03 & -0.31 & 0.72 & -0.47 & \\
\hline $\mathrm{O}=\mathrm{CN}(\mathrm{C}) \mathrm{C}$ & -1.01 & -0.93 & 0.08 & -0.77 & 0.2 \\
\hline $\mathrm{OCCN}(\mathrm{CCO}) \mathrm{CCO}$ & -1.00 & -2.48 & -1.48 & -1.38 & 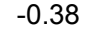 \\
\hline $\begin{array}{l}\mathrm{O} 1 \mathrm{C}(\mathrm{CO}) \mathrm{CCC} 1 \mathrm{~N} 2 \mathrm{C}(=\mathrm{O}) \mathrm{NC}(=\mathrm{O}) \mathrm{C}=\mathrm{C} \\
2\end{array}$ & -1.00 & -1.12 & -0.12 & -1.06 & \\
\hline $\mathrm{C}(\# \mathrm{~N}) \mathrm{CCC}(\# \mathrm{~N})$ & -0.99 & -0.63 & 0.36 & -0.75 & \\
\hline $\mathrm{O}=\mathrm{CNC}$ & -0.97 & -1.14 & -0.17 & -1.31 & -0.3 \\
\hline $\operatorname{c1cc}(\mathrm{Cl}) \operatorname{ccc} 1 \mathrm{C}(\mathrm{CN}) \mathrm{CC}(=\mathrm{O}) \mathrm{O}$ & -0.96 & -1.32 & -0.36 & -0.82 & 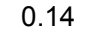 \\
\hline $\mathrm{OC}(\mathrm{CN}) \mathrm{C}$ & -0.96 & -1.1 & -0.23 & -1.03 & 0.0 \\
\hline $\mathrm{O}=\mathrm{C}(\mathrm{NS}(=\mathrm{O})(=\mathrm{O}) \mathrm{c}(\operatorname{ccc}(\mathrm{N}) \mathrm{c} 1) \mathrm{c} 1) \mathrm{C}$ & -0.96 & -0.60 & 0.36 & 0.15 & \\
\hline $\mathrm{O}(\mathrm{C} 1 \mathrm{CO}) \mathrm{C} 1$ & -0.95 & -1.09 & -0.14 & -0.93 & 0.02 \\
\hline CN1C $(=0) C C C 1(=0)$ & -0.95 & -0.64 & 0.31 & -0.70 & 0.25 \\
\hline OCCNC & -0.94 & -1.15 & -0.21 & -1.05 & -0.1 \\
\hline $\mathrm{C}(\# \mathrm{~N}) \mathrm{CCO}$ & -0.94 & -1.12 & -0.18 & -0.98 & -0.0 \\
\hline $\begin{array}{l}\mathrm{O}=\mathrm{C}(\mathrm{N}(\mathrm{C}=\mathrm{C}(\mathrm{C} 1(=\mathrm{O})) \mathrm{C}) \mathrm{C}(\mathrm{OC}(\mathrm{C} 2 \mathrm{O}) \mathrm{C} \\
\mathrm{O}) \mathrm{C} 2) \mathrm{N} 1\end{array}$ & -0.93 & -0.64 & 0.29 & -1.32 & \\
\hline $\mathrm{OCC}(\mathrm{O}) \mathrm{C}$ & -0.92 & -0.78 & 0.14 & -1.10 & \\
\hline
\end{tabular}


2,3-butanediol

6H-purin-6-one, 2-amino-1,7-dihydro-

2-naphthacenecarboxamide, 4-(dimethylamino)-

1,4,4a,5,5a,6,11,12a-octahydro-3,5,6,10,12,12a-

hexahydroxy-6-methyl-1,11-dioxo-

guanidine, nitro-

galactopyranoside, phenyl

glycine, N-(4-aminobenzoyl)-

morpholine

1H-1,2,4-triazol-3-amine

2-pyrrolidinone

3,6-pyridazinedione, 1,2-dihydro-

1,4-butanediol

4-pyrimidinecarboxylic acid, 1,2,3,6-tetrahydro-2,6dioxo-

2-propanol, 1,1-iminobis-

propanedioic acid

2-butene-1,4-diol

galactopyranoside, 2-nitrophenyl

ethanol, 2-methoxy-

methanol

3,4-pyridinedimethanol, 5-hydroxy-6-methyl-

acetamide, $\mathrm{N}, \mathrm{N}$-dimethyl-

thiophene, tetrahydro-, 1,1-dioxide

acetic acid, cyano-

1H-purine-2,6-dione, 3,7-dihydro-

propanoic acid, 2-hydroxy-

4-pyridinecarboxylic acid, hydrazide

$1 \mathrm{H}$-imidazole-4-ethanamine

1,3-propanediol, 2-(bromomethyl)-2-

(hydroxymethyl)-

$1,4,7,10,13,16$-hexaoxacyclooctadecane

2-propenamide

2-imidazolidinethione

2H-1-benzopyran-2-one, 7-galactopyranosyloxy-4-

methyl-

phosphoric acid, trimethyl ester

propanimidamide, 3-[[[2-

[(aminoiminomethyl)amino]-4-thiazolyl]methyl]thio]-

$\mathrm{N}$-(aminosulfonyl)-

2(3H)-furanone, dihydro-

ethanol, 2,2-thiobis-

benzenesulfonamide, 4-amino-

adenosine, 3-deoxy-

phosphonic acid, methyl-, dimethyl ester

propanedinitrile

thymidine, 3-deoxy-

butanedioic acid

galactopyranoside, 4-nitrophenyl

5-thia-1-azabicyclo[4.2.0]oct-2-ene-2-carboxylic acid, 3-[[(5-methyl-1,3,4-thiadiazol-2-yl)thio]methyl]-

8-oxo-7-[(1H-tetrazol-1-ylacetyl)amino]-

$1 \mathrm{H}-1,2,4$-triazole

methanamine

adenosine, 2-deoxy-

4H-pyrazolo[3,4-d]pyrimidin-4-one, 1,5-dihydroethanol, 2-(2-ethoxyethoxy)-

formic acid

acetamide, 2-chloro-
$\mathrm{OC}(\mathrm{C}(\mathrm{O}) \mathrm{C}) \mathrm{C}$

$\mathrm{N} 1 \mathrm{C}(\mathrm{N})=\mathrm{Nc} 2 \mathrm{nc}[\mathrm{nH}] \mathrm{c} 2 \mathrm{C} 1(=\mathrm{O})$

$\mathrm{O}=\mathrm{C}(\mathrm{N}) \mathrm{C}(=\mathrm{C}(\mathrm{O}) \mathrm{C}(\mathrm{N}(\mathrm{C}) \mathrm{C}) \mathrm{C}(\mathrm{C} 1(\mathrm{O}) \mathrm{C}($

$\mathrm{O})=\mathrm{C}(\mathrm{C} 2 \mathrm{C}(\mathrm{O})$

$(\mathrm{c}(\mathrm{c} 3 \mathrm{c}(\mathrm{O}) \mathrm{cc} 4) \mathrm{c} 4) \mathrm{C}) \mathrm{C} 3(=\mathrm{O})) \mathrm{C} 2 \mathrm{O}) \mathrm{C} 1($

$=\mathrm{O})$

$\mathrm{N}(=\mathrm{O})(=\mathrm{O}) \mathrm{NC}(=\mathrm{N}) \mathrm{N}$

$\mathrm{O} 1 \mathrm{C}(\mathrm{CO}) \mathrm{C}(\mathrm{O}) \mathrm{C}(\mathrm{O}) \mathrm{C}(\mathrm{O}) \mathrm{C} 1 \mathrm{Oc} 2 \mathrm{ccccc}$

2

$\mathrm{O}=\mathrm{C}(\mathrm{NCC}(=\mathrm{O}) \mathrm{O}) \mathrm{c}(\operatorname{ccc}(\mathrm{N}) \mathrm{c} 1) \mathrm{c} 1$

$\mathrm{O}(\mathrm{CCNC} 1) \mathrm{C} 1$

$\mathrm{n} 1 \mathrm{nc}(\mathrm{N}) \mathrm{nc} 1$

$\mathrm{O}=\mathrm{C}(\mathrm{NCC} 1) \mathrm{C} 1$

$\mathrm{C} 1=\mathrm{CC}(=\mathrm{O}) \mathrm{NNC} 1(=\mathrm{O})$

$\mathrm{OCCCCO}$

$\mathrm{O}=\mathrm{C} 1 \mathrm{NC}(=\mathrm{O}) \mathrm{NC}(\mathrm{C}(=\mathrm{O}) \mathrm{O})=\mathrm{C} 1$

$\mathrm{OC}(\mathrm{C}) \mathrm{CNCC}(\mathrm{O}) \mathrm{C}$

$\mathrm{O}=\mathrm{C}(\mathrm{O}) \mathrm{CC}(=\mathrm{O}) \mathrm{O}$

$\mathrm{OCC}=\mathrm{CCO}$

$\mathrm{N}(=\mathrm{O})$

$(=\mathrm{O}) \mathrm{c} 1 \mathrm{ccccc} 1 \mathrm{OC} 2 \mathrm{C}(\mathrm{O}) \mathrm{C}(\mathrm{O}) \mathrm{C}(\mathrm{O}) \mathrm{C}(\mathrm{C}$

$\mathrm{O}) \mathrm{O} 2$

$\mathrm{O}(\mathrm{CCO}) \mathrm{C}$

$\mathrm{OC}$

$\mathrm{n}(\mathrm{c}(\mathrm{c}(\mathrm{O}) \mathrm{c}(\mathrm{c} 1 \mathrm{CO}) \mathrm{CO}) \mathrm{C}) \mathrm{c} 1$

$\mathrm{O}=\mathrm{C}(\mathrm{N}(\mathrm{C}) \mathrm{C}) \mathrm{C}$

$\mathrm{O}=\mathrm{S}(=\mathrm{O})(\mathrm{CCC} 1) \mathrm{C} 1$

$\mathrm{O}=\mathrm{C}(\mathrm{O}) \mathrm{CC}(\# \mathrm{~N})$

$\mathrm{N} 1 \mathrm{C}(=\mathrm{O}) \mathrm{Nc} 2 \mathrm{nc}[\mathrm{nH}] \mathrm{c} 2 \mathrm{C} 1(=\mathrm{O})$

$\mathrm{O}=\mathrm{C}(\mathrm{O}) \mathrm{C}(\mathrm{O}) \mathrm{C}$

$\mathrm{NNC}(=\mathrm{O}) \mathrm{c} 1 \mathrm{ccncc} 1$

$\mathrm{C} 1=\mathrm{NC}=\mathrm{C}(\mathrm{CCN}) \mathrm{N} 1$

$\mathrm{OCC}(\mathrm{CBr})(\mathrm{CO}) \mathrm{CO}$

$\mathrm{O}($ Ccoccoccoccoccoc1)C1

$\mathrm{O}=\mathrm{C}(\mathrm{N}) \mathrm{C}=\mathrm{C}$

$\mathrm{N}(\mathrm{C}(=\mathrm{S}) \mathrm{NC} 1) \mathrm{C} 1$

$\mathrm{O}=\mathrm{C}(\mathrm{Oc}(\mathrm{c}(\mathrm{C}=1 \mathrm{C}) \operatorname{ccc} 2 \mathrm{OC}(\mathrm{OC}(\mathrm{C}(\mathrm{O}) \mathrm{C}$

3O)CO)C3O)c2)C1

$\mathrm{O}=\mathrm{P}(\mathrm{OC})(\mathrm{OC}) \mathrm{OC}$

$\mathrm{NS}(=\mathrm{O})$

$(=\mathrm{O}) \mathrm{N}=\mathrm{C}(\mathrm{N}) \operatorname{CCSCC} 1=\mathrm{CsC}(\mathrm{N}=\mathrm{C}(\mathrm{N})$

$\mathrm{N})=\mathrm{N} 1$

$\mathrm{O}=\mathrm{C}(\mathrm{OCC} 1) \mathrm{C} 1$

OCCSCCO

$\mathrm{O}=\mathrm{S}(=\mathrm{O})(\mathrm{N}) \mathrm{c}(\operatorname{ccc}(\mathrm{N}) \mathrm{c} 1) \mathrm{c} 1$

$\mathrm{c} 12 \mathrm{~N}=\mathrm{CN}(\mathrm{C} 3 \mathrm{OC}(\mathrm{CO}) \mathrm{CC} 3 \mathrm{O}) \mathrm{c} 1 \mathrm{ncnc} 2$ $\mathrm{N}$

$\mathrm{O}=\mathrm{P}(\mathrm{OC})(\mathrm{OC}) \mathrm{C}$

$\mathrm{C}($ \#N)CC(\#N)

$\mathrm{O} 1 \mathrm{C}(\mathrm{CO}) \mathrm{CCC} 1 \mathrm{~N} 2 \mathrm{C}(=\mathrm{O}) \mathrm{NC}(=\mathrm{O}) \mathrm{C}(\mathrm{C})$

$=\mathrm{C} 2$

$\mathrm{O}=\mathrm{C}(\mathrm{O}) \mathrm{CCC}(=\mathrm{O}) \mathrm{O}$

$\mathrm{O} 1 \mathrm{C}(\mathrm{CO}) \mathrm{C}(\mathrm{O}) \mathrm{C}(\mathrm{O}) \mathrm{C}(\mathrm{O}) \mathrm{C} 1 \mathrm{Oc} 2 \mathrm{ccc}(\mathrm{N}$ $(=0)(=0)) c c 2$

c1nnnn1CC $(=0) \mathrm{NC} 2 \mathrm{C}(=0) \mathrm{N} 3 \mathrm{C}(\mathrm{C}(=$

$\mathrm{O}) \mathrm{O})=\mathrm{C}(\mathrm{CSc} 4 \mathrm{nnc}(\mathrm{C}) \mathrm{s} 4) \mathrm{CSC} 23$

n1ncnc1

NC

c1nc2c(N)ncnc2n1C3OC(CO)C(O)C

3

c1nc(O)c2cnnc2n1

$\mathrm{O}(\mathrm{CCOCC}) \mathrm{CCO}$

$\mathrm{O}=\mathrm{CO}$

$\mathrm{O}=\mathrm{C}(\mathrm{N}) \mathrm{CCl}$
$-0.58$

$\begin{array}{lllll}-0.92 & -0.36 & 0.56 & -0.59 & 0.33\end{array}$

$\begin{array}{lllll}-0.91 & -1.05 & -0.14 & -0.97 & -0.06\end{array}$

$\begin{array}{lllll}-0.90 & -2.87 & -1.97 & -0.99 & -0.09\end{array}$

$\begin{array}{lllll}-0.89 & -1.72 & -0.83 & -0.97 & -0.08\end{array}$

$\begin{array}{lllll}-0.89 & -0.50 & 0.39 & -0.64 & 0.25\end{array}$

$\begin{array}{lllll}-0.89 & -0.46 & 0.43 & 0.00 & 0.89\end{array}$

$\begin{array}{lllll}-0.86 & -0.56 & 0.30 & -0.75 & 0.11\end{array}$

$\begin{array}{lllll}-0.86 & -0.47 & 0.39 & -0.89 & -0.03\end{array}$

$\begin{array}{lllll}-0.85 & -0.32 & 0.53 & -0.90 & -0.05\end{array}$

$\begin{array}{lllll}-0.84 & -0.89 & -0.05 & -1.44 & -0.60\end{array}$

$\begin{array}{lllll}-0.83 & -0.22 & 0.61 & -0.63 & 0.20\end{array}$

$\begin{array}{lllll}-0.83 & -0.11 & 0.72 & -0.89 & -0.06\end{array}$

$\begin{array}{lllll}-0.82 & -0.88 & -0.06 & -0.40 & 0.42\end{array}$

$\begin{array}{lllll}-0.81 & -1.25 & -0.44 & -0.60 & 0.21\end{array}$

$\begin{array}{lllll}-0.81 & -0.43 & 0.38 & -0.64 & 0.17\end{array}$

$\begin{array}{lllll}-0.78 & -0.68 & 0.10 & -0.58 & 0.20\end{array}$

$-0.77 \quad-0.91$

$-0.77 \quad-0.63$

$-0.77 \quad-0.56$

$-0.77 \quad-0.49$

$-0.77 \quad-0.24$

$-0.76 \quad-0.76$

$-0.73-1.15$

$-0.72-0.65$

$-0.70 \quad-0.81$

$-0.70 \quad-0.73$

$-0.70 \quad 0.04$

$-0.68 \quad-1.42$

$-0.67-0.81$

$-0.66 \quad-0.49$

$-0.43$

$-0.65-0.60$

$-0.64 \quad-0.65$

$-0.14$

0.14

0.21

0.28

0.53

0.00

$-0.42$

0.07

$-0.11$

$-0.03$

0.74

$-0.74$

$-0.14$

0.17

0.23

0.05

$-0.01$

$-0.78$

$-0.01$

$-1.38 \quad-0.61$

$\begin{array}{ll}-0.57 & 0.20\end{array}$

$\begin{array}{ll}-0.59 & 0.18\end{array}$

$\begin{array}{ll}-0.65 & 0.12\end{array}$

$-0.46 \quad 0.30$
-0.65

$\begin{array}{ll}-0.65 & 0.08\end{array}$

$\begin{array}{ll}-0.79 & -0.07\end{array}$

$\begin{array}{ll}-0.71 \quad-0.01 \\ -0.88 & -0.18\end{array}$

$-0.88 \quad-0.18$

$-0.94 \quad-0.24$

$\begin{array}{ll}-0.39 & 0.29\end{array}$

$\begin{array}{ll}-0.65 & 0.02\end{array}$

$-1.07 \quad-0.41$

$\begin{array}{ll}-0.34 & 0.32\end{array}$

$-0.61 \quad 0.04$

$\begin{array}{ll}-0.20 & 0.44\end{array}$

\section{$-0.31$}

$-0.63 \quad-0.62$

$\begin{array}{ll}-0.62 & -0.55\end{array}$

0.33

0.01

0.01
0.07

$\begin{array}{ll}-0.11 & 0.53\end{array}$

$\begin{array}{ll}-0.65 & -0.02\end{array}$

$\begin{array}{ll}-0.65 & -0.02 \\ -0.16 & 0.46\end{array}$

$\begin{array}{lll}-0.30 & -0.85 & -0.24\end{array}$

0.02

$\begin{array}{ll}-0.58 & 0.03\end{array}$

$-0.61 \quad-0.59$

$-0.60-0.60$

0.00

$-0.84$

$-0.24$

$-0.26$

$-0.59$

$-0.75$

$-0.16$

$-0.53 \quad 0.06$
-0.63

$-0.63 \quad-0.04$

$-2.19$

$-1.61$

$-0.40$

0.18

$-0.76$

$-0.18$

$-0.78 \quad-0.20$

$-0.57-0.64$

$-0.07$

$-0.49$

$\begin{array}{lll}-0.16 & -0.95 & -0.40\end{array}$

$\begin{array}{lllll}-0.55 & -0.55 & 0.00 & -0.41 & 0.14\end{array}$

$\begin{array}{lllll}-0.54 & -0.69 & -0.15 & -0.16 & 0.38\end{array}$

$\begin{array}{lllll}-0.54 & -0.46 & 0.08 & -0.47 & 0.07\end{array}$

$\begin{array}{lllll}-0.53 & -0.58 & -0.05 & -0.67 & -0.14\end{array}$ 
1,4-butanediol, dimethanesulfonate urea, dimethyl-

pentanoic acid, 4-oxo-

1,4,7,10,13-pentaoxacyclopentadecane

2-butenedioic acid

4H-1-benzopyran-4-one, 7-[[2-o-(6-deoxy-alpha-Imannopyranosyl)--d-glucopyranosyl]oxy]-2,3-

dihydro-5-hydroxy-2-(4-hydroxyphenyl)-

1,3,5-trioxane

2-propyn-1-amine

benzamide, 4-amino-

1,3-dioxolan-2-one, 4-methyl-

piperazine, 1,4-dimethyl-

2-propyn-1-ol

methanamine, N-methyl-

2-pyrrolidinone, 1-methyl-

adenosine, 2-chloro-

3-pyridinecarboxamide

1,3-dioxolane

ethane, 1,1-oxybis[2-methoxy-]

propanoic acid, 2-hydroxy-2-methyl-

methane, nitro-

$2 \mathrm{H}$-imidazole-2-thione, 1,3-dihydro-1-methyl-

acetaldehyde

acetonitrile

1,3-benzenediamine

morpholine, 4-methyl-

ethanol, 2-methoxy-

hexanedinitrile

ethanol

1,4-benzenediamine

oxirane

uridine, 5-bromo-2-deoxy-

pentanedioic acid

1H-pyrrole-2,5-dione

butanedioic acid, 2,3-dihydroxy-, diethyl ester

2,2-bioxirane

2,5-hexanedione

1,4-dioxane

ethanethioamide

acetamide, N-[5-(aminosulfonyl)-1,3,4-thiadiazol-2-

yl]-

pyrazine

adenosine, 2 ,3-dideoxy-

2-propanone

2-propenoic acid, 2-hydroxyethyl ester

ethane, 1,2-dimethoxy-

acetamide, 2-iodo-

ethanedioic acid, dimethyl ester

1,4-dithiin, 2,3-dihydro-5,6-dimethyl-, 1,1,4,4-

tetraoxide

acetic acid

5-thia-1-azabicyclo[4.2.0]oct-2-ene-2-carboxylic

acid, 3-[[(aminocarbonyl)oxy]methyl]-7-[[2-

furanyl(methoxyimino)acetyl]amino]-8-oxo-

carbamic acid, ethyl ester

benzenesulfonic acid, hydrazide

pentanoic acid, 4-oxo-, methyl ester

ethanamine

propanamide, $\mathrm{N}, \mathrm{N}$-dimethyl-

1H-purin-6-amine

benzenesulfonamide, 4-amino-N-2-pyrimidinyl-

$1 \mathrm{H}$-imidazole

$6 \mathrm{H}$-purine-6-thione, 2-amino-1,7-dihydro-

$2 \mathrm{H}$-1,2,4-benzothiadiazine-7-sulfonamide, 6-chloro-

\begin{tabular}{|c|c|c|c|c|c|}
\hline $\operatorname{cs}(=0)(=0) 0 \operatorname{cccos}(C)(=0)=0$ & -0.52 & -0.68 & -0.16 & -0.90 & -0.38 \\
\hline $\mathrm{CNC}(=\mathrm{O}) \mathrm{NC}$ & -0.49 & -0.62 & -0.13 & -1.27 & -0.78 \\
\hline $\mathrm{O}=\mathrm{C}(\mathrm{O}) \mathrm{CCC}(=\mathrm{O}) \mathrm{C}$ & -0.49 & -0.49 & 0.00 & -0.14 & 0.35 \\
\hline $\mathrm{O}(\mathrm{CcOCCOCCOCCOC} 1) \mathrm{C} 1$ & -0.48 & -1.14 & -0.66 & -0.40 & 0.08 \\
\hline $\mathrm{O}=\mathrm{C}(\mathrm{O}) \mathrm{C}=\mathrm{CC}(=\mathrm{O}) \mathrm{O}$ & -0.48 & 0.05 & 0.53 & 0.21 & 0.69 \\
\hline $\begin{array}{l}\mathrm{O} 4 \mathrm{C}(\mathrm{C}) \mathrm{C}(\mathrm{O}) \mathrm{C}(\mathrm{O}) \mathrm{C}(\mathrm{O}) \mathrm{C} 4 \mathrm{OC} 5 \mathrm{C}(\mathrm{O}) \mathrm{C}( \\
\mathrm{O}) \mathrm{C}(\mathrm{CO}) \mathrm{OC} 5 \mathrm{Oc} 1 \mathrm{cc}(\mathrm{O}) \mathrm{c} 2 \mathrm{C}(=\mathrm{O}) \mathrm{CC}(\mathrm{c} \\
3 \mathrm{ccc}(\mathrm{O}) \mathrm{cc} 3) \mathrm{Oc} 2 \mathrm{c} 1\end{array}$ & -0.44 & -0.52 & -0.08 & -0.24 & 0.20 \\
\hline $\mathrm{O}(\mathrm{COCO} 1) \mathrm{C} 1$ & -0.43 & -0.56 & -0.13 & -0.95 & -0.52 \\
\hline $\mathrm{C}(\# \mathrm{C}) \mathrm{CN}$ & -0.43 & -0.43 & 0.00 & -0.72 & -0.29 \\
\hline $\mathrm{O}=\mathrm{C}(\mathrm{N}) \mathrm{c}(\operatorname{ccc}(\mathrm{N}) \mathrm{c} 1) \mathrm{c} 1$ & -0.41 & -0.18 & 0.23 & 0.02 & 0.43 \\
\hline $\mathrm{O}=\mathrm{C}(\mathrm{OCC} 1 \mathrm{C}) \mathrm{O} 1$ & -0.41 & 0.08 & 0.49 & 0.13 & 0.54 \\
\hline$N(C C N(C 1) C)(C 1) C$ & -0.40 & -0.38 & 0.02 & -0.01 & 0.39 \\
\hline OCC\#C & -0.38 & -0.42 & -0.04 & -0.70 & -0.32 \\
\hline$N(C) C$ & -0.38 & -0.17 & 0.21 & -0.53 & -0.1 \\
\hline $\mathrm{O}=\mathrm{C} 1 \mathrm{CCCN} 1 \mathrm{C}$ & -0.38 & -0.11 & 0.27 & -0.72 & -0.3 \\
\hline $\begin{array}{l}\mathrm{c} 12 \mathrm{ncn}(\mathrm{C} 3 \mathrm{OC}(\mathrm{CO}) \mathrm{C}(\mathrm{O}) \mathrm{C} 3 \mathrm{O}) \mathrm{c} 1 \mathrm{nc}(\mathrm{Cl} \\
) \mathrm{nc} 2 \mathrm{~N}\end{array}$ & -0.37 & -0.73 & -0.36 & -0.28 & 0.0 \\
\hline $\mathrm{O}=\mathrm{C}(\mathrm{N}) \mathrm{c}(\mathrm{cccn} 1) \mathrm{c} 1$ & -0.37 & -0.45 & -0.08 & -0.45 & $-0.0 \varepsilon$ \\
\hline $\mathrm{O}(\mathrm{CCO} 1) \mathrm{C} 1$ & -0.37 & -0.31 & 0.06 & -0.61 & -0.2 \\
\hline $\mathrm{O}(\mathrm{CcOC}) \mathrm{CCOC}$ & -0.36 & -0.48 & -0.12 & 0.12 & $0.4 \varepsilon$ \\
\hline $\mathrm{O}=\mathrm{C}(\mathrm{O}) \mathrm{C}(\mathrm{O})(\mathrm{C}) \mathrm{C}$ & -0.36 & -0.20 & 0.16 & -0.37 & -0.0 \\
\hline $\mathrm{N}(=\mathrm{O})(=\mathrm{O}) \mathrm{C}$ & -0.35 & -0.04 & 0.31 & -0.17 & 0.1 \\
\hline$S=C 1 N C=C N 1 C$ & -0.34 & -0.49 & -0.15 & -0.38 & -0.0 \\
\hline $\mathrm{O}=\mathrm{CC}$ & -0.34 & -0.17 & 0.17 & -0.01 & 0.33 \\
\hline $\mathrm{C}(\# \mathrm{~N}) \mathrm{C}$ & -0.34 & -0.15 & 0.19 & -0.04 & 0.30 \\
\hline $\mathrm{Nc}(\operatorname{cccc} 1 \mathrm{~N}) \mathrm{c} 1$ & -0.33 & -0.39 & -0.06 & 0.01 & 0.34 \\
\hline $\mathrm{O}(\mathrm{CCN}(\mathrm{C} 1) \mathrm{C}) \mathrm{C} 1$ & -0.33 & -0.35 & -0.02 & -0.14 & $0.1 \mathrm{~s}$ \\
\hline $\mathrm{O}(\mathrm{CCO}) \mathrm{CC}$ & -0.32 & -0.42 & -0.10 & -0.28 & 0.04 \\
\hline $\mathrm{C}(\# \mathrm{~N}) \operatorname{Ccccc}(\# \mathrm{~N})$ & -0.32 & 0.35 & 0.67 & -0.14 & 0.18 \\
\hline $\mathrm{OCC}$ & -0.31 & -0.14 & 0.17 & -0.40 & -0.08 \\
\hline $\mathrm{Nc}(\operatorname{ccc}(\mathrm{N}) \mathrm{c} 1) \mathrm{c} 1$ & -0.30 & -0.39 & -0.09 & -0.01 & 0.2 \\
\hline $\mathrm{O}(\mathrm{C} 1) \mathrm{C} 1$ & -0.30 & -0.05 & 0.25 & -0.47 & -0.1 \\
\hline $\begin{array}{l}\mathrm{C} 1=\mathrm{C}(\mathrm{Br}) \mathrm{C}(=\mathrm{O}) \mathrm{NC}(=\mathrm{O}) \mathrm{N} 1 \mathrm{C} 2 \mathrm{OC}(\mathrm{CO} \\
\mathrm{C}(\mathrm{O}) \mathrm{C} 2\end{array}$ & -0.29 & -0.80 & -0.51 & -0.97 & -0.6 \\
\hline $\mathrm{O}=\mathrm{C}(\mathrm{O}) \operatorname{cccc}(=0) \mathrm{O}$ & -0.29 & -0.26 & 0.03 & -0.25 & 0.04 \\
\hline $\mathrm{N} 1 \mathrm{C}(=\mathrm{O}) \mathrm{C}=\mathrm{CC} 1(=\mathrm{O})$ & -0.29 & -0.12 & 0.17 & -0.78 & -0.4 \\
\hline $\mathrm{O}=\mathrm{C}(\mathrm{OCC}) \mathrm{C}(\mathrm{O}) \mathrm{C}(\mathrm{O}) \mathrm{C}(=\mathrm{O}) \mathrm{OCC}$ & -0.29 & -0.08 & 0.21 & -0.57 & -0.2 \\
\hline $\mathrm{C} 1 \mathrm{OC} 1 \mathrm{C} 2 \mathrm{CO} 2$ & -0.28 & -0.58 & -0.30 & -0.36 & -0.0 \\
\hline $\mathrm{O}=\mathrm{C}(\mathrm{CcC}(=\mathrm{O}) \mathrm{C}) \mathrm{C}$ & -0.27 & -0.81 & -0.54 & 0.28 & 0.55 \\
\hline $\mathrm{O}(\mathrm{CCOC} 1) \mathrm{C} 1$ & -0.27 & -0.32 & -0.05 & -0.23 & 0.0 \\
\hline $\mathrm{NC}(=\mathrm{S}) \mathrm{C}$ & -0.26 & -0.83 & -0.57 & -0.30 & -0.0 \\
\hline $\mathrm{CC}(=0) \mathrm{Nc} 1 \mathrm{nnc}(\mathrm{S}(=\mathrm{O})(=\mathrm{O}) \mathrm{N}) \mathrm{s} 1$ & -0.26 & -0.72 & -0.46 & -0.39 & -0.1 \\
\hline$n(\mathrm{ccnc1}) \mathrm{c} 1$ & -0.26 & -0.06 & 0.20 & -0.29 & -0.0 \\
\hline $\mathrm{O} 1 \mathrm{C}(\mathrm{CO}) \mathrm{CCC} 1 \mathrm{~N} 2 \mathrm{c} 3 \mathrm{ncnc}(\mathrm{N}) \mathrm{c} 3 \mathrm{~N}=\mathrm{C} 2$ & -0.25 & -0.65 & -0.40 & -0.53 & -0.2 \\
\hline $\mathrm{O}=\mathrm{C}(\mathrm{C}) \mathrm{C}$ & -0.24 & -0.24 & 0.00 & -0.29 & -0.0 \\
\hline $\mathrm{O}=\mathrm{C}(\mathrm{OCCO}) \mathrm{C}=\mathrm{C}$ & -0.21 & -0.25 & -0.04 & 0.04 & 0.2 \\
\hline $\mathrm{O}(\mathrm{CCOC}) \mathrm{C}$ & -0.21 & -0.21 & 0.00 & 0.03 & 0.2 \\
\hline $\mathrm{O}=\mathrm{C}(\mathrm{N}) \mathrm{Cl}$ & -0.19 & -0.08 & 0.11 & -0.03 & 0.1 \\
\hline $\mathrm{O}=\mathrm{C}(\mathrm{OC}) \mathrm{C}(=\mathrm{O}) \mathrm{OC}$ & -0.17 & -0.58 & -0.41 & 0.01 & 0.1 \\
\hline$C C 1=C(C) S(=0)(=0) \operatorname{CcS} 1(=0)=0$ & -0.17 & 0.04 & 0.21 & -0.93 & -0.7 \\
\hline $\mathrm{O}=\mathrm{C}(\mathrm{O}) \mathrm{C}$ & -0.17 & 0.09 & 0.26 & -0.12 & 0.0 \\
\hline $\begin{array}{l}\mathrm{CON}=\mathrm{C}(\mathrm{C}(=\mathrm{O}) \mathrm{NC} 2 \mathrm{C} 1 \mathrm{SCC}(=\mathrm{C}(\mathrm{N} 1 \mathrm{C} 2 \\
=\mathrm{O}) \mathrm{C}(\mathrm{O})=\mathrm{O}) \operatorname{COC}(\mathrm{N})=\mathrm{O}) \mathrm{c} 3 \mathrm{ccco} 3\end{array}$ & -0.16 & 0.29 & 0.45 & -0.24 & -0.0 \\
\hline $\mathrm{O}=\mathrm{C}(\mathrm{OCC}) \mathrm{N}$ & -0.15 & -0.02 & 0.13 & -0.14 & 0.0 \\
\hline $\mathrm{O}=\mathrm{S}(=\mathrm{O})(\mathrm{NN}) \mathrm{c}(\operatorname{cccc} 1) \mathrm{c} 1$ & -0.14 & 0.01 & 0.15 & 0.26 & 0.4 \\
\hline $\mathrm{CC}(=0) \operatorname{CCC}(=0) O C$ & -0.13 & -0.20 & -0.07 & -0.17 & -0.0 \\
\hline NCC & -0.13 & -0.15 & -0.02 & -0.20 & -0.0 \\
\hline $\mathrm{O}=\mathrm{C}(\mathrm{N}(\mathrm{C}) \mathrm{C}) \mathrm{CC}$ & -0.11 & 0.01 & 0.12 & -0.06 & 0.0 \\
\hline $\mathrm{n}(\mathrm{c}(\mathrm{c}(\mathrm{ncn} 1) \mathrm{c} 1 \mathrm{n} 2) \mathrm{N}) \mathrm{c} 2$ & -0.09 & -0.73 & -0.64 & -0.38 & -0.2 \\
\hline $\mathrm{n} 1 \mathrm{c}(\mathrm{NS}(=\mathrm{O})(=\mathrm{O}) \mathrm{c} 2 \mathrm{ccc}(\mathrm{N}) \mathrm{cc} 2) \mathrm{nccc} 1$ & -0.09 & -0.34 & -0.25 & 0.25 & 0.3 \\
\hline $\mathrm{N} 1 \mathrm{C}=\mathrm{NC}=\mathrm{C} 1$ & -0.08 & 0.06 & 0.14 & -0.21 & -0.1 \\
\hline $\mathrm{N} 1 \mathrm{C}(\mathrm{N})=\mathrm{Nc} 2 \mathrm{ncnc} 2 \mathrm{C} 1=$ & -0.07 & -0.31 & -0.24 & -0.73 & -0.6 \\
\hline $\mathrm{O}=\mathrm{S}(=\mathrm{O})$ & -0.07 & -0.10 & -0.03 & -0.16 & -0.0 \\
\hline
\end{tabular}


3,4-dihydro-, 1,1-dioxide

1H-imidazole, 1-methyl-

propanedioic acid, dimethyl ester

2-pyrrolidinone, 1-ethyl-

2-oxazolidinone, 3-[[(5-nitro-2-

furanyl)methylene]amino]-

2-naphthacenecarboxamide, 4-(dimethylamino)-

1,4,4a,5,5a,6,11,12a-octahydro-3,5,10,12,12apentahydroxy-6-methyl-1,11-dioxo-

1 H-purine-2,6-dione, 3,7-dihydro-1,3-dimethyl-

$1 \mathrm{H}$-imidazole-1-ethanol, 2-methyl-5-nitro-

5-thia-1-azabicyclo[4.2.0]oct-2-ene-2-carboxylic

acid, 3-[[(aminocarbonyl)oxy]methyl]-7-methoxy-8oxo-7-[(2-thienylacetyl)amino]-

2-propenal

methane, dimethoxy-

6H-purine-6-thione, 1,7-dihydro-

formic acid, methyl ester

ethanol, 2-chloro-

2-propen-1-amine

oxirane, methyl-

phenol, 4-amino-

ethanol, 2-(1-methylethoxy)-

thymidine, 3-azido-3-deoxy-

2-propanol

benzenesulfonamide, 4-amino-N-2-thiazolyl-

heptanedinitrile

ethanedinitrile

acetamide, N-(4-aminophenyl)-

isoxazole

hexanedioic acid

benzamide, N-(1-methylethyl)-4-[(2-

methylhydrazino)methyl]-

4H-pyran-4-one, 3-hydroxy-2-methyl-

acetic acid, mercapto-

thiourea, trimethyl-

$1 \mathrm{H}$-purine, 6-[(1-methyl-4-nitro-1 $\mathrm{H}$-imidazol-5yl)thio]-

methane, oxybis-

3-pyridinamine

2-pyridineethanol

1,3-benzenediamine, 4-methyl-

benzenesulfonamide, 4-amino-N-(4-methyl-2-

pyrimidinyl)-

1,2-benzenediamine

benzeneacetamide, 4-[2-hydroxy-3-[(1-

methylethyl)amino]propoxy]-

methanamine, N,N-dimethyl-

propanenitrile

1,3,5-benzenetriol

2-propen-1-ol

acetic acid, methyl ester

ethane, nitro-

propanenitrile, 3-chloro-

urea, tetramethyl-

ethanone, 1-pyrazinyl-

2,5-cyclohexadiene-1,4-dione

phenol, 3-amino-

pyrazine, methyl-

acetic acid, chloro-

adenosine, N-benzoyl-2-deoxy-

2-butenedioic acid, dimethyl ester

hydrazinecarboxamide, 2-[(5-nitro-2-

furanyl)methylene]-

formic acid, ethyl ester

$(\mathrm{N}) \mathrm{c}(\mathrm{c}(\mathrm{cc}(\mathrm{NCNS} 1(=\mathrm{O})=\mathrm{O}) \mathrm{c} 12) \mathrm{Cl}) \mathrm{c} 2$

$\mathrm{N}(\mathrm{C}=\mathrm{CN} 1 \mathrm{C})=\mathrm{C} 1$
$\mathrm{O}=\mathrm{C}(\mathrm{OC}) \mathrm{CC}(=\mathrm{O}) \mathrm{OC}$

$\mathrm{O}=\mathrm{C}(\mathrm{N}(\mathrm{CC} 1) \mathrm{CC}) \mathrm{C} 1$

$\mathrm{O}=\mathrm{C}(\mathrm{OCC} 1) \mathrm{N} 1 \mathrm{~N}=\mathrm{Cc}(\mathrm{Oc}(\mathrm{N}(=\mathrm{O})$

$(=\mathrm{O})) \mathrm{c} 2) \mathrm{c} 2$

$\mathrm{CC} 3 \mathrm{C} 2 \mathrm{C}(\mathrm{O}) \mathrm{C} 1 \mathrm{C}(\mathrm{N}(\mathrm{C}) \mathrm{C}) \mathrm{C}(=\mathrm{C}(\mathrm{C}(\mathrm{N})=$

$\mathrm{O}) \mathrm{C}(=\mathrm{O}) \mathrm{C} 1(\mathrm{O}) \mathrm{C}(=\mathrm{C} 2 \mathrm{C}(=\mathrm{O}) \mathrm{c} 4 \mathrm{C}(\mathrm{O}) \mathrm{cc}$ cc34)O)O

$\mathrm{CN} 1 \mathrm{C}(=\mathrm{O}) \mathrm{N}(\mathrm{C}) \mathrm{c} 2 \mathrm{ncnc} 2 \mathrm{C} 1(=\mathrm{O})$

$\mathrm{Cc} 1 \mathrm{ncc}(\mathrm{N}(=\mathrm{O})=\mathrm{O}) \mathrm{n} 1 \mathrm{CCO}$

$\mathrm{COC} 3(\mathrm{NC}(=\mathrm{O}) \mathrm{Cc} 1 \mathrm{cccs} 1) \mathrm{C} 2 \mathrm{SCC}(=\mathrm{C}$

$-0.06$

$-0.05-0.09$

$-0.04 \quad 0.38$

$-0.04$

1.02

0.67

$-0.04$

0.42

$-0.18$

0.08

1.06

0.13
0.49

$-0.12$

0.13

0.02

$-1.36$

$-1.34$

$-0.72$

0.53

$\mathrm{N} 2 \mathrm{C} 3=\mathrm{O}) \mathrm{C}(\mathrm{O})=\mathrm{O}) \operatorname{COC}(\mathrm{N})=\mathrm{O}$

$\mathrm{O}=\mathrm{CC}=\mathrm{C}$

$\mathrm{O}(\mathrm{COC}) \mathrm{C}$

n1enc2ncnc2c1S

$\mathrm{O}=\mathrm{COC}$

$\mathrm{OCCCl}$

$\mathrm{NCC}=\mathrm{C}$

$\mathrm{O}(\mathrm{C} 1 \mathrm{C}) \mathrm{C} 1$

$\mathrm{Oc}(\operatorname{ccc}(\mathrm{N}) \mathrm{c} 1) \mathrm{c} 1$

$\mathrm{O}(\mathrm{C}(\mathrm{C}) \mathrm{C}) \mathrm{CCO}$

$\mathrm{C} 1=\mathrm{C}(\mathrm{C}) \mathrm{C}(=\mathrm{O}) \mathrm{NC}(=\mathrm{O}) \mathrm{N} 1 \mathrm{C} 2 \mathrm{OC}(\mathrm{CO})$ $\mathrm{C}(\mathrm{N}=\mathrm{N} \# \mathrm{~N}) \mathrm{C} 2$

$\mathrm{OC}(\mathrm{C}) \mathrm{C}$

$\operatorname{c1cc}(\mathrm{N}) \operatorname{ccc} 1 \mathrm{~S}(=\mathrm{O})(=\mathrm{O}) \mathrm{Nc} 2 \mathrm{nccs} 2$

$\mathrm{C}(\# \mathrm{~N}) \operatorname{CCCCCC}(\# \mathrm{~N})$

$\mathrm{C}(\# \mathrm{~N}) \mathrm{C}(\# \mathrm{~N})$

$\mathrm{O}=\mathrm{C}(\mathrm{Nc}(\operatorname{ccc}(\mathrm{N}) \mathrm{c} 1) \mathrm{c} 1) \mathrm{C}$

n1occc1

$\mathrm{O}=\mathrm{C}(\mathrm{O}) \operatorname{ccccc}(=\mathrm{O}) \mathrm{O}$

CNNCc1 $1 \operatorname{ccc}(\operatorname{cc} 1) \mathrm{C}(=\mathrm{O}) \mathrm{NC}(\mathrm{C}) \mathrm{C}$

$-0.02$

$-0.39$

0.00

$-0.37$

-0.37
0.02

$-0.26$

$\begin{array}{ll}-0.02 & 0.22\end{array}$

0.24

0.22

0.24

$\mathrm{O} 1 \mathrm{C}(\mathrm{C})=\mathrm{C}(\mathrm{O}) \mathrm{C}(=\mathrm{O}) \mathrm{C}=\mathrm{C} 1$

$\mathrm{O}=\mathrm{C}(\mathrm{O}) \mathrm{CS}$

$\mathrm{N}(\mathrm{C}(\mathrm{N}(\mathrm{C}) \mathrm{C})=\mathrm{S}) \mathrm{C}$

$\mathrm{N}(=\mathrm{O})$

$(=\mathrm{O}) \mathrm{c} 1 \mathrm{ncn}(\mathrm{C}) \mathrm{c} 1 \mathrm{Sc} 2 \mathrm{ncnc} 3 \mathrm{ncnc} 23$

$\mathrm{O}(\mathrm{C}) \mathrm{C}$

$\mathrm{n}(\operatorname{cccc} 1 \mathrm{~N}) \mathrm{c} 1$

$\mathrm{n}(\mathrm{c}(\mathrm{ccc} 1) \mathrm{CCO}) \mathrm{c} 1$

$\mathrm{Nc}(\mathrm{c}(\operatorname{ccc} 1 \mathrm{~N}) \mathrm{C}) \mathrm{c} 1$

$\mathrm{O}=\mathrm{S}(=\mathrm{O})$

( Nc(nccc1C)n1)c(ccc(N)c2)c2

$\mathrm{Nc}(\mathrm{c}(\mathrm{N}) \mathrm{ccc} 1) \mathrm{c} 1$

$\mathrm{CC}(\mathrm{C}) \mathrm{NCC}(\mathrm{O}) \operatorname{COc} 1 \operatorname{ccc}(\mathrm{CC}(=\mathrm{O})$

$-0.01$

0.19

0.20

0.18

0.19

$-0.19$

$-0.19$

$-0.14$

$-0.17$

$-0.20$

0.11

0.08

0.18

0.37

0.34

0.20

$-0.05$

0.18

$-0.37$

0.04

$-0.31$

0.00

$-0.43$

0.04

0.47

0.43

0.23

$-0.10$

$-0.15$

0.28

0.23

0.04

$-0.01$

$(\mathrm{N})) \mathrm{cc} 1$

$\mathrm{N}(\mathrm{C})(\mathrm{C}) \mathrm{C}$

$\mathrm{C}(\# \mathrm{~N}) \mathrm{CC}$

$\mathrm{Oc}(\mathrm{cc}(\mathrm{O}) \mathrm{cc} 1 \mathrm{O}) \mathrm{c} 1$

$\mathrm{OCC}=\mathrm{C}$

$\mathrm{O}=\mathrm{C}(\mathrm{OC}) \mathrm{C}$

$\mathrm{N}(=\mathrm{O})(=\mathrm{O}) \mathrm{CC}$

$\mathrm{C}(\mathrm{\# N}) \mathrm{CCCl}$

$\mathrm{O}=\mathrm{C}(\mathrm{N}(\mathrm{C}) \mathrm{C}) \mathrm{N}(\mathrm{C}) \mathrm{C}$

$\mathrm{O}=\mathrm{C}(\mathrm{c}(\mathrm{nccn} 1) \mathrm{c} 1) \mathrm{C}$

$\mathrm{O}=\mathrm{C} 1 \mathrm{C}=\mathrm{CC}(=\mathrm{O}) \mathrm{C}=\mathrm{C} 1$

0.72
0.84

0.67

0.79

0.88

$0.29-0.24$

0.07

0.00

0.10

$-0.65$

0.14

0.38

0.21

0.23

0.13

0.15

1.21

$-0.19$

$-0.28$

0.03

$-0.17$

$-0.06$

$-0.24$

-0.24
0.09

0.09

$\begin{array}{lllll}0.10 & -0.09 & -0.19 & 0.84 & 0.74\end{array}$

$-0.33$

0.00

0.10

0.07

$-0.03$

$-0.16$

$-0.26$

$0.11 \quad-0.11$

$-0.22$

0.18

0.07

0.38

0.26

0.02

0.45

0.33

0.07

0.44

$0.15 \quad 0.16$

0.01

$-0.08 \quad-0.23$

Oc $(\operatorname{cccc} 1 \mathrm{~N}) \mathrm{c} 1$

$\mathrm{n}$ (ccnc1C) 1

$\mathrm{O}=\mathrm{C}(\mathrm{O}) \mathrm{CCl}$

$\mathrm{O} 1 \mathrm{C}(\mathrm{CO}) \mathrm{C}(\mathrm{O}) \mathrm{CC} 1 \mathrm{~N} 2 \mathrm{c} 3 \mathrm{ncnc}(\mathrm{NC}(=\mathrm{O})$

$\mathrm{c} 4 \mathrm{ccccc} 4) \mathrm{c} 3 \mathrm{~N}=\mathrm{C} 2$

$\mathrm{O}=\mathrm{C}(\mathrm{OC}) \mathrm{C}=\mathrm{CC}(=\mathrm{O}) \mathrm{OC}$

0.16

$-0.03$

$-0.19$

0.57

0.41

\section{$0.16 \quad 0.04$}

$-0.12$

$-0.14$

$-0.30$

$\begin{array}{ll}0.16 & 0.35 \\ 0.16 & 0.55\end{array}$

0.19

0.39

$-0.01$

$-0.17$

0.21

0.04

0.19

0.27

0.42

$-0.39$

$-0.58$

0.05

0.03

0.28

0.12

0.47

0.25

$-0.03 \quad-0.20$

$\begin{array}{ll}0.18 & 0.00\end{array}$

$0.45 \quad 0.27$

$0.41 \quad 0.23$

$-0.06 \quad-0.25$

$-0.15 \quad-0.35$

$0.21 \quad 0.01$

$0.43 \quad 0.22$
0.19

$\begin{array}{ll}0.19 & -0.02\end{array}$

$\begin{array}{ll}0.18 & -0.04\end{array}$

$\mathrm{O}=\mathrm{C}(\mathrm{N}) \mathrm{NN}=\mathrm{Cc}(\mathrm{Oc}(\mathrm{N}(=\mathrm{O})(=\mathrm{O})) \mathrm{c} 1) \mathrm{c} 1$

0.22

1.21

0.99

0.29

0.07

$\mathrm{O}=\mathrm{COCC}$

0.23

0.32

0.09

$\begin{array}{ll}0.45 & 0.23\end{array}$

$0.23 \quad 0.00$

$0.38 \quad 0.15$ 
methanesulfonamide, N-[4-[1-hydroxy-2-[(1-

methylethyl)amino]ethyl]phenyl]-

gibb-3-ene-1,10-dicarboxylic acid, 2,4a,7-

trihydroxy-1-methyl-8-methylene-, 1,4a-lactone, (1alpha,2,4a-alpha,4b ,10 )-

$1 \mathrm{H}$-imidazole, 2-methyl-

butanoic acid, 3-oxo-, ethyl ester

methane, trimethoxy-

2-propenenitrile

1-propanol

1,2,3-propanetriol, triacetate

4-pyridinamine

2-propanamine

benzamide, N-hydroxy-

3-butyn-2-ol, 2-methyl-

2-furanmethanol

2-butanone

1,2-ethanediamine, N,N,N,N-tetramethyl-

benzenesulfonamide

$1 \mathrm{H}$-imidazole, 1,2-dimethyl-5-nitro-

4-pyridinecarboxylic acid

propanoic acid

benzeneacetic acid, 4-hydroxy-3-methoxy-

acetamide, N,N-diethyl-

I-proline, 1-(3-mercapto-2-methyl-1-oxopropyl)-

2-propenoic acid, 2-hydroxypropyl ester

formaldehyde

butanedioic acid, dimethyl ester

2-propenoic acid

2-propanol, 2-methyl-

pyrimidine, 2-chloro-

3-pyridinecarboxylic acid

2-pyrrolidinone, 1-ethenyl-

2-furanmethanamine

ethyne

2,5-pyrrolidinedione, 3-ethyl-3-methyl-

3H-pyrazol-3-one, 1,2-dihydro-1,5-dimethyl-2-

phenyl-

2-thiazolamine

ethane, 1,1-oxybis[2-ethoxy-]

2,4-pentanedione

guanidine, N-cyano- $\mathrm{N}$-methyl- $\mathrm{N}-[2-[[(5-m e t h y l-1 \mathrm{H}-$ imidazol-4-yl)methyl]thio]ethyl]-

2-propanamine, 2-methyl-

guanidine, N,N,N,N-tetramethyl-

ethanol, 2,2,2-trifluoro-

2-furancarboxaldehyde

ethanone, 1-(3-pyridinyl)-

4-pyridinecarboxaldehyde

propanoic acid, 3-mercapto-

2-pyridinecarboxaldehyde

thiazole

7-oxabicyclo[4.1.0]heptane, 3-oxiranyl-

2-pyridinecarbonitrile

oxirane, (chloromethyl)-

phosphonic acid, bis(1-methylethyl) ester

acetamide, N-(4-hydroxyphenyl)-

pyrrolidine

propanenitrile, 2-methyl-

furan, tetrahydro-

2-propenoic acid, 2-methyl-, 2-hydroxyethyl ester benzenesulfonamide, N-hydroxy-

1-propanamine

ethanamine, $\mathrm{N}$-ethyl-N-nitroso-

2-pyridinamine

thiourea, tetramethyl-

pyrimidine, 2-(1-piperazinyl)-

\begin{tabular}{|c|c|c|c|c|c|}
\hline $\operatorname{c1cc}(\mathrm{S}(=\mathrm{O})(=\mathrm{O}) \mathrm{C}) \operatorname{ccc} 1 \mathrm{C}(\mathrm{O}) \mathrm{CNC}(\mathrm{C}) \mathrm{C}$ & 0.24 & -0.26 & -0.50 & 0.19 & -0.05 \\
\hline $\begin{array}{l}\mathrm{O}=\mathrm{C}(\mathrm{OC}(\mathrm{C} 1 \mathrm{C}(\mathrm{C} 2(\mathrm{CC}(\mathrm{O}) \\
(\mathrm{C} 3=\mathrm{C}) \mathrm{CC} 4) \mathrm{C} 3) \mathrm{C}(=\mathrm{O}) \mathrm{O}) \\
(\mathrm{C} 24) \mathrm{C}=\mathrm{CC} 5 \mathrm{O}) \mathrm{C} 15 \mathrm{C}\end{array}$ & 0.24 & 0.45 & 0.21 & 0.66 & 0.42 \\
\hline $\mathrm{N}(\mathrm{C}=\mathrm{CN} 1)=\mathrm{C} 1 \mathrm{C}$ & 0.24 & 0.61 & 0.37 & 0.36 & 0.12 \\
\hline $\mathrm{O}=\mathrm{C}(\mathrm{OCC}) \mathrm{CC}(=\mathrm{O}) \mathrm{C}$ & 0.25 & -0.20 & -0.45 & 0.19 & -0.06 \\
\hline $\mathrm{O}(\mathrm{C}(\mathrm{OC}) \mathrm{OC}) \mathrm{C}$ & 0.25 & -0.03 & -0.28 & -0.23 & -0.48 \\
\hline$C(\# N) C=C$ & 0.25 & 0.21 & -0.04 & 0.20 & -0.05 \\
\hline OCCC & 0.25 & 0.35 & 0.10 & 0.21 & -0.04 \\
\hline $\mathrm{O}=\mathrm{C}(\mathrm{OCC}(\mathrm{OC}(=\mathrm{O}) \mathrm{C}) \operatorname{coc}(=\mathrm{O}) \mathrm{C}) \mathrm{C}$ & 0.25 & 0.36 & 0.11 & 0.40 & 0.15 \\
\hline$n(\operatorname{ccc}(N) c 1) c 1$ & 0.26 & -0.11 & -0.37 & 0.08 & -0.18 \\
\hline $\mathrm{NC}(\mathrm{C}) \mathrm{C}$ & 0.26 & 0.27 & 0.01 & -0.05 & -0.31 \\
\hline $\mathrm{O}=\mathrm{C}(\mathrm{NO}) \mathrm{c}(\mathrm{cccc} 1) \mathrm{c} 1$ & 0.26 & 0.61 & 0.35 & 0.17 & -0.09 \\
\hline $\mathrm{OC}(\mathrm{CHC})(\mathrm{C}) \mathrm{C}$ & 0.28 & 0.45 & 0.17 & 0.08 & -0.20 \\
\hline $\mathrm{C} 1=\mathrm{C}(\mathrm{CO}) \mathrm{OC}=\mathrm{C} 1$ & 0.28 & 0.45 & 0.17 & 0.25 & -0.03 \\
\hline $\mathrm{O}=\mathrm{C}(\mathrm{CC}) \mathrm{C}$ & 0.29 & 0.26 & -0.03 & 0.41 & 0.12 \\
\hline $\mathrm{N}(\mathrm{CCN}(\mathrm{C}) \mathrm{C})(\mathrm{C}) \mathrm{C}$ & 0.30 & -0.26 & -0.56 & 0.33 & 0.03 \\
\hline $\mathrm{O}=\mathrm{S}(=\mathrm{O})(\mathrm{N}) \mathrm{c}(\operatorname{cccc} 1) \mathrm{c} 1$ & 0.31 & 0.37 & 0.06 & 0.32 & 0.01 \\
\hline $\operatorname{Cc} 1 \mathrm{ncc}(\mathrm{N}(=\mathrm{O})(=0)) n 1 \mathrm{C}$ & 0.31 & 0.97 & 0.66 & -0.01 & -0.32 \\
\hline $\mathrm{O}=\mathrm{C}(\mathrm{O}) \mathrm{c}(\mathrm{ccnc} 1) \mathrm{c} 1$ & 0.32 & 0.69 & 0.37 & 0.28 & -0.04 \\
\hline $\mathrm{O}=\mathrm{C}(\mathrm{O}) \mathrm{CC}$ & 0.33 & 0.58 & 0.25 & 0.31 & -0.02 \\
\hline $\mathrm{O}=\mathrm{C}(\mathrm{O}) \mathrm{Cc}(\operatorname{ccc}(\mathrm{O}) \mathrm{c} 1 \mathrm{OC}) \mathrm{c} 1$ & 0.33 & 0.77 & 0.44 & 1.02 & 0.69 \\
\hline $\mathrm{O}=\mathrm{C}(\mathrm{N}(\mathrm{CC}) \mathrm{CC}) \mathrm{C}$ & 0.34 & 0.50 & 0.16 & 0.50 & 0.16 \\
\hline $\operatorname{CC}(\mathrm{CS}) \mathrm{C}(=\mathrm{O}) \mathrm{N} 1 \mathrm{CCCC} 1 \mathrm{C}(=\mathrm{O})(\mathrm{O})$ & 0.34 & 0.84 & 0.50 & 1.02 & 0.68 \\
\hline $\mathrm{O}=\mathrm{C}(\mathrm{OCC}(\mathrm{O}) \mathrm{C}) \mathrm{C}=\mathrm{C}$ & 0.35 & 0.17 & -0.18 & 0.40 & 0.05 \\
\hline $\mathrm{O}=\mathrm{C}$ & 0.35 & 0.35 & 0.00 & -0.68 & -1.03 \\
\hline $\mathrm{O}=\mathrm{C}(\mathrm{OC}) \mathrm{CCC}(=\mathrm{O}) \mathrm{OC}$ & 0.35 & 0.40 & 0.05 & 0.20 & -0.15 \\
\hline $\mathrm{O}=\mathrm{C}(\mathrm{O}) \mathrm{C}=\mathrm{C}$ & 0.35 & 0.44 & 0.09 & 0.46 & 0.11 \\
\hline $\mathrm{OC}(\mathrm{C})(\mathrm{C}) \mathrm{C}$ & 0.35 & 0.73 & 0.38 & 0.70 & 0.35 \\
\hline $\mathrm{C} 1=\mathrm{CN}=\mathrm{C}(\mathrm{Cl}) \mathrm{N}=\mathrm{C} 1$ & 0.36 & 0.58 & 0.22 & 0.86 & 0.50 \\
\hline $\mathrm{O}=\mathrm{C}(\mathrm{O}) \mathrm{c}(\operatorname{ccc} 1) \mathrm{c} 1$ & 0.36 & 0.69 & 0.33 & 0.29 & -0.07 \\
\hline $\mathrm{O}=\mathrm{C}(\mathrm{N}(\mathrm{C}=\mathrm{C}) \mathrm{CC} 1) \mathrm{C} 1$ & 0.37 & 0.25 & -0.12 & -0.11 & -0.48 \\
\hline $\mathrm{C} 1=\mathrm{C}(\mathrm{CN}) \mathrm{OC}=\mathrm{C} 1$ & 0.37 & 0.44 & 0.07 & -0.43 & -0.80 \\
\hline $\mathrm{C \# C}$ & 0.37 & 0.50 & 0.13 & -0.03 & -0.40 \\
\hline $\operatorname{CCC} 1(\mathrm{C}) \mathrm{CC}(=\mathrm{O}) \mathrm{NC} 1=\mathrm{O}$ & 0.38 & 0.51 & 0.13 & 0.10 & -0.28 \\
\hline $\operatorname{c1} \operatorname{coccc} 1 \mathrm{~N} 2 \mathrm{C}(=\mathrm{O}) \mathrm{C}=\mathrm{C}(\mathrm{C}) \mathrm{N} 2 \mathrm{C}$ & 0.38 & 0.59 & 0.21 & 1.18 & 0.80 \\
\hline s1c $(N)$ ncc1 & 0.38 & 0.83 & 0.45 & 0.33 & -0.05 \\
\hline $\mathrm{O}(\mathrm{CcOCC}) \mathrm{CcOCC}$ & 0.39 & 0.50 & 0.11 & 0.64 & 0.25 \\
\hline $\mathrm{O}=\mathrm{C}(\mathrm{CC}(=\mathrm{O}) \mathrm{C}) \mathrm{C}$ & 0.40 & 0.05 & -0.35 & -0.20 & -0.60 \\
\hline $\mathrm{CNC}(\mathrm{NCCSCC} 1 \mathrm{NC}=\mathrm{NC}=1 \mathrm{C})=\mathrm{NC}(\# \mathrm{~N}$ & 0.40 & 0.57 & 0.17 & 0.42 & 0.02 \\
\hline $\mathrm{NC}(\mathrm{C})(\mathrm{C}) \mathrm{C}$ & 0.40 & 0.72 & 0.32 & 0.81 & 0.41 \\
\hline$N=C(N(C) C) N(C) C$ & 0.41 & -0.28 & -0.69 & -0.23 & -0.64 \\
\hline $\mathrm{FC}(\mathrm{F})(\mathrm{F}) \mathrm{CO}$ & 0.41 & 0.27 & -0.14 & 0.61 & 0.20 \\
\hline $\mathrm{O}=\mathrm{CC}(\mathrm{OC}=\mathrm{C} 1)=\mathrm{C} 1$ & 0.41 & 0.83 & 0.42 & 0.43 & 0.02 \\
\hline $\mathrm{O}=\mathrm{C}(\mathrm{c}(\operatorname{ccc} 1) \mathrm{c} 1) \mathrm{C}$ & 0.43 & 0.49 & 0.06 & 0.45 & 0.02 \\
\hline $\mathrm{O}=\mathrm{Cc}(\operatorname{ccnc} 1) \mathrm{c} 1$ & 0.43 & 0.52 & 0.09 & 0.16 & -0.27 \\
\hline $\mathrm{O}=\mathrm{C}(\mathrm{O}) \mathrm{CCS}$ & 0.43 & 0.52 & 0.09 & 0.34 & -0.09 \\
\hline $\mathrm{O}=\mathrm{Cc}(\mathrm{nccc} 1) \mathrm{c} 1$ & 0.44 & 0.52 & 0.08 & 0.54 & 0.10 \\
\hline s1cncc1 & 0.44 & 0.99 & 0.55 & 0.89 & 0.45 \\
\hline $\mathrm{O}(\mathrm{C} 1 \mathrm{C}(\mathrm{CC}(\mathrm{O} 2) \mathrm{C} 2 \mathrm{C} 3) \mathrm{C} 3) \mathrm{C} 1$ & 0.44 & 1.13 & 0.69 & 0.85 & 0.41 \\
\hline $\mathrm{C}(\# \mathrm{~N}) \mathrm{c}(\mathrm{nccc} 1) \mathrm{c} 1$ & 0.45 & 0.35 & -0.10 & 0.59 & 0.14 \\
\hline $\mathrm{O}(\mathrm{C} 1 \mathrm{CCl}) \mathrm{C} 1$ & 0.45 & 0.63 & 0.18 & 0.35 & -0.10 \\
\hline $\mathrm{O}=\mathrm{P}(\mathrm{OC}(\mathrm{C}) \mathrm{C}) \mathrm{OC}(\mathrm{C}) \mathrm{C}$ & 0.45 & 0.68 & 0.23 & 0.93 & 0.48 \\
\hline $\mathrm{O}=\mathrm{C}(\mathrm{Nc}(\mathrm{ccc}(\mathrm{O}) \mathrm{c} 1) \mathrm{c} 1) \mathrm{C}$ & 0.46 & 0.27 & -0.19 & 0.51 & 0.05 \\
\hline$N(C C C 1) C 1$ & 0.46 & 0.70 & 0.24 & 0.16 & -0.30 \\
\hline $\mathrm{N \# CC}(\mathrm{C}) \mathrm{C}$ & 0.46 & 0.76 & 0.30 & 0.50 & 0.04 \\
\hline $\mathrm{O}(\mathrm{CCC} 1) \mathrm{C} 1$ & 0.46 & 0.94 & 0.48 & 0.35 & -0.11 \\
\hline $\mathrm{O}=\mathrm{C}(\mathrm{OCCO}) \mathrm{C}(=\mathrm{C}) \mathrm{C}$ & 0.47 & 0.30 & -0.17 & 0.27 & -0.20 \\
\hline $\mathrm{O}=\mathrm{S}(=\mathrm{O})(\mathrm{NO}) \mathrm{c}(\mathrm{cccc} 1) \mathrm{c} 1$ & 0.48 & 0.25 & -0.23 & 0.28 & -0.20 \\
\hline $\mathrm{NCCC}$ & 0.48 & 0.34 & -0.14 & 0.31 & -0.17 \\
\hline $\mathrm{O}=\mathrm{NN}(\mathrm{CC}) \mathrm{CC}$ & 0.48 & 0.34 & -0.14 & 0.60 & 0.12 \\
\hline$n(c(N) \operatorname{ccc} 1) c 1$ & 0.48 & 0.53 & 0.05 & 0.42 & -0.06 \\
\hline $\mathrm{N}(\mathrm{C}(\mathrm{N}(\mathrm{C}) \mathrm{C})=\mathrm{S})(\mathrm{C}) \mathrm{C}$ & 0.49 & 0.04 & -0.45 & 0.35 & -0.14 \\
\hline n1ccenc1N2CCNCC2 & 0.49 & 0.35 & -0.14 & 0.32 & -0.17 \\
\hline
\end{tabular}


1-propanol, 3-chloro-

phosphonic acid, (2,2,2-trichloro-1-hydroxyethyl)-, dimethyl ester

ethanone, 1-(2-furanyl)-

benzenesulfonamide, $\mathrm{N}$-(phenylsulfonyl)-

1,4-benzenediamine, 2-nitro-

butanenitrile

ethanone, 1,2-di-2-furanyl-2-hydroxy-

pyrazine, 2,3-dimethyl-

erythro-alpha-galacto-octopyranoside, methyl 6,8-

dideoxy-6-[[[-1-methyl-4-propyl-2-

pyrrolidinyl]carbonyl]amino]-1-thio-

ethanol, 2-(2-butoxyethoxy)-

ethanedioic acid, diethyl ester

2-propanol, 1-methoxy-, acetate

thiourea, N,N-diethyl-

benzamide, 5-(aminosulfonyl)-N-[(1-ethyl-2-

pyrrolidinyl)methyl]-2-methoxy-

ethanamine, $\mathrm{N}$-ethyl-

benzenesulfonamide, N,4-dimethyl-N-nitroso-

propanal

1,4-benzenediol

furo[3',4':6,7]naphtho[2,3-d]-1,3-dioxol-6(5aH)-one,

9-[[4,6-o-ethylidene-beta-glucopyranosyl]oxy]-

5,8,8a,9-tetrahydro-5-(4-hydroxy-3,5-

dimethoxyphenyl)-

1,2-propanediol, 3-(2-methoxyphenoxy)-, 1-

carbamate

heptanedioic acid

2-butanol

benzeneacetic acid, alpha-hydroxy-

phenol, 2-amino-

pentanedioic acid, dimethyl ester

glucose, 2,3,4,5,6-pentaacetate

glucopyranose, pentaacetate

2-naphthalenesulfonic acid

4H-pyran-4-one, 2-ethyl-3-hydroxy-

$2 \mathrm{H}-1,3,2$-oxazaphosphorin-2-amine, N,N-bis(2-

chloroethyl)tetrahydro-, 2-oxide

pyrazine, 2,5-dimethyl-

oxirane, (butoxymethyl)-

2-butanone, oxime

methane, trifluoro-

benzamide

5-thia-1-azabicyclo[4.2.0]oct-2-ene-2-carboxylic

acid, 7-[[aminophenylacetyl]amino]-3-methyl-8-oxo-

2,4,6(1H,3H,5H)-pyrimidinetrione, 5,5-diethyl-

benzenecarboximidamide

pyridine

benzoic acid, 3-amino-

cytidine, N-benzoyl-2-deoxy-

1H-indene-1,3(2H)-dione, 2,2-dihydroxy-

1,3,5-trioxane, 2,4,6-trimethyl-

2-furancarboxaldehyde, 5-methyl-

2-pyridineethanamine, N-methyl-

$2 \mathrm{H}$-pyran, 3,4-dihydro-

benzenesulfonamide, 4-amino-N-(5,6-dimethoxy-4-

pyrimidinyl)-

ethanamine, N,N-dimethyl-

benzoic acid, 3,4,5-trihydroxy-

1,3-propanediol, 2-methyl-2-propyl-, dicarbamate

thiourea, phenyl-
$\mathrm{OCCCCl}$

$\operatorname{COP}(=\mathrm{O})(\mathrm{OC}) \mathrm{C}(\mathrm{O}) \mathrm{C}(\mathrm{Cl})(\mathrm{CI}) \mathrm{Cl}$

$\mathrm{O}=\mathrm{C}(\mathrm{C}(\mathrm{OC}=\mathrm{C} 1)=\mathrm{C} 1) \mathrm{C}$

$\mathrm{O}=\mathrm{S}(=\mathrm{O})(\mathrm{NS}(=\mathrm{O})$

$(=0) c(\operatorname{cccc} 1) c 1) c(\operatorname{cccc} 2) c 2$

$\mathrm{N}(=\mathrm{O})(=\mathrm{O}) \mathrm{c}(\mathrm{c}(\mathrm{N}) \operatorname{ccc} 1 \mathrm{~N}) \mathrm{c} 1$

$\mathrm{C}(\mathrm{\# N}) \mathrm{CCC}$

$\mathrm{O}=\mathrm{C}(\mathrm{C}(\mathrm{OC}=\mathrm{C} 1)=\mathrm{C} 1) \mathrm{C}(\mathrm{O}) \mathrm{C}(\mathrm{OC}=\mathrm{C} 2)$

$=\mathrm{C} 2$

$\mathrm{n}(\mathrm{c}(\mathrm{c}(\mathrm{nc} 1) \mathrm{C}) \mathrm{C}) \mathrm{c} 1$

$\operatorname{CCCC} 1 \mathrm{CC}(\mathrm{N}(\mathrm{C}) \mathrm{C} 1) \mathrm{C}(=\mathrm{O}) \mathrm{NC}(\mathrm{C}(\mathrm{C}) \mathrm{O}$

)C2OC(SC)C(O)C(O)C2O

$\mathrm{O}(\mathrm{CcOccO}) \mathrm{Cccc}$

$\mathrm{O}=\mathrm{C}(\mathrm{OCC}) \mathrm{C}(=\mathrm{O}) \mathrm{OCC}$

$\operatorname{COCC}(\mathrm{OC}(=\mathrm{O}) \mathrm{C}) \mathrm{C}$

$\mathrm{N}(\mathrm{C}(=\mathrm{S}) \mathrm{NCC}) \mathrm{CC}$

$\operatorname{CCN} 1 \mathrm{CCCC} 1 \mathrm{CNC}(=\mathrm{O}) \mathrm{c} 2 \mathrm{cc}(\operatorname{ccc} 2 \mathrm{OC})$ $\mathrm{S}(\mathrm{N})(=\mathrm{O})=\mathrm{O}$

$\mathrm{N}(\mathrm{CC}) \mathrm{CC}$

$\mathrm{O}=\mathrm{S}(=\mathrm{O})(\mathrm{N}(\mathrm{N}=\mathrm{O}) \mathrm{C}) \mathrm{c}(\mathrm{ccc}(\mathrm{c} 1) \mathrm{C}) \mathrm{c} 1$

$\mathrm{O}=\mathrm{CCC}$

$\mathrm{Oc}(\operatorname{ccc}(\mathrm{O}) \mathrm{c} 1) \mathrm{c} 1$

$\mathrm{O} 1 \mathrm{C} 2 \mathrm{COC}(\mathrm{C}) \mathrm{OC} 2 \mathrm{C}(\mathrm{O}) \mathrm{C}(\mathrm{O}) \mathrm{C} 1 \mathrm{OC} 3 \mathrm{C}$

$4 \mathrm{COC}(=0) \mathrm{C} 4 \mathrm{C}(\mathrm{c} 5 \mathrm{cc}(\mathrm{OC}) \mathrm{c}(\mathrm{O}) \mathrm{c}(\mathrm{OC}) \mathrm{c}$

5)c6cc7OCOc7cc36

$\mathrm{O}=\mathrm{C}(\mathrm{OCC}(\mathrm{O}) \operatorname{COc}(\mathrm{c}(\mathrm{OC}) \operatorname{ccc} 1) \mathrm{c} 1) \mathrm{N}$

$\mathrm{O}=\mathrm{C}(\mathrm{O}) \operatorname{cccccc}(=0) \mathrm{O}$

$\mathrm{OC}(\mathrm{CC}) \mathrm{C}$

$\mathrm{O}=\mathrm{C}(\mathrm{O}) \mathrm{C}(\mathrm{O}) \mathrm{c}(\mathrm{cccc} 1) \mathrm{c} 1$

$\mathrm{Oc}(\mathrm{c}(\mathrm{N}) \mathrm{ccc} 1) \mathrm{c} 1$

$\mathrm{O}=\mathrm{C}(\mathrm{OC}) \mathrm{CCCC}(=\mathrm{O}) \mathrm{OC}$

$\mathrm{O}=\mathrm{C}(\mathrm{OCC}(\mathrm{OC}(=\mathrm{O}) \mathrm{C}) \mathrm{C}(\mathrm{OC}(=\mathrm{O}) \mathrm{C}) \mathrm{C}($

$\mathrm{OC}(=\mathrm{O}) \mathrm{C}) \mathrm{C}(\mathrm{OC}(=\mathrm{O}) \mathrm{C}) \mathrm{C}(=\mathrm{O})) \mathrm{C}$

$\mathrm{CC}(=0) \mathrm{OCC} 1 \mathrm{OC}(\mathrm{OC}(=\mathrm{O}) \mathrm{C}) \mathrm{C}(\mathrm{OC}(=$

$\mathrm{O}) \mathrm{C}) \mathrm{C}(\mathrm{OC}(=\mathrm{O}) \mathrm{C}) \mathrm{C} 1 \mathrm{OC}(=\mathrm{O}) \mathrm{C}$

$\mathrm{O}=\mathrm{S}(=\mathrm{O})(\mathrm{O}) \mathrm{c}(\operatorname{ccc}(\mathrm{c} 1 \mathrm{ccc} 2) \mathrm{c} 2) \mathrm{c} 1$

$\mathrm{O} 1 \mathrm{C}(\mathrm{CC})=\mathrm{C}(\mathrm{O}) \mathrm{C}(=\mathrm{O}) \mathrm{C}=\mathrm{C} 1$

$\mathrm{C} 1 \mathrm{CCNP}(=\mathrm{O})(\mathrm{N}(\mathrm{CCCl}) \mathrm{CCCl}) \mathrm{O} 1$

$\mathrm{n}(\mathrm{c}(\mathrm{cnc} 1 \mathrm{C}) \mathrm{C}) \mathrm{c} 1$

$\mathrm{O}(\mathrm{C} 1 \mathrm{COCCCC}) \mathrm{C} 1$

$\mathrm{N}(\mathrm{O})=\mathrm{C}(\mathrm{CC}) \mathrm{C}$

$\mathrm{FC}(\mathrm{F}) \mathrm{F}$

$\mathrm{O}=\mathrm{C}(\mathrm{N}) \mathrm{c}(\operatorname{cccc} 1) \mathrm{c} 1$

$\mathrm{CC} 1=\mathrm{C}(\mathrm{N} 3 \mathrm{C}(\mathrm{SC} 1) \mathrm{C}(\mathrm{NC}(=\mathrm{O}) \mathrm{C}(\mathrm{N}) \mathrm{c} 2 \mathrm{C}$

$\operatorname{cccc} 2) \mathrm{C} 3=\mathrm{O}) \mathrm{C}(\mathrm{O})=\mathrm{O}$

$\operatorname{CCC} 1(\mathrm{CC}) \mathrm{C}(=\mathrm{O}) \mathrm{NC}(=\mathrm{O}) \mathrm{NC} 1(=\mathrm{O})$

$\operatorname{cocccc} 1 \mathrm{C}(=\mathrm{N}) \mathrm{N}$

$\mathrm{n}(\operatorname{cccc} 1) \mathrm{c} 1$

$\mathrm{O}=\mathrm{C}(\mathrm{O}) \mathrm{c}(\operatorname{cccc} 1 \mathrm{~N}) \mathrm{c} 1$

$\mathrm{O} 1 \mathrm{C}(\mathrm{CO}) \mathrm{C}(\mathrm{O}) \mathrm{CC} 1 \mathrm{~N} 2 \mathrm{C}(=\mathrm{O}) \mathrm{N}=\mathrm{C}(\mathrm{NC}($ $=\mathrm{O}) \mathrm{c} 3 \operatorname{ccccc} 3) \mathrm{C}=\mathrm{C} 2$

$\mathrm{O}=\mathrm{C}(\mathrm{c}(\mathrm{c}(\mathrm{C} 1(=\mathrm{O})) \mathrm{ccc} 2) \mathrm{c} 2) \mathrm{C} 1(\mathrm{O}) \mathrm{O} \quad 0.67$

$\mathrm{O}(\mathrm{C}(\mathrm{OC}(\mathrm{O} 1) \mathrm{C}) \mathrm{C}) \mathrm{C} 1 \mathrm{C}$

$\mathrm{O}=\mathrm{CC}(\mathrm{OC}(=\mathrm{C} 1) \mathrm{C})=\mathrm{C} 1$

$\mathrm{n}(\mathrm{c}(\mathrm{ccc} 1) \mathrm{CCNC}) \mathrm{c} 1$

$\mathrm{O}(\mathrm{C}=\mathrm{CCC} 1) \mathrm{C} 1$

$\operatorname{c1cc}(\mathrm{N}) \operatorname{ccc} 1 \mathrm{~S}(=\mathrm{O})$

$(=\mathrm{O}) \mathrm{Nc} 2 \mathrm{c}(\mathrm{OC}) \mathrm{c}(\mathrm{OC}) \mathrm{ncn} 2$

$\mathrm{N}(\mathrm{CC})(\mathrm{C}) \mathrm{C}$

$\mathrm{O}=\mathrm{C}(\mathrm{O}) \mathrm{c}(\mathrm{cc}(\mathrm{O}) \mathrm{c}(\mathrm{O}) \mathrm{c} 1 \mathrm{O}) \mathrm{c} 1$

$\mathrm{O}=\mathrm{C}(\mathrm{OCC}(\mathrm{CCC})(\mathrm{C}) \operatorname{COC}(=\mathrm{O}) \mathrm{N}) \mathrm{N}$

$\mathrm{NC}(=\mathrm{S}) \mathrm{Nc}(\mathrm{cccc} 1) \mathrm{c} 1$

$\begin{array}{ccccc}0.50 & 0.60 & 0.10 & 0.36 & -0.14 \\ 0.51 & -0.28 & -0.79 & 0.81 & 0.30 \\ & & & & \\ 0.52 & 0.80 & 0.28 & 0.73 & 0.21 \\ 0.52 & 1.16 & 0.64 & 1.72 & 1.20 \\ & & & & \\ 0.53 & 0.55 & 0.02 & 1.00 & 0.47 \\ 0.53 & 0.84 & 0.31 & 0.59 & 0.06 \\ 0.54 & 0.33 & -0.21 & 0.80 & 0.26 \\ & & & & \\ 0.54 & 1.03 & 0.49 & 0.63 & 0.09 \\ 0.56 & 0.29 & -0.27 & 0.50 & -0.06 \\ & & & & \\ & & & & \\ 0.56 & 0.29 & -0.27 & 0.63 & 0.07 \\ 0.56 & 0.40 & -0.16 & 1.15 & 0.59 \\ 0.56 & 0.52 & -0.04 & 0.77 & 0.21 \\ 0.57 & 0.60 & 0.03 & 0.62 & 0.05 \\ 0.57 & 0.65 & 0.08 & 1.20 & 0.63 \\ & & & & \\ 0.58 & 0.81 & 0.23 & 0.76 & 0.18 \\ 0.58 & 0.92 & 0.34 & 1.14 & 0.56 \\ 0.59 & 0.33 & -0.26 & 0.31 & -0.28 \\ 0.59 & 1.03 & 0.44 & 0.71 & 0.12 \\ 0.60 & 0.04 & -0.56 & 0.73 & 0.13\end{array}$

\begin{tabular}{|c|c|c|c|c|}
\hline 0.61 & -0.26 & -0.87 & 0.63 & 0.02 \\
\hline 0.61 & 0.72 & 0.11 & 0.51 & -0.10 \\
\hline 0.61 & 0.77 & 0.16 & 0.66 & 0.05 \\
\hline 0.62 & 0.57 & -0.05 & 0.66 & 0.04 \\
\hline 0.62 & 0.60 & -0.02 & 0.35 & -0.27 \\
\hline 0.62 & 0.90 & 0.28 & 0.59 & -0.0 \\
\hline 0.63 & -0.79 & -1.42 & 0.41 & -0.2 \\
\hline 0.63 & -0.75 & -1.38 & 0.53 & -0. \\
\hline 0.63 & 0.01 & -0.62 & -0.38 & -1.0 \\
\hline 0.63 & 0.30 & -0.33 & 0.34 & -0.2 \\
\hline 0.63 & 0.97 & 0.34 & 0.76 & 0.1 \\
\hline 0.63 & 1.03 & 0.40 & 0.62 & -0.01 \\
\hline 0.63 & 1.08 & 0.45 & 1.18 & 0.55 \\
\hline 0.63 & 1.69 & 1.06 & 0.86 & 0.2 \\
\hline 0.64 & 0.58 & -0.06 & 0.97 & 0.33 \\
\hline 0.64 & 0.74 & 0.10 & 0.51 & -0.1 \\
\hline 0.65 & 0.40 & -0.25 & 0.55 & -0 \\
\hline 0.65 & 0.60 & -0.05 & 0.73 & 0.08 \\
\hline 0.65 & 0.75 & 0.10 & 0.28 & -0.37 \\
\hline 0.65 & 0.80 & 0.15 & 0.70 & 0.05 \\
\hline 0.65 & 0.96 & 0.31 & 0.93 & $0.2 \varepsilon$ \\
\hline 0.67 & 0.05 & -0.62 & -0.09 & -0 . \\
\hline 0.67 & 0.32 & -0.35 & 0.84 & 0.1 \\
\hline 0.67 & 0.70 & 0.03 & 0.33 & -0.3 \\
\hline 0.67 & 1.38 & 0.71 & 0.69 & 0.0 \\
\hline 0.68 & 0.48 & -0.20 & 0.59 & $-0.0 s$ \\
\hline 0.69 & 1.21 & 0.52 & 1.40 & 0. \\
\hline 0.70 & -0.24 & -0.94 & 0.72 & 0. \\
\hline 0.70 & 0.53 & -0.17 & 0.71 & 0. \\
\hline 0.70 & 0.86 & 0.16 & 1.17 & 0. \\
\hline 0.70 & 0.98 & 0.28 & 1.06 & \\
\hline & 0. & 0.24 & 0.57 & \\
\hline
\end{tabular}


2-pyridinecarboxylic acid pyrimidine, 5-bromo-

2-butenoic acid

acetic acid ethenyl ester

1-propanamine, 2-methyl-

acetic acid ethyl ester

pyrazine, methoxy-

1,2-benzenedicarboxylic acid

2-butanamine

$1 \mathrm{H}$-pyrrole

1,2-benzenedicarboxylic acid, 3-nitro-

benzeneacetic acid, 4-hydroxy-

ethane, 1,1-difluoro-

1-propanol, 2-methyl-

benzeneacetic acid, alpha-(hydroxymethyl)-

4-pyridinecarboxaldehyde, oxime

butanoic acid

1(3H)-isobenzofuranone

2-propenoic acid, methyl ester

phosphoric acid, triethyl ester

1,3-benzenediol

cyclohexanone

2,3-naphthalenediol, 5-[3-[(1,1-

dimethylethyl)amino]-2-hydroxypropoxy]-1,2,3,4-

tetrahydro-

benzamide, 4-nitro-

ethanol, 2-(phenylamino)-

benzenesulfonamide, 4-methyl-

$1 \mathrm{H}$-indole-2,3-dione

ethanol, 2-butoxy-

3-pyridinecarboxylic acid, methyl ester

urea, phenyl-

ethanone, 1-(4-aminophenyl)-

formic acid, propyl ester

benzoic acid, 4-amino-

2-butanone, 3-methyl-

propanoic acid, methyl ester

cyclohexanone, oxime

benzenesulfonamide, 2-methyl-

piperidine

ethane, 1,1-diethoxy-

ethanone, 1-(2-pyridinyl)-

benzeneacetic acid, 3-hydroxy-

benzenesulfonamide, 2-chloro-5-(2,3-dihydro-1-

hydroxy-3-oxo-1H-isoindol-1-yl)-

1-butanol, 4-chloro-

1,3,5-triazine, hexahydro-1,3,5-trinitro-

2-pyridinecarboxylic acid, ethyl ester

propane, 1-nitro-

4-thia-1-azobicyclo[3.2.0]heptane-2-carboxylic acid,

6-[[amino(4-hydroxyphenyl)acetyl]amino]-3,3-

dimethyl-7-oxo-

1,2-benzenediamine, 4-nitro-

butanal

1-butanol

1,2-benzenediol

benzenesulfonamide, 4-amino-N-(5-methyl-3-

isoxazolyl)-

morphinan-3,6-diol, 7,8-didehydro-4,5-epoxy-17-

methyl- (5-alpha,6-alpha)-

benzenesulfonamide, 4-amino-N-(4,6-dimethyl-2-

pyrimidinyl)-

3-pyrazolidinone, 1-phenyl-

ethane, 1,1-oxybis-

\begin{tabular}{|c|c|c|c|c|c|}
\hline $\mathrm{O}=\mathrm{C}(\mathrm{O}) \mathrm{c}(\mathrm{nccc} 1) \mathrm{c} 1$ & 0.72 & 0.69 & -0.03 & 0.22 & -0.50 \\
\hline $\mathrm{n}(\mathrm{cc}(\mathrm{Br}) \mathrm{cn} 1) \mathrm{c} 1$ & 0.72 & 0.83 & 0.11 & 0.66 & -0.06 \\
\hline $\mathrm{O}=\mathrm{C}(\mathrm{O}) \mathrm{C}=\mathrm{CC}$ & 0.72 & 0.85 & 0.13 & 0.94 & 0.22 \\
\hline $\mathrm{O}=\mathrm{C}(\mathrm{OC}=\mathrm{C}) \mathrm{C}$ & 0.73 & 0.73 & 0.00 & 0.83 & 0.10 \\
\hline $\mathrm{NCC}(\mathrm{C}) \mathrm{C}$ & 0.73 & 0.76 & 0.03 & 0.54 & -0.19 \\
\hline $\mathrm{O}=\mathrm{C}(\mathrm{OCC}) \mathrm{C}$ & 0.73 & 0.86 & 0.13 & 0.74 & 0.01 \\
\hline $\mathrm{O}(\mathrm{c}(\mathrm{nccn} 1) \mathrm{c} 1) \mathrm{C}$ & 0.73 & 0.92 & 0.19 & 0.59 & -0.14 \\
\hline $\mathrm{O}=\mathrm{C}(\mathrm{O}) \mathrm{c}(\mathrm{c}(\mathrm{ccc} 1) \mathrm{C}(=\mathrm{O}) \mathrm{O}) \mathrm{c} 1$ & 0.73 & 1.07 & 0.34 & 1.22 & 0.49 \\
\hline $\mathrm{NC}(\mathrm{CC}) \mathrm{C}$ & 0.74 & 0.76 & 0.02 & 0.79 & 0.05 \\
\hline $\mathrm{C} 1=\mathrm{CC}=\mathrm{CN} 1$ & 0.75 & 0.88 & 0.13 & 0.76 & 0.01 \\
\hline $\begin{array}{l}\mathrm{O}=\mathrm{C}(\mathrm{O}) \mathrm{c}(\mathrm{c}(\mathrm{c}(\mathrm{N}(=\mathrm{O}) \\
(=\mathrm{O})) \mathrm{cc} 1) \mathrm{C}(=\mathrm{O}) \mathrm{O}) \mathrm{c} 1\end{array}$ & 0.75 & 0.89 & 0.14 & 1.11 & 0.36 \\
\hline $\mathrm{O}=\mathrm{C}(\mathrm{O}) \mathrm{Cc}(\operatorname{ccc}(\mathrm{O}) \mathrm{c} 1) \mathrm{c} 1$ & 0.75 & 0.95 & 0.20 & 0.93 & 0.18 \\
\hline $\mathrm{FC}(\mathrm{F}) \mathrm{C}$ & 0.75 & 1.13 & 0.38 & 0.79 & 0.04 \\
\hline $\operatorname{Occ}(\mathrm{C}) \mathrm{C}$ & 0.76 & 0.77 & 0.01 & 0.60 & -0.16 \\
\hline $\mathrm{O}=\mathrm{C}(\mathrm{O}) \mathrm{C}(\mathrm{c}(\operatorname{cccc} 1) \mathrm{c} 1) \mathrm{CO}$ & 0.77 & 0.38 & -0.39 & 0.77 & 0.00 \\
\hline $\mathrm{ON}=\mathrm{Cc} 1 \mathrm{ccn} \operatorname{cc} 1$ & 0.77 & 0.68 & -0.09 & 0.12 & -0.65 \\
\hline $\mathrm{O}=\mathrm{C}(\mathrm{O}) \mathrm{CCC}$ & 0.79 & 1.07 & 0.28 & 0.78 & -0.01 \\
\hline $\mathrm{O}=\mathrm{C}(\mathrm{OCc} 1 \mathrm{cccc} 2) \mathrm{c} 12$ & 0.80 & 0.71 & -0.09 & 0.99 & 0.19 \\
\hline $\mathrm{O}=\mathrm{C}(\mathrm{OC}) \mathrm{C}=\mathrm{C}$ & 0.80 & 0.73 & -0.07 & 0.67 & -0.13 \\
\hline $\mathrm{O}=\mathrm{P}(\mathrm{OCC})(\mathrm{OCC}) \mathrm{OCC}$ & 0.80 & 0.87 & 0.07 & 0.71 & -0.09 \\
\hline Oc $(\operatorname{cccc} 10) c 1$ & 0.80 & 1.03 & 0.23 & 0.70 & -0.10 \\
\hline $\mathrm{O}=\mathrm{C}(\mathrm{CCCC} 1) \mathrm{C} 1$ & 0.81 & 1.13 & 0.32 & 1.03 & 0.22 \\
\hline $\begin{array}{l}\mathrm{CC}(\mathrm{C}) \\
(\mathrm{C}) \mathrm{NCC}(\mathrm{O}) \mathrm{COc} 1 \mathrm{cccc} 2 \mathrm{CC}(\mathrm{O}) \mathrm{C}(\mathrm{O}) \mathrm{Cc} \\
12\end{array}$ & 0.81 & 1.17 & 0.36 & 1.23 & 0.42 \\
\hline $\mathrm{O}=\mathrm{C}(\mathrm{N}) \mathrm{c}(\mathrm{ccc}(\mathrm{N}(=\mathrm{O})(=\mathrm{O})) \mathrm{c} 1) \mathrm{c} 1$ & 0.82 & 0.56 & -0.26 & 0.31 & -0.51 \\
\hline $\mathrm{OCCNc}(\operatorname{cccc} 1) \mathrm{c} 1$ & 0.82 & 0.65 & -0.17 & 0.73 & -0.09 \\
\hline $\mathrm{O}=\mathrm{S}(=\mathrm{O})(\mathrm{N}) \mathrm{c}(\operatorname{ccc}(\mathrm{c} 1) \mathrm{C}) \mathrm{c} 1$ & 0.82 & 0.92 & 0.10 & 0.72 & -0.10 \\
\hline $\mathrm{O}=\mathrm{C}(\mathrm{Nc}(\mathrm{c} 1 \mathrm{ccc} 2) \mathrm{c} 2) \mathrm{C} 1(=\mathrm{O})$ & 0.83 & 0.45 & -0.38 & 0.89 & 0.06 \\
\hline $\mathrm{O}(\mathrm{CCCC}) \mathrm{CCO}$ & 0.83 & 0.57 & -0.26 & 0.78 & -0.05 \\
\hline $\mathrm{O}=\mathrm{C}(\mathrm{OC}) \mathrm{c}(\mathrm{cccn} 1) \mathrm{c} 1$ & 0.83 & 0.64 & -0.19 & 0.45 & -0.38 \\
\hline $\mathrm{O}=\mathrm{C}(\mathrm{Nc}(\operatorname{cccc} 1) \mathrm{c} 1) \mathrm{N}$ & 0.83 & 0.71 & -0.12 & 0.85 & 0.02 \\
\hline $\mathrm{O}=\mathrm{C}(\mathrm{c}(\operatorname{ccc}(\mathrm{N}) \mathrm{c} 1) \mathrm{c} 1) \mathrm{C}$ & 0.83 & 0.76 & -0.07 & 1.31 & 0.48 \\
\hline $\mathrm{O}=\mathrm{COCCC}$ & 0.83 & 0.81 & -0.02 & 0.93 & 0.10 \\
\hline $\mathrm{O}=\mathrm{C}(\mathrm{O}) \mathrm{c}(\operatorname{ccc}(\mathrm{N}) \mathrm{c} 1) \mathrm{c} 1$ & 0.83 & 0.96 & 0.13 & 0.78 & -0.05 \\
\hline $\mathrm{O}=\mathrm{C}(\mathrm{C}(\mathrm{C}) \mathrm{C}) \mathrm{C}$ & 0.84 & 0.67 & -0.17 & 0.78 & -0.06 \\
\hline $\mathrm{O}=\mathrm{C}(\mathrm{OC}) \mathrm{CC}$ & 0.84 & 0.86 & 0.02 & 0.68 & -0.16 \\
\hline $\mathrm{N}(\mathrm{O})=\mathrm{C}(\mathrm{CCCC} 1) \mathrm{C} 1$ & 0.84 & 0.91 & 0.07 & 1.52 & 0.68 \\
\hline $\mathrm{O}=\mathrm{S}(=\mathrm{O})(\mathrm{N}) \mathrm{c}(\mathrm{c}(\mathrm{ccc} 1) \mathrm{C}) \mathrm{c} 1$ & 0.84 & 0.92 & 0.08 & 0.61 & -0.23 \\
\hline $\mathrm{N}(\mathrm{CCCC} 1) \mathrm{C} 1$ & 0.84 & 1.19 & 0.35 & 0.97 & 0.13 \\
\hline $\mathrm{O}(\mathrm{C}(\mathrm{OCC}) \mathrm{C}) \mathrm{CC}$ & 0.84 & 1.20 & 0.36 & 1.19 & 0.35 \\
\hline $\mathrm{O}=\mathrm{C}(\mathrm{c}(\mathrm{nccc} 1) \mathrm{c} 1) \mathrm{C}$ & 0.85 & 0.49 & -0.36 & 0.69 & -0.16 \\
\hline $\mathrm{OC}(=\mathrm{O}) \mathrm{Cc} 1 \mathrm{cc}(\mathrm{O}) \mathrm{ccc} 1$ & 0.85 & 0.95 & 0.10 & 0.93 & 0.08 \\
\hline $\begin{array}{l}\mathrm{NS}(=\mathrm{O}) \\
(=\mathrm{O}) \mathrm{c} 1 \mathrm{cc}(\operatorname{ccc} 1 \mathrm{Cl}) \mathrm{C} 2(\mathrm{O}) \mathrm{NC}(=\mathrm{O}) \mathrm{c} 3 \mathrm{ccc} \\
\mathrm{cc} 23\end{array}$ & 0.85 & 1.01 & 0.16 & 1.27 & 0.42 \\
\hline OCCCCCI & 0.85 & 1.10 & 0.25 & 0.97 & 0.12 \\
\hline $\begin{array}{l}\mathrm{N}(=\mathrm{O})(=\mathrm{O}) \mathrm{N}(\mathrm{CN}(\mathrm{N}(=\mathrm{O}) \\
(=\mathrm{O}) \mathrm{CN} 1 \mathrm{~N}(=\mathrm{O})(=\mathrm{O})) \mathrm{C} 1\end{array}$ & 0.87 & 0.68 & -0.19 & 1.07 & 0.20 \\
\hline $\mathrm{O}=\mathrm{C}(\mathrm{OCC}) \mathrm{c}(\mathrm{nccc} 1) \mathrm{c} 1$ & 0.87 & 0.82 & -0.05 & 1.32 & 0.45 \\
\hline $\mathrm{N}(=\mathrm{O})(=\mathrm{O}) \mathrm{CCC}$ & 0.87 & 0.95 & 0.08 & 0.91 & 0.04 \\
\hline $\begin{array}{l}\operatorname{c1cc}(\mathrm{O}) \operatorname{ccc} 1 \mathrm{C}(\mathrm{N}) \mathrm{C}(=\mathrm{O}) \mathrm{NC} 2 \mathrm{C}(=\mathrm{O}) \mathrm{N} 3 \\
\mathrm{C}(\mathrm{C}(=\mathrm{O}) \mathrm{O}) \mathrm{C}(\mathrm{C})(\mathrm{C}) \mathrm{SC} 23\end{array}$ & 0.87 & 0.97 & 0.10 & 0.75 & -0.12 \\
\hline $\mathrm{N}(=\mathrm{O})(=\mathrm{O}) \mathrm{c}(\operatorname{ccc}(\mathrm{N}) \mathrm{c} 1 \mathrm{~N}) \mathrm{c} 1$ & 0.88 & 0.55 & -0.33 & 0.84 & -0.04 \\
\hline $\mathrm{O}=\mathrm{CCCC}$ & 0.88 & 0.82 & -0.06 & 1.10 & 0.22 \\
\hline OCCCC & 0.88 & 0.84 & -0.04 & 0.84 & -0.04 \\
\hline $\mathrm{Oc}(\mathrm{c}(\mathrm{O}) \mathrm{ccc} 1) \mathrm{c} 1$ & 0.88 & 1.03 & 0.15 & 0.74 & -0.14 \\
\hline $\mathrm{Cc} 1 \mathrm{cc}(\mathrm{NS}(=\mathrm{O})(=\mathrm{O}) \mathrm{c} 2 \operatorname{ccc}(\mathrm{N}) \mathrm{cc} 2) \mathrm{no} 1$ & 0.89 & 0.48 & -0.41 & 0.79 & -0.10 \\
\hline $\begin{array}{l}\mathrm{C} 1=\mathrm{CC} 2 \mathrm{C}(\mathrm{N}(\mathrm{C}) \mathrm{C} 5) \mathrm{Cc} 3 \mathrm{ccc}(\mathrm{O}) \mathrm{c} 4 \mathrm{c} 3 \mathrm{C} \\
2(\mathrm{C} 5) \mathrm{C}(\mathrm{O} 4) \mathrm{C} 1 \mathrm{O}\end{array}$ & 0.89 & 0.72 & -0.17 & 0.99 & 0.10 \\
\hline $\begin{array}{l}\mathrm{O}=\mathrm{S}(=\mathrm{O}) \\
(\mathrm{Nc}(\mathrm{nc}(\mathrm{cc} 1 \mathrm{C}) \mathrm{C}) \mathrm{n} 1) \mathrm{c}(\mathrm{ccc}(\mathrm{N}) \mathrm{c} 2) \mathrm{c} 2\end{array}$ & 0.89 & 0.76 & -0.13 & 0.43 & -0.46 \\
\hline $\mathrm{O}=\mathrm{C}(\mathrm{NN}(\mathrm{c}(\operatorname{cccc} 1) \mathrm{c} 1) \mathrm{C} 2) \mathrm{C} 2$ & 0.89 & 0.77 & -0.12 & 0.46 & -0.43 \\
\hline $\mathrm{O}(\mathrm{CC}) \mathrm{CC}$ & 0.89 & 1.05 & 0.16 & 1.12 & 0.23 \\
\hline
\end{tabular}


2-butanol, 2-methyl-

benzenamine, 4-methylbenzenesulfonate

1,2-benzisothiazol-3(2H)-one, 1,1-dioxide

$4,6(1 \mathrm{H}, 5 \mathrm{H})$-pyrimidinedione, 5-ethyldihydro-5phenyl-

2,4-pyrimidinediamine, 5-[(3,4,5-

trimethoxyphenyl)methyl]-

2-pentanone

methane, chloro-

1,4-benzenediol, 2-methyl-

acetic acid, dichloro-

ethanone, 1-(1H-pyrrol-2-yl)-

propane, 2-nitro-

2-propenoic acid, 2-methyl-

propanoic acid, 2-methyl-

1-propyne

acetic acid, chloro-, ethyl ester

methane, isothiocyanato-

propane, 2-methoxy-2-methyl-

1,3-dioxolo[4,5-g]quinoline-7-carboxylic acid, 5-

ethyl-5,8-dihydro-8-oxo-

benzenamine, 4-methoxy-

2H-pyran, tetrahydro-

pyrazine, trimethyl-

2-propenoic acid, 1,2-ethanediylbis(oxy-2,1-

ethanediyl) ester

propanedioic acid, diethyl ester

2-propenoic acid, 2-methyl-, 2-hydroxypropyl ester

benzenamine, 4,4-sulfonylbis-

\section{1-butanamine}

benzenepropanenitrile, -oxo-

benzaldehyde, 3-hydroxy-4-methoxy-

benzeneacetic acid, alpha-(hydroxymethyl)-, (1alpha,2-beta,4-beta,5-alpha,7-beta)-9-methyl-3oxa-9-azatricyclo[3.3.1.02,4]non-7-yl ester, (alpha)-

2,4,7-pteridinetriamine, 6-phenylurea, N,N-dimethyl-N-phenyl-

strychnidin-10-one, 2,3-dimethoxy-

3-pentanone

1,1-ethanediol, 2,2,2-trichloro-

1,2-benzenedicarbonitrile

2-furancarboxylic acid, methyl ester

alpha-glucofuranose, 1,2-o-(2,2,2-

trichloroethylidene)-

2-thiophenecarboxaldehyde

hexanedioic acid, dimethyl ester

acetamide, 2,2,2-trichloro-

ethene, ethoxy-

benzoic acid, 4-hydroxy-3,5-dimethoxy-

1,3-benzodioxole-5-carboxaldehyde

1,3-propanediol, 2,2-bis(bromomethyl)-

pyrazine, 2-ethyl-3-methyl-

methane, chlorodifluoro-

benzenemethanamine

ethanol, 2,2-[(4-methylphenyl)imino]bis-

benzenemethanol

benzenemethanol, 4-methoxy-

piperazine, 1-phenyl-

propanenitrile, 2,2-azobis[2-methyl-

pyridine, 2-methyl-

2-propen-1-amine, N-2-propenyl-

3,5-heptanedione

acetic acid, bromo-, ethyl ester

\begin{tabular}{|c|c|c|c|c|c|}
\hline $\mathrm{OC}(\mathrm{CC})(\mathrm{C}) \mathrm{C}$ & 0.89 & 1.22 & 0.33 & 1.19 & 0.30 \\
\hline $\operatorname{c} 2 \operatorname{ccc}(\operatorname{cc} 2) \mathrm{N}$ & 0.90 & 1.08 & 0.18 & 0.89 & -0.01 \\
\hline $\mathrm{O}=\mathrm{C}(\mathrm{NS}(=\mathrm{O})(=\mathrm{O}) \mathrm{c} 1 \mathrm{ccc} 2) \mathrm{c} 12$ & 0.91 & 0.45 & -0.46 & 0.09 & -0.82 \\
\hline $\operatorname{CCC} 1(\mathrm{C}(=\mathrm{O}) \mathrm{NCNC} 1(=\mathrm{O})) \operatorname{c} 2 \operatorname{ccccc} 2$ & 0.91 & 0.73 & -0.18 & 0.62 & -0.2 \\
\hline $\begin{array}{l}\mathrm{COc} 2 \mathrm{cc}(\mathrm{Cc} 1 \mathrm{cnc}(\mathrm{N}) \mathrm{nc} 1 \mathrm{~N}) \mathrm{cc}(\mathrm{OC}) \mathrm{c} 2 \mathrm{O} \\
\mathrm{C}\end{array}$ & 0.91 & 0.73 & -0.18 & 1.26 & 0.35 \\
\hline $\mathrm{O}=\mathrm{C}(\mathrm{CCC}) \mathrm{C}$ & 0.91 & 0.75 & -0.16 & 0.87 & -0.0 \\
\hline $\mathrm{CIC}$ & 0.91 & 1.09 & 0.18 & 0.67 & -0.2 \\
\hline $\mathrm{Oc}(\mathrm{c}(\mathrm{cc}(\mathrm{O}) \mathrm{c} 1) \mathrm{C}) \mathrm{c} 1$ & 0.91 & 1.58 & 0.67 & 0.98 & 0.07 \\
\hline $\mathrm{O}=\mathrm{C}(\mathrm{O}) \mathrm{C}(\mathrm{Cl}) \mathrm{Cl}$ & 0.92 & 0.52 & -0.40 & 0.99 & 0.07 \\
\hline $\mathrm{O}=\mathrm{C}(\mathrm{C}(\mathrm{NC}=\mathrm{C} 1)=\mathrm{C} 1) \mathrm{C}$ & 0.93 & 0.56 & -0.37 & 0.82 & -0 \\
\hline $\mathrm{N}(=\mathrm{O})(=\mathrm{O}) \mathrm{C}(\mathrm{C}) \mathrm{C}$ & 0.93 & 0.87 & -0.06 & 0.71 & -0.2 \\
\hline $\mathrm{O}=\mathrm{C}(\mathrm{O}) \mathrm{C}(=\mathrm{C}) \mathrm{C}$ & 0.93 & 0.99 & 0.06 & 0.63 & \\
\hline $\mathrm{O}=\mathrm{C}(\mathrm{O}) \mathrm{C}(\mathrm{C}) \mathrm{C}$ & 0.94 & 1.00 & 0.06 & 0.78 & -0.1 \\
\hline$C(\# C) C^{\prime}$ & 0.94 & 1.04 & 0.10 & 0.92 & -0.0 \\
\hline $\mathrm{O}=\mathrm{C}(\mathrm{OCC}) \mathrm{CCl}$ & 0.94 & 1.12 & 0.18 & 1.14 & 0.2 \\
\hline$N(=C=S) C^{\prime}$ & 0.94 & 1.30 & 0.36 & 1.07 & 0.1 \\
\hline $\mathrm{O}(\mathrm{C}(\mathrm{C})(\mathrm{C}) \mathrm{C}) \mathrm{C}$ & 0.94 & 1.43 & 0.49 & 1.53 & 0.5 \\
\hline $\begin{array}{l}\mathrm{C} 1 \mathrm{Oc} 2 \operatorname{cc} 3 \mathrm{~N}(\mathrm{CC}) \mathrm{C}=\mathrm{C}(\mathrm{C}(=\mathrm{O}) \mathrm{O}) \mathrm{C}(=\mathrm{O}) \\
\mathrm{c} 3 \mathrm{cc} 2 \mathrm{O} 1\end{array}$ & 0.94 & 1.70 & 0.76 & 0.86 & \\
\hline $\mathrm{O}(\mathrm{c}(\mathrm{ccc}(\mathrm{N}) \mathrm{c} 1) \mathrm{c} 1) \mathrm{C}$ & 0.95 & 1.16 & 0.21 & 1.01 & 0.0 \\
\hline $\mathrm{O}(\mathrm{CCCC} 1) \mathrm{C} 1$ & 0.95 & 1.43 & 0.48 & 1.16 & 0.2 \\
\hline $\mathrm{n}(\mathrm{c}(\mathrm{c}(\mathrm{nc} 1 \mathrm{C}) \mathrm{C}) \mathrm{C}) \mathrm{c} 1$ & 0.95 & 1.58 & 0.63 & 1.11 & 0.1 \\
\hline $\begin{array}{l}\mathrm{O}=\mathrm{C}(\mathrm{Occoc} \operatorname{coc} \operatorname{coc}(=\mathrm{O}) \mathrm{C}=\mathrm{C}) \mathrm{C}= \\
\mathrm{C}\end{array}$ & 0.96 & 0.57 & -0.39 & 1.29 & 0.3 \\
\hline $\mathrm{O}=\mathrm{C}(\mathrm{OCC}) \mathrm{CC}(=\mathrm{O}) \mathrm{OCC}$ & 0.96 & 0.90 & -0.06 & 0.93 & -0.0 \\
\hline $\mathrm{O}=\mathrm{C}(\mathrm{OCC}(\mathrm{O}) \mathrm{C}) \mathrm{C}(=\mathrm{C}) \mathrm{C}$ & 0.97 & 0.72 & -0.25 & 0.73 & -0.2 \\
\hline $\begin{array}{l}\mathrm{O}=\mathrm{S}(=\mathrm{O}) \\
(\mathrm{c}(\operatorname{ccc}(\mathrm{N}) \mathrm{c} 1) \mathrm{c} 1) \mathrm{c}(\operatorname{ccc}(\mathrm{N}) \mathrm{c} 2) \mathrm{c} 2\end{array}$ & 0.97 & 0.77 & -0.20 & 1.19 & 0.2 \\
\hline NCCCC & 0.97 & 0.83 & -0.14 & 0.85 & -0.1 \\
\hline $\mathrm{O}=\mathrm{C}(\mathrm{c}(\operatorname{cccc} 1) \mathrm{c} 1) \mathrm{CC}(\# \mathrm{~N})$ & 0.97 & 0.83 & -0.14 & 1.32 & 0.3 \\
\hline $\mathrm{O}=\mathrm{Cc}(\operatorname{ccc}(\mathrm{OC}) \mathrm{c} 10) \mathrm{c} 1$ & 0.97 & 1.05 & 0.08 & 1.36 & 0.3 \\
\hline $\begin{array}{l}\mathrm{CN} 1 \mathrm{C} 2 \mathrm{CC}(\mathrm{CC} 1 \mathrm{C} 3 \mathrm{OC} 23) \mathrm{OC}(=\mathrm{O}) \mathrm{C}(\mathrm{C} \\
\mathrm{O}) \mathrm{c} 4 \mathrm{ccccc} 4\end{array}$ & 0.98 & 0.39 & -0.59 & 1.40 & 0.4 \\
\hline
\end{tabular}

$\mathrm{Nc3nc}(\mathrm{N}) \mathrm{c} 2 \mathrm{nc}(\mathrm{c} 1 \mathrm{ccccc} 1) \mathrm{c}(\mathrm{N}) \mathrm{nc} 2 \mathrm{n} 3$

$\mathrm{O}=\mathrm{C}(\mathrm{N}(\mathrm{C}) \mathrm{C}) \mathrm{Nc}(\operatorname{cccc} 1) \mathrm{c} 1$

0.98

0.98

0.80

$-0.18$

1.21

0.23

$\mathrm{O}=\mathrm{C}(\mathrm{N}(\mathrm{c}(\mathrm{c}) \mathrm{C} 1(\mathrm{C}(\mathrm{N}(\mathrm{C} 2) \mathrm{CC}(\mathrm{C} 3 \mathrm{C} 4 \mathrm{C} 5$

$\mathrm{OC} 6)=\mathrm{C} 6) \mathrm{C} 3) \mathrm{C} 2) \mathrm{cc}(\mathrm{OC}) \mathrm{c} 7 \mathrm{OC}) \mathrm{c} 7) \mathrm{C} 1$

4)C5

$\mathrm{O}=\mathrm{C}(\mathrm{CC}) \mathrm{CC}$

$\mathrm{OC}(\mathrm{O}) \mathrm{C}(\mathrm{Cl})(\mathrm{Cl}) \mathrm{Cl}$

$\mathrm{C}(\# \mathrm{~N}) \mathrm{c}(\mathrm{c}(\mathrm{C}(\# \mathrm{~N})) \mathrm{ccc} 1) \mathrm{c} 1$

$\mathrm{O}=\mathrm{C}(\mathrm{OC}) \mathrm{C}(\mathrm{OC}=\mathrm{C} 1)=\mathrm{C} 1$

$\mathrm{O}(\mathrm{C}(\mathrm{OC}(\mathrm{C} 1 \mathrm{O}) \mathrm{C}(\mathrm{O}) \mathrm{CO}) \mathrm{C} 1 \mathrm{O} 2) \mathrm{C} 2 \mathrm{C}(\mathrm{Cl}$

)$(\mathrm{Cl}) \mathrm{Cl}$

$\mathrm{O}=\mathrm{CC}(\mathrm{SC}=\mathrm{C} 1)=\mathrm{C} 1$

$\mathrm{O}=\mathrm{C}(\mathrm{OC}) \operatorname{CCCCC}(=\mathrm{O}) \mathrm{OC}$

$\mathrm{O}=\mathrm{C}(\mathrm{N}) \mathrm{C}(\mathrm{Cl})(\mathrm{Cl}) \mathrm{Cl}$

$\mathrm{O}(\mathrm{C}=\mathrm{C}) \mathrm{CC}$

$\mathrm{O}=\mathrm{C}(\mathrm{O}) \mathrm{c}(\mathrm{cc}(\mathrm{OC}) \mathrm{c}(\mathrm{O}) \mathrm{c} 1 \mathrm{OC}) \mathrm{c} 1$

$\mathrm{O}=\mathrm{Cc}(\operatorname{ccc}(\mathrm{OCO} 1) \mathrm{c} 12) \mathrm{c} 2$

$\mathrm{OCC}(\mathrm{CBr})(\mathrm{CBr}) \mathrm{CO}$

$\mathrm{n}(\mathrm{c}(\mathrm{c}(\mathrm{nc} 1) \mathrm{CC}) \mathrm{C}) \mathrm{c} 1$

$\mathrm{FC}(\mathrm{F}) \mathrm{Cl}$

$\mathrm{NCC}(\operatorname{cccc} 1) \mathrm{c} 1$

$\operatorname{OCCN}(\mathrm{c}(\operatorname{ccc}(\mathrm{c} 1) \mathrm{C}) \mathrm{c} 1) \mathrm{CCO}$

$\mathrm{OCc}(\operatorname{cccc} 1) \mathrm{c} 1$

$\mathrm{O}(\mathrm{c}(\operatorname{ccc}(\mathrm{c} 1) \mathrm{CO}) \mathrm{c} 1) \mathrm{C}$

$\mathrm{N}$ (c(cccc1)c1)(CCNC2)C2

$\mathrm{C}(\# \mathrm{~N}) \mathrm{C}(\mathrm{N}=\mathrm{NC}(\mathrm{C}(\# \mathrm{~N}))(\mathrm{C}) \mathrm{C})(\mathrm{C}) \mathrm{C}$

$\mathrm{n}(\mathrm{c}(\mathrm{ccc} 1) \mathrm{C}) \mathrm{c} 1$

$\mathrm{N}(\mathrm{CC}=\mathrm{C}) \mathrm{CC}=\mathrm{C}$

$\mathrm{O}=\mathrm{C}(\mathrm{CC}(=\mathrm{O}) \mathrm{CC}) \mathrm{CC}$

$\mathrm{O}=\mathrm{C}(\mathrm{OCC}) \mathrm{CBr}$

$\begin{array}{lllll}0.98 & 1.49 & 0.51 & 1.85 & 0.87\end{array}$

0.99

0.99

0.99

1.00

1.02

1.02

1.03

1.04

1.04

1.04

1.05

1.06

1.07

1.08

1.09

1.09

1.10

1.10

1.10

1.10

1.11

1.11

1.12

1.12
0.75

0.98

1.09

0.95

0.68

1.53

1.39

0.83

0.91

1.04

1.77

0.85

1.53

0.89

1.07

1.18

1.08

1.16

1.54

2.87

1.35

1.52

1.03

1.21
$-0.24$

$-0.01$

0.10

$-0.05$

$-0.34$

0.51

0.36

$-0.21$

$-0.13$

0.00

0.72

$-0.21$

0.46

$-0.19$

$-0.02$

0.09

$-0.02$

0.06

0.44

1.77

0.24

0.41

$-0.09$

0.09
$0.88-0.11$

\section{$\begin{array}{ll}1.19 & 0.20\end{array}$}

$0.98 \quad-0.01$

$1.21 \quad 0.21$

$0.19-0.83$

$1.00-0.02$

$0.94 \quad-0.09$

$0.98 \quad-0.06$

$1.19 \quad 0.15$

$1.55 \quad 0.51$

$0.79-0.26$

$1.03-0.03$

$1.20 \quad 0.13$

$0.90-0.19$

$0.85-0.24$

$1.07-0.03$

$1.28 \quad 0.18$

$\begin{array}{ll}1.54 & 0.44\end{array}$

$2.43 \quad 1.33$

$\begin{array}{ll}1.25 & 0.14\end{array}$

$0.87-0.24$

$\begin{array}{ll}1.18 & 0.06\end{array}$

$1.20 \quad 0.08$
$0.98 \quad-0.10$ 
benzenemethanol, 2-methoxy-

ethene

acetamide, 2,2-dichloro-N-[2-hydroxy-1-

(hydroxymethyl)-2-(4-nitrophenyl)ethyl]-

sulfuric acid, diethyl ester

benzenesulfonamide, 4-chloro-

2-propenoic acid, 3-(3,4-dihydroxyphenyl)-

formamide, N-phenyl-

phenol, 2,6-dimethoxy-

2-propenal, 3-(2-furanyl)-

$1 \mathrm{H}$-isoindole-1,3(2H)-dione

ethanol, 2-[2-(benzoyloxy)ethoxy]-

2(3H)-benzoxazolone

acetamide, N-phenyl-

ethanol, 2-phenoxy-

1,2-ethanediol, dinitrate

1-butanol, 3-methyl-

butanoic acid, 3-methyl-

benzenepropanoic acid, 4-hydroxy-

pyridine, 3-(1-methyl-2-pyrrolidinyl)-

pyrazine, (methylthio)-

benzenamine, 2-methoxy-

methane, tetrafluoro-

butanoic acid, 2-methyl-

benzoic acid, 2-(acetyloxy)-

methane, bromo-

pentanol

morphinan-6-ol, 7,8-didehydro-4,5-epoxy-3-

methoxy-17-methyl-, (5-alpha,6-alpha)-

$2 \mathrm{H}-1,2,4$-benzothiadiazine, 7-chloro-3-methyl-, 1,1-

2-butanone, 3,3-dimethyl-

pyridine, 3-methyl-

butane, 2-nitro-

butanedioic acid, diethyl ester

ethane, 1,1,1 -[methylidynetris(oxy)]tris-

benzaldehyde, 4-hydroxy-3-methoxy-

carbonic acid, diethyl ester

3-pentanol

benzoic acid, 2-amino-

propanoic acid, ethyl ester

1H-pyrrole, 1-methyl-

pyridine, 4-methyl-

benzaldehyde, 3,4-dimethoxy-

cyclohexanol

ethene, 1,1-difluoro-

2,4-pyrimidinediamine, 6-(1-piperidinyl)-, 3-oxide acetic acid, propyl ester

furo[3',4':6,7]naphtho[2,3-d]-1,3-dioxol-6(5aH)-one,

5,8,8a,9-tetrahydro-5-(4-hydroxy-3,5-

dimethoxyphenyl)-9-[[4,6-o-[2-thienylmethylene]-

beta-glucopyranosyl]oxy]

pyrazine, 2-methoxy-3-methyl-

1,3-pentanediol, 2,2,4-trimethyl-

benzenamine, 4-ethoxy-

ethanedione, di-2-furanyl-

hydrazine, phenyl-

methane, dichloro-

2-propenoic acid, oxybis(2,1-ethanediyloxy-2,1ethanediyl) ester

2(1H)-quinolinone

5,12-naphthacenedione, 10-[(3-amino-2,3,6-

trideoxy-alpha-hexopyranosyl)oxy]-7,8,9,10-

tetrahydro-6,8,11-trihydroxy-8-(hydroxyacetyl)-1-

methoxy-

benzamide, 2-hydroxy-

2,5-cyclohexadiene-1,4-dione, 2,5-dimethyl-
$\mathrm{O}(\mathrm{c}$

$\mathrm{O}=\mathrm{C}(\mathrm{NC}(\mathrm{C}(\mathrm{O}) \mathrm{c}(\operatorname{ccc}(\mathrm{N}(=\mathrm{O})$

$(=\mathrm{O})) \mathrm{c} 1) \mathrm{c} 1) \mathrm{CO}) \mathrm{C}(\mathrm{Cl}) \mathrm{Cl}$

$\mathrm{O}=\mathrm{S}(=\mathrm{O})(\mathrm{OCC}) \mathrm{OCC}$

$\mathrm{O}=\mathrm{S}(=\mathrm{O})(\mathrm{N}) \mathrm{c}(\mathrm{ccc}(\mathrm{c} 1) \mathrm{Cl}) \mathrm{c} 1$

$\mathrm{c} 1 \mathrm{c}(\mathrm{O}) \mathrm{c}(\mathrm{O}) \operatorname{ccc} 1 \mathrm{C}=\mathrm{CC}(=\mathrm{O}) \mathrm{O}$

$\mathrm{O}=\mathrm{CNc}(\mathrm{cccc} 1) \mathrm{c} 1$

$\mathrm{O}(\mathrm{c}(\mathrm{c}(\mathrm{O}) \mathrm{c}(\mathrm{OC}) \mathrm{cc} 1) \mathrm{c} 1) \mathrm{C}$

$\mathrm{O}=\mathrm{CC}=\mathrm{CC}(\mathrm{OC}=\mathrm{C} 1)=\mathrm{C} 1$

$\mathrm{O}=\mathrm{C}(\mathrm{NC}(=\mathrm{O}) \mathrm{c} 1 \mathrm{cccc} 2) \mathrm{c} 12$

$\mathrm{O}=\mathrm{C}(\mathrm{OCCOCCO}) \mathrm{c}(\operatorname{cccc} 1) \mathrm{c} 1$

$\mathrm{O}=\mathrm{C}(\mathrm{Oc}(\mathrm{c} 1 \mathrm{ccc} 2) \mathrm{c} 2) \mathrm{N} 1$

$\mathrm{O}=\mathrm{C}(\mathrm{Nc}(\mathrm{cccc} 1) \mathrm{c} 1) \mathrm{C}$

$\mathrm{O}(\mathrm{c}(\mathrm{cccc} 1) \mathrm{c} 1) \mathrm{CCO}$

$\mathrm{O}(\mathrm{N}(=\mathrm{O})=\mathrm{O}) \mathrm{CCO}(\mathrm{N}(=\mathrm{O})=\mathrm{O})$

$\mathrm{OCCC}(\mathrm{C}) \mathrm{C}$

$\mathrm{O}=\mathrm{C}(\mathrm{O}) \mathrm{CC}(\mathrm{C}) \mathrm{C}$

$\mathrm{OC}(=\mathrm{O}) \operatorname{cCc} 1 \mathrm{ccc}(\mathrm{O}) \mathrm{cc} 1$

$\mathrm{n}(\operatorname{cccc} 1 \mathrm{C}(\mathrm{N}(\mathrm{CC} 2) \mathrm{C}) \mathrm{C} 2) \mathrm{c} 1$

$\mathrm{n} 1 \mathrm{c}(\mathrm{SC}) \mathrm{cncc} 1$

$\mathrm{O}(\mathrm{c}(\mathrm{c}(\mathrm{N}) \mathrm{ccc} 1) \mathrm{c} 1) \mathrm{C}$

$\mathrm{FC}(\mathrm{F})(\mathrm{F}) \mathrm{F}$

$\mathrm{O}=\mathrm{C}(\mathrm{O}) \mathrm{C}(\mathrm{CC}) \mathrm{C}$

$\mathrm{O}=\mathrm{C}(\mathrm{Oc}(\mathrm{c}(\operatorname{ccc} 1) \mathrm{C}(=\mathrm{O}) \mathrm{O}) \mathrm{c} 1) \mathrm{C}$

$\mathrm{BrC}$

$\mathrm{CC}(\mathrm{CCC}) \mathrm{O}$

$\mathrm{COc} 1 \mathrm{ccc} 2 \mathrm{CC} 5 \mathrm{C} 3 \mathrm{C}=\mathrm{CC}(\mathrm{O}) \mathrm{C} 4 \mathrm{Oc} 1 \mathrm{c} 2$

C34CCN5C

$\mathrm{Clc} 1 \operatorname{ccc} 2 \mathrm{~N}=\mathrm{C}(\mathrm{C}) \mathrm{NS}(=\mathrm{O})(=\mathrm{O}) \mathrm{c} 2 \mathrm{c} 1$

$\mathrm{O}=\mathrm{C}(\mathrm{C}(\mathrm{C})(\mathrm{C}) \mathrm{C}) \mathrm{C}$

$\mathrm{n}(\operatorname{cccc} 1 \mathrm{C}) \mathrm{c} 1$

$\operatorname{CcC}(\mathrm{N}(=\mathrm{O})(=0)) \mathrm{C}$

$\mathrm{O}=\mathrm{C}(\mathrm{OCC}) \mathrm{CCC}(=\mathrm{O}) \mathrm{OCC}$

$\mathrm{O}(\mathrm{C}(\mathrm{OCC}) \mathrm{OCC}) \mathrm{CC}$

$\mathrm{O}=\mathrm{Cc}(\operatorname{ccc}(\mathrm{O}) \mathrm{c} 1 \mathrm{OC}) \mathrm{c} 1$

$\mathrm{O}=\mathrm{C}(\mathrm{OCC}) \mathrm{OCC}$

$\mathrm{OC}(\mathrm{CC}) \mathrm{CC}$

$\mathrm{O}=\mathrm{C}(\mathrm{O}) \mathrm{c}(\mathrm{c}(\mathrm{N}) \mathrm{ccc} 1) \mathrm{c} 1$

$\mathrm{O}=\mathrm{C}(\mathrm{OCC}) \mathrm{CC}$

$\mathrm{C} 1=\mathrm{CN}(\mathrm{C}) \mathrm{C}=\mathrm{C} 1$

$\mathrm{n}(\operatorname{ccc}(\mathrm{c} 1) \mathrm{C}) \mathrm{c} 1$

$\mathrm{O}=\mathrm{Cc}(\operatorname{ccc}(\mathrm{OC}) \mathrm{c} 1 \mathrm{OC}) \mathrm{c} 1$

$\mathrm{OC}(\mathrm{CCCC} 1) \mathrm{C} 1$

$\mathrm{FC}(\mathrm{F})=\mathrm{C}$

$\mathrm{C} 1 \mathrm{CCCCN} 1 \mathrm{c} 2 \mathrm{nc}(\mathrm{N}) \mathrm{n}(=\mathrm{O}) \mathrm{c}(\mathrm{N}) \mathrm{c} 2$

$\mathrm{O}=\mathrm{C}(\mathrm{OCCC}) \mathrm{C}$

$\mathrm{COc} 1 \mathrm{cc}(\mathrm{cc}(\mathrm{OC}) \mathrm{c} 1 \mathrm{OC}) \mathrm{C} 2 \mathrm{C} 3 \mathrm{C}(\mathrm{COC} 3$

$=0) \mathrm{C}(\mathrm{OC} 4 \mathrm{OC} 5 \mathrm{COC}(\mathrm{OC} 5 \mathrm{C}(\mathrm{O}) \mathrm{C} 4 \mathrm{O}) \mathrm{c}$

$6 \mathrm{cccs} 6) \mathrm{c} 7 \mathrm{cc} 8 \mathrm{OCOc} 8 \mathrm{cc} 27$

$\mathrm{O}(\mathrm{c}(\mathrm{nccn} 1) \mathrm{c} 1 \mathrm{C}) \mathrm{C}$

$\mathrm{OCC}(\mathrm{C}(\mathrm{O}) \mathrm{C}(\mathrm{C}) \mathrm{C})(\mathrm{C}) \mathrm{C}$

$\mathrm{O}(\mathrm{c}(\operatorname{ccc}(\mathrm{N}) \mathrm{c} 1) \mathrm{c} 1) \mathrm{CC}$

$01 \operatorname{cccc} 1 \mathrm{C}(=\mathrm{O}) \mathrm{C}(=\mathrm{O}) \mathrm{c} 2 \mathrm{occc} 2$

$\mathrm{N}(\mathrm{N}) \mathrm{c}(\mathrm{cccc} 1) \mathrm{c} 1$

$\mathrm{ClCCl}$

$\mathrm{O}=\mathrm{C}(\mathrm{OCcoc} \operatorname{coc} \operatorname{coc} \operatorname{coc}(=\mathrm{O}) \mathrm{C}=$

$\mathrm{C}) \mathrm{C}=\mathrm{C}$

$\mathrm{c} 1 \mathrm{ccc} 2 \mathrm{C}=\mathrm{CC}(=\mathrm{O}) \mathrm{Nc} 2 \mathrm{c} 1$

$\operatorname{coc} 4 \operatorname{cccc} 5 \mathrm{C}(=\mathrm{O}) \mathrm{c} 3 \mathrm{c}(\mathrm{O}) \mathrm{c} 2 \mathrm{CC}(\mathrm{O})$

$(\mathrm{CC}(\mathrm{OC} 1 \mathrm{CC}(\mathrm{N}) \mathrm{C}(\mathrm{O}) \mathrm{C}(\mathrm{C}) \mathrm{O} 1) \mathrm{c} 2 \mathrm{c}(\mathrm{O}) \mathrm{c}$

$3 \mathrm{C}(=\mathrm{O}) \mathrm{c} 45) \mathrm{C}(=\mathrm{O}) \mathrm{CO}$

$\mathrm{O}=\mathrm{C}(\mathrm{N}) \mathrm{c}(\mathrm{c}(\mathrm{O}) \mathrm{ccc} 1) \mathrm{c} 1$

$\mathrm{O}=\mathrm{C} 1 \mathrm{C}(\mathrm{C})=\mathrm{CC}(=\mathrm{O}) \mathrm{C}(\mathrm{C})=\mathrm{C} 1$

$\begin{array}{lllll}1.13 & 1.16 & 0.03 & 1.23 & 0.10 \\ 1.13 & 1.27 & 0.14 & 0.90 & -0.23\end{array}$

$\begin{array}{lllll}1.14 & 0.92 & -0.22 & 1.15 & 0.01\end{array}$

$\begin{array}{lllll}1.14 & 1.14 & 0.00 & -0.29 & -1.43\end{array}$

$\begin{array}{lllll}1.15 & 1.01 & -0.14 & 1.04 & -0.11\end{array}$

$\begin{array}{lllll}1.15 & 1.11 & -0.04 & 1.67 & 0.52\end{array}$

$\begin{array}{lllll}1.15 & 1.13 & -0.02 & 1.20 & 0.05\end{array}$

$\begin{array}{lllll}1.15 & 1.16 & 0.01 & 1.29 & 0.14\end{array}$

$\begin{array}{lllll}1.15 & 1.19 & 0.04 & 1.17 & 0.02\end{array}$

$\begin{array}{lllll}1.15 & 1.30 & 0.15 & 0.83 & -0.32\end{array}$

$\begin{array}{lllll}1.16 & 0.58 & -0.58 & 1.07 & -0.09\end{array}$

$\begin{array}{lllll}1.16 & 0.95 & -0.21 & 1.37 & 0.21\end{array}$

$\begin{array}{lllll}1.16 & 1.10 & -0.06 & 1.05 & -0.11\end{array}$

$\begin{array}{lllll}1.16 & 1.10 & -0.06 & 1.22 & 0.06\end{array}$

$\begin{array}{lllll}1.16 & 1.17 & 0.01 & 1.17 & 0.01\end{array}$

$\begin{array}{lllll}1.16 & 1.26 & 0.10 & 1.33 & 0.17\end{array}$

$\begin{array}{lllll}1.16 & 1.49 & 0.33 & 1.26 & 0.10\end{array}$

$\begin{array}{lllll}1.16 & 1.81 & 0.65 & 1.15 & -0.01\end{array}$

$\begin{array}{lllll}1.17 & 1.00 & -0.17 & 0.87 & -0.30\end{array}$

$\begin{array}{lllll}1.17 & 1.08 & -0.09 & 0.99 & -0.18\end{array}$

$\begin{array}{lllll}1.18 & 1.16 & -0.02 & 0.93 & -0.25\end{array}$

$\begin{array}{lllll}1.18 & 1.19 & 0.01 & 1.75 & 0.57\end{array}$

$\begin{array}{lllll}1.18 & 1.49 & 0.31 & 1.47 & 0.29\end{array}$

$\begin{array}{lllll}1.19 & 1.13 & -0.06 & 1.43 & 0.24\end{array}$

$\begin{array}{lllll}1.19 & 1.18 & -0.01 & 0.68 & -0.51\end{array}$

$\begin{array}{lllll}1.19 & 1.26 & 0.07 & 1.18 & -0.01\end{array}$

$\begin{array}{lllll}1.19 & 1.28 & 0.09 & 1.20 & 0.01\end{array}$

$\begin{array}{lllll}1.20 & 1.01 & -0.19 & 0.96 & -0.24\end{array}$

$\begin{array}{lllll}1.20 & 1.13 & -0.07 & 1.59 & 0.39\end{array}$

$\begin{array}{lllll}1.20 & 1.35 & 0.15 & 1.11 & -0.09\end{array}$

$\begin{array}{lllll}1.20 & 1.36 & 0.16 & 1.49 & 0.29\end{array}$

$\begin{array}{lllll}1.20 & 1.39 & 0.19 & 1.25 & 0.05\end{array}$

$\begin{array}{lllll}1.20 & 1.45 & 0.25 & 0.99 & -0.21\end{array}$

$\begin{array}{lllll}1.21 & 1.05 & -0.16 & 1.31 & 0.10\end{array}$

$\begin{array}{lllll}1.21 & 1.22 & 0.01 & 0.86 & -0.35\end{array}$

$\begin{array}{lllll}1.21 & 1.26 & 0.05 & 1.22 & 0.01\end{array}$

$\begin{array}{lllll}1.21 & 1.36 & 0.15 & 0.78 & -0.43\end{array}$

$\begin{array}{lllll}1.21 & 1.36 & 0.15 & 1.32 & 0.11\end{array}$

$\begin{array}{lllll}1.21 & 1.43 & 0.22 & 1.31 & 0.10\end{array}$

$\begin{array}{lllll}1.22 & 1.35 & 0.13 & 1.14 & -0.08\end{array}$

$\begin{array}{lllll}1.22 & 1.36 & 0.14 & 1.64 & 0.42\end{array}$

$\begin{array}{lllll}1.23 & 1.64 & 0.41 & 1.35 & 0.12\end{array}$

$\begin{array}{lllll}1.24 & 1.24 & 0.00 & 1.56 & 0.32\end{array}$

$\begin{array}{lllll}1.24 & 1.35 & 0.11 & 1.24 & 0.00\end{array}$

$\begin{array}{lllll}1.24 & 1.36 & 0.12 & 1.28 & 0.04\end{array}$

$\begin{array}{lllll}1.24 & 1.42 & 0.18 & 1.97 & 0.73\end{array}$

1.420 .180 .1 .970

$\begin{array}{lcccc}1.24 & 1.46 & 0.22 & 1.09 & -0.15 \\ 1.24 & 1.49 & 0.25 & 1.08 & -0.16 \\ 1.24 & 1.65 & 0.41 & 1.64 & 0.40 \\ 1.24 & 1.80 & 0.56 & 1.55 & 0.31 \\ 1.25 & 0.79 & -0.46 & 0.95 & -0.30 \\ 1.25 & 1.34 & 0.09 & 1.12 & -0.13 \\ 1.26 & 0.29 & -0.97 & 1.10 & -0.16 \\ & & & & \\ 1.26 & 1.32 & 0.06 & 1.51 & 0.25 \\ 1.27 & 1.85 & 0.58 & 1.41 & 0.14 \\ & & & & \\ & & & & \\ 1.28 & 1.03 & -0.25 & 0.74 & -0.54 \\ 1.28 & 1.34 & 0.06 & 1.11 & -0.17\end{array}$


4,4-bipyridine

pyrazine, tetramethyl-

1-butanol, 2-methyl-

butanoic acid, methyl ester

acetamide, N-(5,6,7,9-tetrahydro-1,2,3,10-

tetramethoxy-9-oxobenzo[a]heptalen-7-yl)-

glucitol, 1,4:3,6-dianhydro-, dinitrate

2-pentanone, 4-methyl-

quinoxaline

3-pyridinecarboxylic acid, ethyl ester

2-propenoic acid, ethyl ester

phenol, 2-methoxy-

benzenamine, 2-methyl-

acetic acid, trichloro-

phenol, 2,4,6-trinitro-

2,4-hexadienoic acid, (E,E)-

4-pyridinamine, N,N-dimethyl-

acetic acid, phenoxy-

furan

1,4-naphthalenedione, 2-methoxy-

benzaldehyde, 4-hydroxy-

3-buten-2-one, 4-(2-furanyl)-

4-thia-1-azabicyclo[3.2.0]heptane-2-carboxylic acid,

6-[(aminophenylacetyl)amino]-3,3-dimethyl-7-oxo

benzeneethanol

benzenamine, 3-nitro-

benzaldehyde, 2-hydroxy-3-methoxy-

2-hexanone

2-propenoic acid, 2-methyl-, methyl ester

cyclohexanone, 4-methyl-

phenol, 2-methoxy-, acetate

pyrimido[1,2-a]azepine, $2,3,4,6,7,8,9,10$-octahydro-

1,2-propanediol, 3-(2-methoxyphenoxy)-

benzenamine, 4-nitro-

2H-1-benzopyran-2-one

pentanoic acid

benzenamine, 4-methyl-

$2 \mathrm{H}-1,3,5$-thiadiazine-2-thione, tetrahydro-3,5-

dimethyl-

carbamic acid, [1,2-

phenylenebis(iminocarbonothioyl)]bis-, dimethyl

ester

benzonitrile, 2-amino-

benzenamine, 3-methyl-

2-propanamine, N-(1-methylethyl)-

1,4-benzenediol, 2-chloro-

hydrazine, (4-nitrophenyl)-

benzeneethanamine

methane, bromochloro-

benzeneacetic acid

$1 \mathrm{H}$-indole-3-acetic acid

acetic acid, phenoxy-, methyl ester

benzenemethanol, alpha-methyl-

benzeneacetic acid, 4-methoxy-

ethane, chloro-

benzenethiol, 2-amino-

$1 \mathrm{H}$-benzotriazole

ethanone, 1-(methylphenyl)-

ethanol, 2-chloro-, phosphate (3:1)

ethanamine, $\mathrm{N}, \mathrm{N}$-diethyl-

1,2-propadiene

1,3-butanedione, 4,4,4-trifluoro-1-(2-thienyl)-

benzoic acid, 2-nitro-

phenol

2-butyne

\begin{tabular}{|c|c|c|c|c|c|}
\hline $\mathrm{n}(\mathrm{ccc}(\mathrm{c} 1) \mathrm{c}(\mathrm{ccnc} 2) \mathrm{c} 2) \mathrm{c} 1$ & 1.28 & 1.38 & 0.10 & 1.40 & 0.12 \\
\hline $\mathrm{n}(\mathrm{c}(\mathrm{c}(\mathrm{nc} 1 \mathrm{C}) \mathrm{C}) \mathrm{C}) \mathrm{c} 1 \mathrm{C}$ & 1.28 & 2.13 & 0.85 & 1.46 & 0.18 \\
\hline $\mathrm{OCC}(\mathrm{CC}) \mathrm{C}$ & 1.29 & 1.26 & -0.03 & 1.24 & -0.05 \\
\hline $\mathrm{O}=\mathrm{C}(\mathrm{OC}) \mathrm{CCC}$ & 1.29 & 1.36 & 0.07 & 1.22 & -0.07 \\
\hline $\begin{array}{l}\mathrm{O}=\mathrm{C}(\mathrm{NC}(\mathrm{C}(\mathrm{C}(\mathrm{c}(\mathrm{c}(\mathrm{cc}(\mathrm{OC}) \mathrm{c} 1 \mathrm{OC}) \mathrm{C} 2) \mathrm{c} \\
1 \mathrm{OC})=\mathrm{CC}=\mathrm{C}(\mathrm{OC}) \mathrm{C} 3(=\mathrm{O}))=\mathrm{C} 3) \mathrm{C} 2) \mathrm{C}\end{array}$ & 1.30 & 1.86 & 0.56 & 1.59 & 0.29 \\
\hline $\begin{array}{l}\mathrm{O}(\mathrm{N}(=\mathrm{O})=\mathrm{O}) \mathrm{C}(\mathrm{C}(\mathrm{OCC} 1 \mathrm{O}(\mathrm{N}(=\mathrm{O})=\mathrm{O})) \\
\mathrm{C} 1 \mathrm{O} 2) \mathrm{C} 2\end{array}$ & 1.31 & 0.76 & -0.55 & 0.87 & -0.44 \\
\hline $\mathrm{O}=\mathrm{C}(\mathrm{CC}(\mathrm{C}) \mathrm{C}) \mathrm{C}$ & 1.31 & 1.16 & -0.15 & 1.31 & 0.00 \\
\hline$n(c(c(n c 1) c c c 2) c 2) c 1$ & 1.32 & 1.12 & -0.20 & 1.12 & -0.20 \\
\hline $\mathrm{O}=\mathrm{C}(\mathrm{OCC}) \mathrm{c}(\operatorname{cccn} 1) \mathrm{c} 1$ & 1.32 & 1.13 & -0.19 & 1.17 & -0.15 \\
\hline $\mathrm{O}=\mathrm{C}(\mathrm{OCC}) \mathrm{C}=\mathrm{C}$ & 1.32 & 1.22 & -0.10 & 1.24 & -0.08 \\
\hline $\mathrm{O}(\mathrm{c}(\mathrm{c}(\mathrm{O}) \mathrm{ccc} 1) \mathrm{c} 1) \mathrm{C}$ & 1.32 & 1.34 & 0.02 & 1.32 & 0.00 \\
\hline $\mathrm{Nc}(\mathrm{c}(\operatorname{ccc} 1) \mathrm{C}) \mathrm{c} 1$ & 1.32 & 1.62 & 0.30 & 1.32 & 0.00 \\
\hline $\mathrm{O}=\mathrm{C}(\mathrm{O}) \mathrm{C}(\mathrm{Cl})(\mathrm{Cl}) \mathrm{Cl}$ & 1.33 & 1.44 & 0.11 & 1.17 & -0.16 \\
\hline $\begin{array}{l}\mathrm{N}(=\mathrm{O})(=\mathrm{O}) \mathrm{c}(\mathrm{cc}(\mathrm{N}(=\mathrm{O}) \\
(=\mathrm{O})) \mathrm{c}(\mathrm{O}) \mathrm{c} 1 \mathrm{~N}(=\mathrm{O})(=\mathrm{O})) \mathrm{c} 1\end{array}$ & 1.33 & 1.54 & 0.21 & 1.83 & 0.50 \\
\hline $\mathrm{O}=\mathrm{C}(\mathrm{O}) \mathrm{C}=\mathrm{CC}=\mathrm{CC}$ & 1.33 & 1.62 & 0.29 & 1.77 & 0.44 \\
\hline$n(\operatorname{ccc}(N(C) C) c 1) c 1$ & 1.34 & 0.98 & -0.36 & 1.36 & 0.02 \\
\hline $\mathrm{O}=\mathrm{C}(\mathrm{O}) \operatorname{COc}(\operatorname{cccc} 1) \mathrm{c} 1$ & 1.34 & 1.33 & -0.01 & 1.48 & 0.14 \\
\hline $\mathrm{O} 1 \mathrm{C}=\mathrm{CC}=\mathrm{C} 1$ & 1.34 & 1.36 & 0.02 & 1.24 & -0.10 \\
\hline $\operatorname{c} 1 \operatorname{ccc} 2 \mathrm{C}(=\mathrm{O}) \mathrm{C}=\mathrm{C}(\mathrm{OC}) \mathrm{C}(=\mathrm{O}) \mathrm{c} 2 \mathrm{c} 1$ & 1.35 & 0.95 & -0.40 & 1.70 & 0.35 \\
\hline $\mathrm{O}=\mathrm{Cc}(\operatorname{ccc}(\mathrm{O}) \mathrm{c} 1) \mathrm{c} 1$ & 1.35 & 1.23 & -0.12 & 1.27 & -0.08 \\
\hline $\mathrm{O}=\mathrm{C}(\mathrm{C}=\mathrm{CC}(\mathrm{OC}=\mathrm{C} 1)=\mathrm{C} 1) \mathrm{C}$ & 1.35 & 1.41 & 0.06 & 1.42 & 0.07 \\
\hline $\begin{array}{l}\mathrm{CC} 3(\mathrm{C}) \mathrm{SC} 2 \mathrm{C}(\mathrm{NC}(=\mathrm{O}) \mathrm{C}(\mathrm{N}) \mathrm{c} 1 \mathrm{ccccc} 1) \\
\mathrm{C}(=\mathrm{O}) \mathrm{N} 2 \mathrm{C} 3 \mathrm{C}(=\mathrm{O})(\mathrm{O})\end{array}$ & 1.35 & 1.45 & 0.10 & 0.88 & -0.47 \\
\hline $\begin{array}{l}\mathrm{C}(=\mathrm{O}) \mathrm{N} 2 \mathrm{C} 3 \mathrm{C}(=\mathrm{O})(\mathrm{O}) \\
\mathrm{OCCc}(\operatorname{cccc} 1) \mathrm{c} 1\end{array}$ & 1.36 & 1.57 & 0.21 & 1.51 & 0.15 \\
\hline $\mathrm{N}(=\mathrm{O})(=\mathrm{O}) \mathrm{c}(\operatorname{cccc} 1 \mathrm{~N}) \mathrm{c} 1$ & 1.37 & 1.47 & 0.10 & 1.53 & 0.1 \\
\hline $\mathrm{O}=\mathrm{Cc}(\mathrm{c}(\mathrm{O}) \mathrm{c}(\mathrm{OC}) \mathrm{cc} 1) \mathrm{c} 1$ & 1.37 & 1.83 & 0.46 & 1.31 & -0.06 \\
\hline $\mathrm{O}=\mathrm{C}(\mathrm{CCCC}) \mathrm{C}$ & 1.38 & 1.24 & -0.14 & 1.45 & 0.07 \\
\hline $\mathrm{O}=\mathrm{C}(\mathrm{OC}) \mathrm{C}(=\mathrm{C}) \mathrm{C}$ & 1.38 & 1.28 & -0.10 & 1.10 & -0.28 \\
\hline $\mathrm{O}=\mathrm{C}(\mathrm{CCC}(\mathrm{C} 1) \mathrm{C}) \mathrm{C} 1$ & 1.38 & 1.54 & 0.16 & 1.46 & 0.08 \\
\hline $\operatorname{COc} 1 \operatorname{ccccc} 10 \mathrm{OC}(=\mathrm{O}) \mathrm{C}$ & 1.38 & 1.67 & 0.29 & 1.64 & 0.26 \\
\hline $\mathrm{N}(=\mathrm{C}(\mathrm{N}(\mathrm{CCCC} 1) \mathrm{CC} 2) \mathrm{C} 1) \mathrm{C} 2$ & 1.38 & 2.70 & 1.32 & 1.60 & 0.2 \\
\hline $\mathrm{O}(\mathrm{c}(\mathrm{c}(\mathrm{OC}) \mathrm{ccc} 1) \mathrm{c} 1) \mathrm{CC}(\mathrm{O}) \mathrm{CO}$ & 1.39 & 0.42 & -0.97 & 0.76 & -0.63 \\
\hline $\mathrm{N}(=\mathrm{O})(=\mathrm{O}) \mathrm{c}(\operatorname{ccc}(\mathrm{N}) \mathrm{c} 1) \mathrm{c} 1$ & 1.39 & 1.47 & 0.08 & 1.50 & 0.11 \\
\hline $\operatorname{c1cc2OC}(=\mathrm{O}) \mathrm{C}=\mathrm{Cc} 2 \mathrm{cc} 1$ & 1.39 & 1.51 & 0.12 & 1.72 & 0.33 \\
\hline $\mathrm{O}=\mathrm{C}(\mathrm{O}) \mathrm{CCCC}$ & 1.39 & 1.56 & 0.17 & 1.34 & -0.0 \\
\hline $\mathrm{Cc} 1 \mathrm{ccc}(\mathrm{N}) \operatorname{cc} 1$ & 1.39 & 1.62 & 0.23 & 1.34 & -0.05 \\
\hline $\mathrm{N}(\mathrm{CSC}(\mathrm{N} 1 \mathrm{C})=\mathrm{S})(\mathrm{C} 1) \mathrm{C}$ & 1.40 & 0.94 & -0.46 & 0.48 & -0.9 \\
\hline $\begin{array}{l}\mathrm{COC}(=\mathrm{O}) \mathrm{NC}(=\mathrm{S}) \mathrm{Nc} 1 \operatorname{ccccc} 1 \mathrm{NC}(=\mathrm{S}) \mathrm{N} \\
\mathrm{C}(=\mathrm{O}) \mathrm{OC}\end{array}$ & 1.40 & 1.10 & -0.30 & 1.57 & 0.17 \\
\hline $\mathrm{N \# Cc}(\mathrm{c}(\mathrm{N}) \mathrm{ccc} 1) \mathrm{c} 1$ & 1.40 & 1.17 & -0.23 & 1.05 & -0.3 \\
\hline $\mathrm{Nc}(\operatorname{ccc} 1 \mathrm{C}) \mathrm{c} 1$ & 1.40 & 1.62 & 0.22 & 1.32 & -0.08 \\
\hline$N(C(C) C) C(C) C$ & 1.40 & 1.64 & 0.24 & 1.12 & -0.2 \\
\hline $\mathrm{Oc}(\mathrm{c}(\mathrm{cc}(\mathrm{O}) \mathrm{c} 1) \mathrm{Cl}) \mathrm{c} 1$ & 1.40 & 1.68 & 0.28 & 1.62 & 0.2 \\
\hline $\mathrm{N}(=\mathrm{O})(=\mathrm{O}) \mathrm{c}(\operatorname{ccc}(\mathrm{NN}) \mathrm{c} 1) \mathrm{c} 1$ & 1.41 & 1.19 & -0.22 & 1.27 & -0.1 \\
\hline $\operatorname{NCCc}(\operatorname{cccc} 1) \mathrm{c} 1$ & 1.41 & 1.34 & -0.07 & 1.41 & 0.00 \\
\hline $\mathrm{BrCCl}$ & 1.41 & 1.43 & 0.02 & 1.27 & -0.14 \\
\hline $\mathrm{O}=\mathrm{C}(\mathrm{O}) \mathrm{Cc}(\operatorname{cccc} 1) \mathrm{c} 1$ & 1.41 & 1.43 & 0.02 & 1.72 & 0.3 \\
\hline $\mathrm{O}=\mathrm{C}(\mathrm{O}) \mathrm{CC}(\mathrm{c}(\mathrm{c}(\mathrm{N} 1) \mathrm{ccc} 2) \mathrm{c} 2)=\mathrm{C} 1$ & 1.41 & 1.49 & 0.08 & 1.87 & 0.46 \\
\hline $\mathrm{O}=\mathrm{C}(\mathrm{OC}) \mathrm{COc}(\operatorname{cccc} 1) \mathrm{c} 1$ & 1.41 & 1.61 & 0.20 & 1.90 & 0.4 \\
\hline $\mathrm{OC}(\mathrm{c}(\operatorname{cccc} 1) \mathrm{c} 1) \mathrm{C}$ & 1.42 & 1.49 & 0.07 & 1.58 & 0.16 \\
\hline $\mathrm{O}=\mathrm{C}(\mathrm{O}) \mathrm{Cc}(\operatorname{ccc}(\mathrm{OC}) \mathrm{c} 1) \mathrm{c} 1$ & 1.42 & 1.51 & 0.09 & 1.73 & 0.31 \\
\hline $\mathrm{CICC}$ & 1.43 & 1.58 & 0.15 & 1.47 & 0.04 \\
\hline $\mathrm{Sc}(\mathrm{c}(\mathrm{N}) \mathrm{ccc} 1) \mathrm{c} 1$ & 1.43 & 1.77 & 0.34 & 1.52 & $0.0 s$ \\
\hline c1ccc2nnnc2c1 & 1.44 & 1.17 & -0.27 & 1.19 & -0.2 \\
\hline$c(\operatorname{ccc} 1 \mathrm{CC}(=\mathrm{O}) \mathrm{C}) \operatorname{cc} 1$ & 1.44 & 1.47 & 0.03 & 1.70 & 0.2 \\
\hline $\mathrm{O}=\mathrm{P}(\mathrm{OCCCl})(\mathrm{OCCCl}) \mathrm{OCCCl}$ & 1.44 & 1.63 & 0.19 & 1.36 & -0.0 \\
\hline $\mathrm{N}(\mathrm{CC})(\mathrm{CC}) \mathrm{CC}$ & 1.45 & 1.51 & 0.06 & 1.57 & 0.1 \\
\hline$C=C=C$ & 1.45 & 1.65 & 0.20 & 1.67 & 0.22 \\
\hline $\mathrm{O}=\mathrm{C}(\mathrm{C}(\mathrm{SC}=\mathrm{C} 1)=\mathrm{C} 1) \mathrm{CC}(=\mathrm{O}) \mathrm{C}(\mathrm{F})(\mathrm{F}) \mathrm{F}$ & 1.46 & 0.84 & -0.62 & 2.37 & 0.9 \\
\hline $\mathrm{O}=\mathrm{C}(\mathrm{O}) \mathrm{c}(\mathrm{c}(\mathrm{N}(=\mathrm{O})(=\mathrm{O})) \mathrm{ccc} 1) \mathrm{c} 1$ & 1.46 & 1.35 & -0.11 & 1.50 & 0.0 \\
\hline $\mathrm{Oc}(\operatorname{cccc} 1) \mathrm{c} 1$ & 1.46 & 1.51 & 0.05 & 1.39 & -0.07 \\
\hline $\mathrm{C}(\# \mathrm{CC}) \mathrm{C}$ & 1.46 & 1.59 & 0.13 & 1.70 & 0.2 \\
\hline
\end{tabular}


benzene, 1,4-dinitro-

phosphoric acid, 2,2-dichloroethenyl dimethyl ester hydrazine, (2,4-dinitrophenyl)-

2,4,6(1H,3H,5H)-pyrimidinetrione, 5-ethyl-5-phenyl-

benzaldehyde, 3-nitro-

propanoic acid, 2,2-dimethyl-

benzaldehyde

ethane, 1,2-dichloro-

1-pentanamine

acetic acid, phenyl ester

cyclohexanamine

benzene, 1,3-dinitro-

2,5-cyclohexadiene-1,4-dione, dioxime

$1 \mathrm{H}$-benzimidazole, 5-nitro-

2,2-bipyridine

benzeneacetic acid, 3-methoxy-

1-pentanol

methane, iodo-

pyrazine, 2,3-diethyl-

bicyclo[2.2.1]heptane-2,3-dione, 1,7,7-trimethyl-

carbamic acid, $1 \mathrm{H}$-benzimidazol-2-yl-, methyl ester

propane, 2,2-oxybis-

phenol, 2-(1-methylethoxy)-, methylcarbamate

urea, N,N'-bis(2-chloroethyl)-N-nitroso-

benzene, 1,2,3-trimethoxy-

phenol, 2-amino-6-chloro-4-nitro-

2,3-naphthalenediamine

ethane, (methylthio)-

4H-1-benzopyran-4-one, 2-(2,4-dihydroxyphenyl)-

3,5,7-trihydroxy-

pyridine, 2-ethenyl-

methane, dichlorofluoro-

benzoic acid, 3,5-dinitro-

benzeneacetic acid, 4-fluoro-

1,3-benzenediol, 2-nitro-

benzaldehyde, 4-nitro-

benzonitrile

benzeneacetonitrile

$1 \mathrm{H}$-purin-6-amine, $\mathrm{N}$-(phenylmethyl)-

ethene, bromo-

nonanedioic acid

pyrazine, 2-methyl-3-propyl-

2H-1-benzopyran-2-one, 7-hydroxy-

benzoic acid, 4-hydroxy-

benzaldehyde, 3-ethoxy-4-hydroxy-

phenol, 4-methoxy-

benzenemethanol, 4-methyl-

acetamide, $\mathrm{N}$-(4-ethoxyphenyl)-

ethanone, 1-phenyl-

benzoic acid, 2-methoxy-

1,8-naphthyridine-3-carboxylic acid, 1-ethyl-1,4dihydro-7-methyl-4-oxo-

1H-imidazol-2-amine, N-(2,6-dichlorophenyl)-4,5dihydro-

benzenamine, 4,4-methylenebis-

benzonitrile, 4-hydroxy-

benzene, 1,2-dimethoxy-

1,2-benzenedicarboxylic acid, dimethyl ester

benzene, 2-methyl-1,3,5-trinitro-

1,3-isobenzofurandione

benzoic acid, 3,4-dimethoxy-

2-propanol, 1-phenoxy-

oxirane, phenyl-

pregn-4-ene-3,20-dione, 11,17,21-trihydroxy-, (11)- $\mathrm{O}=\mathrm{C}(\mathrm{C}(\mathrm{O})$
$\mathrm{N}(=\mathrm{O})(=\mathrm{O}) \mathrm{c}(\operatorname{ccc}(\mathrm{N}(=\mathrm{O})(=\mathrm{O})) \mathrm{c} 1) \mathrm{c} 1$

$\mathrm{O}=\mathrm{P}(\mathrm{OC})(\mathrm{OC}) \mathrm{OC}=\mathrm{C}(\mathrm{Cl}) \mathrm{Cl}$
$\mathrm{N}(=\mathrm{O})(=\mathrm{O}) \mathrm{c}(\mathrm{ccc}(\mathrm{NN}) \mathrm{c} 1 \mathrm{~N}(=\mathrm{O})(=\mathrm{O})) \mathrm{c} 1$

$\operatorname{CCC} 1(\mathrm{C}(=\mathrm{O}) \mathrm{NC}(=\mathrm{O}) \mathrm{NC} 1(=\mathrm{O})) \mathrm{c} 2 \mathrm{ccc}$ cc2

$\mathrm{O}=\mathrm{Cc}(\operatorname{cccc} 1 \mathrm{~N}(=\mathrm{O})(=\mathrm{O})) \mathrm{c} 1$

$\mathrm{O}=\mathrm{C}(\mathrm{O}) \mathrm{C}(\mathrm{C})(\mathrm{C}) \mathrm{C}$

$\mathrm{O}=\mathrm{Cc}(\operatorname{cccc} 1) \mathrm{c} 1$

$\mathrm{ClCCCl}$

$\mathrm{NCCCCC}$

$\mathrm{O}=\mathrm{C}(\mathrm{Oc}(\operatorname{cccc} 1) \mathrm{c} 1) \mathrm{C}$

$\mathrm{NC}(\mathrm{CCCC} 1) \mathrm{C} 1$

$\mathrm{N}(=\mathrm{O})(=\mathrm{O}) \mathrm{c}(\operatorname{cccc} 1 \mathrm{~N}(=\mathrm{O})(=\mathrm{O})) \mathrm{c} 1$

$\mathrm{ON}=\mathrm{C} 1 \mathrm{C}=\mathrm{CC}(=\mathrm{NO}) \mathrm{C}=\mathrm{C} 1$

$\mathrm{N}(=\mathrm{O})(=\mathrm{O}) \mathrm{c}(\operatorname{ccc}(\mathrm{ncn} 1) \mathrm{c} 12) \mathrm{c} 2$

$\mathrm{n}(\mathrm{c}(\mathrm{ccc} 1) \mathrm{c}(\mathrm{nccc} 2) \mathrm{c} 2) \mathrm{c} 1$

$\mathrm{OC}(=\mathrm{O}) \mathrm{Cc} 1 \mathrm{cc}(\mathrm{OC}) \operatorname{ccc} 1$

OCCCCC

$\mathrm{Cl}$

$\mathrm{n}(\mathrm{c}(\mathrm{c}(\mathrm{nc} 1) \mathrm{CC}) \mathrm{CC}) \mathrm{c} 1$

$\mathrm{CC} 1(\mathrm{C}) \mathrm{C} 2 \mathrm{CCC} 1(\mathrm{C}) \mathrm{C}(=\mathrm{O}) \mathrm{C} 2(=\mathrm{O})$

$\mathrm{c} 1 \mathrm{ccc} 2 \mathrm{nc}(\mathrm{NC}(=\mathrm{O}) \mathrm{OC}) \mathrm{nc} 2 \mathrm{c} 1$

$\mathrm{O}(\mathrm{C}(\mathrm{C}) \mathrm{C}) \mathrm{C}(\mathrm{C}) \mathrm{C}$

$\mathrm{CNC}(=\mathrm{O}) \mathrm{Oc} 1 \mathrm{ccccc} 1 \mathrm{OC}(\mathrm{C}) \mathrm{C}$

$\mathrm{CICCNC}(=\mathrm{O}) \mathrm{N}(\mathrm{CCCl}) \mathrm{N}=\mathrm{O}$

$\mathrm{O}(\mathrm{c}(\mathrm{c}(\mathrm{OC}) \mathrm{ccc} 1) \mathrm{c} 1 \mathrm{OC}) \mathrm{C}$

$\mathrm{N}(=\mathrm{O})(=\mathrm{O}) \mathrm{c}(\mathrm{cc}(\mathrm{N}) \mathrm{c}(\mathrm{O}) \mathrm{c} 1 \mathrm{Cl}) \mathrm{c} 1$

$\mathrm{c}(\mathrm{c}(\mathrm{ccc} 1) \mathrm{cc}(\mathrm{N}) \mathrm{c} 2 \mathrm{~N})(\mathrm{c} 1) \mathrm{c} 2$

$\mathrm{S}(\mathrm{CC}) \mathrm{C}$

$\mathrm{Oc} 1 \mathrm{cc}(\mathrm{O}) \mathrm{c} 2 \mathrm{C}(=\mathrm{O}) \mathrm{C}(\mathrm{O})=\mathrm{C}(\mathrm{c} 3 \operatorname{ccc}(\mathrm{O}) \mathrm{c}$ c3O)Oc2c1

$\mathrm{n}(\mathrm{c}(\mathrm{ccc} 1) \mathrm{C}=\mathrm{C}) \mathrm{c} 1$

$\mathrm{FC}(\mathrm{Cl}) \mathrm{Cl}$

$\mathrm{O}=\mathrm{C}(\mathrm{O}) \mathrm{c}(\operatorname{cc}(\mathrm{N}(=\mathrm{O})(=\mathrm{O})) \operatorname{cc} 1 \mathrm{~N}(=\mathrm{O})$

$(=\mathrm{O})) \mathrm{c} 1$

$\mathrm{O}=\mathrm{C}(\mathrm{O}) \mathrm{Cc}(\mathrm{ccc}(\mathrm{F}) \mathrm{c} 1) \mathrm{c} 1$

$\mathrm{N}(=\mathrm{O})(=\mathrm{O}) \mathrm{c}(\mathrm{c}(\mathrm{O}) \mathrm{ccc} 1) \mathrm{c} 1 \mathrm{O}$

$\mathrm{O}=\mathrm{Cc}(\operatorname{ccc}(\mathrm{N}(=\mathrm{O})(=\mathrm{O})) \mathrm{c} 1) \mathrm{c} 1$

$\mathrm{C}(\# \mathrm{~N}) \mathrm{c}(\mathrm{cccc} 1) \mathrm{c} 1$

$\mathrm{C}(\# \mathrm{~N}) \mathrm{Cc}(\operatorname{cccc} 1) \mathrm{c} 1$

$\mathrm{n}(\mathrm{c}(\mathrm{NCc}(\mathrm{cccc} 1) \mathrm{c} 1) \mathrm{c}(\mathrm{N}=\mathrm{CN} 2) \mathrm{c} 2 \mathrm{n} 3) \mathrm{c} 3$

$\mathrm{BrC}=\mathrm{C}$

$\mathrm{O}=\mathrm{C}(\mathrm{O}) \operatorname{ccccccc}(=0) \mathrm{O}$

$\mathrm{n}(\mathrm{c}(\mathrm{c}(\mathrm{nc} 1) \mathrm{CCC}) \mathrm{C}) \mathrm{c} 1$

$\mathrm{Oc} 1 \mathrm{ccc} 2 \mathrm{C}=\mathrm{CC}(=\mathrm{O}) \mathrm{Oc} 2 \mathrm{c} 1$

$\mathrm{O}=\mathrm{C}(\mathrm{O}) \mathrm{c}(\operatorname{ccc}(\mathrm{O}) \mathrm{c} 1) \mathrm{c} 1$

$\mathrm{O}=\mathrm{Cc}(\operatorname{ccc}(\mathrm{O}) \mathrm{c} 1 \mathrm{OCC}) \mathrm{c} 1$

$\mathrm{O}(\mathrm{c}(\mathrm{ccc}(\mathrm{O}) \mathrm{c} 1) \mathrm{c} 1) \mathrm{C}$

$\mathrm{OCc}(\operatorname{ccc}(\mathrm{c} 1) \mathrm{C}) \mathrm{c} 1$

$\mathrm{CC}(=\mathrm{O}) \mathrm{Nc} 1 \mathrm{ccc}(\mathrm{OCC}) \mathrm{cc} 1$

$\mathrm{O}=\mathrm{C}(\mathrm{c}(\operatorname{cccc} 1) \mathrm{c} 1) \mathrm{C}$

$\mathrm{O}=\mathrm{C}(\mathrm{O}) \mathrm{c}(\mathrm{c}(\mathrm{OC}) \mathrm{ccc} 1) \mathrm{c} 1$

$\mathrm{Cc} 1 \mathrm{ccc} 2 \mathrm{C}(=\mathrm{O}) \mathrm{C}(\mathrm{C}(=\mathrm{O}) \mathrm{O})=\mathrm{CN}(\mathrm{CC}) \mathrm{c}$ 2n1

$\mathrm{N} 1 \mathrm{C}(=\mathrm{Nc} 2 \mathrm{c}(\mathrm{Cl}) \mathrm{cccc} 2 \mathrm{Cl}) \mathrm{NCC} 1$

$\mathrm{Nc}(\operatorname{ccc}(\mathrm{c} 1) \mathrm{Cc}(\operatorname{ccc}(\mathrm{N}) \mathrm{c} 2) \mathrm{c} 2) \mathrm{c} 1$

$\mathrm{C}(\# \mathrm{~N}) \mathrm{c}(\mathrm{ccc}(\mathrm{O}) \mathrm{c} 1) \mathrm{c} 1$

$\mathrm{O}(\mathrm{c}(\mathrm{c}(\mathrm{OC}) \mathrm{ccc} 1) \mathrm{c} 1) \mathrm{C}$

$\mathrm{O}=\mathrm{C}(\mathrm{OC}) \mathrm{c}(\mathrm{c}(\mathrm{ccc} 1) \mathrm{C}(=\mathrm{O}) \mathrm{OC}) \mathrm{c} 1$

$\mathrm{N}(=\mathrm{O})(=\mathrm{O}) \mathrm{c}(\mathrm{cc}(\mathrm{N}(=\mathrm{O})$

$(=\mathrm{O})) \mathrm{c}(\mathrm{c} 1 \mathrm{~N}(=\mathrm{O})(=\mathrm{O})) \mathrm{C}) \mathrm{c} 1$

$\mathrm{O}=\mathrm{C}(\mathrm{OC}(=\mathrm{O}) \mathrm{c} 1 \mathrm{cccc} 2) \mathrm{c} 12$

$\mathrm{O}=\mathrm{C}(\mathrm{O}) \mathrm{c}(\operatorname{ccc}(\mathrm{OC}) \mathrm{c} 1 \mathrm{OC}) \mathrm{c} 1$

$\mathrm{O}(\mathrm{c}(\mathrm{cccc} 1) \mathrm{c} 1) \mathrm{CC}(\mathrm{O}) \mathrm{C}$

$\mathrm{O}(\mathrm{C} 1 \mathrm{c}(\mathrm{cccc} 2) \mathrm{c} 2) \mathrm{C} 1$

1.46

1.47

1.47

1.47

1.47

1.48

1.48

1.48

1.49

1.49

1.49

1.49

1.49

1.50

1.50

1.50

1.51

1.51

1.51

1.52

1.52

1.52

1.52

1.53

1.53

1.53

1.54

1.54

1.54

1.54

1.55

1.55

1.55

1.56

1.56

1.56

1.56

1.57

1.57

1.57

1.57

1.58

1.58

1.58

1.58

1.58

1.58

1.58

1.59

1.59

1.59

1.59

1.60

1.60

1.60

1.60

1.60

1.61

1.61

1.61

1.61
1.63

0.60

1.01

1.33

1.53

1.45

1.71

1.83

1.33

1.59

1.63

1.63

1.92

1.05

1.38

1.51

1.33

1.59

2.02

0.75

1.55

1.88

1.90

1.44

1.50

1.64

1.34

1.41

1.48

1.71

1.21

1.51

1.63

1.43

1.53

1.54

1.56

1.23

1.52

1.70

2.02

1.03

1.39

1.55

1.59

1.62

1.67

1.67

1.61

1.64

1.89

2.18

1.61

1.64

1.66

1.99

2.07

1.52

1.52

1.59

1.62

0.17

$-0.87$

$-0.46$

$-0.14$

0.06

$-0.03$

0.23

0.35

$-0.16$

0.10

0.14

0.14

0.43

$-0.45$

$-0.12$

0.01

$-0.18$

0.08

0.51

$-0.77$

0.03

0.36

0.38

$-0.09$

$-0.03$

0.11

$-0.20$

$-0.13$

$-0.06$

0.17

$-0.34$

$-0.04$

0.08

$-0.13$

$-0.03$

$-0.02$

0.00

$-0.34$

$-0.05$

0.13

0.45

$-0.55$ 
ethane, bromo-

pregna-1,4-diene-3,20-dione, 11,17,21-trihydroxy-, (11)-

1,2,3-propanetriol, trinitrate

3-quinolinamine

ethanone, 1-(2-aminophenyl)-

benzaldehyde, 4-ethoxy-3-methoxy-

1H-tetrazole, 5-phenyl-

methane, chlorotrifluoro-

2,4,6(1H,3H,5H)-pyrimidinetrione, 5-ethyl-5-(1-

methylpropyl)-

benzoic acid, 2-(acetylamino)-, methyl ester

3-hexanol

2-propanol, 1,1,1,3,3,3-hexafluoro-

benzenamine, $\mathrm{N}$-methyl-

acetamide, $\mathrm{N}$-(4-nitrophenyl)-

benzoic acid, 2-nitro-, methyl ester

1,3-benzenedicarboxylic acid

pyridine, 3-ethyl-

2h-benzimidazole-2-thione, 1,3-dihydro-

phenol, 2,4-dinitro-

1-propanamine, N-propyl-

pyridine, 2,6-dimethyl-

butanoic acid, 2-ethyl-

ethanol, 2-[2-(hexyloxy)ethoxy]-

methane, dibromo-

acetamide, $\mathrm{N}$-(4-methylphenyl)-

1,3-benzodioxol-4-ol, 2,2-dimethyl-,

methylcarbamate

2-cyclohexen-1-one, 3,5,5-trimethyl-

butanamide, N-[3-acetyl-4-[2-hydroxy-3-[(1-

methylethyl)amino]propoxy]phenyl]-

1,4-naphthalenedione

benzaldehyde, 3-methoxy-

benzoic acid, 2-hydroxy-3,5-dinitro-

cyclopropane

acetic acid, 1-methylpropyl ester

benzaldehyde, 2-methoxy-

ethanone, 1-(4-fluorophenyl)-

thioperoxydicarbonic diamide, tetramethyl-

cyclohexanol, 1-ethynyl-

benzaldehyde, 2-nitro-

2H-1-benzopyran-2-one, 7-methoxy-

ethanone, 1-(4-methoxyphenyl)-

benzoic acid, 2,5-dihydroxy-

benzenamine, 2-ethyl-

2-propanol, 1-(1H-indol-4-yloxy)-3-[(1-

methylethyl)amino]-

butanamide, N,N'-(3,3'-dimethyl[1,1'-biphenyl]-4,4'-

diyl)bis[3-oxo-]

piperidine, 1-ethyl-

acetic acid, 1,1-dimethylethyl ester

2-hexanol

benzeneethanamine, alpha-methyl-

benzaldehyde, 4-methoxy-

$2(3 \mathrm{H})$-benzothiazolone

1-propene

disulfide, dimethyl

1,8-naphthalenediamine

benzeneacetaldehyde

acetic acid, 2-methylpropyl ester

hexanal

acetic acid, butyl ester
$\mathrm{C}(\mathrm{C}(\mathrm{C}(\mathrm{C}(\mathrm{C}(\mathrm{C}(=\mathrm{CC}(=\mathrm{O}) \mathrm{C} 1) \mathrm{C} 2)$

$(\mathrm{C} 1) \mathrm{C}) \mathrm{C} 3 \mathrm{O}) \mathrm{C} 2) \mathrm{C} 4)(\mathrm{C} 3) \mathrm{C}) \mathrm{C} 4) \mathrm{CO}$

$\mathrm{BrCC}$

$\mathrm{C} 1 \mathrm{C} 2=\mathrm{CC}(=\mathrm{O}) \mathrm{C}=\mathrm{CC} 2(\mathrm{C}) \mathrm{C} 3 \mathrm{C}(\mathrm{O}) \mathrm{CC}$

$4(\mathrm{C}) \mathrm{C}(\mathrm{O})(\mathrm{C}(=\mathrm{O}) \mathrm{CO}) \mathrm{CCC} 4 \mathrm{C} 3 \mathrm{C} 1$

$\mathrm{O}(\mathrm{N}(=\mathrm{O})=\mathrm{O}) \mathrm{CC}(\mathrm{O}(\mathrm{N}(=\mathrm{O})=0)) \mathrm{CO}(\mathrm{N}($

$=\mathrm{O})=\mathrm{O})$

$\mathrm{n}(\mathrm{c}(\mathrm{c}(\mathrm{ccc} 1) \mathrm{cc} 2 \mathrm{~N}) \mathrm{c} 1) \mathrm{c} 2$

$\mathrm{O}=\mathrm{C}(\mathrm{c}(\mathrm{c}(\mathrm{N}) \mathrm{ccc} 1) \mathrm{c} 1) \mathrm{C}$

$\mathrm{O}=\mathrm{Cc}(\operatorname{ccc}(\mathrm{OCC}) \mathrm{c} 1 \mathrm{OC}) \mathrm{c} 1$

$\mathrm{N} 1 \mathrm{~N}=\mathrm{NN}=\mathrm{C} 1 \mathrm{c} 2 \mathrm{ccccc} 2$

$\mathrm{FC}(\mathrm{F})(\mathrm{F}) \mathrm{Cl}$

$\operatorname{CCC}(\mathrm{C}) \mathrm{C} 1(\mathrm{CC}) \mathrm{C}(=\mathrm{O}) \mathrm{NC}(=\mathrm{O}) \mathrm{NC} 1(=$ O)

$\mathrm{O}=\mathrm{C}(\mathrm{Nc}(\mathrm{c}(\mathrm{ccc} 1) \mathrm{C}(=\mathrm{O}) \mathrm{OC}) \mathrm{c} 1) \mathrm{C}$

$\mathrm{OC}(\mathrm{CCC}) \mathrm{CC}$

$\mathrm{FC}(\mathrm{F})(\mathrm{F}) \mathrm{C}(\mathrm{O}) \mathrm{C}(\mathrm{F})(\mathrm{F}) \mathrm{F}$

$\mathrm{N}(\mathrm{c}(\mathrm{cccc} 1) \mathrm{c} 1) \mathrm{C}$

$\mathrm{O}=\mathrm{C}(\mathrm{Nc}(\operatorname{ccc}(\mathrm{N}(=\mathrm{O})(=\mathrm{O})) \mathrm{c} 1) \mathrm{c} 1) \mathrm{C}$

$\mathrm{O}=\mathrm{C}(\mathrm{OC}) \mathrm{c}(\mathrm{c}(\mathrm{N}(=\mathrm{O})(=\mathrm{O})) \mathrm{ccc} 1) \mathrm{c} 1$

$\mathrm{O}=\mathrm{C}(\mathrm{O}) \mathrm{c}(\operatorname{cccc} 1 \mathrm{C}(=\mathrm{O}) \mathrm{O}) \mathrm{c} 1$

$\mathrm{n}(\mathrm{cccc} 1 \mathrm{CC}) \mathrm{c} 1$

$\mathrm{N}(\mathrm{c}(\mathrm{c}(\mathrm{N} 1) \mathrm{ccc} 2) \mathrm{c} 2)=\mathrm{C} 1 \mathrm{~S}$

$\mathrm{N}(=\mathrm{O})(=\mathrm{O}) \mathrm{c}(\operatorname{ccc}(\mathrm{O}) \mathrm{c} 1 \mathrm{~N}(=\mathrm{O})(=\mathrm{O})) \mathrm{c} 1$

$\mathrm{N}(\mathrm{CCC}) \mathrm{CCC}$

$\mathrm{n}(\mathrm{c}(\mathrm{ccc} 1) \mathrm{C}) \mathrm{c} 1 \mathrm{C}$

$\mathrm{O}=\mathrm{C}(\mathrm{O}) \mathrm{C}(\mathrm{CC}) \mathrm{CC}$

$\mathrm{O}(\mathrm{CCOCCO}) \mathrm{CCCCCC}$

$\mathrm{BrCBr}$

$\mathrm{O}=\mathrm{C}(\mathrm{Nc}(\operatorname{ccc}(\mathrm{c} 1) \mathrm{C}) \mathrm{c} 1) \mathrm{C}$

$\mathrm{CNC}(=\mathrm{O}) \mathrm{Oc} 1 \mathrm{cccc} 2 \mathrm{OC}(\mathrm{C})(\mathrm{C}) \mathrm{Oc} 12$

1.61
1.62

1.67

0.06

1.64

0.03

$\begin{array}{lllll}1.62 & 1.51 & -0.11 & 1.25 & -0.37\end{array}$

1.63

1.63

1.23

$-0.40$

1.57

$-0.06$

$\begin{array}{llll}1.57 & -0.06 & 0.98 & -0.65\end{array}$

$\begin{array}{llll}1.85 & 0.22 & 2.18 & 0.55\end{array}$

$\begin{array}{llll}0.18 & -1.47 & 1.14 & -0.51\end{array}$

$\begin{array}{llll}1.50 & -0.15 & 1.80 & 0.15\end{array}$

$\begin{array}{llll}1.51 & -0.14 & 1.70 & 0.05\end{array}$

$\begin{array}{llll}1.73 & 0.08 & 1.46 & -0.19\end{array}$

$\begin{array}{llll}1.75 & 0.10 & 1.76 & 0.11\end{array}$

$\begin{array}{llll}1.11 & -0.55 & 2.58 & 0.92\end{array}$

$\begin{array}{llll}1.62 & -0.04 & 1.68 & 0.02\end{array}$

$\begin{array}{llll}1.64 & -0.02 & 1.19 & -0.47\end{array}$

$\begin{array}{llll}1.65 & -0.01 & 1.71 & 0.05\end{array}$

$\begin{array}{llll}1.76 & 0.10 & 1.04 & -0.62\end{array}$

$\begin{array}{llll}1.84 & 0.18 & 1.63 & -0.03\end{array}$

$\begin{array}{llll}1.93 & 0.27 & 2.20 & 0.54\end{array}$

$\begin{array}{llll}1.73 & 0.06 & 1.89 & 0.22\end{array}$

$\begin{array}{llll}1.79 & 0.12 & 1.74 & 0.07\end{array}$

$\begin{array}{llll}1.79 & 0.12 & 1.74 & 0.07 \\ 1.90 & 0.22 & 1.60 & -0.08\end{array}$

$\begin{array}{llll}1.98 & 0.30 & 1.74 & 0.06\end{array}$

$\begin{array}{llll}1.27 & -0.43 & 1.65 & -0.05\end{array}$

$\begin{array}{llll}1.52 & -0.18 & 1.48 & -0.22\end{array}$

$\begin{array}{llll}1.52 & -0.18 & 1.48 & -0.22 \\ 1.65 & -0.05 & 1.28 & -0.42\end{array}$

$\begin{array}{llll}2.55 & 0.85 & 1.53 & -0.17\end{array}$

$\mathrm{O}=\mathrm{C}(\mathrm{C}=\mathrm{C}(\mathrm{CC} 1(\mathrm{C}) \mathrm{C}) \mathrm{C}) \mathrm{C} 1$

$\begin{array}{llll}2.62 & 0.92 & 1.90 & 0.20\end{array}$

$\mathrm{CC}(=\mathrm{O}) \mathrm{c} 1 \mathrm{cc}(\mathrm{NC}(=\mathrm{O}) \mathrm{CCC}) \operatorname{ccc} 10 \mathrm{OCC}\left(\begin{array}{lllll}1.71 & 1.19 & -0.52 & 1.43 & -0.28\end{array}\right.$

O) $\mathrm{CNC}(\mathrm{C}) \mathrm{C}$

$\mathrm{c} 1 \mathrm{cc} 2 \mathrm{C}(=\mathrm{O}) \mathrm{C}=\mathrm{CC}(=\mathrm{O}) \mathrm{c} 2 \mathrm{cc} 1$

$\mathrm{O}=\mathrm{Cc}(\operatorname{cccc} 10 \mathrm{OC}) \mathrm{c} 1$

$\mathrm{O}=\mathrm{C}(\mathrm{O}) \mathrm{c}(\mathrm{c}(\mathrm{O}) \mathrm{c}(\mathrm{N}(=\mathrm{O})(=\mathrm{O})) \operatorname{cc} 1 \mathrm{~N}(=\mathrm{O}) \quad 1.71$

$(=0)) c 1$

$\mathrm{C}(\mathrm{C} 1) \mathrm{C} 1$

$\mathrm{O}=\mathrm{C}(\mathrm{OC}(\mathrm{CC}) \mathrm{C}) \mathrm{C}$

$\mathrm{O}=\mathrm{Cc}(\mathrm{c}(\mathrm{OC}) \mathrm{ccc} 1) \mathrm{c} 1$

$\mathrm{O}=\mathrm{C}(\mathrm{c}(\operatorname{ccc}(\mathrm{F}) \mathrm{c} 1) \mathrm{c} 1) \mathrm{C}$

$\mathrm{N}(\mathrm{C}(=\mathrm{S}) \operatorname{SSC}(\mathrm{N}(\mathrm{C}) \mathrm{C})=\mathrm{S})(\mathrm{C}) \mathrm{C}$

$\mathrm{OC}(\mathrm{CHC})(\mathrm{CCCC} 1) \mathrm{C} 1$

$\mathrm{O}=\mathrm{Cc}(\mathrm{c}(\mathrm{N}(=\mathrm{O})(=\mathrm{O})) \mathrm{ccc} 1) \mathrm{c} 1$

$\mathrm{COc} 1 \mathrm{ccc} 2 \mathrm{C}=\mathrm{CC}(=\mathrm{O}) \mathrm{Oc} 2 \mathrm{c} 1$

$\mathrm{O}=\mathrm{C}(\mathrm{c}(\operatorname{ccc}(\mathrm{OC}) \mathrm{c} 1) \mathrm{c} 1) \mathrm{C}$

$\mathrm{O}=\mathrm{C}(\mathrm{O}) \mathrm{c}(\mathrm{c}(\mathrm{O}) \mathrm{ccc} 1 \mathrm{O}) \mathrm{c} 1$

$\mathrm{Nc}(\mathrm{c}(\mathrm{ccc} 1) \mathrm{CC}) \mathrm{c} 1$

c12ccnc1 cccc2OCC $(\mathrm{O}) \mathrm{CNC}(\mathrm{C}) \mathrm{C}$

1.66

$\begin{array}{lll}-0.05 & 1.61 \quad-0.10\end{array}$

$\begin{array}{lll}1.79 & 0.08 & 1.62\end{array}$

$\begin{array}{llll}2.46 & 0.75 & 1.80 & 0.09\end{array}$

$\begin{array}{llll}1.70 & -0.02 & 1.56 & -0.16\end{array}$

1.77

0.05

1.97

0.25

$-0.13$

1.87

0.07
0.15

0.15
-0.03

0.08

1.80

2.18

1.81

$-0.21$

$-0.15$

1.09

1.36

1.88

1.59

1.75

1.76

0.01

0.02

1.82

1.23

2.11

0.37

1.91

2.17

$-0.64$

$-0.38$

0.14

0.08

$-0.51$

0.17

$\mathrm{O}=\mathrm{C}(\mathrm{Nc}(\mathrm{c}(\mathrm{cc}(\mathrm{c}(\mathrm{ccc}(\mathrm{NC}) \mathrm{O}) \mathrm{CC}(=\mathrm{O}) \mathrm{C}$

1.75

c1C)c1)c2)C $) \mathrm{c} 2) \mathrm{CC}(=\mathrm{O}) \mathrm{C}$

$\mathrm{N}(\mathrm{CCCC} 1)(\mathrm{C} 1) \mathrm{CC}$

1.75

0.00

2.50

0.75

$\mathrm{O}=\mathrm{OC}(\mathrm{C})(\mathrm{C}) \mathrm{C}) \mathrm{C}$

1.89

0.14

2.11

0.36

$\mathrm{OC}(\mathrm{CCCC}) \mathrm{C}$

$\mathrm{NC}(\mathrm{C}) \mathrm{Cc}(\mathrm{cccc} 1) \mathrm{c} 1$

$\mathrm{O}=\mathrm{Cc}(\operatorname{ccc}(\mathrm{OC}) \mathrm{c} 1) \mathrm{c} 1$

$\mathrm{O}=\mathrm{C}(\mathrm{Nc}(\mathrm{c} 1 \mathrm{ccc} 2) \mathrm{c} 2) \mathrm{S} 1$

1.75

$-0.02$

$-0.01$

0.00

0.03

0.59

$-0.09$

0.10

$\mathrm{S}(\mathrm{SC}) \mathrm{C}$

$\mathrm{c}(\mathrm{c}(\mathrm{c}(\mathrm{N}) \mathrm{cc} 1) \mathrm{c}(\mathrm{N}) \mathrm{cc} 2)(\mathrm{c} 1) \mathrm{c} 2$

$\mathrm{O}=\mathrm{CCc}(\operatorname{cccc} 1) \mathrm{c} 1$

$\mathrm{O}=\mathrm{C}(\mathrm{OCC}(\mathrm{C}) \mathrm{C}) \mathrm{C}$

$\mathrm{O}=\mathrm{CCCCCC}$

$\mathrm{O}=\mathrm{C}(\mathrm{OCCCC}) \mathrm{C}$

0.07

1.84

0.06 
1,10-phenanthroline ethane, 1,1-dichlorophenol, 2-nitro-

1-propene, 3-bromo-

8-quinolinamine

1,3-benzenediol, 4-chloro-

benzoic acid, 3,4,5-trihydroxy-, propyl ester

pyrazine, 2-ethyl-3-methoxy-

pentanoic acid, 2-methyl-

ethane

pyrazine, 2-methyl-3-(methylthio)-

1-propanethiol

thiophene

benzaldehyde, 4-(dimethylamino)-

benzenesulfonic acid, 4-methyl-, ethyl ester

benzaldehyde, 2-hydroxy-

phenol, 4-ethoxy-

[1,1'-biphenyl]-4,4'-diamine, 3,3'-dimethoxy-

ethanone, 1-(2-methoxyphenyl)-

pyrazine, 2-ethoxy-3-methyl-

3,6-acridinediamine

benzoic acid, 3-nitro-

benzenamine, 4-chloro-

2-propanol, 1-[(1,1-dimethylethyl)amino]-3-[[4-(4-

morpholinyl)-1,2,5-thiadiazol-3-yl]oxy]-, 2-

butenedioate $(1: 1)$

4-thia-1-azabicyclo[3.2.0]heptane-2-carboxylic acid,

3,3-dimethyl-7-oxo-6-[(phenylacetyl)amino]-

benzeneacetic acid, methyl ester

5,12-naphthacenedione, 8-acetyl-10-[(3-amino-

2,3,6-trideoxy-alphal-hexopyranosyl)oxy]-7,8,9,10-

tetrahydro-6,8,11-trihydroxy-1-methoxy-

cyclohexanol, 2-methyl-

benzenamine, 2,6-dimethyl-

benzenepropanoic acid

imidazo[2,1-b]thiazole, 2,3,5,6-tetrahydro-6-phenylbenzene, nitro-

furan, 2-methyl-

benzenamine, 2-nitro-

ethanol, 2-(hexyloxy)-

3-pentanone, 2,4-dimethyl-

methane, bromotrifluoro-

benzoic acid, 4-amino-, ethyl ester

benzenepropanoic acid, -oxo-, ethyl ester

benzoic acid

2-propenoic acid, 2-methyl-, 1,2-ethanediylbis(oxy-

2,1-ethanediyl) ester

2-hexanone, 5-methyl-

2-propanol, 1-[4-(2-methoxyethyl)phenoxy]-3-[(1-

methylethyl)amino]-, 2,3-dihydroxybutanedioate

$(2: 1)$

benzenamine, 3-chloro-

methanone, phenyl-2-pyridinyl-

benzenepropanol

benzoic acid, 2-amino-, methyl ester

pyridine, 2,4,6-trimethyl-

benzoic acid, 4-nitro-

2,4,6-cycloheptatrien-1-one, 2-hydroxy-4-(1methylethyl)-

ethane, 1,1,2-trichloro-

2H-1-benzopyran-2-one, 7-hydroxy-4-methyl-

benzenamine, 2-chloro-

2-propenal, 3-phenyl-

propane, 2-chloro-

1,4-naphthalenediol

2,7-naphthalenediol

benzaldehyde, 2,5-dimethoxy- $\mathrm{n}(\mathrm{c}(\mathrm{c}(\mathrm{ccc} 1 \mathrm{cccn} 2) \mathrm{cc} 3) \mathrm{c} 12) \mathrm{c} 3$

$\mathrm{C}(\mathrm{Cl})(\mathrm{Cl}) \mathrm{C}$

$\mathrm{N}(=\mathrm{O})(=\mathrm{O}) \mathrm{c}(\mathrm{c}(\mathrm{O}) \mathrm{ccc} 1) \mathrm{c} 1$

$\mathrm{BrCC}=\mathrm{C}$

$\mathrm{n}(\mathrm{c}(\mathrm{c}(\mathrm{ccc} 1) \mathrm{cc} 2) \mathrm{c} 1 \mathrm{~N}) \mathrm{c} 2$

$\mathrm{Oc}(\mathrm{c}(\operatorname{ccc} 1 \mathrm{O}) \mathrm{Cl}) \mathrm{c} 1$

$\mathrm{O}=\mathrm{C}(\mathrm{OCCC}) \mathrm{c}(\mathrm{cc}(\mathrm{O}) \mathrm{c}(\mathrm{O}) \mathrm{c} 1 \mathrm{O}) \mathrm{c}$

$\mathrm{O}(\mathrm{c}(\mathrm{nccn} 1) \mathrm{c} 1 \mathrm{CC}) \mathrm{C}$

$\mathrm{O}=\mathrm{C}(\mathrm{O}) \mathrm{C}(\mathrm{CCC}) \mathrm{C}$

$\mathrm{CC}$

$\mathrm{n}(\mathrm{c}(\mathrm{SC}) \mathrm{c}(\mathrm{nc} 1) \mathrm{C}) \mathrm{c} 1$

SCCC

$\mathrm{S} 1 \mathrm{C}=\mathrm{CC}=\mathrm{C} 1$

$\mathrm{O}=\mathrm{Cc}(\operatorname{ccc}(\mathrm{N}(\mathrm{C}) \mathrm{C}) \mathrm{c} 1) \mathrm{c} 1$

$\mathrm{O}=\mathrm{S}(=\mathrm{O})(\mathrm{OCC}) \mathrm{c}(\operatorname{ccc}(\mathrm{c} 1) \mathrm{C}) \mathrm{c} 1$

$\mathrm{O}=\mathrm{Cc}(\mathrm{c}(\mathrm{O}) \mathrm{ccc} 1) \mathrm{c} 1$

$\mathrm{O}(\mathrm{c}(\mathrm{ccc}(\mathrm{O}) \mathrm{c} 1) \mathrm{c} 1) \mathrm{cC}$

$\mathrm{O}(\mathrm{c}(\mathrm{c}(\mathrm{N}) \operatorname{ccc} 1 \mathrm{c}(\operatorname{ccc}(\mathrm{N}) \mathrm{c} 2 \mathrm{OC}) \mathrm{c} 2) \mathrm{c} 1) \mathrm{C}$

$\mathrm{O}=\mathrm{C}(\mathrm{c}(\mathrm{c}(\mathrm{OC}) \operatorname{ccc} 1) \mathrm{c} 1) \mathrm{C}$

$\mathrm{O}(\mathrm{c}(\mathrm{nccn} 1) \mathrm{c} 1 \mathrm{C}) \mathrm{CC}$

$\mathrm{n}(\mathrm{c}(\mathrm{c}(\operatorname{ccc} 1 \mathrm{~N}) \operatorname{cc} 2 \mathrm{ccc}(\mathrm{N}) \mathrm{c} 3) \mathrm{c} 1) \mathrm{c} 23$

$\mathrm{O}=\mathrm{C}(\mathrm{O}) \mathrm{c}(\operatorname{cccc} 1 \mathrm{~N}(=\mathrm{O})(=\mathrm{O})) \mathrm{c} 1$

$\mathrm{Nc}(\operatorname{ccc}(\mathrm{c} 1) \mathrm{Cl}) \mathrm{c} 1$

$\mathrm{N} 1(\mathrm{C} 2=\mathrm{NSN}=\mathrm{C} 2 \mathrm{OCC}(\mathrm{O}) \mathrm{CNC}(\mathrm{C})$

(C)C)CCOCC1

$\mathrm{O}=\mathrm{C}(\mathrm{NC}(\mathrm{C}(=0) \mathrm{N} 1 \mathrm{C}(\mathrm{C}(=0) \mathrm{O}) \mathrm{C}(\mathrm{S} 2)$

(C)C)C12) Cc( $\operatorname{cccc} 3) \mathrm{c} 3$

$\mathrm{O}=\mathrm{C}(\mathrm{OC}) \mathrm{Cc}(\mathrm{cccc} 1) \mathrm{c} 1$

$\operatorname{coc} 4 \operatorname{cccc} 5 \mathrm{C}(=\mathrm{O}) \mathrm{c} 3 \mathrm{c}(\mathrm{O}) \mathrm{c} 2 \mathrm{Cc}(\mathrm{O})$

$(\mathrm{CC}(\mathrm{OC} 1 \mathrm{CC}(\mathrm{N}) \mathrm{C}(\mathrm{O}) \mathrm{C}(\mathrm{C}) \mathrm{O} 1) \mathrm{c} 2 \mathrm{c}(\mathrm{O}) \mathrm{c}$

$3 \mathrm{C}(=\mathrm{O}) \mathrm{c} 45) \mathrm{C}(\mathrm{C})=\mathrm{O}$

$\mathrm{OC}(\mathrm{C}(\mathrm{CCC} 1) \mathrm{C}) \mathrm{C} 1$

$\mathrm{Nc}(\mathrm{c}(\mathrm{ccc} 1) \mathrm{C}) \mathrm{c} 1 \mathrm{C}$

$\mathrm{O}=\mathrm{C}(\mathrm{O}) \mathrm{CCc}(\operatorname{cccc} 1) \mathrm{c} 1$

$\mathrm{N} 3=\mathrm{C} 2 \mathrm{~N}(\mathrm{CC} 3 \mathrm{c} 1 \mathrm{ccccc} 1) \mathrm{CCS} 2$

$\mathrm{N}(=\mathrm{O})(=\mathrm{O}) \mathrm{c}(\operatorname{cccc} 1) \mathrm{c} 1$

$\mathrm{C} 1=\mathrm{C}(\mathrm{C}) \mathrm{OC}=\mathrm{C} 1$

$\mathrm{N}(=\mathrm{O})(=\mathrm{O}) \mathrm{c}(\mathrm{c}(\mathrm{N}) \mathrm{ccc} 1) \mathrm{c} 1$

$\mathrm{O}(\mathrm{CCCCCC}) \mathrm{CCO}$

$\mathrm{O}=\mathrm{C}(\mathrm{C}(\mathrm{C}) \mathrm{C}) \mathrm{C}(\mathrm{C}) \mathrm{C}$

$\mathrm{FC}(\mathrm{F})(\mathrm{F}) \mathrm{Br}$

$\mathrm{O}=\mathrm{C}(\mathrm{OCC}) \mathrm{c}(\operatorname{ccc}(\mathrm{N}) \mathrm{c} 1) \mathrm{c} 1$

$\mathrm{O}=\mathrm{C}(\mathrm{OCC}) \mathrm{CC}(=\mathrm{O}) \mathrm{c}(\operatorname{cccc} 1) \mathrm{c} 1$

$\mathrm{O}=\mathrm{C}(\mathrm{O}) \mathrm{c}(\mathrm{cccc} 1) \mathrm{c} 1$

$\mathrm{O}=\mathrm{C}(\mathrm{OCCOC \operatorname {Coccoc }}(=\mathrm{O}) \mathrm{C}(=\mathrm{C}) \mathrm{C})$

$\mathrm{C}(=\mathrm{C}) \mathrm{C}$

$\mathrm{O}=\mathrm{C}(\mathrm{CcC}(\mathrm{C}) \mathrm{C}) \mathrm{C}$

$\mathrm{CC}(\mathrm{C}) \mathrm{NCC}(\mathrm{O}) \mathrm{COc} 1 \mathrm{ccc}(\mathrm{CCOC}) \mathrm{cc} 1$

$\mathrm{Nc}(\operatorname{cccc} 1 \mathrm{Cl}) \mathrm{c} 1$

$\mathrm{O}=\mathrm{C}(\mathrm{c}(\mathrm{cccc} 1) \mathrm{c} 1) \mathrm{c}(\mathrm{nccc} 2) \mathrm{c} 2$

OCCCc( $\operatorname{cccc} 1) \mathrm{c} 1$

$\mathrm{O}=\mathrm{C}(\mathrm{OC}) \mathrm{c}(\mathrm{c}(\mathrm{N}) \operatorname{ccc} 1) \mathrm{c} 1$

$\mathrm{n}(\mathrm{c}(\mathrm{cc}(\mathrm{c} 1) \mathrm{C}) \mathrm{C}) \mathrm{c} 1 \mathrm{C}$

$\mathrm{O}=\mathrm{C}(\mathrm{O}) \mathrm{c}(\operatorname{ccc}(\mathrm{N}(=\mathrm{O})(=\mathrm{O})) \mathrm{c} 1) \mathrm{c} 1$

$\mathrm{O}=\mathrm{C} 1 \mathrm{C}=\mathrm{CC}=\mathrm{C}(\mathrm{C}(\mathrm{C}) \mathrm{C}) \mathrm{C}=\mathrm{C} 1 \mathrm{O}$

$\mathrm{ClCC}(\mathrm{Cl}) \mathrm{Cl}$

$\mathrm{c} 1(\mathrm{O}) \mathrm{cc} 2 \mathrm{OC}(=\mathrm{O}) \mathrm{C}=\mathrm{C}(\mathrm{C}) \mathrm{c} 2 \mathrm{cc} 1$

$\mathrm{Nc}(\mathrm{c}(\mathrm{ccc} 1) \mathrm{Cl}) \mathrm{c} 1$

$\mathrm{O}=\mathrm{CC}=\mathrm{Cc}(\operatorname{cccc} 1) \mathrm{c} 1$

$\mathrm{C}(\mathrm{Cl})(\mathrm{C}) \mathrm{C}$

Oc(c(c(c(O)c1)ccc2)c2)c1

Oc( $\operatorname{ccc}(\mathrm{c} 1 \mathrm{cc}(\mathrm{O}) \mathrm{c} 2) \mathrm{c} 2) \mathrm{c} 1$

$\mathrm{O}=\mathrm{Cc}(\mathrm{c}(\mathrm{OC}) \operatorname{ccc} 1 \mathrm{OC}) \mathrm{c} 1$

1.78

1.79

1.79

1.79

1.79

1.80

1.80

1.80

1.80

1.81

1.81

1.81

1.81

1.81

1.81

1.81

1.81

1.81

1.82

1.82

1.83

1.83

1.83

1.83

2.29

0.51

2.31

0.53

1.91

$-0.03$

1.72
1.91

$-0.07$

2.02

2.03

0.23

0.24

$-0.12$

$-0.01$

0.15

0.18

$-0.49$

$-0.18$

$-0.05$

0.00

0.08

0.15

0.20

0.27

0.27

$-0.07$

0.13

$-0.35$

$-0.14$

$-0.11$

$-0.08$

1.98

1.51

1.54

1.84

1.64

1.79

1.44

1.42

1.72

1.89

1.80

1.77

1.22

1.92

2.02

1.71

1.64

2.10

1.52

1.95

1.44

0.19

$-0.28$

$-0.26$

0.04

$-0.16$

$-0.01$

$-0.37$

$-0.39$

$-0.09$

0.08

$-0.01$

$-0.04$

$-0.59$

0.11

0.21

$-0.11$

$-0.18$

0.27

$-0.31$

0.12

$-0.39$

1.83

1.85

0.02

1.92

0.09

0.25

2.03

0.20

$1.83 \quad 2.19$

0.36

1.68

$-0.15$

0.21

1.80

2.05

2.17

2.29

2.87

1.81

1.91

2.02

1.55

1.58

1.59

1.80

1.71

1.87

1.66

0.33

1.80
1.77

$-0.04$

0.45

1.03

$-0.04$

0.06

0.17

$-0.31$

$-0.28$

$-0.27$

$-0.06$

$-0.16$

0.00

$-0.22$

1.66

$-0.22$

1.84

2.20

1.89

1.75

1.43

1.82

1.91

1.55

2.20

1.71

1.72

1.58

1.88

$-0.19$

1.80

$-0.08$

1.88

1.88

1.88

1.88

1.88 
phenol, 4-nitro-

pregna-1,4-diene-3,20-dione, 16,21-bis(acetyloxy)9-fluoro-11,17-dihydroxy-, (11-beta,16-alpha-)-

benzene, (methoxymethyl)-

1,4-naphthalenedione, 5-hydroxy-

ethanone, 1-(2-hydroxyphenyl)-

hexanoic acid

butane, 1,1-[oxybis(2,1-ethanediyloxy)]bis-

strychnidin-10-one

acetic acid, 2-methylphenyl ester pregna-1,4-diene-3,20-dione, 9-fluoro-11,17,21trihydroxy-16-methyl-, (11-beta,16-beta)-

2-propenoic acid, 2-methyl-, ethyl ester phenol, 4-methyl-

benzenamine, 4-methoxy-2-nitro-

1,5-naphthalenediol

1,7-naphthalenediol

2-propen-1-ol, 3-phenyl-

ethane, 1,1-thiobis-

phenol, 2-methyl-

2-propenenitrile, 3-phenyl-

benzoic acid, 4-methoxy-

2,2:6 2-terpyridine

benzoic acid, 4-hydroxy-, methyl ester

ethane, 1,2-dibromo-

phenol, 3-methyl-

acetic acid, phenylmethyl ester

benzenamine, 4-ethyl-

cyclohexanecarboxylic acid

pyrazine, 2-methyl-3-(2-methylpropyl)-

methane, trichloro-

benzeneethanol, alpha-methyl-

1,3-naphthalenediol

2(1H)-naphthalenone, octahydro-

propane, 1,1,1-triethoxy-

7-quinolinol

2-heptanone

benzenemethanamine, N,N-dimethyl-

2H-1-benzopyran-2-one, 4-hydroxy-3-[1-(4-

nitrophenyl)-3-oxobutyl]-

phenol, 3-ethoxy-

benzene, 1-methyl-2,4-dinitro-

propane, 1,2-dichloro-

ethanol, 2-(4-chlorophenoxy)-

1,3-butadiene

benzene, 1,1-[sulfinylbis(methylene)]bis-

1,4-benzenedicarboxylic acid

phenol, 3-nitro-

pregna-1,4-diene-3,20-dione, 9-fluoro-11,17-

dihydroxy-6-methyl-, (6-alpha,11)-

ethane, iodo-

ethane, hexafluoro-

benzene, nitroso-

benzothiazole

8-quinolinol

benzoic acid, 3-methoxy-

cholan-24-oic acid, 3,7,12-trihydroxy-, (3-alpha,5 ,

7-alpha,12-alpha)-

1H-indazole, 5-nitro-

1-hexanol

2-propanol, 1,1,1-trichloro-2-methyl-

quinoline

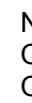

$\mathrm{N}(=\mathrm{O})(=\mathrm{O}) \mathrm{c}(\operatorname{ccc}(\mathrm{O}) \mathrm{c} 1) \mathrm{c} 1$
$\mathrm{O}=\mathrm{C} 1 \mathrm{C}=\mathrm{C} 2 \mathrm{CCC} 3 \mathrm{C} 4 \mathrm{CC}(\mathrm{OC}(=\mathrm{O}) \mathrm{C}) \mathrm{C}($
$\mathrm{O})$
$(\mathrm{C}(=\mathrm{O}) \mathrm{COC}(=\mathrm{O}) \mathrm{C}) \mathrm{C} 4(\mathrm{C}) \mathrm{CC}(\mathrm{O}) \mathrm{C} 3(\mathrm{~F})$
$\mathrm{C} 2(\mathrm{C}) \mathrm{C}=\mathrm{C} 1$

$\mathrm{O}(\mathrm{Cc}(\operatorname{coc} 1) \mathrm{c} 1) \mathrm{C}$

$\operatorname{Oc} 1 \operatorname{cccc} 2 \mathrm{C}(=\mathrm{O}) \mathrm{C}=\mathrm{CC}(=\mathrm{O}) \mathrm{c} 12$

$\mathrm{O}=\mathrm{C}(\mathrm{c}(\mathrm{c}(\mathrm{O}) \mathrm{ccc} 1) \mathrm{c} 1) \mathrm{C}$

$\mathrm{O}=\mathrm{C}(\mathrm{O}) \mathrm{CCCCC}$

$\mathrm{O}(\mathrm{CCOCCOCCCC}) \mathrm{CCCC}$

$\mathrm{O}=\mathrm{C}(\mathrm{N}(\mathrm{c}(\mathrm{c}(\mathrm{C} 1(\mathrm{C}(\mathrm{N}) \mathrm{C} 2) \mathrm{CC}(\mathrm{C} 3 \mathrm{C} 4 \mathrm{C} 5$

$\mathrm{OC} 6)=\mathrm{C} 6) \mathrm{C} 3) \mathrm{C} 2) \mathrm{ccc} 7) \mathrm{c} 7) \mathrm{C} 14) \mathrm{C} 5$

$\mathrm{O}=\mathrm{C}(\mathrm{Oc}(\mathrm{c}(\operatorname{ccc} 1) \mathrm{C}) \mathrm{c} 1) \mathrm{C}$

$\mathrm{C} 1=\mathrm{CC}(=\mathrm{O}) \mathrm{C}=\mathrm{C} 2 \mathrm{CCC} 3 \mathrm{C} 4 \mathrm{CC}(\mathrm{C}) \mathrm{C}(\mathrm{O}$

1.91

1.91

0.00

1.93

1.51

$-0.41$

2.05

0.02

)$(\mathrm{C}(=\mathrm{O}) \mathrm{CO}) \mathrm{C} 4(\mathrm{C}) \mathrm{CC}(\mathrm{O}) \mathrm{C} 3(\mathrm{~F}) \mathrm{C} 12 \mathrm{C}$

$\mathrm{O}=\mathrm{C}(\mathrm{OCC}) \mathrm{C}(=\mathrm{C}) \mathrm{C}$

$\mathrm{Oc}(\operatorname{ccc}(\mathrm{c} 1) \mathrm{C}) \mathrm{c} 1$

$\mathrm{N}(=\mathrm{O})(=\mathrm{O}) \mathrm{c}(\mathrm{c}(\mathrm{N}) \mathrm{ccc} 1 \mathrm{OC}) \mathrm{c} 1$

$\mathrm{Oc}(\mathrm{c}(\mathrm{c}(\mathrm{c}(\mathrm{O}) \mathrm{cc} 1) \mathrm{cc} 2) \mathrm{c} 1) \mathrm{c} 2$

$\mathrm{Oc} 1 \mathrm{ccc} 2 \mathrm{cccc}(\mathrm{O}) \mathrm{c} 2 \mathrm{c} 1$

$\mathrm{OCC}=\mathrm{Cc}(\operatorname{cccc} 1) \mathrm{c} 1$

$\mathrm{S}(\mathrm{CC}) \mathrm{CC}$

Oc(c(ccc1)C $) \mathrm{c} 1$

$\mathrm{C}(\# \mathrm{~N}) \mathrm{C}=\mathrm{Cc}(\mathrm{cccc} 1) \mathrm{c} 1$

$\mathrm{O}=\mathrm{C}(\mathrm{O}) \mathrm{c}(\operatorname{ccc}(\mathrm{OC}) \mathrm{c} 1) \mathrm{c} 1$

$\mathrm{n}(\mathrm{c}(\mathrm{ccc} 1) \mathrm{c}(\mathrm{nc}(\mathrm{c}(\mathrm{nccc} 2) \mathrm{c} 2) \mathrm{cc} 3) \mathrm{c} 3) \mathrm{c} 1$

$\mathrm{O}=\mathrm{C}(\mathrm{OC}) \mathrm{c}(\operatorname{ccc}(\mathrm{O}) \mathrm{c} 1) \mathrm{c} 1$

$\mathrm{BrCCBr}$

$\mathrm{Oc}(\operatorname{cccc} 1 \mathrm{C}) \mathrm{c} 1$

$\mathrm{O}=\mathrm{C}(\mathrm{OCc}(\operatorname{cccc} 1) \mathrm{c} 1) \mathrm{C}$

$\mathrm{Nc}(\mathrm{ccc}(\mathrm{c} 1) \mathrm{CC}) \mathrm{c} 1$

$\mathrm{O}=\mathrm{C}(\mathrm{O}) \mathrm{C}(\mathrm{CCCC} 1) \mathrm{C} 1$

$\mathrm{n}(\mathrm{c}(\mathrm{c}(\mathrm{nc} 1) \mathrm{CC}(\mathrm{C}) \mathrm{C}) \mathrm{C}) \mathrm{c} 1$

$\mathrm{C}(\mathrm{Cl})(\mathrm{Cl}) \mathrm{Cl}$

$\mathrm{OC}(\mathrm{C}) \mathrm{Cc}(\mathrm{cccc} 1) \mathrm{c} 1$

Oc(c(c(ccc1)cc2O)c1)c2

$\mathrm{O}=\mathrm{C}(\mathrm{CCC}(\mathrm{C} 1 \mathrm{CCC} 2) \mathrm{C} 2) \mathrm{C} 1$

$\mathrm{O}(\mathrm{C}(\mathrm{OCC})(\mathrm{OCC}) \mathrm{CC}) \mathrm{CC}$

$\mathrm{n}(\mathrm{c}(\mathrm{c}(\mathrm{ccc} 10) \mathrm{cc} 2) \mathrm{c} 1) \mathrm{c} 2$

$\mathrm{O}=\mathrm{C}(\mathrm{CCCCC}) \mathrm{C}$

$\mathrm{N}(\mathrm{Cc}(\operatorname{cccc} 1) \mathrm{c} 1)(\mathrm{C}) \mathrm{C}$

$\mathrm{O}=\mathrm{C} 1 \mathrm{Oc} 2 \operatorname{ccccc} 2 \mathrm{C}(\mathrm{O})=\mathrm{C} 1 \mathrm{C}(\mathrm{CC}(=\mathrm{O})$

C) $\operatorname{cocc}(\mathrm{N}(=\mathrm{O})=\mathrm{O}) \operatorname{cc} 3$

$\mathrm{O}(\mathrm{c}(\operatorname{cccc} 1 \mathrm{O}) \mathrm{c} 1) \mathrm{CC}$

$\mathrm{N}(=\mathrm{O})(=\mathrm{O}) \mathrm{c}(\operatorname{ccc}(\operatorname{c} 1 \mathrm{~N}(=\mathrm{O})(=\mathrm{O})) \mathrm{C}) \mathrm{c} 1$

$\mathrm{ClCC}(\mathrm{Cl}) \mathrm{C}$

$\mathrm{O}(\mathrm{c}(\mathrm{ccc}(\mathrm{c} 1) \mathrm{Cl}) \mathrm{c} 1) \mathrm{CCO}$

$\mathrm{C}(\mathrm{C}=\mathrm{C})=\mathrm{C}$

$\mathrm{O}=\mathrm{S}(\mathrm{Cc}(\mathrm{cccc} 1) \mathrm{c} 1) \mathrm{Cc}(\mathrm{cccc} 2) \mathrm{c} 2$

$\mathrm{O}=\mathrm{C}(\mathrm{O}) \mathrm{c}(\operatorname{ccc}(\mathrm{c} 1) \mathrm{C}(=\mathrm{O}) \mathrm{O}) \mathrm{c} 1$

$\mathrm{N}(=\mathrm{O})(=\mathrm{O}) \mathrm{c}(\operatorname{cccc} 1 \mathrm{O}) \mathrm{c} 1$

$\mathrm{CC} 1 \mathrm{CC} 2 \mathrm{C} 3 \mathrm{CCC}(\mathrm{O})$

$(\mathrm{C}(=\mathrm{O}) \mathrm{C}) \mathrm{C} 3(\mathrm{C}) \mathrm{CC}(\mathrm{O}) \mathrm{C} 2(\mathrm{~F}) \mathrm{C} 4(\mathrm{C}) \mathrm{C}=$ $\mathrm{CC}(=\mathrm{O}) \mathrm{C}=\mathrm{C} 14$

$\mathrm{C}(\mathrm{C}) \mathrm{I}$

$\mathrm{FC}(\mathrm{F})(\mathrm{F}) \mathrm{C}(\mathrm{F})(\mathrm{F}) \mathrm{F}$

$\mathrm{O}=\mathrm{Nc}(\operatorname{cccc} 1) \mathrm{c} 1$

c1ccc2ncsc2c1

$\mathrm{n}(\mathrm{c}(\mathrm{c}(\mathrm{ccc} 1) \mathrm{cc} 2) \mathrm{c} 1 \mathrm{O}) \mathrm{c} 2$

$\mathrm{O}=\mathrm{C}(\mathrm{O}) \mathrm{c}(\operatorname{cccc} 1 \mathrm{OC}) \mathrm{c} 1$

$\mathrm{O}=\mathrm{C}(\mathrm{O}) \mathrm{CCC}(\mathrm{C}(\mathrm{C}(\mathrm{C}(\mathrm{C}(\mathrm{C}(\mathrm{C}) \mathrm{C}(\mathrm{C} 1) \mathrm{CC}$

$(\mathrm{O}) \mathrm{C} 2)(\mathrm{C} 2) \mathrm{C}) \mathrm{C} 3) \mathrm{C} 1 \mathrm{O}) \mathrm{C} 4)$

$(\mathrm{C} 3 \mathrm{O}) \mathrm{C}) \mathrm{C} 4) \mathrm{C}$

$\mathrm{O}=\mathrm{N}(=\mathrm{O}) \mathrm{c}(\operatorname{ccc}(\mathrm{NN}=\mathrm{C} 1) \mathrm{c} 12) \mathrm{c} 2$

OCCCCCC

$\mathrm{OC}(\mathrm{C}(\mathrm{Cl})(\mathrm{Cl}) \mathrm{Cl})(\mathrm{C}) \mathrm{C}$

$\mathrm{n}(\mathrm{c}(\mathrm{c}(\mathrm{ccc} 1) \mathrm{cc} 2) \mathrm{c} 1) \mathrm{c} 2$

2.03

2.03

2.03

1.77

1.96

1.97

2.05

2.46

1.85

2.14

1.72

1.77

2.06

2.10

2.21

2.21

1.84

1.90

2.06

1.84

1.96

1.96

2.00

2.01

2.06

2.08

2.11

2.36

2.43

1.52

1.98

2.21

2.49

3.10

1.66

1.73

1.75

2.05

2.08

2.18

2.25

1.74

2.03

2.19

1.76

1.91

2.06

$-0.15$

0.04

0.05

0.13

0.54

$-0.08$

0.21

$-0.22$

$-0.17$

0.12

0.16

0.27

0.27

$-0.11$

$-0.05$

0.11

$-0.12$

0.00

0.00

0.04

0.05

0.10

0.12

0.15

0.40

0.47

$-0.45$

0.01

0.24

0.52

1.13

$-0.32$

$-0.25$

$-0.23$

0.07

0.10

0.20

0.27

$-0.25$

0.04

0.20

$-0.24$

$-0.09$

0.06

1.69

1.93

1.57

$\begin{array}{ll}1.88 & -0.04\end{array}$

$2.48 \quad 0.56$

\begin{tabular}{ll}
$1.68-0.25$ \\
\hline
\end{tabular}

$\begin{array}{ll}1.96 & 0.03\end{array}$

$1.93-0.01$

$1.69-0.25$

$\begin{array}{ll}1.95 & 0.01\end{array}$

$1.61-0.33$

$\begin{array}{ll}1.96 & 0.02\end{array}$

$2.00 \quad 0.06$

$1.93-0.02$

$2.46 \quad 0.51$

$1.89-0.06$

$2.01 \quad 0.05$

$1.63-0.33$

$\begin{array}{ll}2.78 & 0.82\end{array}$

$2.17 \quad 0.21$

$\begin{array}{ll}2.08 & 0.12\end{array}$

$1.93-0.03$

$2.07 \quad 0.11$

$1.91-0.05$

$\begin{array}{ll}1.88 & -0.08\end{array}$

$2.04 \quad 0.08$

$1.67-0.30$

$1.95-0.02$

$\begin{array}{ll}2.02 & 0.05\end{array}$

$2.85 \quad 0.88$

$1.89-0.08$

$1.93-0.05$

$1.92-0.06$

$1.84-0.14$

$2.53 \quad 0.55$

$1.88-0.10$

$1.90-0.08$

$2.13 \quad 0.15$

$1.91-0.08$

$1.94-0.05$

$\begin{array}{ll}2.28 & 0.29\end{array}$

$1.01-0.99$

$\begin{array}{ll}1.01 & -0.99 \\ 1.92 & -0.08\end{array}$

$2.34 \quad 0.34$

\subsection{8}

2.15

1.86

2.17

1.66

1.96

0.08

2.29

2.46

$-0.15 \quad 1.41$

$0.16 \quad 2.13$

$-0.36$

$-0.06$

1.91

1.61

2.26

0.29

0.46

$-0.60$

0.12

$-0.11$ 
benzoic acid, 5-(aminosulfonyl)-4-chloro-2-[(2-

furanylmethyl)amino]-

quinoxaline, 5-methyl-

benzoic acid, 2-methoxy-, methyl ester

propane, 1-chloro-

benzene, 1,4-dimethoxy-

2H-1,4-benzodiazepin-2-one, 7-bromo-1,3-dihydro-

5-(2-pyridinyl)-

benzoic acid, 2-chloro-

1-hexanamine

benzeneethanamine, $\mathrm{N}$-alpha-dimethyl-

isoquinoline

7-oxabicyclo[4.1.0]heptane, 3-ethenyl-

acetonitrile, trichloro-

methane, trichloronitro-

acetamide, $\mathrm{N}$-(4-chlorophenyl)-

ethene, 1,2-dichloro-, (E)-

acetic acid, 3-methylphenyl ester

phenol, 2-(1-methylethoxy)-

ethanol, 2-(ethylphenylamino)-

2-propanol, 1-[(1-methylethyl)amino]-3-[2-(2-

propenyloxy)phenoxy]-

2,4,6(1H,3H,5H)-pyrimidinetrione, 5-ethyl-5-(1methylbutyl)-

pregn-4-ene-3,11,20-trione, 21-(acetyloxy)-17-

hydroxy-

propane, 1-bromo-

benzene, 2-methyl-1,3-dinitro-

ethanone, 1-(4-methylphenyl)-

2,4(1H,3H)-pyrimidinedione, 5-bromo-6-methyl-3-

(1-methylpropyl)-

benzene, (2-nitroethenyl)-

benzene, methoxy-

acetic acid, 4-methylphenyl ester

benzoic acid, methyl ester

phenol, 2-methyl-4,6-dinitro-

4H-[1,2,4]triazolo[4,3-a][1,4]benzodiazepine, 8chloro-1-methyl-6-phenyl-

ethanone, 2-hydroxy-1,2-diphenyl-

benzene

2-propenoic acid, 3-phenyl-

ethene, 1,1-dichloro-

$1 \mathrm{H}$-indole

propane, 2-bromo-

phenol, 2-chloro-

benzenamine, $\mathrm{N}, 4$-dimethyl-

methane, dichlorodifluoro-

threo-alpha-galacto-octopyranoside, methyl 7-

chloro-6,7,8-trideoxy-6-[[[1-methyl-4-propyl-2-

pyrrolidinyl]carbonyl]amino]-1-thio-

benzene, (ethoxymethyl)-

benzene, 1-chloro-2,4-dinitro-

spiro[benzofuran-2(3H), 1'-[2]cyclohexene]-3,4'-

dione, 7-chloro-2',4,6-trimethoxy-6'-beta-methyl-

3-buten-2-one, 4-phenyl-

benzamide, N,N-diethyl-3-methyl-

propane, 1-bromo-3-chloro-

cyclohexanone, 2-(2-chlorophenyl)-2-

(methylamino)-

2H-1,4-benzodiazepin-2-one, 7-chloro-1,3-dihydro3-hydroxy-1-methyl-5-phenyl-

1-propanone, 1-phenyl-

pregn-4-ene-3,20-dione, 21-(acetyloxy)-11,17-

dihydroxy-, (11)-

1,4-naphthalenedione, 2-methyl-

\begin{tabular}{|c|c|c|c|c|c|}
\hline $\begin{array}{l}\mathrm{NS}(=\mathrm{O})(=\mathrm{O}) \mathrm{c} 2 \mathrm{cc}(\mathrm{C}(=\mathrm{O}) \\
(\mathrm{O}) \mathrm{c}(\mathrm{NCc} 1 \mathrm{ccco} 1) \mathrm{cc} 2 \mathrm{Cl}\end{array}$ & 2.03 & 2.32 & 0.29 & 2.71 & 0.68 \\
\hline $\mathrm{n}(\mathrm{c}(\mathrm{c}(\mathrm{nc} 1) \mathrm{ccc} 2) \mathrm{c} 2 \mathrm{C}) \mathrm{c} 1$ & 2.04 & 1.66 & -0.38 & 1.54 & -0.50 \\
\hline $\mathrm{O}=\mathrm{C}(\mathrm{OC}) \mathrm{c}(\mathrm{c}(\mathrm{OC}) \mathrm{ccc} 1) \mathrm{c} 1$ & 2.04 & 1.91 & -0.13 & 2.06 & 0.02 \\
\hline $\mathrm{CICCC}$ & 2.04 & 2.07 & 0.03 & 2.09 & 0.05 \\
\hline $\mathrm{O}(\mathrm{c}(\mathrm{ccc}(\mathrm{OC}) \mathrm{c} 1) \mathrm{c} 1) \mathrm{C}$ & 2.04 & 2.15 & 0.11 & 2.05 & 0.01 \\
\hline $\begin{array}{l}\mathrm{c} 1 \mathrm{c}(\mathrm{Br}) \operatorname{cc} 2 \mathrm{C}(\mathrm{c} 3 \mathrm{nccc} 3)=\mathrm{NCC}(=\mathrm{O}) \mathrm{Nc} \\
2 \mathrm{c} 1\end{array}$ & 2.05 & 1.93 & -0.12 & 2.09 & 0.04 \\
\hline $\mathrm{O}=\mathrm{C}(\mathrm{O}) \mathrm{c}(\mathrm{c}(\mathrm{ccc} 1) \mathrm{Cl}) \mathrm{c} 1$ & 2.05 & 2.18 & 0.13 & 2.39 & 0.34 \\
\hline NCCCCCC & 2.06 & 1.82 & -0.24 & 1.98 & -0.08 \\
\hline $\mathrm{CNC}(\mathrm{C}) \mathrm{Cc} 1 \mathrm{ccccc} 1$ & 2.07 & 2.22 & 0.15 & 2.23 & 0.16 \\
\hline $\mathrm{n}(\operatorname{ccc}(\mathrm{c} 1 \mathrm{ccc} 2) \mathrm{c} 2) \mathrm{c} 1$ & 2.08 & 2.14 & 0.06 & 2.14 & 0.06 \\
\hline $\mathrm{O}(\mathrm{C} 1 \mathrm{CC}(\mathrm{C}=\mathrm{C}) \mathrm{C} 22) \mathrm{C} 12$ & 2.08 & 2.43 & 0.35 & 1.65 & -0.43 \\
\hline $\mathrm{C}(\# \mathrm{~N}) \mathrm{C}(\mathrm{Cl})(\mathrm{Cl}) \mathrm{Cl}$ & 2.09 & 1.21 & -0.88 & 1.80 & -0.29 \\
\hline $\mathrm{N}(=\mathrm{O})(=\mathrm{O}) \mathrm{C}(\mathrm{Cl})(\mathrm{Cl}) \mathrm{Cl}$ & 2.09 & 1.32 & -0.77 & 2.10 & 0.01 \\
\hline $\mathrm{O}=\mathrm{C}(\mathrm{Nc}(\mathrm{ccc}(\mathrm{c} 1) \mathrm{Cl}) \mathrm{c} 1) \mathrm{C}$ & 2.09 & 1.74 & -0.35 & 1.72 & -0.37 \\
\hline $\mathrm{C}(=\mathrm{CCl}) \mathrm{Cl}$ & 2.09 & 1.98 & -0.11 & 1.85 & -0.24 \\
\hline $\mathrm{O}=\mathrm{C}(\mathrm{Oc}(\operatorname{ccc} 1 \mathrm{C}) \mathrm{c} 1) \mathrm{C}$ & 2.09 & 2.14 & 0.05 & 1.95 & -0.14 \\
\hline $\mathrm{O}(\mathrm{c}(\mathrm{c}(\mathrm{O}) \mathrm{ccc} 1) \mathrm{c} 1) \mathrm{C}(\mathrm{C}) \mathrm{C}$ & 2.09 & 2.25 & 0.16 & 2.27 & 0.18 \\
\hline $\operatorname{OCCN}(\mathrm{c}(\operatorname{cccc} 1) \mathrm{c} 1) \mathrm{CC}$ & 2.10 & 1.69 & -0.41 & 1.72 & -0.38 \\
\hline $\mathrm{CC}(\mathrm{C}) \mathrm{NCC}(\mathrm{O}) \mathrm{COc} 1 \mathrm{c}(\mathrm{OCC}=\mathrm{C}) \mathrm{cccc} 1$ & 2.10 & 1.83 & -0.27 & 2.44 & 0.34 \\
\hline $\begin{array}{l}\operatorname{Cccc}(\mathrm{C}) \mathrm{C} 1(\mathrm{CC}) \mathrm{C}(=\mathrm{O}) \mathrm{NC}(=\mathrm{O}) \mathrm{NC} 1= \\
\mathrm{O}\end{array}$ & 2.10 & 2.00 & -0.10 & 2.16 & 0.06 \\
\hline $\begin{array}{l}\mathrm{CC}(=\mathrm{O}) \mathrm{OCC}(=\mathrm{O}) \mathrm{C} 3(\mathrm{O}) \mathrm{CCC} 4 \mathrm{C} 2 \mathrm{CCC} \\
1=\mathrm{CC}(=\mathrm{O}) \mathrm{CCC} 1(\mathrm{C}) \mathrm{C} 2 \mathrm{C}(=\mathrm{O}) \mathrm{CC} 34 \mathrm{C}\end{array}$ & 2.10 & 2.14 & 0.04 & 2.35 & 0.25 \\
\hline BrCCC & 2.10 & 2.16 & 0.06 & 2.18 & \\
\hline $\mathrm{N}(=\mathrm{O})(=0) \mathrm{c}(\mathrm{c}(\mathrm{c}(\mathrm{N}(=\mathrm{O})(=0)) \mathrm{cc} 1) \mathrm{C}) \mathrm{c} 1$ & 2.10 & 2.18 & 0.08 & 1.81 & -0.29 \\
\hline $\mathrm{O}=\mathrm{C}(\mathrm{c}(\operatorname{ccc}(\mathrm{c} 1) \mathrm{C}) \mathrm{c} 1) \mathrm{C}$ & 2.10 & 2.22 & 0.12 & 2.11 & 0.01 \\
\hline $\begin{array}{l}\mathrm{N} 1 \mathrm{C}(=\mathrm{O}) \mathrm{N}(\mathrm{C}(\mathrm{C}) \mathrm{CC}) \mathrm{C}(=\mathrm{O}) \mathrm{C}(\mathrm{Br})=\mathrm{C} 1 \\
\mathrm{C}\end{array}$ & 2.11 & 1.68 & -0.43 & 1.20 & \\
\hline $\mathrm{N}(=\mathrm{O})(=\mathrm{O}) \mathrm{C}=\mathrm{Cc}(\operatorname{ccc} 1) \mathrm{c} 1$ & 2.11 & 1.95 & -0.16 & 2.17 & \\
\hline $\mathrm{O}(\mathrm{c}(\operatorname{cccc} 1) \mathrm{c} 1) \mathrm{C}$ & 2.11 & 2.07 & -0.04 & 2.10 & -0.01 \\
\hline $\mathrm{O}=\mathrm{C}(\mathrm{Oc}(\mathrm{ccc}(\mathrm{c} 1) \mathrm{C}) \mathrm{c} 1) \mathrm{C}$ & 2.11 & 2.14 & 0.03 & 1.96 & -0.1 \\
\hline $\mathrm{O}=\mathrm{C}(\mathrm{OC}) \mathrm{c}(\operatorname{cccc} 1) \mathrm{c} 1$ & 2.12 & 1.83 & -0.29 & 1.98 & -0.14 \\
\hline $\begin{array}{l}\mathrm{N}(=\mathrm{O})(=\mathrm{O}) \mathrm{c}(\mathrm{cc}(\mathrm{N}(=\mathrm{O}) \\
(=\mathrm{O})) \mathrm{c}(\mathrm{O}) \mathrm{c} 1 \mathrm{C}) \mathrm{c} 1\end{array}$ & 2.12 & 2.27 & 0.15 & 2.09 & \\
\hline $\begin{array}{l}\mathrm{c} 1 \mathrm{cc} 2 \mathrm{n} 3 \mathrm{c}(\mathrm{C}) \mathrm{nnc} 3 \mathrm{CN}=\mathrm{C}(\mathrm{c} 4 \mathrm{ccccc} 4) \mathrm{c} 2 \\
\mathrm{cc} 1 \mathrm{Cl}\end{array}$ & 2.12 & 3.87 & 1.75 & 2.23 & 0.11 \\
\hline $\mathrm{O}=\mathrm{C}(\mathrm{c}(\operatorname{cccc} 1) \mathrm{c} 1) \mathrm{C}(\mathrm{O}) \mathrm{c}(\operatorname{cccc} 2) \mathrm{c} 2$ & 2.13 & 1.84 & -0.29 & 2.64 & 0.5 \\
\hline$c(\operatorname{cccc} 1) c 1$ & 2.13 & 1.99 & -0.14 & 2.03 & 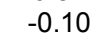 \\
\hline $\mathrm{O}=\mathrm{C}(\mathrm{O}) \mathrm{C}=\mathrm{Cc}(\operatorname{ccc} 1) \mathrm{c} 1$ & 2.13 & 2.07 & -0.06 & 2.38 & 0.25 \\
\hline $\mathrm{C}(=\mathrm{C})(\mathrm{Cl}) \mathrm{Cl}$ & 2.13 & 2.12 & -0.01 & 1.97 & -0.16 \\
\hline c1ccc2ccnc2c1 & 2.14 & 2.05 & -0.09 & 2.29 & 0.1 \\
\hline $\mathrm{BrC}(\mathrm{C}) \mathrm{C}$ & 2.14 & 2.08 & -0.06 & 1.83 & -0.31 \\
\hline $\mathrm{Oc}(\mathrm{c}(\mathrm{ccc} 1) \mathrm{Cl}) \mathrm{c} 1$ & 2.15 & 2.16 & 0.01 & 2.40 & 0.25 \\
\hline $\mathrm{N}(\mathrm{c}(\operatorname{ccc}(\mathrm{c} 1) \mathrm{C}) \mathrm{c} 1) \mathrm{C}$ & 2.15 & 2.17 & 0.02 & 2.10 & -0.0 \\
\hline $\mathrm{FC}(\mathrm{F})(\mathrm{Cl}) \mathrm{Cl}$ & 2.16 & 1.82 & -0.34 & 2.06 & -0.10 \\
\hline $\begin{array}{l}\mathrm{CCCC} 1 \mathrm{CC}(\mathrm{N}(\mathrm{C}) \mathrm{C} 1) \mathrm{C}(=\mathrm{O}) \mathrm{NC}(\mathrm{C}(\mathrm{C}) \mathrm{Cl} \\
) \mathrm{C} 2 \mathrm{OC}(\mathrm{SC}) \mathrm{C}(\mathrm{O}) \mathrm{C}(\mathrm{O}) \mathrm{C} 2 \mathrm{O}\end{array}$ & 2.16 & 2.01 & -0.15 & 1.76 & \\
\hline $\mathrm{O}(\mathrm{Cc}(\operatorname{cccc} 1) \mathrm{c} 1) \mathrm{CC}$ & 2.16 & 2.27 & 0.11 & 2.38 & 0.22 \\
\hline $\mathrm{N}(=0)(=0) c(\operatorname{ccc}(\operatorname{c1N}(=0)(=0)) \mathrm{Cl}) \mathrm{c} 1$ & 2.17 & 2.27 & 0.10 & 2.29 & 0.12 \\
\hline $\begin{array}{l}\mathrm{COc} 1 \mathrm{c}(\mathrm{Cl}) \mathrm{c} 2 \mathrm{OC}(\mathrm{C}(\mathrm{OC})=\mathrm{C} 3) \\
(\mathrm{C}(\mathrm{C}) \mathrm{CC} 3=\mathrm{O}) \mathrm{C}(=\mathrm{O}) \mathrm{c} 2 \mathrm{c}(\mathrm{OC}) \mathrm{c} 1\end{array}$ & 2.18 & 1.92 & -0.26 & 2.71 & \\
\hline $\mathrm{O}=\mathrm{C}(\mathrm{C}=\mathrm{Cc}(\operatorname{ccc} 1) \mathrm{c} 1) \mathrm{C}$ & 2.18 & 2.04 & -0.14 & 2.23 & 0.05 \\
\hline $\mathrm{O}=\mathrm{C}(\mathrm{N}(\mathrm{CC}) \mathrm{CC}) \mathrm{c}(\operatorname{cccc} 1 \mathrm{C}) \mathrm{c} 1$ & 2.18 & 2.26 & 0.08 & 2.10 & -0.0 \\
\hline $\mathrm{BrCCCCl}$ & 2.18 & 2.41 & 0.23 & 2.12 & -0.06 \\
\hline $\mathrm{C} 1(=\mathrm{O}) \mathrm{C}(\mathrm{NC})(\mathrm{c} 2 \mathrm{c}(\mathrm{Cl}) \mathrm{ccc} 2) \mathrm{CCCC} 1$ & 2.18 & 3.12 & 0.94 & 2.69 & 0.5 \\
\hline $\begin{array}{l}\mathrm{CN} 2 \mathrm{C}(=\mathrm{O}) \mathrm{C}(\mathrm{O}) \mathrm{N}=\mathrm{C}(\mathrm{c} 1 \mathrm{ccccc} 1) \mathrm{c} 3 \mathrm{cc}( \\
\mathrm{Cl}) \operatorname{ccc} 23\end{array}$ & 2.19 & 2.15 & -0.04 & 2.16 & 0.0 \\
\hline $\mathrm{O}=\mathrm{C}(\mathrm{c}(\mathrm{cccc} 1) \mathrm{c} 1) \mathrm{CC}$ & 2.19 & 2.16 & -0.03 & 2.15 & \\
\hline $\begin{array}{l}\mathrm{C} 1 \mathrm{C}(=\mathrm{O}) \mathrm{C}=\mathrm{C} 2 \mathrm{CCC} 3 \mathrm{C} 4 \mathrm{CCC}(\mathrm{O}) \\
(\mathrm{C}(=\mathrm{O}) \mathrm{COC}(=\mathrm{O}) \mathrm{C}) \mathrm{C} 4(\mathrm{C}) \mathrm{CC}(\mathrm{O}) \mathrm{C} 3 \mathrm{C} 2 \\
(\mathrm{C}) \mathrm{C} 1\end{array}$ & 2.19 & 2.36 & 0.17 & 2.31 & \\
\hline$c 1 c c 2 C(=O) C=C(C) C(=0) c 2 c c 1$ & 2.20 & 2.21 & 0.01 & 1.91 & 0.2 \\
\hline
\end{tabular}


3,5-pyridinedicarboxylic acid, 1,4-dihydro-2,6dimethyl-4-(2-nitrophenyl)-, dimethyl ester benzene, 1,3-dimethoxy-

4-heptanol

propanenitrile, 2-[[4-chloro-6-(ethylamino)-1,3,5triazin-2-yl]amino]-2-methyl-

bicyclo[3.1.1]hept-3-en-2-one, 4,6,6-trimethyl-

2,3-naphthalenediol

3-heptanol

2H-1,4-benzodiazepin-2-one, 7-chloro-1,3-dihydro-

3-hydroxy-5-phenyl-

1-naphthaleneacetic acid

1,4-benzenedicarboxylic acid, dimethyl ester

1-naphthalenamine

2H-1,4-benzodiazepin-2-one, 1,3-dihydro-7-nitro-5phenyl-

benzenamine, 4-bromo-

benzoic acid, 2-hydroxy-

benzoic acid, anhydride

benzoic acid, 4-methoxy-, methyl ester

2-propenoic acid, 2-methyl-, 2-hydroxy-3-

phenoxypropyl ester

benzenesulfonamide, 4-chloro- $\mathrm{N}$ -

[(propylamino)carbonyl]-

3-pyridinecarboxylic acid, butyl ester

benzene, fluoro-

benzoic acid, 4-methyl-

propane, 1,2,3-trichloro-

phenol, 2-methoxy-4-(2-propenyl)-

2-naphthalenamine

1-butanethiol

2-naphthalenol, 1-nitroso-

benzeneacetic acid, ethyl ester

acetamide, $\mathrm{N}$-(4-bromophenyl)-

pyrazine, 2-methyl-6-propoxy-

3,5-pyrazolidinedione, 1,2-diphenyl-4-[2-

(phenylsulfinyl)ethyl]-

benzeneacetic acid, alpha-hydroxy-alpha-phenyl-

ethane, 2-bromo-2-chloro-1,1,1-trifluoro-

acetic acid, pentyl ester

methane, diiodo-

benzene, 1-methyl-2-nitro-

acetic acid, 2-phenylethyl ester

phenol, 2,4-dimethyl-

benzene, chloromethyl-

$1 \mathrm{H}$-indole-3-butanoic acid

$1 \mathrm{H}$-indole-2-carboxylic acid

benzenamine, $\mathrm{N}, \mathrm{N}$-dimethyl-

2-heptanol

ethanone, 1-(4-chlorophenyl)-

benzenepropanoic acid, methyl ester

2-butene

8-quinolinol, 2-methyl-

benzaldehyde, 2-chloro-

thiophene, 2-methyl-

butane, 2-chloro-

phenol, 2,5-dimethyl-

1H-2,1,3-benzothiadiazin-4(3H)-one, 3-(1-

methylethyl)-, 2,2-dioxide

1-propene, 2-methyl-

benzenamine, 4-iodo-

thiophene, 3-methyl-

benzenesulfonamide, $\mathrm{N}$-[(butylamino)carbonyl]-4methyl-

[1,1-biphenyl]-4,4-diamine, 3,3-dimethyl-

benzenebutanol

phenol, 3,5-dimethyl-

\begin{tabular}{|c|c|c|c|c|c|}
\hline $\begin{array}{l}\operatorname{COC}(=O) C 1=C(C) N C(=C(C 1 c 2 c c c c c \\
2 N(=O)(=O)) C(=O) O C) C\end{array}$ & 2.20 & 2.50 & 0.30 & 2.49 & 0.29 \\
\hline $\mathrm{O}(\mathrm{c}(\operatorname{cccc} 10 \mathrm{O}) \mathrm{c} 1) \mathrm{C}$ & 2.21 & 2.15 & -0.06 & 2.00 & -0.21 \\
\hline $\mathrm{OC}(\mathrm{CCC}) \mathrm{CCC}$ & 2.22 & 2.24 & 0.02 & 2.26 & 0.04 \\
\hline $\mathrm{CCNc} 1 \mathrm{nc}(\mathrm{Cl}) \mathrm{nc}(\mathrm{NC}(\mathrm{C})(\mathrm{C}) \mathrm{C}(\# \mathrm{~N})) \mathrm{n} 1$ & 2.22 & 2.51 & 0.29 & 2.05 & -0.17 \\
\hline $\mathrm{O}=\mathrm{C}(\mathrm{C}=\mathrm{C}(\mathrm{C}) \mathrm{C} 12) \mathrm{C}(\mathrm{C} 2) \mathrm{C} 1(\mathrm{C}) \mathrm{C}$ & 2.23 & 3.21 & 0.98 & 2.30 & 0.07 \\
\hline $\mathrm{Oc}(\mathrm{c}(\mathrm{O}) \mathrm{cc}(\mathrm{c} 1 \mathrm{ccc} 2) \mathrm{c} 2) \mathrm{c} 1$ & 2.24 & 2.21 & -0.03 & 2.08 & -0.16 \\
\hline $\mathrm{OC}(\mathrm{CCCC}) \mathrm{CC}$ & 2.24 & 2.24 & 0.00 & 2.29 & 0.05 \\
\hline $\begin{array}{l}\mathrm{OC} 3 \mathrm{~N}=\mathrm{C}(\mathrm{c} 1 \mathrm{ccccc} 1) \mathrm{c} 2 \mathrm{cc}(\mathrm{Cl}) \mathrm{ccc} 2 \mathrm{NC} 3 \\
(=\mathrm{O})\end{array}$ & 2.24 & 2.32 & 0.08 & 2.01 & -0.23 \\
\hline $\mathrm{O}=\mathrm{C}(\mathrm{O}) \mathrm{Cc}(\mathrm{c}(\mathrm{c}(\mathrm{ccc} 1) \mathrm{cc} 2) \mathrm{c} 1) \mathrm{c} 2$ & 2.24 & 2.60 & 0.36 & 2.97 & 0.73 \\
\hline $\mathrm{O}=\mathrm{C}(\mathrm{OC}) \mathrm{c}(\operatorname{ccc}(\mathrm{c} 1) \mathrm{C}(=\mathrm{O}) \mathrm{OC}) \mathrm{c} 1$ & 2.25 & 1.66 & -0.59 & 1.83 & -0.42 \\
\hline$c(c(c(N) c c 1) \operatorname{ccc} 2)(c 2) c 1$ & 2.25 & 2.25 & 0.00 & 2.27 & 0.02 \\
\hline $\begin{array}{l}\mathrm{O}=\mathrm{C} 3 \mathrm{CN}=\mathrm{C}(\mathrm{c} 1 \mathrm{ccc} c \mathrm{c} 1) \mathrm{c} 2 \mathrm{cc}(\operatorname{ccc} 2 \mathrm{~N} 3) \\
\mathrm{N}(=\mathrm{O})=\mathrm{O}\end{array}$ & 2.25 & 2.45 & 0.20 & 1.95 & -0.30 \\
\hline $\begin{array}{l}\mathrm{N}(=\mathrm{O})=\mathrm{O} \\
\mathrm{Nc}(\operatorname{ccc}(\mathrm{c} 1) \mathrm{Br}) \mathrm{c} 1\end{array}$ & & & & & \\
\hline $\mathrm{Nc}(\mathrm{ccc}(\mathrm{c} 1) \mathrm{Br}) \mathrm{c} 1$ & 2.26 & 1.97 & -0.29 & 2.10 & -0.16 \\
\hline $\mathrm{O}=\mathrm{C}(\mathrm{O}) \mathrm{c}(\mathrm{c}(\mathrm{O}) \mathrm{ccc} 1) \mathrm{c} 1$ & 2.26 & 2.24 & -0.02 & 1.96 & -0.30 \\
\hline $\mathrm{O}=\mathrm{C}(\mathrm{OC}(=\mathrm{O}) \mathrm{c}(\operatorname{cccc} 1) \mathrm{c} 1) \mathrm{c}(\operatorname{ccc} 2) \mathrm{c} 2$ & 2.26 & 2.33 & 0.07 & 3.22 & 0.96 \\
\hline $\mathrm{O}=\mathrm{C}(\mathrm{OC}) \mathrm{c}(\operatorname{ccc}(\mathrm{OC}) \mathrm{c} 1) \mathrm{c} 1$ & 2.27 & 1.91 & -0.36 & 2.05 & -0.22 \\
\hline $\mathrm{C}=\mathrm{C}(\mathrm{C}) \mathrm{C}(=\mathrm{O}) \mathrm{OCC}(\mathrm{O}) \mathrm{COc} 1 \mathrm{ccccc} 1$ & 2.27 & 1.96 & -0.31 & 1.83 & -0.44 \\
\hline $\begin{array}{l}\operatorname{c1cc}(\mathrm{Cl}) \operatorname{ccc} 1 \mathrm{~S}(=\mathrm{O}) \\
(=\mathrm{O}) \mathrm{NC}(=\mathrm{O}) \mathrm{NCCC}\end{array}$ & 2.27 & 2.01 & -0.26 & 2.15 & -0.12 \\
\hline $\mathrm{O}=\mathrm{C}(\mathrm{OCCCC}) \mathrm{c}(\operatorname{ccc} 1) \mathrm{c} 1$ & 2.27 & 2.11 & -0.16 & 2.23 & -0.04 \\
\hline $\mathrm{Fc}(\mathrm{cccc} 1) \mathrm{c} 1$ & 2.27 & 2.19 & -0.08 & 2.18 & -0.09 \\
\hline $\mathrm{O}=\mathrm{C}(\mathrm{O}) \mathrm{c}(\operatorname{ccc}(\mathrm{c} 1) \mathrm{C}) \mathrm{c} 1$ & 2.27 & 2.42 & 0.15 & 2.12 & -0.15 \\
\hline $\mathrm{ClCC}(\mathrm{Cl}) \mathrm{CCl}$ & 2.27 & 2.50 & 0.23 & 2.29 & 0.02 \\
\hline $\mathrm{O}(\mathrm{c}(\mathrm{c}(\mathrm{O}) \operatorname{ccc} 1 \mathrm{CC}=\mathrm{C}) \mathrm{c} 1) \mathrm{C}$ & 2.27 & 2.73 & 0.46 & 2.66 & 0.39 \\
\hline$c(c(\operatorname{ccc} 1 N) \operatorname{ccc} 2)(c 2) c 1$ & 2.28 & 2.25 & -0.03 & 2.30 & 0.02 \\
\hline SCCCC & 2.28 & 2.25 & -0.03 & 2.51 & 0.23 \\
\hline $\mathrm{O}=\mathrm{Nc}(\mathrm{c}(\mathrm{c}(\mathrm{ccc} 1) \mathrm{cc} 2) \mathrm{c} 1) \mathrm{c} 2 \mathrm{O}$ & 2.28 & 2.56 & 0.28 & 2.50 & 0.22 \\
\hline $\mathrm{O}=\mathrm{C}(\mathrm{OCC}) \mathrm{Cc}(\operatorname{cccc} 1) \mathrm{c} 1$ & 2.28 & 2.57 & 0.29 & 2.48 & 0.20 \\
\hline $\mathrm{O}=\mathrm{C}(\mathrm{Nc}(\mathrm{ccc}(\mathrm{c} 1) \mathrm{Br}) \mathrm{c} 1) \mathrm{C}$ & 2.29 & 1.99 & -0.30 & 1.88 & -0.41 \\
\hline $\mathrm{n} 1 \mathrm{c}(\mathrm{C}) \mathrm{cncc} 1 \mathrm{OCCC}$ & 2.29 & 2.45 & 0.16 & 2.08 & -0.21 \\
\hline $\begin{array}{l}\mathrm{O}=\mathrm{C} 2 \mathrm{C}(\mathrm{CCS}(=\mathrm{O}) \operatorname{c} 1 \operatorname{ccc} c 1) \mathrm{C}(=\mathrm{O}) \mathrm{N}( \\
\mathrm{N} 2 \mathrm{c} 3 \operatorname{ccc} c 3) \mathrm{c} 4 \operatorname{ccc} c 4\end{array}$ & 2.30 & 2.14 & -0.16 & 2.92 & 0.62 \\
\hline $\mathrm{O}=\mathrm{C}(\mathrm{O}) \mathrm{C}(\mathrm{O})(\mathrm{c}(\mathrm{cccc} 1) \mathrm{c} 1) \mathrm{c}(\mathrm{ccc} 2) \mathrm{c} 2$ & 2.30 & 2.24 & -0.06 & 2.12 & -0.18 \\
\hline $\mathrm{FC}(\mathrm{F})(\mathrm{F}) \mathrm{C}(\mathrm{Cl}) \mathrm{Br}$ & 2.30 & 2.26 & -0.04 & 2.50 & 0.20 \\
\hline $\mathrm{O}=\mathrm{C}(\mathrm{Occcc}) \mathrm{C}$ & 2.30 & 2.34 & 0.04 & 2.46 & 0.16 \\
\hline$C(I) I$ & 2.30 & 2.35 & 0.05 & 2.25 & -0.05 \\
\hline $\mathrm{N}(=\mathrm{O})(=\mathrm{O}) \mathrm{c}(\mathrm{c}(\operatorname{ccc} 1) \mathrm{C}) \mathrm{c} 1$ & 2.30 & 2.36 & 0.06 & 2.32 & 0.02 \\
\hline $\mathrm{O}=\mathrm{C}(\mathrm{OCCc}(\operatorname{cccc} 1) \mathrm{c} 1) \mathrm{C}$ & 2.30 & 2.57 & 0.27 & 2.38 & 0.08 \\
\hline $\mathrm{Oc}(\mathrm{c}(\mathrm{cc}(\mathrm{c} 1) \mathrm{C}) \mathrm{C}) \mathrm{c} 1$ & 2.30 & 2.61 & 0.31 & 2.37 & 0.07 \\
\hline CICc1 $1 \operatorname{ccccc} 1$ & 2.30 & 2.79 & 0.49 & 2.51 & 0.21 \\
\hline $\mathrm{O}=\mathrm{C}(\mathrm{O}) \mathrm{CCCC}(\mathrm{c}(\mathrm{c}(\mathrm{N} 1) \mathrm{ccc} 2) \mathrm{c} 2)=\mathrm{C} 1$ & 2.30 & 2.84 & 0.54 & 2.38 & 0.08 \\
\hline $\operatorname{c1ccc2cc}(\mathrm{C}(=0) \mathrm{O}) \mathrm{nc} 2 \mathrm{c} 1$ & 2.31 & 1.94 & -0.37 & 1.79 & -0.52 \\
\hline $\mathrm{N}(\mathrm{c}(\mathrm{cccc} 1) \mathrm{c} 1)(\mathrm{C}) \mathrm{C}$ & 2.31 & 2.17 & -0.14 & 2.05 & -0.26 \\
\hline $\mathrm{OC}(\mathrm{CCCCC}) \mathrm{C}$ & 2.31 & 2.24 & -0.07 & 2.34 & 0.03 \\
\hline $\mathrm{O}=\mathrm{C}(\mathrm{c}(\mathrm{ccc}(\mathrm{c} 1) \mathrm{Cl}) \mathrm{c} 1) \mathrm{C}$ & 2.32 & 2.32 & 0.00 & 2.41 & 0.09 \\
\hline $\mathrm{O}=\mathrm{C}(\mathrm{OC}) \mathrm{CCc}(\operatorname{cccc} 1) \mathrm{c} 1$ & 2.32 & 2.57 & 0.25 & 2.35 & 0.03 \\
\hline$C(=C C) C$ & 2.33 & 2.09 & -0.24 & 2.32 & -0.01 \\
\hline $\mathrm{n}(\mathrm{c}(\mathrm{c}(\mathrm{ccc} 1) \mathrm{cc} 2) \mathrm{c} 1 \mathrm{O}) \mathrm{c} 2 \mathrm{C}$ & 2.33 & 2.21 & -0.12 & 2.52 & 0.19 \\
\hline $\mathrm{O}=\mathrm{Cc}(\mathrm{c}(\mathrm{ccc} 1) \mathrm{Cl}) \mathrm{c} 1$ & 2.33 & 2.35 & 0.02 & 2.23 & -0.10 \\
\hline $\mathrm{C} 1=\mathrm{C}(\mathrm{C}) \mathrm{SC}=\mathrm{C} 1$ & 2.33 & 2.36 & 0.03 & 2.30 & -0.03 \\
\hline $\mathrm{C}(\mathrm{Cl})(\mathrm{CC}) \mathrm{C}$ & 2.33 & 2.49 & 0.16 & 2.34 & 0.01 \\
\hline $\mathrm{Oc}(\mathrm{c}(\operatorname{ccc} 1 \mathrm{C}) \mathrm{C}) \mathrm{c} 1$ & 2.33 & 2.61 & 0.28 & 2.35 & 0.02 \\
\hline $\begin{array}{l}\mathrm{O}=\mathrm{C}(\mathrm{N}(\mathrm{S}(=\mathrm{O}) \\
(=\mathrm{O}) \mathrm{Nc} 1 \mathrm{cccc} 2) \mathrm{C}(\mathrm{C}) \mathrm{C}) \mathrm{c} 12\end{array}$ & 2.34 & 1.67 & -0.67 & 1.86 & -0.48 \\
\hline $\mathrm{C}(=\mathrm{C})(\mathrm{C}) \mathrm{C}$ & 2.34 & 2.23 & -0.11 & 1.87 & -0.47 \\
\hline $\mathrm{Nc}(\mathrm{ccc}(\mathrm{c} 1) \mathrm{I}) \mathrm{c} 1$ & 2.34 & 2.24 & -0.10 & 2.58 & 0.24 \\
\hline $\mathrm{C} 1(\mathrm{C})=\mathrm{CSC}=\mathrm{C} 1$ & 2.34 & 2.36 & 0.02 & 2.28 & -0.06 \\
\hline $\begin{array}{l}\mathrm{O}=\mathrm{C}(\mathrm{NCCCC}) \mathrm{NS}(=\mathrm{O}) \\
(=\mathrm{O}) \mathrm{c}(\operatorname{ccc}(\mathrm{c} 1) \mathrm{C}) \mathrm{c} 1\end{array}$ & 2.34 & 2.41 & 0.07 & 2.04 & -0.30 \\
\hline $\mathrm{Nc} 1 \mathrm{c}(\mathrm{C}) \mathrm{cc}(\mathrm{c} 2 \mathrm{cc}(\mathrm{C}) \mathrm{c}(\mathrm{N}) \mathrm{cc} 2) \mathrm{cc} 1$ & 2.34 & 3.02 & 0.68 & 2.44 & 0.10 \\
\hline OCCCCc(cccc1)c1 & 2.35 & 2.55 & 0.20 & 2.45 & 0.10 \\
\hline $\mathrm{Oc}(\operatorname{cc}(\operatorname{cc} 1 \mathrm{C}) \mathrm{C}) \mathrm{c} 1$ & 2.35 & 2.61 & 0.26 & 2.38 & 0.03 \\
\hline
\end{tabular}


propane

2-propenoic acid, butyl ester

butanedioic acid, [(dimethoxyphosphinothioyl)thio]-,

diethylester

phenol, 2,6-dimethyl-

2-octanone

benzene, 1-methyl-4-nitro-

benzoic acid, 3-methyl-

propane, 1,3-dibromo-

androst-4-en-3-one, 9-fluoro-11,17-dihydroxy-17-

methyl-, (11-beta,17-beta)-

bicyclo[2.2.1] heptan-2-one, 1,7,7-trimethyl-

phenol, 4-chloro-

ethane, 1,1,2,2-tetrachloro-

benzoic acid, 2-mercapto-

2H-1,4-benzodiazepin-2-one, 7-chloro-5-(2-

chlorophenyl)-1,3-dihydro-3-hydroxy-

benzene, 1-chloro-4-nitro-

methane, tribromo-

pregna-1,4-diene-3,20-dione, 21-(acetyloxy)-11,17

dihydroxy-, (11)-

butene

furan, 2-ethyl-

phenol, 3-ethyl-

benzoic acid, 2-iodo-

2H-1,4-benzodiazepin-2-one, 5-(2-chlorophenyl)-

1,3-dihydro-7-nitro-

urea, N'-(3-chloro-4-methylphenyl)-N,N-dimethyl-

ethene, trichloro-

heptanoic acid

1,3-butadiene, 2-methyl-

1,2-benzenedicarboxylic acid, diethyl ester

benzenebutanoic acid

2(3H)-benzothiazolethione

$4 \mathrm{H}-[1,2,4]$ triazolo[4,3-a][1,4]benzodiazepine, 8-

chloro-6-(2-chlorophenyl)-1-methyl-

2-furanmethanol, 5-ethenyltetrahydro-alpha,alpha-

5-trimethyl-

acetamide, 2-(diethylamino)-N-(2,6-

dimethylphenyl)-

$3 \mathrm{H}-1,4-$ benzodiazepin-2-amine, 7-chloro-N-methyl-

5-phenyl-, 4-oxide

1,3-pentadiene

benzenemethanol, 4-methyl-, acetate

$5 \mathrm{H}$-dibenz[b,f]azepine-5-carboxamide

benzene, 1-methyl-3-nitro-

$3(2 \mathrm{H})$-isothiazolone, 2-octyl-

estra-1,3,5(10)-triene-3,16,17-triol, (16-alpha,17 )-

benzoic acid, 2-methyl-

phenol, 4-chloro-2-nitro-

2,4-imidazolidinedione, 5,5-diphenyl-

benzoic acid, 4-hydroxy-, ethyl ester

phenol, 2-ethyl-

1,4-pentadiene

pregna-1,4-diene-3,20-dione, 6,9-difluoro-11,21-

dihydroxy-16,17-[(1-methylethylidene)bis(oxy)]-, (6alpha,1-beta,1-alpha))-

phenol, 2,3-dimethyl-

4-thia-1-azabicyclo[3.2.0]heptane-2-carboxylic acid,

6-[[[3-(2-chlorophenyl)-5-methyl-4-

isoxazolyl]carbonyl]amino]-3,3-dimethyl-7-oxo-

ethane, 1,1,1-trichloro-

phenol, 3-chloro-

benzenepropanoic acid, alpha-acetyl-, ethyl ester
$\mathrm{C}(\mathrm{C}) \mathrm{C}$

$\mathrm{O}=\mathrm{C}(\mathrm{OCCCC}) \mathrm{C}=\mathrm{C}$

$\operatorname{CCOC}(=0) \operatorname{CC}(\operatorname{SP}(=\mathrm{S})$

$(\mathrm{OC}) \mathrm{OC}) \mathrm{C}(=\mathrm{O}) \mathrm{OCC}$

$\mathrm{Oc}(\mathrm{c}(\mathrm{ccc} 1) \mathrm{C}) \mathrm{c} 1 \mathrm{C}$

$\mathrm{O}=\mathrm{C}(\mathrm{CCCCCC}) \mathrm{C}$

$\mathrm{N}(=\mathrm{O})(=\mathrm{O}) \mathrm{c}(\operatorname{ccc}(\mathrm{c} 1) \mathrm{C}) \mathrm{c} 1$

$\mathrm{O}=\mathrm{C}(\mathrm{O}) \mathrm{c}(\operatorname{ccc} 1 \mathrm{C}) \mathrm{c} 1$

$\mathrm{BrCCCBr}$

$\mathrm{CC} 1(\mathrm{O}) \mathrm{CCC} 2 \mathrm{C} 3 \mathrm{CCC} 4=\mathrm{CC}(=\mathrm{O}) \mathrm{CCC}$

4(C)C3(F)C $(\mathrm{O}) \mathrm{CC} 21 \mathrm{C}$

$\mathrm{O}=\mathrm{C}(\mathrm{C}(\mathrm{C}(\mathrm{C} 1 \mathrm{C} 2)(\mathrm{C}) \mathrm{C})(\mathrm{C} 2) \mathrm{C}) \mathrm{C} 1$

$\mathrm{Oc}(\mathrm{ccc}(\mathrm{c} 1) \mathrm{Cl}) \mathrm{c} 1$

$\mathrm{C}(\mathrm{C}(\mathrm{Cl}) \mathrm{Cl})(\mathrm{Cl}) \mathrm{Cl}$

$\mathrm{O}=\mathrm{C}(\mathrm{O}) \mathrm{c}(\mathrm{c}(\mathrm{S}) \mathrm{ccc} 1) \mathrm{c} 1$

OC $3 \mathrm{~N}=\mathrm{C}(\mathrm{c} 1 \mathrm{ccccc} 1 \mathrm{Cl}) \mathrm{c} 2 \mathrm{cc}(\mathrm{Cl}) \mathrm{ccc} 2 \mathrm{~N}$

$\mathrm{C} 3=\mathrm{O}$

$\mathrm{N}(=\mathrm{O})(=\mathrm{O}) \mathrm{c}(\mathrm{ccc}(\mathrm{c} 1) \mathrm{Cl}) \mathrm{c} 1$

$\mathrm{BrC}(\mathrm{Br}) \mathrm{Br}$

$\mathrm{O}=\mathrm{C} 1 \mathrm{C}=\mathrm{C} 2 \mathrm{CCC} 3 \mathrm{C} 4 \mathrm{CCC}(\mathrm{O})$

$(\mathrm{C}(=\mathrm{O}) \mathrm{COC}(=\mathrm{O}) \mathrm{C}) \mathrm{C} 4(\mathrm{C}) \mathrm{CC}(\mathrm{O}) \mathrm{C} 3 \mathrm{C} 2$

$(\mathrm{C}) \mathrm{C}=\mathrm{C} 1$

$\mathrm{C}=\mathrm{CCC}$

$\mathrm{C} 1=\mathrm{COC}(\mathrm{CC})=\mathrm{C} 1$

$\mathrm{Oc} 1 \mathrm{cc}(\mathrm{CC}) \operatorname{ccc} 1$

$\mathrm{O}=\mathrm{C}(\mathrm{O}) \mathrm{c}(\mathrm{c}(\mathrm{ccc} 1) \mathrm{I}) \mathrm{c} 1$

$\mathrm{Clc} 1 \mathrm{ccccc} 1 \mathrm{C} 2=\mathrm{NCC}(=\mathrm{O}) \mathrm{Nc} 3 \mathrm{ccc}(\mathrm{cc} 2$

3) $\mathrm{N}(=\mathrm{O})=\mathrm{O}$

$\mathrm{CN}(\mathrm{C}) \mathrm{C}(=\mathrm{O}) \mathrm{Nc} 1 \mathrm{ccc}(\mathrm{C}) \mathrm{c}(\mathrm{Cl}) \mathrm{c} 1$

$\mathrm{C}(=\mathrm{CCl})(\mathrm{Cl}) \mathrm{Cl}$

$\mathrm{O}=\mathrm{C}(\mathrm{O}) \mathrm{CCCCCC}$

$\mathrm{C}(\mathrm{C}=\mathrm{C})(=\mathrm{C}) \mathrm{C}$

$\mathrm{O}=\mathrm{C}(\mathrm{OCC}) \mathrm{c}(\mathrm{c}(\mathrm{ccc} 1) \mathrm{C}(=\mathrm{O}) \mathrm{OCC}) \mathrm{c} 1$

$\mathrm{O}=\mathrm{C}(\mathrm{O}) \mathrm{CCCc}(\operatorname{cccc} 1) \mathrm{c} 1$

$\mathrm{N}(\mathrm{c}(\mathrm{c}(\mathrm{S} 1) \mathrm{ccc} 2) \mathrm{c} 2)=\mathrm{C} 1 \mathrm{~S}$

$\mathrm{c} 1 \mathrm{cc} 2 \mathrm{n} 3 \mathrm{c}(\mathrm{C}) \mathrm{nnc} 3 \mathrm{CN}=\mathrm{C}(\mathrm{c} 4 \mathrm{c}(\mathrm{Cl}) \mathrm{cccc} 4$ $\mathrm{c} 2 \mathrm{cc} 1 \mathrm{Cl}$

$\mathrm{O}(\mathrm{C}(\mathrm{C}=\mathrm{C})(\mathrm{CC} 1) \mathrm{C}) \mathrm{C} 1 \mathrm{C}(\mathrm{O})(\mathrm{C}) \mathrm{C}$

$\mathrm{O}=\mathrm{C}(\mathrm{Nc}(\mathrm{c}(\mathrm{ccc} 1) \mathrm{C}) \mathrm{c} 1 \mathrm{C}) \mathrm{CN}(\mathrm{CC}) \mathrm{CC}$

$\mathrm{CNC2}=\mathrm{Nc} 1 \mathrm{ccc}(\mathrm{Cl}) \operatorname{cc} 1 \mathrm{C}(=\mathrm{N}(=\mathrm{O}) \mathrm{C} 2) \mathrm{c}$

3cccce 3

$\mathrm{C}(=\mathrm{CC}=\mathrm{C}) \mathrm{C}$

$\mathrm{O}=\mathrm{C}(\mathrm{OCc}(\operatorname{ccc}(\mathrm{c} 1) \mathrm{C}) \mathrm{c} 1) \mathrm{C}$

$\mathrm{NC}(=\mathrm{O}) \mathrm{N} 2 \mathrm{c} 1 \mathrm{ccccc} 1 \mathrm{C}=\mathrm{Cc} 3 \operatorname{ccccc} 23$

$\mathrm{N}(=\mathrm{O})(=\mathrm{O}) \mathrm{c}(\operatorname{cccc} 1 \mathrm{C}) \mathrm{c} 1$

$\mathrm{O}=\mathrm{C} 1 \mathrm{C}=\mathrm{CSN} 1 \mathrm{CCCCCCCC}$

CC34CCC1C $(\mathrm{CCc} 2 \mathrm{cc}(\mathrm{O}) \operatorname{ccc} 12) \mathrm{C} 3 \mathrm{C}$

$\mathrm{C}(\mathrm{O}) \mathrm{C} 4 \mathrm{O}$

$\mathrm{O}=\mathrm{C}(\mathrm{O}) \mathrm{c}(\mathrm{c}(\mathrm{ccc} 1) \mathrm{C}) \mathrm{c} 1$

$\mathrm{N}(=\mathrm{O})(=\mathrm{O}) \mathrm{c}(\mathrm{c}(\mathrm{O}) \mathrm{ccc} 1 \mathrm{Cl}) \mathrm{c} 1$

$\mathrm{O}=\mathrm{C} 1 \mathrm{NC}(=\mathrm{O}) \mathrm{C}(\mathrm{N} 1)$

(c2ccccc2)c3ccccc3

$\mathrm{O}=\mathrm{C}(\mathrm{OCC}) \mathrm{c}(\mathrm{ccc}(\mathrm{O}) \mathrm{c} 1) \mathrm{c} 1$

$\mathrm{Oc}(\mathrm{c}(\mathrm{ccc} 1) \mathrm{CC}) \mathrm{c} 1$

$\mathrm{C}(=\mathrm{C}) \mathrm{CC}=\mathrm{C}$

$\mathrm{C} 1(=\mathrm{O}) \mathrm{C}=\mathrm{C} 2 \mathrm{C}(\mathrm{F}) \mathrm{CC} 3 \mathrm{C} 4 \mathrm{CC} 5 \mathrm{C}(\mathrm{C}(=\mathrm{O}$

) $\mathrm{CO})(\mathrm{OC}(\mathrm{C})$

(C)O5) $\mathrm{C} 4(\mathrm{C}) \mathrm{CC}(\mathrm{O}) \mathrm{C} 3(\mathrm{~F}) \mathrm{C} 2(\mathrm{C}) \mathrm{C}=\mathrm{C} 1$

$\mathrm{Oc}(\mathrm{c}(\mathrm{c}(\mathrm{cc} 1) \mathrm{C}) \mathrm{C}) \mathrm{c} 1$

$\mathrm{N} 12 \mathrm{C}(=\mathrm{O}) \mathrm{C}(\mathrm{NC}(=\mathrm{O}) \mathrm{C} 4=\mathrm{C}(\mathrm{C}) \mathrm{ON}=\mathrm{C} 4$

c3c $(\mathrm{Cl}) \operatorname{cccc} 3) \mathrm{C} 1 \mathrm{SC}(\mathrm{C})(\mathrm{C}) \mathrm{C} 2 \mathrm{C}(=\mathrm{O}) \mathrm{O}$

$\mathrm{C}(\mathrm{Cl})(\mathrm{Cl})(\mathrm{Cl}) \mathrm{C}$

$\mathrm{Oc}(\mathrm{cccc} 1 \mathrm{Cl}) \mathrm{c} 1$

$\operatorname{CCOC}(=0) \mathrm{C}(\mathrm{Cc} 1 \mathrm{ccccc} 1) \mathrm{C}(=\mathrm{O}) \mathrm{C}$

2.39

2.40

2.44

2.44

2.46

2.47

2.47

2.50
2.36

1.81

2.20

$\begin{array}{lllll}2.36 & 2.29 & -0.07 & 2.67 & 0.31\end{array}$

$-0.55$

$-0.16$

$\begin{array}{lllll}2.36 & 2.29 & -0.07 & 2.67 & 0.31\end{array}$

2.19

2.20

$\begin{array}{lllll}2.36 & 2.29 & -0.07 & 2.67 & 0.31\end{array}$

$-0.17$

$\begin{array}{lllll}2.36 & 2.61 & 0.25 & 2.32 & -0.04\end{array}$

$\begin{array}{lllll}2.37 & 2.22 & -0.15 & 2.54 & 0.17\end{array}$

$\begin{array}{lllll}2.37 & 2.36 & -0.01 & 2.34 & -0.03\end{array}$

$\begin{array}{lllll}2.37 & 2.42 & 0.05 & 2.08 & -0.29\end{array}$

$\begin{array}{lllll}2.37 & 2.50 & 0.13 & 2.55 & 0.18\end{array}$

$\begin{array}{lllll}2.38 & 2.49 & 0.11 & 2.50 & 0.12\end{array}$

$\begin{array}{lllll}2.38 & 3.04 & 0.66 & 2.85 & 0.47\end{array}$

$\begin{array}{lllll}2.39 & 2.16 & -0.23 & 2.37 & -0.02\end{array}$

$\begin{array}{lllll}2.39 & 2.19 & -0.20 & 2.57 & 0.18\end{array}$

$\begin{array}{lllll}2.39 & 2.22 & -0.17 & 1.65 & -0.74\end{array}$

$\begin{array}{lllll}2.39 & 2.41 & 0.02 & 2.98 & 0.59\end{array}$

2.46

0.07

2.56

1.79

$-0.61$

$-0.26$

0.17

0.10

$-0.23$

2.17

$-0.23$

0.00

0.15

0.30

0.12

0.17

2.58

$2.42 \quad 2.47$

0.05

0.12

0.16

0.23

0.36

0.44

1.54

$-0.44$

2.43

1.99

2.44

1.66

$-0.78$

2.42

$-0.02$

2.45

2.63

2.25

$2.45 \quad 2.36$

$2.45 \quad 2.61$

$2.45 \quad 2.81$

2.08

2.55

$2.47 \quad 2.16$

2.49

2.55

2.52

2.56

0.19

$-0.20$

$-0.09$

0.16

0.36

$-0.38$

0.09

$-0.31$

2.56

2.26

0.02

2.76

2.45

$\begin{array}{ll}0.04 & 2.39\end{array}$

$0.08 \quad 2.47$

2.48

2.61

0.13

0.74

2.34

2.61

$-0.14$

0.13

2.49

2.68

0.19

2.45

$-0.04$

2.52

1.92

$-0.34$

2.35

$-0.15$

$\begin{array}{lll}-0.60 & 2.15 & -0.37\end{array}$ 
benzenethiol

butane, 2-chloro-2-methyl-

methane, trichlorofluoro-

1-butene, 4-bromo-

2-propenoic acid, 2-methyl-, phenylmethyl ester

thiophene, 2-chloro-

benzoic acid, 2-hydroxy-, methyl ester

phenol, 4-ethyl-, acetate

1-heptanamine

quinoline, 6-methyl-

benzoic acid, 2-amino-, ethyl ester

phenol, 4-ethyl-

quinoline, 2-methyl-

2-propanol, 1-chloro-, phosphate (3:1)

2H-1-benzopyran-2-one, 4-hydroxy-3-(3-oxo-1phenylbutyl)-

$1 \mathrm{H}$-indole, 3-methyl-

quinoline, 8-methyl-

quinoline, 4-methyl-

1,3,5-triazine-2,4-diamine, 6-chloro-N-ethyl-N'-(1methylethyl)-

1-heptanol

2-propenoic acid, 3-phenyl-, methyl ester

benzamide, N-phenyl-

benzoic acid, ethyl ester

butane, 1-chloro-

hexanoic acid, 2-ethyl-

1H-imidazole, 4,5-dihydro-2-(phenylmethyl)phenol, 2-iodo-

benzene, 1-methoxy-4-methyl-

2-propenoic acid, 2-methyl-, 2-methylpropyl ester benzofuran

2-butene, 2-methyl-

benzenemethanol, alpha-phenyl-

phenol, 2,3,6-trimethyl-

benzenemethanamine, $\mathrm{N}$-(phenylmethyl)-

2-propenoic acid, 3-(4-methoxyphenyl)-

benzoic acid, 3-chloro-

urea, N'-(3,4-dichlorophenyl)-N,N-dimethyl-

cinchonan-9-ol

benzenamine, 3,4-dichloro-

2,4-pyrimidinediamine, 5-(4-chlorophenyl)-6-ethylacetamide, $\mathrm{N}$-(4-bromo-3-chloro-2-methylphenyl)-

bicyclo[2.2.1] heptan-2-ol, 1,7,7-trimethyl-

benzoic acid, 4-methyl-, methyl ester

2-naphthalenol

1,5-benzothiazepin-4(5H)-one, 3-(acetyloxy)-5-[2(dimethylamino)ethyl]-2,3-dihydro-2-(4-

methoxyphenyl)-

2-cyclohexen-1-one, 2-methyl-5-(1-methylethenyl)2(3H)-furanone, 5-hexyldihydro-

benzenamine, 4-chloro-2-nitro-

4-piperidinecarboxylic acid, 1-methyl-4-phenyl-, ethyl ester

3,5-pyrazolidinedione, 4-butyl-1-(4-hydroxyphenyl)2-phenyl-

yohimban-16-alpha-carboxylic acid, 17-alpha-

hydroxy-, methyl ester

benzene, methyl-

benzenepropanoic acid, ethyl ester

phenol, 2,4,6-trimethyl-

benzene, 1-methoxy-2-methyl-

2-oxabicyclo[2.2.2]octane, 1,3,3-trimethyl-

thiourea, N,N-dibutyl-

butane, 1-bromo-

$\mathrm{Sc}(\mathrm{cccc} 1) \mathrm{c} 1$
$\mathrm{C}(\mathrm{CC})(\mathrm{Cl})(\mathrm{C}) \mathrm{C}$
$\mathrm{FC}(\mathrm{Cl})(\mathrm{Cl}) \mathrm{Cl}$
$\mathrm{BrCC}=\mathrm{C}$
$\mathrm{O}=\mathrm{C}(\mathrm{OCc}(\mathrm{cccc} 1) \mathrm{c} 1) \mathrm{C}(=\mathrm{C}) \mathrm{C}$
$\mathrm{C} 1=\mathrm{CC}=\mathrm{C}(\mathrm{Cl}) \mathrm{S1}$
$\mathrm{O}=\mathrm{C}(\mathrm{OC}) \mathrm{c}(\mathrm{c}(\mathrm{O}) \mathrm{ccc} 1) \mathrm{c} 1$
$\mathrm{CC}(=\mathrm{O}) \mathrm{Oc} 1 \mathrm{ccc}(\mathrm{CC}) \mathrm{cc} 1$
$\mathrm{NCCCCCC}$
$\mathrm{n}(\mathrm{c}(\mathrm{c}(\mathrm{cc}(\mathrm{c} 1) \mathrm{C}) \mathrm{cc} 2) \mathrm{c} 1) \mathrm{c} 2$
$\mathrm{O}=\mathrm{C}(\mathrm{OCC}) \mathrm{c}(\mathrm{c}(\mathrm{N}) \mathrm{ccc} 1) \mathrm{c} 1$
$\mathrm{Oc}(\mathrm{ccc}(\mathrm{c} 1) \mathrm{CC}) \mathrm{c} 1$
$\mathrm{n}(\mathrm{c}(\mathrm{c}(\mathrm{ccc} 1) \mathrm{cc} 2) \mathrm{c} 1) \mathrm{c} 2 \mathrm{C}$
$\mathrm{O}=\mathrm{P}(\mathrm{OC}(\mathrm{CCl}) \mathrm{C})$
$(\mathrm{OC}(\mathrm{CCl}) \mathrm{C}) \mathrm{OC}(\mathrm{CCl}) \mathrm{C}$
$\mathrm{c} 1 \mathrm{ccc} 2 \mathrm{C}(\mathrm{O})=\mathrm{C}(\mathrm{C}(\mathrm{c} 3 \mathrm{ccccc} 3) \mathrm{CC}(=\mathrm{O}) \mathrm{C}$
$\mathrm{H}(=\mathrm{O}) \mathrm{Oc} 2 \mathrm{c} 1$
$\mathrm{c} 1 \mathrm{ccc} 2 \mathrm{c}(\mathrm{C}) \mathrm{cnc} 2 \mathrm{c} 1$

$\mathrm{c} 1 \mathrm{ccc} 2 \mathrm{c}(\mathrm{C}) \mathrm{cnc} 2 \mathrm{c} 1$

$\mathrm{n}(\mathrm{c}(\mathrm{c}(\mathrm{ccc} 1) \mathrm{cc} 2) \mathrm{c} 1 \mathrm{C}) \mathrm{c} 2$

$\mathrm{n}(\mathrm{c}(\mathrm{c}(\mathrm{c}(\mathrm{c} 1) \mathrm{C}) \mathrm{ccc} 2) \mathrm{c} 2) \mathrm{c} 1$

$\mathrm{n}(\mathrm{c}(\mathrm{nc}(\mathrm{n} 1) \mathrm{NC}(\mathrm{C}) \mathrm{C}) \mathrm{NCC}) \mathrm{c} 1 \mathrm{Cl}$

OCCCCCCC

$\mathrm{O}=\mathrm{C}(\mathrm{OC}) \mathrm{C}=\mathrm{Cc}(\operatorname{cccc} 1) \mathrm{c} 1$

$\mathrm{O}=\mathrm{C}(\mathrm{Nc}(\mathrm{cccc} 1) \mathrm{c} 1) \mathrm{c}(\mathrm{cccc} 2) \mathrm{c} 2$

$\mathrm{O}=\mathrm{C}(\mathrm{OCC}) \mathrm{c}(\operatorname{cccc} 1) \mathrm{c} 1$

$\mathrm{CICCCC}$

$\mathrm{O}=\mathrm{C}(\mathrm{O}) \mathrm{C}(\mathrm{CCCC}) \mathrm{CC}$

c1 ccccc1CC2=NCCN2

Oc(c(ccc1)l)c1

$\mathrm{O}(\mathrm{c}(\mathrm{ccc}(\mathrm{c} 1) \mathrm{C}) \mathrm{c} 1) \mathrm{C}$

$\mathrm{O}=\mathrm{C}(\mathrm{OCC}(\mathrm{C}) \mathrm{C}) \mathrm{C}(=\mathrm{C}) \mathrm{C}$

$\mathrm{c} 1 \mathrm{ccc} 2 \mathrm{ccoc} 2 \mathrm{c} 1$

$\mathrm{C}(=\mathrm{CC})(\mathrm{C}) \mathrm{C}$

$\mathrm{OC}(\mathrm{c}(\operatorname{cccc} 1) \mathrm{c} 1) \mathrm{c}(\operatorname{cccc} 2) \mathrm{c} 2$

$\mathrm{Oc}(\mathrm{c}(\operatorname{ccc} 1 \mathrm{C}) \mathrm{C}) \mathrm{c} 1 \mathrm{C}$

$\mathrm{N}(\mathrm{Cc}(\operatorname{cccc} 1) \mathrm{c} 1) \mathrm{Cc}(\operatorname{cccc} 2) \mathrm{c} 2$

$\mathrm{O}=\mathrm{C}(\mathrm{O}) \mathrm{C}=\mathrm{Cc}(\operatorname{ccc}(\mathrm{OC}) \mathrm{c} 1) \mathrm{c} 1$

$\mathrm{O}=\mathrm{C}(\mathrm{O}) \mathrm{c}(\operatorname{cccc} 1 \mathrm{Cl}) \mathrm{c} 1$

$\mathrm{O}=\mathrm{C}(\mathrm{N}(\mathrm{C}) \mathrm{C}) \mathrm{Nc}(\operatorname{ccc}(\mathrm{c} 1 \mathrm{Cl}) \mathrm{Cl}) \mathrm{c} 1$

$\mathrm{OC}(\mathrm{c}(\mathrm{c}(\mathrm{c}(\mathrm{nc} 1) \mathrm{ccc} 2) \mathrm{c} 2) \mathrm{c} 1) \mathrm{C}(\mathrm{N}(\mathrm{CCC} 3$

$\mathrm{C} 4 \mathrm{C}=\mathrm{C}) \mathrm{C} 4) \mathrm{C} 3$

$\mathrm{Nc}(\operatorname{ccc}(\mathrm{c} 1 \mathrm{Cl}) \mathrm{Cl}) \mathrm{c} 1$

CCc1nc(N)nc(N)c1c2ccc(Cl)cc2

$\mathrm{CC}(=\mathrm{O}) \mathrm{Nc} 1 \mathrm{c}(\mathrm{C}) \mathrm{c}(\mathrm{Cl}) \mathrm{c}(\mathrm{Br}) \mathrm{cc} 1$

$\mathrm{OC}(\mathrm{C}(\mathrm{C}(\mathrm{C} 1 \mathrm{C} 2)(\mathrm{C}) \mathrm{C})(\mathrm{C} 2) \mathrm{C}) \mathrm{C} 1$

$\mathrm{O}=\mathrm{C}(\mathrm{OC}) \mathrm{c}(\operatorname{ccc}(\mathrm{c} 1) \mathrm{C}) \mathrm{c} 1$

$\mathrm{Oc}(\operatorname{ccc}(\mathrm{c} 1 \mathrm{ccc} 2) \mathrm{c} 2) \mathrm{c} 1$

$\operatorname{c} 1 \operatorname{ccc} 2 \mathrm{~N}(\mathrm{CCN}(\mathrm{C}) \mathrm{C}) \mathrm{C}(=\mathrm{O}) \mathrm{C}(\mathrm{OC}(=\mathrm{O})$

C) $\mathrm{C}(\mathrm{c} 3 \mathrm{ccc}(\mathrm{OC}) \mathrm{cc} 3) \mathrm{Sc} 2 \mathrm{c} 1$

$\mathrm{O}=\mathrm{C}(\mathrm{C}(=\operatorname{CcC} 1 \mathrm{C}(=\mathrm{C}) \mathrm{C}) \mathrm{C}) \mathrm{C} 1$

$\mathrm{O}=\mathrm{C}(\mathrm{OC}(\mathrm{C} 1) \mathrm{CCCCCC}) \mathrm{C} 1$

$\mathrm{N}(=\mathrm{O})(=\mathrm{O}) \mathrm{c}(\mathrm{c}(\mathrm{N}) \operatorname{ccc} 1 \mathrm{Cl}) \mathrm{c} 1$

$\mathrm{C} 1 \mathrm{CN}(\mathrm{C}) \operatorname{CCC} 1$ (c2)cccc2)C $(=\mathrm{O}) \mathrm{OCC}$

2.71

2.72

2.72

2.72

$\operatorname{Ccccc} 1 \mathrm{C}(=\mathrm{O}) \mathrm{N}(\mathrm{N}(\mathrm{C} 1=\mathrm{O}) \mathrm{c} 2 \operatorname{ccc}(\mathrm{O}) \mathrm{c}$ c2) $\mathrm{c} 3 \mathrm{ccccc} 3$

$\mathrm{COC}(=\mathrm{O}) \mathrm{C} 4 \mathrm{C}(\mathrm{O}) \mathrm{CCC} 5 \mathrm{CN} 3 \mathrm{CCc} 1 \mathrm{c}(\mathrm{n}$

c2ccccc12)C3CC45

$\mathrm{c}(\mathrm{cccc} 1)(\mathrm{c} 1) \mathrm{C}$

$\mathrm{O}=\mathrm{C}(\mathrm{OCC}) \mathrm{CC}(\operatorname{cccc} 1) \mathrm{c} 1$

$\mathrm{Oc}(\mathrm{c}(\mathrm{cc}(\mathrm{c} 1) \mathrm{C}) \mathrm{C}) \mathrm{c} 1 \mathrm{C}$

$\mathrm{O}(\mathrm{c}(\mathrm{c}(\mathrm{ccc} 1) \mathrm{C}) \mathrm{c} 1) \mathrm{C}$

$\mathrm{O}(\mathrm{C}(\mathrm{CCC} 1 \mathrm{C} 2)(\mathrm{C} 2) \mathrm{C}) \mathrm{C} 1(\mathrm{C}) \mathrm{C}$

$\mathrm{N}(\mathrm{C}(=\mathrm{S}) \mathrm{NCCCC}) \mathrm{CCCC}$

$\mathrm{BrCCCC}$

\begin{tabular}{|c|c|c|c|c|}
\hline 2.52 & 2.69 & 0.17 & 2.26 & -0.26 \\
\hline 2.52 & 2.94 & 0.42 & 2.95 & 0.43 \\
\hline 2.53 & 2.13 & -0.40 & 2.25 & -0.28 \\
\hline 2.53 & 2.51 & -0.02 & 2.24 & -0.29 \\
\hline 2.53 & 2.98 & 0.45 & 2.86 & 0.33 \\
\hline 2.54 & 2.46 & -0.08 & 2.50 & -0.04 \\
\hline 2.55 & 2.60 & 0.05 & 2.07 & -0.48 \\
\hline 2.56 & 2.63 & 0.07 & 2.62 & 0.06 \\
\hline 2.57 & 2.31 & -0.26 & 2.57 & 0.00 \\
\hline 2.57 & 2.69 & 0.12 & 2.57 & 0.00 \\
\hline 2.57 & 2.76 & 0.19 & 1.95 & -0.62 \\
\hline 2.58 & 2.55 & -0.03 & 2.54 & -0.04 \\
\hline 2.59 & 2.69 & 0.10 & 2.66 & 0.07 \\
\hline 2.59 & 2.89 & 0.30 & 2.83 & 0.24 \\
\hline 2.60 & 2.23 & -0.37 & 2.41 & -0.19 \\
\hline 2.60 & 2.60 & 0.00 & 2.58 & -0.02 \\
\hline 2.60 & 2.69 & 0.09 & 2.53 & -0.07 \\
\hline 2.61 & 2.69 & 0.08 & 2.56 & -0.05 \\
\hline 2.61 & 2.82 & 0.21 & 2.70 & 0.09 \\
\hline 2.62 & 2.31 & -0.31 & 2.53 & -0.09 \\
\hline 2.62 & 2.36 & -0.26 & 2.58 & -0.04 \\
\hline 2.62 & 2.70 & 0.08 & 2.43 & -0.19 \\
\hline 2.64 & 2.32 & -0.32 & 2.39 & -0.25 \\
\hline 2.64 & 2.56 & -0.08 & 2.37 & -0.27 \\
\hline 2.64 & 2.96 & 0.32 & 2.61 & -0.03 \\
\hline 2.65 & 2.34 & -0.31 & 2.05 & -0.60 \\
\hline 2.65 & 2.68 & 0.03 & 2.92 & 0.27 \\
\hline 2.66 & 2.62 & -0.04 & 2.63 & -0.03 \\
\hline 2.66 & 2.67 & 0.01 & 2.49 & -0.17 \\
\hline 2.67 & 2.54 & -0.13 & 2.75 & 0.08 \\
\hline 2.67 & 2.64 & -0.03 & 2.70 & 0.03 \\
\hline 2.67 & 2.71 & 0.04 & 2.76 & 0.09 \\
\hline 2.67 & 3.15 & 0.48 & 2.72 & 0.05 \\
\hline 2.67 & 3.24 & 0.57 & 2.82 & 0.15 \\
\hline 2.68 & 2.15 & -0.53 & 2.37 & -0.31 \\
\hline 2.68 & 2.52 & -0.16 & 2.24 & -0.44 \\
\hline 2.68 & 2.67 & -0.01 & 2.92 & 0.24 \\
\hline 2.68 & 3.21 & 0.53 & 3.20 & 0.52 \\
\hline 2.69 & 2.37 & -0.32 & 2.74 & 0.05 \\
\hline 2.69 & 2.41 & -0.28 & 2.62 & -0.07 \\
\hline 2.69 & 2.62 & -0.07 & 2.88 & 0.19 \\
\hline 2.69 & 2.85 & 0.16 & 2.83 & 0.14 \\
\hline 2.70 & 2.38 & -0.32 & 2.47 & -0.23 \\
\hline 2.70 & 2.69 & -0.01 & 2.93 & 0.23 \\
\hline 2.70 & 2.79 & 0.09 & 3.09 & 0.39 \\
\hline 2.71 & 3.07 & 0.36 & 2.77 & 0.06 \\
\hline 2.72 & 2.57 & -0.15 & 3.52 & 0.80 \\
\hline 2.72 & 2.66 & -0.06 & 2.06 & -0.66 \\
\hline 2.72 & 3.03 & 0.31 & 2.90 & 0.18 \\
\hline 2.72 & 3.04 & 0.32 & 2.79 & 0.07 \\
\hline 2.73 & 2.11 & -0.62 & 2.36 & -0.37 \\
\hline 2.73 & 2.54 & -0.19 & 2.56 & -0.17 \\
\hline 2.73 & 3.06 & 0.33 & 2.79 & 0.06 \\
\hline 2.73 & 3.15 & 0.42 & 2.72 & -0.01 \\
\hline 2.74 & 2.62 & -0.12 & 2.60 & -0.1 \\
\hline 2.74 & 3.13 & 0.39 & 3.36 & 0.62 \\
\hline 2.75 & 2.57 & -0.18 & 2.62 & -0.13 \\
\hline 2.75 & 2.65 & -0.10 & 2.73 & -0.0 \\
\hline
\end{tabular}


thiophene, 2-bromo-

octanenitrile

pentanoic acid, 2-propyl-

propane, 2-methyl-

pregna-1,4-diene-3,20-dione, 21-(acetyloxy)-9-

fluoro-11,17-dihydroxy-16-methyl-, (11-beta,16beta)-

1-butanone, 1-phenyl-

benzenebutanoic acid, methyl ester

benzenamine, 2,4-dichloro-

1H-pyrrole-2-acetic acid, 1-methyl-5-(4-

methylbenzoyl)-

methanone, diphenyl-, hydrazone

1(3H)-isobenzofuranone, 3-butyl-

$1 \mathrm{H}$-isoindole-1,3(2H)-dione, 3a,4,7,7a-tetrahydro-2-

[(trichloromethyl)thio]-

alpha-glucopyranosiduronic acid, (3,20 )-20-

carboxy-11-oxo-30-norolean-12-en-3-yl 2-o-

glucopyranuronosyl-

cholan-24-oic acid, 3,6,7-trihydroxy-, (3-alpha,5 ,6alpha,7-alpha)-

acetic acid, (2,4-dichlorophenoxy)-

benzenamine, N,N,4-trimethyl-

2H-1,4-benzodiazepin-2-one, 7-chloro-1,3-dihydro-

1-methyl-5-phenyl-

1-hexanamine, 2-ethyl-

ethane, 1,2-dichloro-1,1,2,2-tetrafluoro-

prosta-5,13-dien-1-oic acid, 11,15-dihydroxy-9-oxo-

methane, tetrachloro-

carbamic acid, (5-benzoyl-1h-benzimidazol-2-yl)-,

methyl ester

1-butanamine, N-butyl-

urea, N-(2-chloroethyl)-N'-cyclohexyl-N-nitroso-

benzene, chloro-

propane, 2,2-thiobis-

1-naphthalenol

benzenamine, $\mathrm{N}, \mathrm{N}, 2$-trimethyl-

1H-isoindole-1,3(2H)-dione, 2-

[(trichloromethyl)thio]-

cyclohexanone, 2-methyl-5-(1-methylethenyl)-

2-cyclohexen-1-one, 3-methyl-6-(1-methylethyl)-

benzoic acid, 4-bromo-

cyclohexene

1,5-hexadiene

2-propenoic acid, 2-methyl-, butyl ester

1,3(2H,9bH)-dibenzofurandione, 2,6-diacetyl-7,9-

dihydroxy-8,9b-dimethyl-

pyridine, 4-[(4-nitrophenyl)methyl]-

phenol, 2-(1-methylethyl)-

3,5-pyridinedicarboxylic acid, 1,4-dihydro-2,6dimethyl-4-(3-nitrophenyl)-, ethyl methyl ester butane

propane, 2-iodo-

9H-fluorene-9-methanol

2-octanol

1-octanamine

phenol, 4-(1-methylethyl)-

butane, 1-isothiocyanato-

benzene, (bromomethyl)-

$1 \mathrm{H}$-indene

azacyclotridecan-2-one

phenol, 2-propyl-

benzene, ethenyl-
$\mathrm{C} 1=\mathrm{CC}=\mathrm{C}(\mathrm{Br}) \mathrm{S} 1$

$\mathrm{C}(\# \mathrm{~N}) \mathrm{CCCCCCC}$

$\operatorname{cccc}(\operatorname{CcC}) \mathrm{C}(\mathrm{O})=\mathrm{O}$

$\mathrm{C}(\mathrm{C})(\mathrm{C}) \mathrm{C}$

$\mathrm{CC} 4 \mathrm{CC} 3 \mathrm{C} 2 \mathrm{CCC} 1=\mathrm{CC}(=\mathrm{O}) \mathrm{C}=\mathrm{CC} 1(\mathrm{C}$

) $\mathrm{C} 2(\mathrm{~F}) \mathrm{C}(\mathrm{O}) \mathrm{CC} 3(\mathrm{C}) \mathrm{C} 4(\mathrm{O}) \mathrm{C}(=\mathrm{O}) \mathrm{COC}($ $\mathrm{C})=\mathrm{O}$

$\mathrm{O}=\mathrm{C}(\mathrm{c}(\operatorname{cccc} 1) \mathrm{c} 1) \mathrm{CCC}$

$\operatorname{COC}(=0) \operatorname{CCC} 1 \mathrm{ccccc} 1$

$\mathrm{Nc}(\mathrm{c}(\mathrm{cc}(\mathrm{c} 1) \mathrm{Cl}) \mathrm{Cl}) \mathrm{c} 1$

$\mathrm{Cc} 1 \operatorname{ccc}(\operatorname{cc} 1) \mathrm{C}(=\mathrm{O}) \operatorname{c} 2 \operatorname{ccc}(\mathrm{CC}(\mathrm{O})=\mathrm{O}) \mathrm{n}$

2C

$\mathrm{N}(\mathrm{N})=\mathrm{C}(\mathrm{c}(\mathrm{cccc} 1) \mathrm{c} 1) \mathrm{c}(\mathrm{cccc} 2) \mathrm{c} 2$

$\mathrm{O}=\mathrm{C}(\mathrm{OC}(\mathrm{c} 1 \mathrm{cccc} 2) \mathrm{CCCC}) \mathrm{c} 12$

$\mathrm{O}=\mathrm{C}(\mathrm{N}(\mathrm{SC}(\mathrm{Cl})$

$(\mathrm{Cl}) \mathrm{Cl}) \mathrm{C}(=\mathrm{O}) \mathrm{C} 1 \mathrm{CC}=\mathrm{CC} 2) \mathrm{C} 12$

$\mathrm{O} 1 \mathrm{C}(\mathrm{C}(=\mathrm{O}) \mathrm{O}) \mathrm{C}(\mathrm{O}) \mathrm{C}(\mathrm{O}) \mathrm{C}(\mathrm{O}) \mathrm{C} 1 \mathrm{OC} 2$

$\mathrm{C}(\mathrm{O}) \mathrm{C}(\mathrm{O}) \mathrm{C}(\mathrm{C}(=\mathrm{O}) \mathrm{O}) \mathrm{OC} 2 \mathrm{OC} 3 \mathrm{C}(\mathrm{C})$

(C) $44 \mathrm{CCC} 5(\mathrm{C}) \mathrm{C} 6(\mathrm{C}) \mathrm{CCC} 7(\mathrm{C}) \mathrm{CCC}($

C)

$(\mathrm{C}(=\mathrm{O}) \mathrm{O}) \mathrm{CC} 7 \mathrm{C} 6=\mathrm{CC}(=\mathrm{O}) \mathrm{C} 5 \mathrm{C} 4(\mathrm{C}) \mathrm{C}$

$\mathrm{C} 3$

$\mathrm{C} 1 \mathrm{C}(\mathrm{O}) \mathrm{CC} 2 \mathrm{C}(\mathrm{O}) \mathrm{C}(\mathrm{O}) \mathrm{C} 3 \mathrm{C} 4 \mathrm{CCC}(\mathrm{C}(\mathrm{C}$

) $\mathrm{CCC}(=\mathrm{O}) \mathrm{O}) \mathrm{C} 4(\mathrm{C}) \mathrm{CCC} 3 \mathrm{C} 2(\mathrm{C}) \mathrm{C} 1$

$\mathrm{O}=\mathrm{C}(\mathrm{O}) \mathrm{COc}(\mathrm{c}(\mathrm{cc}(\mathrm{c} 1) \mathrm{Cl}) \mathrm{Cl}) \mathrm{c} 1$

$\mathrm{N}(\mathrm{c}(\operatorname{ccc}(\mathrm{c} 1) \mathrm{C}) \mathrm{c} 1)(\mathrm{C}) \mathrm{C}$

$\mathrm{O}=\mathrm{C}(\mathrm{N}(\mathrm{c}(\mathrm{c}(\mathrm{cc}(\mathrm{c} 1) \mathrm{Cl}) \mathrm{C}(=\mathrm{N} 2) \mathrm{c}(\operatorname{cccc} 3) \mathrm{c}$

3)c1)C)C2

$\mathrm{NCC}(\mathrm{CCCC}) \mathrm{CC}$

$\mathrm{FC}(\mathrm{F})(\mathrm{C}(\mathrm{F})(\mathrm{F}) \mathrm{Cl}) \mathrm{Cl}$

$\operatorname{CCCCCC}(\mathrm{O}) \mathrm{C}=\mathrm{CC} 1 \mathrm{C}(\mathrm{O}) \mathrm{CC}(=\mathrm{O}) \mathrm{C} 1$

$\mathrm{CC}=\operatorname{CcccC}(=0) \mathrm{O}$

$\mathrm{C}(\mathrm{Cl})(\mathrm{Cl})(\mathrm{Cl}) \mathrm{Cl}$

c1c $(\mathrm{C}(=\mathrm{O}) \mathrm{c} 3 \mathrm{ccccc} 3) \mathrm{cc} 2 \mathrm{nc}(\mathrm{NC}(=\mathrm{O}) \mathrm{O}$

$\mathrm{C}) \mathrm{n}(\mathrm{H}) \mathrm{c} 2 \mathrm{c} 1$

$\mathrm{N}(\mathrm{CCCC}) \mathrm{CCCC}$

$\mathrm{CICCN}(\mathrm{N}=\mathrm{O}) \mathrm{C}(=\mathrm{O}) \mathrm{NC} 1 \mathrm{CCCCC} 1$

$\mathrm{c}(\mathrm{cccc} 1)(\mathrm{c} 1) \mathrm{Cl}$

$\mathrm{S}(\mathrm{C}(\mathrm{C}) \mathrm{C}) \mathrm{C}(\mathrm{C}) \mathrm{C}$

$\mathrm{Oc}(\mathrm{c}(\mathrm{c}(\mathrm{ccc} 1) \mathrm{cc} 2) \mathrm{c} 1) \mathrm{c} 2$

$\mathrm{N}(\mathrm{c}(\mathrm{c}(\operatorname{ccc} 1) \mathrm{C}) \mathrm{c} 1)(\mathrm{C}) \mathrm{C}$

$\mathrm{O}=\mathrm{C}(\mathrm{N}(\mathrm{SC}(\mathrm{Cl})$

$(\mathrm{Cl}) \mathrm{Cl}) \mathrm{C}(=\mathrm{O}) \mathrm{c} 1 \mathrm{cccc} 2) \mathrm{c} 12$

$\mathrm{O}=\mathrm{C} 1 \mathrm{CC}(\mathrm{C}(=\mathrm{C}) \mathrm{C}) \mathrm{CCC} 1 \mathrm{C}$

$\mathrm{O}=\mathrm{C}(\mathrm{C}=\mathrm{C}(\mathrm{CC} 1) \mathrm{C}) \mathrm{C} 1 \mathrm{C}(\mathrm{C}) \mathrm{C}$

$\mathrm{O}=\mathrm{C}(\mathrm{O}) \mathrm{c}(\operatorname{ccc}(\mathrm{c} 1) \mathrm{Br}) \mathrm{c} 1$

$\mathrm{C}(=\mathrm{CCCC} 1) \mathrm{C} 1$

$\mathrm{C}(=\mathrm{C}) \mathrm{CCC}=\mathrm{C}$

$\mathrm{O}=\mathrm{C}(\mathrm{OCCCC}) \mathrm{C}(=\mathrm{C}) \mathrm{C}$

$\mathrm{O}=\mathrm{C}(\mathrm{c}(\mathrm{c}(\mathrm{OC}(\mathrm{C} 1(\mathrm{C}(=\mathrm{O}) \mathrm{C}(\mathrm{C} 2(=\mathrm{O})) \mathrm{C}(=$

$\mathrm{O}) \mathrm{C}) \mathrm{C})=\mathrm{C} 2) \mathrm{c} 1 \mathrm{c}(\mathrm{O}) \mathrm{c} 3 \mathrm{C}) \mathrm{c} 3 \mathrm{O}) \mathrm{C}$

$\mathrm{N}(=\mathrm{O})(=\mathrm{O}) \mathrm{c}(\operatorname{ccc}(\mathrm{c} 1) \mathrm{Cc}(\mathrm{ccnc} 2) \mathrm{c} 2) \mathrm{c} 1$

$\mathrm{Oc}(\mathrm{c}(\operatorname{ccc} 1) \mathrm{C}(\mathrm{C}) \mathrm{C}) \mathrm{c} 1$

$\operatorname{c1} \operatorname{ccc}(\mathrm{N}(=\mathrm{O})=\mathrm{O}) \operatorname{cc} 1 \mathrm{C} 2 \mathrm{C}(\mathrm{C}(=\mathrm{O}) \mathrm{OC})=$

$\mathrm{C}(\mathrm{C}) \mathrm{NC}(\mathrm{C})=\mathrm{C} 2 \mathrm{C}(=\mathrm{O}) \mathrm{OCC}$

$\mathrm{C}(\mathrm{CC}) \mathrm{C}$

$\mathrm{C}(\mathrm{C})(\mathrm{C}) \mathrm{I}$

OCC (c(c(c1cccc2)ccc3)c3)c12

$\mathrm{OC}(\mathrm{CCCCCC}) \mathrm{C}$

$\mathrm{NCCCCCCCC}$

$\mathrm{Oc}(\operatorname{ccc}(\mathrm{c} 1) \mathrm{C}(\mathrm{C}) \mathrm{C}) \mathrm{c} 1$

$\mathrm{N}(=\mathrm{C}=\mathrm{S}) \mathrm{CCCC}$

$\mathrm{BrCc}(\mathrm{cccc} 1) \mathrm{c} 1$

$\mathrm{c}(\mathrm{c}(\mathrm{C}=\mathrm{C} 1) \mathrm{ccc} 2)(\mathrm{c} 2) \mathrm{C} 1$

$\mathrm{O}=\mathrm{C}(\mathrm{NCCCCCCCCCC} 1) \mathrm{C} 1$

Oc(c(ccc1)CCC) 1

$\mathrm{c}(\mathrm{cccc} 1)(\mathrm{c} 1) \mathrm{C}=\mathrm{C}$

2.80

2.81

2.82

2.82

2.82

2.83

2.83

2.84

2.84

2.85

2.85

2.85

$\begin{array}{lllll}2.75 & 2.70 & -0.05 & 2.60 & -0.15 \\ 2.75 & 2.80 & 0.05 & 2.76 & 0.01 \\ 2.75 & 2.96 & 0.21 & 2.54 & -0.21 \\ 2.76 & 2.23 & -0.53 & 2.16 & -0.60 \\ 2.77 & 2.46 & -0.31 & 2.60 & -0.17 \\ & & & & \\ & & & & \\ 2.77 & 2.66 & -0.11 & 2.55 & -0.22 \\ 2.77 & 3.06 & 0.29 & 2.89 & 0.12 \\ 2.78 & 2.37 & -0.41 & 2.73 & -0.05 \\ 2.79 & 2.56 & -0.23 & 2.81 & 0.02 \\ & & & & \\ 2.79 & 3.31 & 0.52 & 3.23 & 0.44 \\ 2.80 & 2.61 & -0.19 & 3.00 & 0.20 \\ 2.80 & 2.74 & -0.06 & 3.00 & 0.20 \\ & & & & \\ 2.80 & 3.19 & 0.39 & 2.78 & -0.02\end{array}$

3.52

0.72

2.16

$-0.64$

2.62

$-0.19$

2.82

2.58

0.01

2.70

$-0.12$

2.63

$-0.19$

2.73

$-0.09$

3.10

2.78

$-0.04$

2.57

3.31

0.28

$-0.25$

0.49

2.44

$-0.39$

2.64

$-0.19$

2.71

$-0.12$

2.67

$-0.16$

2.77

$-0.06$

2.71

$-0.12$

0.13

$-0.20$

2.78

2.74

$-0.10$

$-0.16$

$-0.13$

2.72

2.84

$-0.01$

2.79

2.56

2.92

0.01

0.22

2.39

2.86
3.07

2.76

2.96

$-0.10$

0.10

0.15

$-0.13$

$-0.10$

2.47

2.43

2.77

3.05

2.59

2.77

0.00

2.74

2.88

2.97

0.09

2.74
2.78

$0.11 \quad 3.21$

2.31

$-0.58$

$-0.39$

$-0.20$

$-0.17$

$-0.10$

0.07

$-0.15$

$-0.04$

0.33

0.69

0.11

$-0.06$ 
benzene, 1,3-dimethyl-2-nitrobenzoic acid, 2-hydroxy-, ethyl ester 1-naphthalenol, 5,6,7,8-tetrahydro19-norpregn-4-en-20-yn-3-one, 17-hydroxy-, (17alpha)-

7-oxabicyclo[2.2.1]heptane, 1-methyl-4-(1methylethyl)-

1,6-octadien-3-ol, 3,7-dimethyl-

bicyclo[3.1.1] hept-2-ene-2-carboxaldehyde, 6,6dimethyl-

ethanone, 1-[4-(1-methylethyl)phenyl]-

benzoic acid, 2-hydroxy-4-methyl-

2-propenoic acid, 3-phenyl-, ethyl ester

benzene, bromo-

cyclopentane

1-octanol

urea, N,N-diphenyl-

9,10-anthracenedione, 1,4-diamino-

benzoic acid, propyl ester

4H-1-benzopyran-4-one, 5,7-dihydroxy-2-(4hydroxyphenyl)-

benzoic acid, 4-iodo-

phenol, 4,4-(3H-2,1-benzoxathiol-3-ylidene)bis-,

S,S-dioxide

phenol, 2-methoxy-4-(1-propenyl)-

benzoic acid, 4-hydroxy-, propyl ester

cyclohexanone, 5-methyl-2-(1-methylethyl)-

benzene, 1-bromo-4-methoxy-

octanoic acid

benzenamine, 4-butyl-

2-naphthalenecarboxylic acid, 3-hydroxy-

1,3-benzenedicarbonitrile, 2,4,5,6-tetrachloroerythromycin
$\mathrm{N}(=\mathrm{O})(=\mathrm{O}) \mathrm{c}(\mathrm{c}(\mathrm{ccc} 1) \mathrm{C}) \mathrm{c} 1 \mathrm{C}$
$\mathrm{O}=\mathrm{C}(\mathrm{OCC}) \mathrm{c}(\mathrm{c}(\mathrm{O}) \mathrm{ccc} 1) \mathrm{c} 1$
$\mathrm{c} 1 \mathrm{c}(\mathrm{O}) \mathrm{c} 2 \mathrm{CCCC} 2 \mathrm{cc} 1$
$\mathrm{CC} 34 \mathrm{CCC} 1 \mathrm{C}(\mathrm{CCC} 2=\mathrm{CC}(=\mathrm{O}) \mathrm{CCC} 12$
) $\mathrm{C} 3 \mathrm{CCC} 4(\mathrm{O}) \mathrm{CHC}$
$\mathrm{O}(\mathrm{C}(\mathrm{CC} 1)(\mathrm{CC} 2) \mathrm{C}(\mathrm{C}) \mathrm{C}) \mathrm{C} 12 \mathrm{C}$

2H-1,2-benzothiazine-3-carboxamide, 4-hydroxy-2methyl-N-2-pyridinyl-, 1,1-dioxide

phenol, 2,5-dichloro-

phenol, 2,4-dichloro-

cyclohexanone, 5-methyl-2-(1-methylethylidene)-

phenol, 4-(1-methylpropyl)-

cholan-24-oic acid, 3,6-dihydroxy-, (3-alpha,5 ,6alpha)-

benzeneacetic acid, alpha-phenyl-

[1,1-biphenyl]-2-ol

benzene, (2-bromoethyl)-

cyclohexanol, 4-(1,1-dimethylethyl)-

phenol, 4-chloro-3-methyl-

2,4-oxazolidinedione, 3-(3,5-dichlorophenyl)-5ethenyl-5-methyl-

propane, 2,2-dimethyl-

benzene, 1,2-dichloro-4,5-dimethoxy-

benzene, 1,2-dimethyl-

benzene, 1,2-dichloro-4-nitro-

2-cyclohexen-1-ol, 2-methyl-5-(1-methylethenyl)propanoic acid, 2-(4-chloro-2-methylphenoxy)-

benzenemethanamine, N-phenyl-

benzenamine, $\mathrm{N}$-nitroso- $\mathrm{N}$-phenyl-

1-cyclohexene-1-carboxaldehyde, 4-(1-

methylethenyl)-

2-nonanone

benzene, ethyl-

benzene, 1,4-dimethyl-

bicyclo[3.1.1]hept-3-en-2-ol, 4,6,6-trimethyl-

ethane, 1,1,2-trichloro-1,2,2-trifluoro-
$\mathrm{O}(\mathrm{C}(\mathrm{CC} 1)(\mathrm{CC} 2) \mathrm{C}(\mathrm{C}) \mathrm{C}) \mathrm{C} 12 \mathrm{C}$

$\mathrm{OC}(\mathrm{C}=\mathrm{C})(\mathrm{CCC}=\mathrm{C}(\mathrm{C}) \mathrm{C}) \mathrm{C}$
$\mathrm{O}=\mathrm{CC}(\mathrm{C}(\mathrm{CC} 1 \mathrm{C} 2) \mathrm{C} 1(\mathrm{C}) \mathrm{C})=\mathrm{C} 2$

$\mathrm{CC}(=\mathrm{O}) \mathrm{c} 1 \mathrm{ccc}(\mathrm{C}(\mathrm{C}) \mathrm{C}) \mathrm{cc} 1$

$\mathrm{Cc} 1 \mathrm{ccc}(\mathrm{C}(=\mathrm{O})(\mathrm{O})) \mathrm{c}(\mathrm{O}) \mathrm{c} 1$
$\mathrm{O}=\mathrm{C}(\mathrm{OCC}) \mathrm{C}=\mathrm{Cc}(\mathrm{cccc} 1) \mathrm{c} 1$

$\mathrm{c}(\mathrm{cccc} 1)(\mathrm{c} 1) \mathrm{Br} \quad 2.99$

$\mathrm{C}(\mathrm{CCC} 1) \mathrm{C} 1$

OCCCCCCCC 3.00

$\mathrm{O}=\mathrm{C}(\mathrm{Nc}(\operatorname{cccc} 1) \mathrm{c} 1) \mathrm{Nc}(\operatorname{cccc} 2) \mathrm{c} 2$

$\mathrm{O}=\mathrm{C}(\mathrm{c}(\mathrm{c}(\mathrm{C}(=\mathrm{O}) \mathrm{c} 1 \mathrm{c}(\mathrm{N}) \mathrm{ccc} 2 \mathrm{~N}) \mathrm{ccc} 3) \mathrm{c} 3)$

$\mathrm{c} 12$

$\mathrm{O}=\mathrm{C}(\mathrm{OCCC}) \mathrm{c}(\mathrm{cccc} 1) \mathrm{c} 1$
$\mathrm{Oc} 1 \mathrm{cc}(\mathrm{O}) \mathrm{c} 2 \mathrm{C}(=\mathrm{O}) \mathrm{C}=\mathrm{C}(\mathrm{c} 3 \mathrm{ccc}(\mathrm{O}) \mathrm{cc} 3)$

Oc2c1

$\mathrm{O}=\mathrm{C}(\mathrm{O}) \mathrm{c}(\operatorname{ccc}(\mathrm{c} 1) \mathrm{l}) \mathrm{c} 1$

$\mathrm{O}=\mathrm{S}(=\mathrm{O})(\mathrm{OC}(\mathrm{c} 1 \mathrm{cccc} 2)$

$(\mathrm{c}(\operatorname{ccc}(\mathrm{O}) \mathrm{c} 3) \mathrm{c} 3) \mathrm{c}(\operatorname{ccc}(\mathrm{O}) \mathrm{c} 4) \mathrm{c} 4) \mathrm{c} 12$

$\mathrm{O}(\mathrm{c}(\mathrm{c}(\mathrm{O}) \operatorname{ccc} 1 \mathrm{C}=\mathrm{CC}) \mathrm{c} 1) \mathrm{C}$

$\mathrm{O}=\mathrm{C}(\mathrm{OCCC}) \mathrm{c}(\operatorname{ccc}(\mathrm{O}) \mathrm{c} 1) \mathrm{c} 1$

$\mathrm{O}=\mathrm{C}(\mathrm{C}(\mathrm{CCC} 1 \mathrm{C}) \mathrm{C}(\mathrm{C}) \mathrm{C}) \mathrm{C} 1$

$\mathrm{O}(\mathrm{c}(\mathrm{ccc}(\mathrm{c} 1) \mathrm{Br}) \mathrm{c} 1) \mathrm{C}$

$\mathrm{O}=\mathrm{C}(\mathrm{O}) \mathrm{CCCCCCC}$

$\mathrm{Nc}(\operatorname{ccc}(\mathrm{c} 1) \mathrm{CCCC}) \mathrm{c} 1$

$\mathrm{O}=\mathrm{C}(\mathrm{O}) \mathrm{c}(\mathrm{c}(\mathrm{O}) \mathrm{cc}(\mathrm{c} 1 \mathrm{ccc} 2) \mathrm{c} 2) \mathrm{c} 1$

$\mathrm{N \# Cc(c(c(c(c1C \# N)Cl)Cl)Cl)c1Cl}$

$\operatorname{CCC} 3 \mathrm{OC}(=\mathrm{O}) \mathrm{C}(\mathrm{C}) \mathrm{C}(\mathrm{OC} 1 \mathrm{CC}(\mathrm{C})$

$(\mathrm{OC}) \mathrm{C}(\mathrm{O}) \mathrm{C}(\mathrm{C}) \mathrm{O} 1) \mathrm{C}(\mathrm{C}) \mathrm{C}(\mathrm{OC} 2 \mathrm{OC}(\mathrm{C})$

$\mathrm{CC}(\mathrm{C} 2 \mathrm{O}) \mathrm{N}(\mathrm{C}) \mathrm{C}) \mathrm{C}(\mathrm{C})$

(O)CC $(\mathrm{C}) \mathrm{C}(=\mathrm{O}) \mathrm{C}(\mathrm{C}) \mathrm{C}(\mathrm{O}) \mathrm{C} 3(\mathrm{C}) \mathrm{O}$

$\begin{array}{lllll}2.95 & 2.91 & -0.04 & 2.76 & -0.19 \\ 2.95 & 3.09 & 0.14 & 2.76 & -0.19 \\ 2.95 & 3.48 & 0.53 & 2.83 & -0.12 \\ 2.97 & 2.99 & 0.02 & 2.72 & -0.25 \\ & & & & \\ 2.97 & 3.13 & 0.16 & 2.31 & -0.66 \\ & & & & \\ 2.97 & 3.38 & 0.41 & 2.68 & -0.29 \\ 2.98 & 2.78 & -0.20 & 3.03 & 0.05 \\ & & & & \\ 2.98 & 3.13 & 0.15 & 3.12 & 0.14 \\ 2.99 & 2.79 & -0.20 & 2.39 & -0.60 \\ 2.99 & 2.85 & -0.14 & 3.10 & 0.11 \\ 2.99 & 2.88 & -0.11 & 2.65 & -0.34 \\ 3.00 & 2.68 & -0.32 & 2.88 & -0.12 \\ 3.00 & 2.81 & -0.19 & 3.21 & 0.21 \\ 3.00 & 2.97 & -0.03 & 3.10 & 0.10 \\ 3.00 & 3.16 & 0.16 & 3.00 & 0.00 \\ & & & & \\ 3.01 & 2.81 & -0.20 & 2.92 & -0.09 \\ 3.02 & 2.84 & -0.18 & 3.07 & 0.05 \\ & & & & \\ 3.02 & 3.04 & 0.02 & 2.55 & -0.47 \\ 3.02 & 3.21 & 0.19 & 3.15 & 0.13 \\ 3.04 & 2.65 & -0.39 & 2.79 & -0.25 \\ 3.04 & 2.98 & -0.06 & 3.28 & 0.24 \\ 3.05 & 2.87 & -0.18 & 2.65 & -0.40 \\ 3.05 & 2.96 & -0.09 & 2.93 & -0.12 \\ 3.05 & 3.03 & -0.02 & 2.92 & -0.13 \\ 3.05 & 3.10 & 0.05 & 2.95 & -0.10 \\ 3.05 & 3.42 & 0.37 & 3.30 & 0.25 \\ 3.05 & 3.66 & 0.61 & 3.98 & 0.93 \\ 3.06 & 2.48 & -0.58 & 2.37 & -0.69\end{array}$

CN2C $(=\mathrm{C}(\mathrm{O}) \mathrm{c} 1 \operatorname{ccccc} 1 \mathrm{~S} 2(=\mathrm{O})=\mathrm{O}) \mathrm{C}(=$

) Nc3ccccn 3

$\mathrm{Oc}(\mathrm{c}(\operatorname{ccc} 1 \mathrm{Cl}) \mathrm{Cl}) \mathrm{c} 1$

$\mathrm{Oc}(\mathrm{c}(\mathrm{cc}(\mathrm{c} 1) \mathrm{Cl}) \mathrm{Cl}) \mathrm{c} 1$

$\mathrm{O}=\mathrm{C}(\mathrm{C}(=\mathrm{C}(\mathrm{C}) \mathrm{C}) \mathrm{CCC} 1 \mathrm{C}) \mathrm{C} 1$

$\mathrm{Oc}(\operatorname{ccc}(\mathrm{c} 1) \mathrm{C}(\mathrm{CC}) \mathrm{C}) \mathrm{c} 1$

$\mathrm{O}=\mathrm{C}(\mathrm{CCC}(\mathrm{C} 1 \mathrm{C} 2(\mathrm{CCC} 3 \mathrm{C} 4(\mathrm{C}) \mathrm{C}(\mathrm{CC} 3$

$\mathrm{C} 2 \mathrm{CC} 1) \mathrm{O})(\mathrm{CC}(\mathrm{CC} 4) \mathrm{O})) \mathrm{C}) \mathrm{C}) \mathrm{C}) \mathrm{O}$

$\mathrm{O}=\mathrm{C}(\mathrm{O}) \mathrm{C}(\mathrm{c}(\mathrm{cccc} 1) \mathrm{c} 1) \mathrm{c}(\mathrm{cccc} 2) \mathrm{c} 2$

Oc(c(c) (cccc1)c1) ccc2)c2

$\mathrm{BrCCc}(\operatorname{cccc} 1) \mathrm{c} 1$

$\mathrm{OC}(\operatorname{CCC}(\mathrm{C}(\mathrm{C})(\mathrm{C}) \mathrm{C}) \mathrm{C} 1) \mathrm{C} 1$

$\mathrm{Oc}(\mathrm{ccc}(\mathrm{c} 1 \mathrm{C}) \mathrm{Cl}) \mathrm{c} 1$

$\mathrm{c} 1 \mathrm{c}(\mathrm{Cl}) \mathrm{cc}(\mathrm{Cl}) \operatorname{cc} 1 \mathrm{~N} 2 \mathrm{C}(=\mathrm{O}) \mathrm{C}(\mathrm{C})$

$(\mathrm{C}=\mathrm{C}) \mathrm{OC} 2(=\mathrm{O})$

$\mathrm{C}(\mathrm{C})(\mathrm{C})(\mathrm{C}) \mathrm{C}$

COc1c $(\mathrm{OC}) \mathrm{cc}(\mathrm{Cl}) \mathrm{c}(\mathrm{Cl}) \mathrm{c} 1$

$\mathrm{c}(\mathrm{c}(\mathrm{ccc} 1) \mathrm{C})(\mathrm{c} 1) \mathrm{C}$

$\mathrm{N}(=\mathrm{O})(=\mathrm{O}) \mathrm{c}(\operatorname{ccc}(\mathrm{c} 1 \mathrm{Cl}) \mathrm{Cl}) \mathrm{c} 1$

$\mathrm{C} 1(\mathrm{C})=\mathrm{CCC}(\mathrm{C}(\mathrm{C})=\mathrm{C}) \mathrm{CC} 10$

$\mathrm{O}=\mathrm{C}(\mathrm{O}) \mathrm{C}(\mathrm{Oc}(\mathrm{c}(\mathrm{cc}(\mathrm{c} 1) \mathrm{Cl}) \mathrm{C}) \mathrm{c} 1) \mathrm{C}$

$\mathrm{N}$ (c(cccc1)c1)Cc(cccc2)c2

$\mathrm{O}=\mathrm{NN}(\mathrm{c}(\operatorname{cccc} 1) \mathrm{c} 1) \mathrm{c}(\mathrm{cccc} 2) \mathrm{c} 2$

$\mathrm{O}=\mathrm{CC}(=\operatorname{CcC}(\mathrm{C}(=\mathrm{C}) \mathrm{C}) \mathrm{C} 1) \mathrm{C} 1$

3.06

2.58

$-0.48$

2.20

$-0.86$

2.80

$-0.26$

3.13

$-0.26$

3.14

3.20

0.12

2.36

3.35

5.06

1.98

2.98

$-0.03$

3.06

3.28

3.37

3.42

2.70

3.03

0.19

2.79

3.21

3.28

0.33

$-0.40$

3.29

2.74

2.81

2.69

$-0.42$

2.95

3.34

2.93
3.09

3.10

3.29

2.94

3.03

3.16

3.34

$\mathrm{O}=\mathrm{C}(\operatorname{cccccc}) \mathrm{C}$

$\mathrm{c}$ (cccc1)(c1)CC

$\mathrm{c}(\operatorname{ccc}(\mathrm{c} 1) \mathrm{C})(\mathrm{c} 1) \mathrm{C}$

$\mathrm{C} 1(\mathrm{C})(\mathrm{C}) \mathrm{C} 2 \mathrm{C}(\mathrm{O}) \mathrm{C}=\mathrm{C}(\mathrm{C} 1 \mathrm{C} 2) \mathrm{C}$

$\mathrm{FC}(\mathrm{F})(\mathrm{C}(\mathrm{F})(\mathrm{Cl}) \mathrm{Cl}) \mathrm{Cl}$ 
3,5-pyrazolidinedione, 4-butyl-1,2-diphenyl-

1,5-cyclooctadiene

bicyclo[2.2.1]heptan-2-ol, 1,3,3-trimethyl1-cyclohexene-1-methanol, 4-(1-methylethenyl)3-cyclohexene-1-ethanol, 4-methyl--methylenebenzoic acid, 1-methylethyl ester

2-naphthaleneacetic acid, 6-methoxy-alpha-methylmethanone, diphenyl-

pregna-1,4-diene-3,20-dione, 21-(acetyloxy)-6,9difluoro-11-hydroxy-16,17-[(1-

methylethylidene)bis(oxy)]-, (6-alpha,11-beta,16alpha)-

naphthalene, 1-nitro-

cyclohexanol, 5-methyl-2-(1-methylethyl)-

phenol, 4-propyl-

benzene, 1,3-dimethyl-

[1,1-biphenyl]-4-ol

azulene

7-oxabicyclo[4.1.0]heptane, 1-methyl-4-(1-

methylethenyl)-

cyclohexane, bromo-

prost-13-en-1-oic acid, 11,15-dihydroxy-9-oxo-

butane, 1,1-oxybis-

cyclohexanol, 2-methyl-5-(1-methylethenyl)-

bicyclo[3.1.1] hept-2-ene-2-methanol, 6,6-dimethylethane, pentachloro-

androst-5-en-17-one, 3-hydroxy-, (3b)-

[1,1-biphenyl]-3-ol

1,2-benzenedicarboxylic acid, di-2-propenyl ester

benzene, iodo-

phenol, 4,5-dichloro-2-methoxy-

3-cyclohexen-1-ol, 4-methyl-1-(1-methylethyl)butanoic acid, 3-methyl-, phenylmethyl ester ethanamine, 2-(diphenylmethoxy)-N,N-dimethylphenol, 4-chloro-3,5-dimethyl-

benzamide, 2-hydroxy-N-phenyl-

phenol, 2-(1-methylpropyl)-

1,2-benzenedicarboxylic acid, dipropyl ester

carbonic acid, diphenyl ester

benzene, isothiocyanato-

terpineol

thiocyanic acid, (2-benzothiazolylthio)methyl ester

naphthalene

phenol, 5-methyl-2-(1-methylethyl)-

benzenamine, $\mathrm{N}, \mathrm{N}$-diethyl-

phenol, 2-(1,1-dimethylethyl)-

phenol, 4-(1,1-dimethylethyl)-

benzene, 1,1-[oxybis(methylene)]bis-

androst-4-en-3-one, 17-hydroxy-, (17 )-

phenol, 4,4-(1-methylethylidene)bis-

phenol, 4,4-thiobis-

propanamide, 2-methyl-N-[4-nitro-3-

(trifluoromethyl)phenyl]-

phenol, 4-phenoxy-

cyclopentane, methyl-

pentane, 1-bromo-

ethanedione, diphenyl-

pentane

1-hexene

9,10-anthracenedione

ethene, tetrachloro-

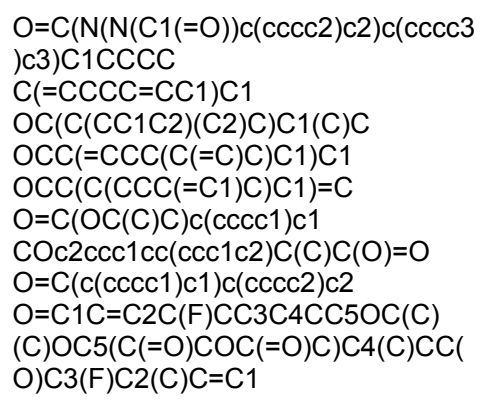

3.35

3.37

3.37

3.38

3.39

3.39

3.39

3.40

$\begin{array}{ccc}0.36 & 2.81 & -0.35 \\ & & \\ 0.57 & 3.58 & 0.42 \\ -0.32 & 2.30 & -0.87 \\ 0.19 & 2.50 & -0.67 \\ 0.19 & 2.55 & -0.62 \\ -0.44 & 2.72 & -0.46 \\ -0.08 & 3.29 & 0.11 \\ -0.03 & 3.03 & -0.15 \\ -0.42 & 2.93 & -0.26\end{array}$

2.99

$-0.20$

0.19

3.20

2.68

3.01

3.15

3.24

0.18

0.23

3.51

2.81

$0.25 \quad 3.63$

$0.53 \quad 3.04$

$-0.20$

0.16

$-0.42$

$-0.11$

$-0.25$

3.04

2.70

2.71

3.21

3.53

0.05

3.19

3.28

3.36

0.13

2.71

3.16

$-0.09$

3.00

3.07

0.07

0.22

$-0.16$

$-0.02$

0.03

0.19

0.36

$-0.07$

0.05

0.05

$-0.18$

$-0.13$

0.22

$-0.16$

0.11

0.11

0.17

$-0.05$

0.32

$0.32 \quad 3.81$

$\begin{array}{ll}-0.01 & 3.73 \\ 0.16 & 2.55\end{array}$

3.51
3.57

3.10

3.14

3.56

2.80

3.15

3.34

2.97
0.01

$-0.51$

$-0.19$

$-0.05$

0.04

0.31

$-0.39$

0.43

$-0.16$

$-0.17$

$-0.51$

$-0.51$

$-0.01$

0.30

$-0.04$

$-0.52$

$-0.25$

$-0.19$

$-0.45$

0.09

0.17

$-0.13$

$-0.62$

$-0.13$

0.27

0.07

$-0.20$

$-0.11$

$-0.01$

0.03

$-0.14$

0.12

0.04

0.16

0.11

$-0.33$

0.49

0.39

$-0.80$

$-0.03$

$-0.22$

$-0.10$

$-0.42$

0.02

$-0.01$

$-0.56$

$-0.27$ 
3-pyridinecarbonitrile, 5-[(4-chloro-2nitrophenyl)azo]-1-ethyl-1,2-dihydro-6-hydroxy-4methyl-2-oxo-

benzenamine, 4-(phenylazo)-

pyridine, 2-chloro-6-(trichloromethyl)-

2-piperidinecarboxamide, 1-butyl-N-(2,6-

dimethylphenyl)-

methane, tetrabromo-

butane, 2,3-dimethyl-

benzene, 1-chloro-2-methyl-

benzene, 1-bromo-4-methyl-

nonanoic acid

benzene, 1,3,5-trimethyl-

benzene, 1,2-dichloro-

cyclohexane

benzene, 1,4-dichloro-

cinchonan-9-ol, 6-methoxy-

1,3-benzenediol, 4-hexyl-

1-naphthalenamine, N,N-dimethyl-

peroxide, dibenzoyl

naphthalene, 2-methoxy-

2,6-octadien-1-ol, 3,7-dimethyl-

benzene, (2-isothiocyanatoethyl)-

benzene, (1-methylethenyl)-

phenol, 2-methyl-5-(1-methylethyl)-

naphthalene, 1,2,3,4-tetrahydro-

pregna-1,4-diene-3,20-dione, 21-chloro-9-fluoro-11-

hydroxy-16-methyl-17-(1-oxopropoxy)-, (11-

beta, 16-beta)-

9,10-anthracenedione, 1,4-diamino-2-methoxy-

benzenamine, $\mathrm{N}$-phenyl-

cholan-24-oic acid, 3,12-dihydroxy-, (3-alpha,5 ,12alpha)-

3-pyridinecarboxylic acid, hexyl ester bicyclo[2.2.1]heptan-2-one, 1,3,3-trimethyl-

$10 \mathrm{H}$-phenothiazine-2-carbonitrile, 10-[3-(4-hydroxy1-piperidinyl)propyl]-

benzene, 1,3-dichloro-

benzene, 1-ethyl-2-methyl-

phosphoric acid, 2-chloro-1-(2,4,5-

trichlorophenyl)ethenyl dimethyl ester

phenol, 2-(1-methylpropyl)-4,6-dinitro-

benzoic acid, 4-hydroxy-, phenylmethyl ester

2,5-pyridinedicarboxylic acid, dipropyl ester

benzoic acid, 4-hydroxy-, butyl ester

1,2-benzenediol, 3,4,6-trichloro-

pentane, 3-methyl-

pregna-1,4-diene-3,20-dione, 9-fluoro-11,21dihydroxy-16-methyl-17-[(1-oxopentyl)oxy]-, (11-

beta, 16-beta)-

cyclohexane, methyl-

benzene, ethylmethyl-

benzene, 1,2,4-trimethyl-

ethanol, 2-(octylthio)-

benzene, 1,2-dibromo-

phenol, 4-butyl-

2-propanol, 1,3-dichloro-, phosphate (3:1)

benzene, (1-methylethyl)-

benzene, 1,2,3-trimethyl-

$9(10 \mathrm{H})$-anthracenone

benzenemethanol, alpha,alpha-diphenyl-

\begin{tabular}{|c|c|c|c|c|c|}
\hline $\begin{array}{l}\mathrm{N}(=\mathrm{O}) \\
(=\mathrm{O}) \mathrm{c} 1 \mathrm{cc}(\mathrm{Cl}) \operatorname{ccc} 1 \mathrm{~N}=\mathrm{NC} 2=\mathrm{C}(\mathrm{O}) \mathrm{N}(\mathrm{CC}) \\
\mathrm{C}(=\mathrm{O}) \mathrm{C}(\mathrm{C}(\# \mathrm{~N}))=\mathrm{C} 2 \mathrm{C}\end{array}$ & 3.40 & 3.57 & 0.17 & 3.67 & 0.27 \\
\hline $\mathrm{N}(=\mathrm{Nc}(\mathrm{cccc} 1) \mathrm{c} 1) \mathrm{c}(\operatorname{ccc}(\mathrm{N}) \mathrm{c} 2) \mathrm{c} 2$ & 3.41 & 3.19 & -0.22 & 4.02 & 0.61 \\
\hline $\mathrm{n}(\mathrm{c}(\mathrm{ccc} 1) \mathrm{C}(\mathrm{Cl})(\mathrm{Cl}) \mathrm{Cl}) \mathrm{c} 1 \mathrm{Cl}$ & 3.41 & 3.35 & -0.06 & 3.87 & 0.46 \\
\hline $\begin{array}{l}\mathrm{N} 1(\mathrm{CCCC}) \mathrm{C}(\mathrm{C}(=\mathrm{O}) \mathrm{Nc} 2 \mathrm{c}(\mathrm{C}) \operatorname{cccc} 2 \mathrm{C}) \mathrm{C} \\
\mathrm{CCC} 1\end{array}$ & 3.41 & 3.44 & 0.03 & 3.31 & -0.10 \\
\hline $\mathrm{BrC}(\mathrm{Br})(\mathrm{Br}) \mathrm{Br}$ & 3.42 & 2.80 & -0.62 & 3.30 & -0.12 \\
\hline $\mathrm{C}(\mathrm{C}(\mathrm{C}) \mathrm{C})(\mathrm{C}) \mathrm{C}$ & 3.42 & 3.14 & -0.28 & 2.84 & -0.58 \\
\hline $\mathrm{c}(\mathrm{c}(\mathrm{ccc} 1) \mathrm{Cl})(\mathrm{c} 1) \mathrm{C}$ & 3.42 & 3.18 & -0.24 & 3.27 & -0.15 \\
\hline $\mathrm{c}(\mathrm{ccc}(\mathrm{c} 1) \mathrm{Br})(\mathrm{c} 1) \mathrm{C}$ & 3.42 & 3.43 & 0.01 & 3.35 & -0.07 \\
\hline $\mathrm{O}=\mathrm{C}(\mathrm{O}) \mathrm{Ccccccc}$ & 3.42 & 3.52 & 0.10 & 3.47 & 0.05 \\
\hline$c(c c(c c 1 C) C)(c 1) C$ & 3.42 & 3.63 & 0.21 & 3.64 & 0.22 \\
\hline$c(c(c c c 1) \mathrm{Cl})(\mathrm{c} 1) \mathrm{Cl}$ & 3.43 & 3.28 & -0.15 & 3.45 & 0.02 \\
\hline $\mathrm{C}(\mathrm{CCCC} 1) \mathrm{C} 1$ & 3.44 & 3.18 & -0.26 & 3.46 & 0.02 \\
\hline$c(c c c(c 1) \mathrm{Cl})(\mathrm{c} 1) \mathrm{Cl}$ & 3.44 & 3.28 & -0.16 & 3.46 & 0.02 \\
\hline $\begin{array}{l}\mathrm{c} 12 \mathrm{nccc}(\mathrm{C}(\mathrm{O}) \mathrm{C} 3 \mathrm{~N} 4 \mathrm{CCC}(\mathrm{C}(\mathrm{C}=\mathrm{C}) \mathrm{C} 4) \\
\mathrm{C} 3) \mathrm{c} 1 \mathrm{cc}(\mathrm{OC}) \mathrm{cc} 2\end{array}$ & 3.44 & 3.29 & -0.15 & 2.82 & -0.62 \\
\hline Oc(c(ccc10)CCCCCC)c1 & 3.45 & 4.04 & 0.59 & 3.77 & 0.32 \\
\hline $\mathrm{N}(\mathrm{c}(\mathrm{c}(\mathrm{c}(\mathrm{ccc} 1) \mathrm{cc} 2) \mathrm{c} 1) \mathrm{c} 2)(\mathrm{C}) \mathrm{C}$ & 3.46 & 3.35 & -0.11 & 3.67 & 0.21 \\
\hline $\mathrm{O}=\mathrm{C}(\mathrm{OOC}(=\mathrm{O}) \mathrm{c}(\operatorname{cccc} 1) \mathrm{c} 1) \mathrm{c}(\operatorname{ccc} 2) \mathrm{c} 2$ & 3.46 & 3.43 & -0.03 & 2.75 & -0.71 \\
\hline $\mathrm{O}(\mathrm{c}(\mathrm{ccc}(\mathrm{c} 1 \mathrm{ccc} 2) \mathrm{c} 2) \mathrm{c} 1) \mathrm{C}$ & 3.47 & 3.25 & -0.22 & 3.37 & -0.10 \\
\hline$O C C=C(C C C=C(C) C) C$ & 3.47 & 3.47 & 0.00 & 2.89 & -0.58 \\
\hline $\mathrm{N}(=\mathrm{C}=\mathrm{S}) \mathrm{CC}(\operatorname{cccc} 1) \mathrm{c} 1$ & 3.47 & 3.50 & 0.03 & 3.41 & -0.06 \\
\hline $\mathrm{c}(\mathrm{C}(=\mathrm{C}) \mathrm{C})(\operatorname{cccc} 1) \mathrm{c} 1$ & 3.48 & 3.44 & -0.04 & 3.31 & -0.17 \\
\hline $\mathrm{Oc}(\mathrm{c}(\operatorname{ccc} 1 \mathrm{C}(\mathrm{C}) \mathrm{C}) \mathrm{C}) \mathrm{c} 1$ & 3.49 & 3.52 & 0.03 & 3.20 & -0.29 \\
\hline$c(c(c c c 1) C c C 2)(c 1) C 2$ & 3.49 & 3.96 & 0.47 & 3.79 & 0.30 \\
\hline $\begin{array}{l}\mathrm{O}=\mathrm{C} 1 \mathrm{C}=\mathrm{C} 2 \mathrm{CCC} 3 \mathrm{C} 4 \mathrm{CC}(\mathrm{C}) \mathrm{C}(\mathrm{OC}(=\mathrm{O}) \\
\mathrm{CC}) \\
(\mathrm{C}(=\mathrm{O}) \mathrm{CCl}) \mathrm{C} 4(\mathrm{C}) \mathrm{CC}(\mathrm{O}) \mathrm{C} 3(\mathrm{~F}) \mathrm{C} 2(\mathrm{C}) \mathrm{C} \\
=\mathrm{C} 1\end{array}$ & 3.50 & 2.98 & -0.52 & 3.49 & -0.01 \\
\hline $\begin{array}{l}\mathrm{O}=\mathrm{C}(\mathrm{c}(\mathrm{c}(\mathrm{C}(=\mathrm{O}) \mathrm{c} 1 \mathrm{c}(\mathrm{N}) \mathrm{cc}(\mathrm{OC}) \mathrm{c} 2 \mathrm{~N}) \mathrm{ccc} \\
3) \mathrm{c} 3) \mathrm{c} 12\end{array}$ & 3.50 & 3.24 & -0.26 & 2.82 & -0.68 \\
\hline $\mathrm{N}(\mathrm{c}(\operatorname{cccc} 1) \mathrm{c} 1) \mathrm{c}(\operatorname{cccc} 2) \mathrm{c} 2$ & 3.50 & 3.29 & -0.21 & 3.34 & -0.16 \\
\hline $\begin{array}{l}\mathrm{O}=\mathrm{C}(\mathrm{O}) \mathrm{CCC}(\mathrm{C}(\mathrm{C}(\mathrm{C}(\mathrm{C}(\mathrm{C}(\mathrm{C}(\mathrm{C}(\mathrm{C} 1) \mathrm{CC} \\
(\mathrm{O}) \mathrm{C} 2)(\mathrm{C} 2) \mathrm{C}) \mathrm{C} 3) \mathrm{C} 1) \mathrm{C} 4) \\
(\mathrm{C} 3 \mathrm{O}) \mathrm{C}) \mathrm{C} 4) \mathrm{C}\end{array}$ & 3.50 & 5.06 & 1.56 & 3.30 & -0.20 \\
\hline $\mathrm{n} 1 \mathrm{cc}(\mathrm{C}(=\mathrm{O}) \mathrm{OCCCCCC}) \mathrm{ccc} 1$ & 3.51 & 3.10 & -0.41 & 3.06 & -0.45 \\
\hline $\mathrm{O}=\mathrm{C}(\mathrm{C}(\mathrm{CC} 1 \mathrm{C} 2)(\mathrm{C} 2) \mathrm{C}) \mathrm{C} 1(\mathrm{C}) \mathrm{C}$ & 3.52 & 3.04 & -0.48 & 2.54 & -0.98 \\
\hline $\begin{array}{l}\operatorname{c1ccc} 2 \mathrm{Sc} 3 \operatorname{ccc}(\mathrm{C}(\# \mathrm{~N})) \operatorname{cc} 3 \mathrm{~N}(\mathrm{CCCN} 4 \mathrm{C} \\
\mathrm{cC}(\mathrm{O}) \mathrm{Cc} 4) \mathrm{c} 2 \mathrm{c} 1\end{array}$ & 3.52 & 3.93 & 0.41 & 3.78 & 0.26 \\
\hline $\mathrm{c}(\operatorname{cccc} 1 \mathrm{Cl})(\mathrm{c} 1) \mathrm{Cl}$ & 3.53 & 3.28 & -0.25 & 3.45 & -0.08 \\
\hline$c(c(c c c 1) C)(c 1) C C$ & 3.53 & 3.58 & 0.05 & 3.87 & 0.34 \\
\hline $\begin{array}{l}\operatorname{COP}(=\mathrm{O}) \\
(\mathrm{OC}) \mathrm{OC}(=\mathrm{CCl}) \mathrm{c} 1 \mathrm{cc}(\mathrm{Cl}) \mathrm{c}(\mathrm{Cl}) \mathrm{cc} 1 \mathrm{Cl}\end{array}$ & 3.53 & 3.81 & 0.28 & 3.93 & 0.40 \\
\hline $\begin{array}{l}\mathrm{N}(=\mathrm{O})(=\mathrm{O}) \mathrm{c}(\mathrm{cc}(\mathrm{N}(=\mathrm{O}) \\
(=\mathrm{O})) \mathrm{c}(\mathrm{O}) \mathrm{c} 1 \mathrm{C}(\mathrm{CC}) \mathrm{C}) \mathrm{c} 1\end{array}$ & 3.56 & 3.67 & 0.11 & 3.37 & -0.19 \\
\hline $\mathrm{O}=\mathrm{C}(\mathrm{OCc}(\operatorname{cccc} 1) \mathrm{c} 1) \mathrm{c}(\operatorname{ccc}(\mathrm{O}) \mathrm{c} 2) \mathrm{c} 2$ & 3.56 & 3.70 & 0.14 & 3.66 & 0.10 \\
\hline $\mathrm{n} 1 \mathrm{cc}(\mathrm{C}(=\mathrm{O}) \mathrm{OCCC}) \operatorname{ccc} 1 \mathrm{C}(=\mathrm{O}) \mathrm{OCCC}$ & 3.57 & 2.12 & -1.45 & 2.61 & -0.96 \\
\hline $\mathrm{O}=\mathrm{C}(\mathrm{OCCCC}) \mathrm{c}(\operatorname{ccc}(\mathrm{O}) \mathrm{c} 1) \mathrm{c} 1$ & 3.57 & 3.47 & -0.10 & 3.81 & 0.24 \\
\hline Oc1c $(\mathrm{Cl}) \operatorname{cc}(\mathrm{Cl}) \mathrm{c}(\mathrm{Cl}) \mathrm{c} 10$ & 3.60 & 2.97 & -0.63 & 3.55 & -0.05 \\
\hline $\mathrm{C}(\mathrm{CC})(\mathrm{CC}) \mathrm{C}$ & 3.60 & 3.21 & -0.39 & 3.98 & 0.38 \\
\hline $\begin{array}{l}\mathrm{C} 1=\mathrm{CC}(=\mathrm{O}) \mathrm{C}=\mathrm{C} 2 \mathrm{CCC} 3 \mathrm{C} 4 \mathrm{CC}(\mathrm{C}) \mathrm{C}(\mathrm{O} \\
\mathrm{C}(=\mathrm{O}) \mathrm{CCCC}) \\
(\mathrm{C}=\mathrm{O}) \mathrm{CO}) \mathrm{C} 4(\mathrm{C}) \mathrm{CC}(\mathrm{O}) \mathrm{C} 3(\mathrm{~F}) \mathrm{C} 12 \mathrm{C}\end{array}$ & 3.60 & 3.94 & 0.34 & 3.76 & 0.16 \\
\hline $\begin{array}{l}(\mathrm{C}(=\mathrm{O}) \mathrm{CO}) \mathrm{C} 4(\mathrm{C}) \mathrm{CC}(\mathrm{O}) \mathrm{C} 3(\mathrm{~F}) \mathrm{C} 12 \mathrm{C} \\
\mathrm{C}(\mathrm{CCCC} 1)(\mathrm{C} 1) \mathrm{C}\end{array}$ & 3.61 & 3.59 & -0.02 & 3.90 & 0.29 \\
\hline Cc1ccc(cc1)CC & 3.63 & 3.58 & -0.05 & 3.83 & 0.20 \\
\hline$c(\operatorname{ccc}(\mathrm{c} 1 \mathrm{C}) \mathrm{C})(\mathrm{c} 1) \mathrm{C}$ & 3.63 & 3.63 & 0.00 & 3.62 & -0.01 \\
\hline OccscccccccC & 3.64 & 3.38 & -0.26 & 3.88 & 0.24 \\
\hline $\mathrm{c}(\mathrm{c}(\mathrm{ccc} 1) \mathrm{Br})(\mathrm{c} 1) \mathrm{Br}$ & 3.64 & 3.77 & 0.13 & 3.77 & 0.13 \\
\hline $\mathrm{Oc}(\operatorname{ccc}(\mathrm{c} 1) \operatorname{CcC}) \mathrm{c} 1$ & 3.65 & 3.53 & -0.12 & 3.55 & -0.10 \\
\hline $\begin{array}{l}\mathrm{O}=\mathrm{P}(\mathrm{OC}(\mathrm{CCl}) \mathrm{CCl}) \\
(\mathrm{OC}(\mathrm{CCl}) \mathrm{CCl}) \mathrm{OC}(\mathrm{CCl}) \mathrm{CCl}\end{array}$ & 3.65 & 3.65 & 0.00 & 3.26 & -0.39 \\
\hline $\mathrm{c}(\operatorname{cccc} 1)(\mathrm{c} 1) \mathrm{C}(\mathrm{C}) \mathrm{C}$ & 3.66 & 3.45 & -0.21 & 3.67 & 0.01 \\
\hline$c(c(c(c c 1) C) C)(c 1) C$ & 3.66 & 3.63 & -0.03 & 3.63 & -0.03 \\
\hline $\mathrm{O}=\mathrm{C}(\mathrm{c}(\mathrm{c}(\mathrm{ccc} 1) \mathrm{C} c 2 \mathrm{ccc} 3) \mathrm{c} 1) \mathrm{c} 23$ & 3.66 & 3.81 & 0.15 & 3.57 & -0.09 \\
\hline $\mathrm{OC}(\mathrm{c}(\mathrm{cccc} 1) \mathrm{c} 1)$ & 3.68 & 4.38 & 0.70 & 4.31 & 0.63 \\
\hline
\end{tabular}


benzene, propyl-

methanesulfenamide, 1,1-dichloro-N-

[(dimethylamino)sulfonyl]-1-fluoro-N-phenyl-

4,7-methano-1H-isoindole-1,3(2H)-dione, 2-(2-

ethylhexyl)-3a,4,7,7a-tetrahydro-

benzoic acid, 5-[2-chloro-4-

(trifluoromethyl)phenoxy]-2-nitro-

1,2-benzenediol, 3,4,5-trichloro-

cyclohexanamine, $\mathrm{N}$-cyclohexyl-N-methyl-

$3 \mathrm{H}$-indol-3-one, 2-(1,3-dihydro-3-oxo-2H-indol-2-

ylidene)-1,2-dihydro-

$9 \mathrm{H}$-carbazole

phenol, 2,3,4-trichloro-6-methoxy-

cyclohexane, 1,2,3,4,5,6-hexachloro-,

$(1 \mathrm{a}, 2 \mathrm{a}, 3 \mathrm{~b}, 4 \mathrm{a}, 5 \mathrm{a}, 6 \mathrm{~b})$ -

phenol, 3,4,5-trichloro-2,6-dimethoxy-

9,10-anthracenedione, 1-amino-

benzenamine, 4-nitro-N-phenyl-

propanedioic acid, 1,3-dithiol-2-ylidene-, bis(1-

methylethyl) ester

ethanol, 2-butoxy-, phosphate (3:1)

\section{2,2-bithiophene \\ pregna-1,4-diene-3,11,20-trione, 21-chloro-9-fluoro- 16-methyl-17-(1-oxobutoxy)-, (16-beta)-}

phenol, 3,4,5-trichloro-2-methoxy-

1-nonanol

ethanamine, N,N-dimethyl-2-[(2-

methylphenyl)phenylmethoxy]-

methanone, (2-hydroxy-4-methoxyphenyl)phenyl-

phenol, 2,6-bis(1-methylethyl)-

benzene, 1,4-dibromo-

benzoic acid, 2-hydroxy-, propyl ester

hexane, 1-bromo-

phosphorothioic acid, o,o-diethyl o-[6-methyl-2-(1-

methylethyl)-4-pyrimidinyl] ester

butane, 2,2-dimethyl-

diazene, diphenyl-

6-octenal, 3,7-dimethyl-

benzoic acid, butyl ester

3-buten-2-one, 4-(2,6,6-trimethyl-1-cyclohexen-1yl)-

benzoic acid, 4-(1,1-dimethylethyl)-

3-buten-2-one, 4-(2,6,6-trimethyl-2-cyclohexen-1yl)-, (E)-

pregna-1,4-diene-3,20-dione, 21-(2,2-dimethyl-1oxopropoxy)-6,9-difluoro-11,17-dihydroxy-16-

methyl-, (6-alpha,11-beta,16-alpha)-

naphthalene, 2-methyl-

10-undecenoic acid

methanone, bis[4-(dimethylamino)phenyl]-

naphthalene, 1-methyl-

thioperoxydicarbonic diamide, tetraethyl-

methanesulfenamide, 1,1-dichloro- $\mathrm{N}$ -

[(dimethylamino)sulfonyl]-1-fluoro-N-(4-

methylphenyl)-

hexane

naphthalene, 1-chloro-

benzenamine, 4,4'-methylenebis[2-chloro-]

6-octen-1-ol, 3,7-dimethyl-

acenaphthylene, 1,2-dihydro- $(\mathrm{c}(\operatorname{ccc} 2) \mathrm{c} 2) \mathrm{c}(\operatorname{cccc} 3) \mathrm{c} 3$

$\mathrm{c}(\mathrm{cccc} 1)(\mathrm{c} 1) \mathrm{CCC}$

$\mathrm{CN}(\mathrm{C}) \mathrm{S}(=\mathrm{O})(=\mathrm{O}) \mathrm{N}(\mathrm{SC}(\mathrm{F})$

3.52

$(\mathrm{Cl}) \mathrm{Cl}) \mathrm{c} 1 \mathrm{ccccc} 1$

$\operatorname{CCCCC}(\mathrm{CC}) \mathrm{CN} 3 \mathrm{C}(=\mathrm{O}) \mathrm{C} 2 \mathrm{C} 1 \mathrm{CC}(\mathrm{C}=$

$\mathrm{C} 1) \mathrm{C} 2 \mathrm{C} 3(=\mathrm{O})$

$\mathrm{O}=\mathrm{C}(\mathrm{O}) \mathrm{c}(\mathrm{c}(\mathrm{N}(=\mathrm{O})$

$(=\mathrm{O})) \operatorname{ccc} 1 \mathrm{Oc}(\mathrm{c}(\mathrm{cc}(\mathrm{c} 2) \mathrm{C}(\mathrm{F})$

(F)F)Cl)c2)c1

$\mathrm{c} 1(\mathrm{O}) \mathrm{c}(\mathrm{O}) \mathrm{c}(\mathrm{Cl}) \mathrm{c}(\mathrm{Cl}) \mathrm{c}(\mathrm{Cl}) \mathrm{c} 1$

$\mathrm{N}(\mathrm{C}(\mathrm{CCCC} 1) \mathrm{C} 1)(\mathrm{C}(\mathrm{CCCC} 2) \mathrm{C} 2) \mathrm{C} \quad 3.71$

$\mathrm{O}=\mathrm{C}(\mathrm{c}(\mathrm{c}(\mathrm{N} 1) \mathrm{ccc} 2) \mathrm{c} 2) \mathrm{C} 1=\mathrm{C}(\mathrm{Nc}(\mathrm{c} 3 \mathrm{ccc}$

4)c4)C3(=O)

$\mathrm{n}(\mathrm{c}(\mathrm{c}(\mathrm{c} 1 \mathrm{cccc} 2) \mathrm{ccc} 3) \mathrm{c} 3) \mathrm{c} 12$

$\mathrm{COc} 1 \mathrm{cc}(\mathrm{Cl}) \mathrm{c}(\mathrm{Cl}) \mathrm{c}(\mathrm{Cl}) \mathrm{c} 1 \mathrm{O}$

$\mathrm{C}(\mathrm{C}(\mathrm{C}(\mathrm{C}(\mathrm{C} 1 \mathrm{Cl}) \mathrm{Cl}) \mathrm{Cl}) \mathrm{Cl})(\mathrm{C} 1 \mathrm{Cl}) \mathrm{Cl}$

3.70

2.72

$-0.17$

3.86

0.17

$\mathrm{Oc} 1 \mathrm{c}(\mathrm{OC}) \mathrm{c}(\mathrm{Cl}) \mathrm{c}(\mathrm{Cl}) \mathrm{c}(\mathrm{Cl}) \mathrm{c} 1 \mathrm{OC}$

3.76

0.06

3.78

$-0.15$

$\mathrm{O}=\mathrm{C}(\mathrm{c}(\mathrm{c}(\mathrm{c}(\mathrm{N}) \mathrm{cc} 1) \mathrm{C}(=\mathrm{O}) \mathrm{c} 2 \mathrm{cccc} 3) \mathrm{c} 1) \mathrm{c}$ 23

$\mathrm{N}(=\mathrm{O})(=\mathrm{O}) \mathrm{c}(\operatorname{ccc}(\mathrm{Nc}(\operatorname{cccc} 1) \mathrm{c} 1) \mathrm{c} 2) \mathrm{c} 2$

3.70

4.17

0.47

3.87

0.08

$\mathrm{S} 1 \mathrm{C}=\mathrm{CSC} 1=\mathrm{C}(\mathrm{C}(=\mathrm{O}) \mathrm{OC}(\mathrm{C}) \mathrm{C}) \mathrm{C}(=\mathrm{O})$

$\mathrm{OC}(\mathrm{C}) \mathrm{C}$

$\mathrm{O}=\mathrm{P}(\mathrm{OCCOCCCC})$

(OCCOCCCC)OCCOCCCC

$\mathrm{S} 1 \mathrm{C}=\mathrm{CC}=\mathrm{C} 1 \mathrm{C} 2=\mathrm{CC}=\mathrm{CS} 2$

$\mathrm{O}=\mathrm{C} 1 \mathrm{C}=\mathrm{C} 2 \mathrm{CCC} 3 \mathrm{C} 4 \mathrm{CC}(\mathrm{C}) \mathrm{C}(\mathrm{OC}(=\mathrm{O})$

$\mathrm{CCC})$

$(\mathrm{C}(=\mathrm{O}) \mathrm{CCl}) \mathrm{C} 4(\mathrm{C}) \mathrm{CC}(=\mathrm{O}) \mathrm{C} 3(\mathrm{~F}) \mathrm{C} 2(\mathrm{C})$

$\mathrm{C}=\mathrm{C} 1$

$\mathrm{COc} 1 \mathrm{c}(\mathrm{O}) \mathrm{cc}(\mathrm{Cl}) \mathrm{c}(\mathrm{Cl}) \mathrm{c} 1 \mathrm{Cl}$

OCCCCCCCCC

2.97

4.58

$-0.74$

-0.74
0.87

3.53
4.85

$-0.61 \quad 2.76$

3.23

$-0.49$

2.76

3.69

$-0.45$

3.81

3.94

0.09

4.26

0.54

$\begin{array}{lll}3.10 & -0.64 & 3.81 \\ 3.53 & -0.21 & 3.21\end{array}$

0.07

3.74

3.69

$-0.05$

3.62

$-0.53$

$-0.12$

2.57

$-1.18$

3.58

$-0.17$

3.00

$-0.75$

3.31

$-0.44$

3.75

3.40

$-0.35$

4.06

0.31

$\begin{array}{lll}3.67 & -0.09 & 3.77\end{array}$

0.01

$\mathrm{O}(\mathrm{C}(\mathrm{c}(\mathrm{cccc} 1) \mathrm{c} 1) \mathrm{c}(\mathrm{c}(\mathrm{ccc} 2) \mathrm{C}) \mathrm{c} 2) \mathrm{CCN}($

$\mathrm{C}) \mathrm{C}$

$\mathrm{O}=\mathrm{C}(\mathrm{c}(\mathrm{cccc} 1) \mathrm{c} 1) \mathrm{c}(\mathrm{c}(\mathrm{O}) \mathrm{cc}(\mathrm{OC}) \mathrm{c} 2) \mathrm{c} 2$

$\mathrm{Oc}(\mathrm{c}(\operatorname{ccc} 1) \mathrm{C}(\mathrm{C}) \mathrm{C}) \mathrm{c} 1 \mathrm{C}(\mathrm{C}) \mathrm{C}$

$\mathrm{c}(\mathrm{ccc}(\mathrm{c} 1) \mathrm{Br})(\mathrm{c} 1) \mathrm{Br}$

$\mathrm{O}=\mathrm{C}(\mathrm{OCCC}) \mathrm{c}(\mathrm{c}(\mathrm{O}) \mathrm{ccc} 1) \mathrm{c} 1$

$\mathrm{BrCCCCCC}$

$\mathrm{O}(\mathrm{P}(\mathrm{OCC})$

$(\mathrm{Oc}(\mathrm{nc}(\mathrm{nc} 1 \mathrm{C}) \mathrm{C}(\mathrm{C}) \mathrm{C}) \mathrm{c} 1)=\mathrm{S}) \mathrm{CC}$

$\mathrm{C}(\mathrm{CC})(\mathrm{C})(\mathrm{C}) \mathrm{C}$

$\mathrm{N}(=\mathrm{Nc}(\operatorname{cccc} 1) \mathrm{c} 1) \mathrm{c}(\operatorname{cccc} 2) \mathrm{c} 2$

$\mathrm{O}=\mathrm{CCC}(\mathrm{CCC}=\mathrm{C}(\mathrm{C}) \mathrm{C}) \mathrm{C}$

$\mathrm{O}=\mathrm{C}(\mathrm{OCCCC}) \mathrm{c}(\operatorname{cccc} 1) \mathrm{c} 1$

$\mathrm{O}=\mathrm{C}(\mathrm{C}=\mathrm{CC}(=\mathrm{C}(\mathrm{CCC} 1) \mathrm{C}) \mathrm{C} 1(\mathrm{C}) \mathrm{C}) \mathrm{C}$

3.77

3.27

$-0.50$

3.81

0.04

$\begin{array}{lllll}3.77 & 3.30 & -0.47 & 3.76 & -0.01\end{array}$

$\begin{array}{lllll}3.77 & 3.65 & -0.12 & 3.50 & -0.27\end{array}$

$\mathrm{O}=\mathrm{C}(\mathrm{O}) \mathrm{c}(\operatorname{ccc}(\mathrm{c} 1) \mathrm{C}(\mathrm{C})(\mathrm{C}) \mathrm{C}) \mathrm{c} 1$

$\mathrm{O}=\mathrm{C}(\mathrm{C}=\mathrm{CC}(\mathrm{C}(=\mathrm{CCC} 1) \mathrm{C}) \mathrm{C} 1(\mathrm{C}) \mathrm{C}) \mathrm{C}$

3.79

3.79

3.52

$-0.27$

3.50

3.57

$-0.22$

3.35

$-0.44$

3.77

$-0.02$

3.81

0.02

3.80

3.80

3.59

$-0.21$

$-0.08$

$-0.61$

0.08

3.86

0.05

3.88

0.64

3.82

3.18

$-0.64$

4.45

$-0.08$

4.11

0.29

3.74

3.53

$-0.30$

4.30

0.48

$-0.58$

$\begin{array}{lllll}3.84 & 3.30 & -0.54 & 3.40 & -0.44\end{array}$

$\begin{array}{lllll}3.84 & 4.42 & 0.58 & 4.11 & 0.27\end{array}$

3.85

3.78

$-0.07$

3.32

$-0.53$

$\mathrm{O}=\mathrm{C} 1 \mathrm{C}=\mathrm{C} 2 \mathrm{C}(\mathrm{F}) \mathrm{CC} 3 \mathrm{C} 4 \mathrm{CC}(\mathrm{C}) \mathrm{C}(\mathrm{O})$

4.29

0.44

4.14

0.29

3.86

3.69

$-0.17$

3.21

$-0.65$

$\mathrm{C}(=\mathrm{O}) \mathrm{COC}(=\mathrm{O}) \mathrm{C}(\mathrm{C})$

(C)C $\mathrm{C} 4(\mathrm{C}) \mathrm{CC}(\mathrm{O}) \mathrm{C} 3(\mathrm{~F}) \mathrm{C} 2(\mathrm{C}) \mathrm{C}=\mathrm{C} 1$

$\mathrm{c}(\mathrm{c}(\mathrm{ccc} 1 \mathrm{C}) \mathrm{ccc} 2)(\mathrm{c} 2) \mathrm{c} 1$

3.72

$-0.14$

3.83

$\mathrm{O}=\mathrm{C}(\mathrm{O}) \operatorname{CCCCCCCCC}=\mathrm{C}$

3.86
3.86

$\mathrm{O}=\mathrm{C}(\mathrm{c}(\operatorname{ccc}(\mathrm{N}(\mathrm{C}) \mathrm{C}) \mathrm{c} 1) \mathrm{c} 1) \mathrm{c}(\operatorname{ccc}(\mathrm{N}(\mathrm{C}) \mathrm{C}$ ) $\mathrm{c} 2) \mathrm{c} 2$

$\mathrm{c}(\mathrm{c}(\mathrm{c}(\mathrm{cc} 1) \mathrm{C}) \mathrm{ccc} 2)(\mathrm{c} 2) \mathrm{c} 1$

$\mathrm{N}(\mathrm{C}(=\mathrm{S}) \mathrm{SSC}(\mathrm{N}(\mathrm{CC}) \mathrm{CC})=\mathrm{S})(\mathrm{CC}) \mathrm{CC}$

$\mathrm{CN}(\mathrm{C}) \mathrm{S}(=\mathrm{O})(=\mathrm{O}) \mathrm{N}(\mathrm{SC}(\mathrm{F})$

4.37

0.51

3.84

$-0.03$

(Cl)Cl)c1ccc(C)cc1

3.72

$-0.15$

3.74

$-0.13$

$\mathrm{C}(\mathrm{CCCC}) \mathrm{C}$

$\mathrm{c}(\mathrm{c}(\mathrm{c}(\mathrm{cc} 1) \mathrm{Cl}) \mathrm{ccc} 2)(\mathrm{c} 2) \mathrm{c} 1$

3.84

$-0.03$

$\mathrm{Nc}(\mathrm{c}(\mathrm{cc}(\mathrm{c} 1) \mathrm{Cc}(\mathrm{ccc}(\mathrm{N}) \mathrm{c} 2 \mathrm{Cl}) \mathrm{c} 2) \mathrm{Cl}) \mathrm{c} 1$

$\operatorname{OCCC}(\mathrm{CCC}=\mathrm{C}(\mathrm{C}) \mathrm{C}) \mathrm{C}$

$\mathrm{c}(\mathrm{c}(\mathrm{ccc} 1) \mathrm{ccc} 2)(\mathrm{c} 1 \mathrm{CC} 3) \mathrm{c} 23$

$-0.63$

3.88

0.00

3.90

3.29

$-0.61$

4.02

0.12

3.90

3.81

$-0.09$

3.95

0.05

3.47

$-0.44$

3.72

$-0.19$

$\begin{array}{lllll}3.91 & 3.56 & -0.35 & 3.48 & -0.43\end{array}$

$\begin{array}{lllll}3.92 & 4.15 & 0.23 & 4.01 & 0.09\end{array}$ 
phenol, 4-chloro-5-methyl-2-(1-methylethyl)-

cyclohexene, 4-ethenyl-

1,6-octadien-3-ol, 3,7-dimethyl-, acetate

propanamide, N-[4-(methoxymethyl)-1-[2-(2-

thienyl)ethyl]-4-piperidinyl]-N-phenyl-, 2-hydroxy-

1,2,3-propanetricarboxylate $(1: 1)$

benzene, 1,2-dichloro-4-methyl-

terpineol, acetate

benzoic acid, phenylmethyl ester

benzeneacetic acid, alpha-methyl-4-(2-

methylpropyl)-

benzene, 1,4-dichloro-2-methyl-

1,1-biphenyl

naphthalene, 2-chloro-

2,6-octadien-1-ol, 3,7-dimethyl-, acetate

1-heptene

phosphoric acid tributyl ester

propanenitrile, 3-[[4-[(2-chloro-4-

nitrophenyl)azo]phenyl](2-hydroxyethyl)amino]-

benzene, 1,2,4,5-tetramethyl-

benzene, 1,2,3,4-tetramethyl-

cyclohexanol, 5-methyl-2-(1-methylethyl)-, acetate

benzene, 1,2,3-trichloro-4,5-dimethoxy-

estra-1,3,5(10)-triene-3,17-diol (17-beta)-

benzoic acid, 2-phenylethyl ester

benzene, 1,2,4-trichloro-

benzene, 1,2,3-trichloro-

pregna-1,4-diene-3,20-dione, 9-fluoro-11-hydroxy-

16-methyl-17,21-bis(1-oxopropoxy)-, (11-beta,16-

beta)-

propanenitrile, 3-[[2-(acetyloxy)ethyl][4-[(6-nitro-2benzothiazolyl)azo]phenyl]amino]-

\section{2-undecanone}

decanoic acid

benzene, methyl(1-methylethyl)-

9,10-anthracenedione, 1-(methylamino)-

benzene, 1,2,3,5-tetramethyl-

[1]benzopyrano[3,4-b]furo[2,3-H][1] benzopyran-

6(6aH)-one, 1,2,12,12a-tetrahydro-8,9-dimethoxy-2(1-methylethenyl)-

benzene, (1,1-dimethylethyl)-

1,2-benzenedicarboxylic acid, bis(2-methylpropyl)

ester

dibenzofuran

phenol, 2,4,6-tribromo-

benzene, 1,1-methylenebis-

ethane, hexachloro-

1-butanol, 3-methyl-, benzoate

$10 \mathrm{H}$-phenothiazine

cholan-24-oic acid, 3,7-dihydroxy-, (3-alpha,5 ,7alpha)-

(-)-flurbiprofen

bicyclo[3.1.1]heptane, 6,6-dimethyl-2-methylene-

1,3,7-octatriene, 3,7-dimethyl-

1,6-octadiene, 7-methyl-3-methylene-

$9 \mathrm{H}$-fluorene

benzene, ethenylethyl-

benzene, 1,3,5-trichloro-

1-piperazineethanol, 4-[3-(2-chloro-10H-

phenothiazin-10yl)propyl]-

1-naphthalenamine, N-phenyl-

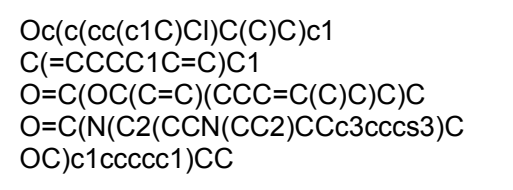

$\begin{array}{llccc}3.92 & 4.16 & 0.24 & 3.75 & -0.17 \\ 3.93 & 3.73 & -0.20 & 3.49 & -0.44 \\ 3.93 & 4.39 & 0.46 & 4.03 & 0.10\end{array}$

$\mathrm{c}(\mathrm{ccc}(\mathrm{c} 1 \mathrm{Cl}) \mathrm{Cl})(\mathrm{c} 1) \mathrm{C}$

$3.95 \quad 3.83$

$\mathrm{O}=\mathrm{C}(\mathrm{OC}(\mathrm{C} 1 \mathrm{CC}=\mathrm{C}(\mathrm{CC} 1) \mathrm{C})(\mathrm{C}) \mathrm{C}) \mathrm{C}$

$\mathrm{O}=\mathrm{C}(\mathrm{OCc}(\mathrm{cccc} 1) \mathrm{c} 1) \mathrm{c}(\mathrm{cccc} 2) \mathrm{c} 2$

$\mathrm{O}=\mathrm{C}(\mathrm{O}) \mathrm{C}(\mathrm{c}(\operatorname{ccc}(\mathrm{c} 1) \mathrm{CC}(\mathrm{C}) \mathrm{C}) \mathrm{c} 1) \mathrm{C}$

$3.97 \quad 3.54$

$3.97 \quad 3.79$

$\mathrm{c}(\operatorname{ccc}(\mathrm{c} 1 \mathrm{C}) \mathrm{Cl})(\mathrm{c} 1) \mathrm{Cl}$

$\mathrm{c}(\mathrm{c}(\mathrm{cccc} 1) \mathrm{c} 1)(\mathrm{cccc} 2) \mathrm{c} 2$

$\mathrm{c}(\mathrm{c}(\mathrm{ccc} 1 \mathrm{Cl}) \mathrm{ccc} 2)(\mathrm{c} 2) \mathrm{c} 1$

$3.97 \quad 3.83$

$3.98 \quad 3.76$

$3.98 \quad 3.81$

$\mathrm{O}=\mathrm{C}(\mathrm{OCC}=\mathrm{C}(\mathrm{CCC}=\mathrm{C}(\mathrm{C}) \mathrm{C}) \mathrm{C}) \mathrm{C}$

$\mathrm{C}(=\mathrm{C}) \mathrm{CCCCC}$

$\mathrm{O}=\mathrm{P}(\mathrm{OCCCC})(\mathrm{OCCCC}) \mathrm{OCCCC}$

$\mathrm{N}(=\mathrm{O})$

$(=\mathrm{O}) \mathrm{c}(\operatorname{ccc}(\mathrm{N}=\mathrm{Nc}(\operatorname{ccc}(\mathrm{N}(\mathrm{CCC}(\# \mathrm{~N})) \mathrm{CC}$

O)c1)c1)c2Cl)c2

$\mathrm{c}(\mathrm{c}(\mathrm{cc}(\mathrm{c} 1 \mathrm{C}) \mathrm{C}) \mathrm{C})(\mathrm{c} 1) \mathrm{C}$

$\mathrm{c}(\mathrm{c}(\mathrm{c}(\mathrm{c}(\mathrm{c} 1) \mathrm{C}) \mathrm{C}) \mathrm{C})(\mathrm{c} 1) \mathrm{C}$

$\operatorname{CC} 1 \mathrm{CCC}(\mathrm{C}(\mathrm{C}) \mathrm{C}) \mathrm{C}(\mathrm{C} 1) \mathrm{OC}(=\mathrm{O})(\mathrm{C})$

$\mathrm{COc} 1 \mathrm{c}(\mathrm{OC}) \mathrm{c}(\mathrm{Cl}) \mathrm{c}(\mathrm{Cl}) \mathrm{c}(\mathrm{Cl}) \mathrm{c} 1$

$\mathrm{CC} 34 \mathrm{CCC} 1 \mathrm{C}(\mathrm{CCc} 2 \mathrm{cc}(\mathrm{O}) \operatorname{ccc} 12) \mathrm{C} 3 \mathrm{C}$ $\mathrm{CC} 4 \mathrm{O}$

$\mathrm{O}=\mathrm{C}(\mathrm{OCCc}(\operatorname{cccc} 1) \mathrm{c} 1) \mathrm{c}(\operatorname{cccc} 2) \mathrm{c} 2$

$\mathrm{c}(\mathrm{ccc}(\mathrm{c} 1 \mathrm{Cl}) \mathrm{Cl})(\mathrm{c} 1) \mathrm{Cl}$

$\mathrm{c}(\mathrm{c}(\mathrm{c}(\mathrm{cc} 1) \mathrm{Cl}) \mathrm{Cl})(\mathrm{c} 1) \mathrm{Cl}$

$\mathrm{C} 1=\mathrm{CC}(=\mathrm{O}) \mathrm{C}=\mathrm{C} 2 \mathrm{CCC} 3 \mathrm{C} 4 \mathrm{CC}(\mathrm{C}) \mathrm{C}(\mathrm{O}$

3.98

3.99

4.00

4.00

4.48

3.64

3.82

3.87

$-0.33$

3.40

$-0.55$

$\mathrm{C}(=\mathrm{O}) \mathrm{CC})$

$(\mathrm{C}(=\mathrm{O}) \mathrm{COC}(=\mathrm{O}) \mathrm{CC}) \mathrm{C} 4(\mathrm{C}) \mathrm{CC}(\mathrm{O}) \mathrm{C} 3($

F)C12C

$\mathrm{N} 1 \mathrm{c} 2 \mathrm{ccc}(\mathrm{N}(=\mathrm{O})$

$(=\mathrm{O})) \operatorname{cc} 2 \mathrm{SC}=1 \mathrm{~N}=\mathrm{Nc} 3 \operatorname{ccc}(\mathrm{N}(\mathrm{CCC}(\# \mathrm{~N})$

) $\operatorname{CCOC}(=0) \mathrm{C}) \mathrm{cc} 3$

$\mathrm{O}=\mathrm{C}(\operatorname{Cccccccc}) \mathrm{C}$

$\operatorname{coc} \operatorname{cccccc}(=0) \mathrm{O}$

$\mathrm{Cc} 1 \mathrm{ccc}(\mathrm{C}(\mathrm{C}) \mathrm{C}) \mathrm{cc} 1$

$\mathrm{O}=\mathrm{C}(\mathrm{c}(\mathrm{c}(\mathrm{c}(\mathrm{NC}) \operatorname{cc} 1) \mathrm{C}(=\mathrm{O}) \mathrm{c} 2 \mathrm{cccc} 3) \mathrm{c} 1)$ c23

$\mathrm{c}(\mathrm{cc}(\mathrm{c}(\mathrm{c} 1 \mathrm{C}) \mathrm{C}) \mathrm{C})(\mathrm{c} 1) \mathrm{C}$

COc5cc4OCC $3 \mathrm{Oc} 2 \mathrm{c} 1 \mathrm{CC}(\mathrm{Oc} 1 \mathrm{ccc} 2 \mathrm{C}($

4.00

4.00

4.18

4.18

$4.00 \quad 4.39$

$4.01 \quad 3.57$

3.94

$-0.12$

3.97

0.38

$-0.43$

4.32

$\begin{array}{lll}0.18 & 3.50- & -0.54\end{array}$

$\begin{array}{lll}-0.14 & 3.97 & 0.00\end{array}$

$-0.22$

$-0.17$

0.50

$-0.35$

$-0.18$

$-0.13$

4.02

3.91

4.11

4.00

3.65

4.45

0.04

$-0.07$

0.13

0.01

$-0.35$

0.45

4.03

3.93

3.93

3.66

0.18

0.18

0.39

$-0.44$

$-0.07$

4.05

4.07

3.54

3.94

3.57

0.02

3.86

$-0.09$

$-0.12$

4.08

4.07

$-0.41$

3.38

0.05

0.07

$-0.46$

$-0.07$

$-0.44$

$-0.15$

0.06

0.02
-0.69

$=\mathrm{O}) \mathrm{C} 3 \mathrm{c} 4 \mathrm{cc} 5 \mathrm{OC}) \mathrm{C}(\mathrm{C})=\mathrm{C}$

$\mathrm{c}(\mathrm{cccc} 1)(\mathrm{c} 1) \mathrm{C}(\mathrm{C})(\mathrm{C}) \mathrm{C}$

$\mathrm{O}=\mathrm{C}(\mathrm{OCC}(\mathrm{C}) \mathrm{C}) \mathrm{c}(\mathrm{c}(\operatorname{ccc} 1) \mathrm{C}(=\mathrm{O}) \mathrm{OCC}($

C)C) 1

$\mathrm{o}(\mathrm{c}(\mathrm{c}(\mathrm{c} 1 \mathrm{cccc} 2) \mathrm{ccc} 3) \mathrm{c} 3) \mathrm{c} 12$

$\mathrm{Oc}(\mathrm{c}(\mathrm{cc}(\mathrm{c} 1) \mathrm{Br}) \mathrm{Br}) \mathrm{c} 1 \mathrm{Br}$

$\mathrm{c}(\mathrm{cccc} 1)(\mathrm{c} 1) \mathrm{Cc}(\mathrm{cccc} 2) \mathrm{c} 2$

$\mathrm{C}(\mathrm{C}(\mathrm{Cl})(\mathrm{Cl}) \mathrm{Cl})(\mathrm{Cl})(\mathrm{Cl}) \mathrm{Cl}$

$\mathrm{O}=\mathrm{C}(\mathrm{OCCC}(\mathrm{C}) \mathrm{C}) \mathrm{c}(\operatorname{cccc} 1) \mathrm{c} 1$

$\mathrm{N}(\mathrm{c}(\mathrm{c}(\mathrm{Sc} 1 \mathrm{cccc} 2) \operatorname{ccc} 3) \mathrm{c} 3) \mathrm{c} 12$

$\operatorname{CC}(\operatorname{CCC}(=0)$

(O))C3CCC4C2C(O)CC1CC $(\mathrm{O}) \mathrm{CCC}$ 1(C)C2CCC34C

$\mathrm{OC}(=\mathrm{O}) \mathrm{C}(\mathrm{C}) \mathrm{c} 1 \mathrm{cc}(\mathrm{F}) \mathrm{c}(\mathrm{c} 2 \mathrm{ccccc} 2) \mathrm{cc} 1$

$\mathrm{C}(\mathrm{C}(\mathrm{CC} 1 \mathrm{C} 2) \mathrm{C} 1(\mathrm{C}) \mathrm{C})(\mathrm{C} 2)=\mathrm{C}$

$\mathrm{C}(\mathrm{CCC}=\mathrm{C}(\mathrm{C}=\mathrm{C}) \mathrm{C})(=\mathrm{C}) \mathrm{C}$

$\mathrm{C}(\mathrm{C}=\mathrm{C})(=\mathrm{C}) \mathrm{CCC}=\mathrm{C}(\mathrm{C}) \mathrm{C}$

$\mathrm{c}(\mathrm{c}(\mathrm{c}(\mathrm{c} 1 \mathrm{ccc} 2) \mathrm{c} 2) \mathrm{ccc} 3)(\mathrm{c} 3) \mathrm{C} 1$

$\mathrm{C}=\mathrm{Cc} 1 \mathrm{ccc}(\mathrm{cc} 1) \mathrm{CC}$

$\mathrm{c}(\mathrm{cc}(\mathrm{cc} 1 \mathrm{Cl}) \mathrm{Cl})(\mathrm{c} 1) \mathrm{Cl}$

4.08

3.83

$-0.25$

4.63

0.55

3.69

$-0.40$

$-0.07$

4.25

4.09

4.10

4.02

$-0.10$

3.93

4.17

0.16
-0.16

4.07

$-0.03$

3.02

4.18

0.08

4.06

2.30

$-0.04$

$-1.80$

OCCN4CCN(CCCN2c1ccccc1Sc3cc

$\mathrm{C}(\mathrm{Cl}) \mathrm{cc} 23) \mathrm{CC} 4$

$\mathrm{N}(\mathrm{c}(\mathrm{c}(\mathrm{c}(\mathrm{ccc} 1) \mathrm{cc} 2) \mathrm{c} 1) \mathrm{c} 2) \mathrm{c}(\mathrm{cccc} 3) \mathrm{c} 3$

4.10
4.10

4.31

4.11

3.90

4.46

$-0.21$

0.35

4.49

3.98

0.38

$-0.13$

3.71

$-0.41$

3.92

$-0.20$

$4.13 \quad 4.18$

$4.14 \quad 4.02$

$4.14 \quad 4.03$

$4.15 \quad 3.72$

$4.15 \quad 3.82$

$4.15 \quad 5.06$

0.05

$-0.12$

420

4.33

3.93

$-0.43$

$-0.33$

3.59

4.19

3.01

0.07

0.19

$-0.21$

$-0.56$

0.04

$-1.14$

\section{$4.16 \quad 3.81$}

$4.16 \quad 4.35$

$4.17 \quad 4.88$

$4.17 \quad 4.88$

$4.18 \quad 4.02$

$4.19 \quad 3.93$

$4.19 \quad 3.93$

$4.20 \quad 3.82$

$-0.35$

3.57

\section{$-0.59$}

0.19

0.71

3.94

4.25

$0.71 \quad 4.32$

$-0.16$

$-0.26$

$-0.26$

4.26

3.98

4.08

$-0.38$

4.15 
diphenyl oxide

phenol, 4-cyclohexyl-

bicyclo[2.2.1] heptane, 2,2-dimethyl-3-methylene-

benzene, 2,4-dichloro-1-methyl-

1,3-cyclohexadiene, 1-methyl-4-(1-methylethyl)-

1-piperidinepropanol, alpha-bicyclo[2.2.1]hept-5en-2-yl-alpha-phenyl-

phenol, 2,2'-methylenebis[4-chloro-]

phosphoric acid, dibutyl phenyl ester

1H-indole-3-acetic acid, 1-(4-chlorobenzoyl)-5-

methoxy-2-methyl-

1,2-benzenediol, 3,4,5,6-tetrachloro-

1-propanamine, 3-dibenz[b,e]oxepin-11(6H)-

ylidene-N,N-dimethyl-

1-butanone, 4-[4-(4-chlorophenyl)-4-hydroxy-1-

piperidinyl]-1-(4-fluorophenyl)-

ethanol, 2-[ethyl[4-[(4-

nitrophenyl)azo]phenyl]amino]-

ethanol, 2,2'-[[4-[(2-chloro-4-nitrophenyl)azo]-3methylphenyl]imino]bis-

cyclopropanecarboxylic acid, 3-(3-methoxy-2methyl-3-oxo-1-propenyl)-2,2-dimethyl-, 2-methyl4-oxo-3-(2,4-pentadienyl)-2-cyclopenten-1-yl ester phenol, 2-(2H-benzotriazol-2-yl)-4-methyl-

2,2-biquinoline

piperazine, 1-acetyl-4-[4-[[2-(2,4-dichlorophenyl)-2(1H-imidazol-1-ylmethyl)-1,3-dioxolan-4-

yl]methoxy]phenyl]-

heptane, 1-bromo-

benzoic acid, 2-(phenylamino)-

9,10-anthracenedione, 2-ethyl-

2-cyclohexen-1-one, 2-[1-(ethoxyimino)butyl]-5-[2(ethylthio)propyl]-3-hydroxy-

benzene, butyl-

dibenzothiophene

2-naphthalenamine, $\mathrm{N}$-phenyl-

bicyclo[4.1.0]hept-3-ene, 3,7,7-trimethyl-

3,5-dioxa-6-aza-4-phosphaoct-6-ene-8-nitrile, 4-

ethoxy-7-phenyl-, 4-sulfide

2-propenoic acid, 2-methyl-, 2-ethyl-2-[[(2-methyl-1-

oxo-2-propenyl)oxy]methyl]-1,3-propanediyl ester

4-quinolinecarboxamide, 2-butoxy-N-[2-

(diethylamino)ethyl]-

decanoic acid, methyl ester

disulfide, diphenyl

2,5-cyclohexadiene-1,4-dione, 2,5-bis(1,1-

dimethylethyl)-

undecanoic acid

[1,1-biphenyl]-3-carboxylic acid, 2 ,4-difluoro-4hydroxy-

bicyclo[4.1.0]hept-2-ene, 3,7,7-trimethyl-

phenol, 2,3,4,6-tetrachloro-

benzene, 1,1-thiobis-

anthracene

2-propenoic acid, 3-phenyl-, 3-phenyl-2-propenyl

ester

phenanthrene

cyclohexene, 1-methyl-4-(1-methylethylidene)-

1-piperidinepropanol, alpha-cyclohexyl-alpha-

phenyl-

1,2-benzenedicarboxylic acid, dibutyl ester
$\mathrm{O}(\mathrm{c}(\mathrm{cccc} 1) \mathrm{c} 1) \mathrm{c}(\operatorname{cccc} 2) \mathrm{c} 2$

$\mathrm{Oc}(\operatorname{ccc}(\mathrm{c} 1) \mathrm{C}(\mathrm{CCCC} 2) \mathrm{C} 2) \mathrm{c} 1$

$\mathrm{C}(\mathrm{C}(\mathrm{CC} 1 \mathrm{C} 2) \mathrm{C} 2)(\mathrm{C} 1(\mathrm{C}) \mathrm{C})=\mathrm{C}$

$\mathrm{c}(\mathrm{ccc}(\mathrm{c} 1 \mathrm{Cl}) \mathrm{C})(\mathrm{c} 1) \mathrm{Cl}$

$\mathrm{C}(=\mathrm{CC}=\mathrm{C}(\mathrm{C} 1) \mathrm{C})(\mathrm{C} 1) \mathrm{C}(\mathrm{C}) \mathrm{C}$

$\mathrm{OC}(\mathrm{c}(\mathrm{cccc} 1) \mathrm{c} 1)$

$(\mathrm{C}(\mathrm{C}(\mathrm{C}=\mathrm{CC} 23) \mathrm{C} 2) \mathrm{C} 3) \mathrm{CCN}(\mathrm{CCCC} 4)$

$\mathrm{C} 4$

$\mathrm{Oc}(\mathrm{c}(\mathrm{cc}(\mathrm{c} 1) \mathrm{Cl}) \mathrm{Cc}(\mathrm{c}(\mathrm{O}) \mathrm{ccc} 2 \mathrm{Cl}) \mathrm{c} 2) \mathrm{c} 1$

$\mathrm{O}=\mathrm{P}(\mathrm{OCCCC})(\mathrm{OCCCC}) \mathrm{Oc}(\operatorname{cccc} 1) \mathrm{c} 1$

$\operatorname{COc} 1 \operatorname{ccc} 2 \mathrm{n}(\mathrm{C}(=\mathrm{O}) \mathrm{c} 3 \mathrm{ccc}(\mathrm{Cl}) \operatorname{cc} 3) \mathrm{c}(\mathrm{C})$

$\mathrm{c}(\mathrm{CC}(=\mathrm{O}) \mathrm{O}) \mathrm{c} 2 \mathrm{c} 1$

$\mathrm{Oc}(\mathrm{c}(\mathrm{c}(\mathrm{c}(\mathrm{c} 1 \mathrm{Cl}) \mathrm{Cl}) \mathrm{Cl}) \mathrm{Cl}) \mathrm{c} 1 \mathrm{O}$

$\mathrm{CN}(\mathrm{C}) \mathrm{CCC}=\mathrm{C} 2 \mathrm{c} 1 \mathrm{ccccc} 1 \mathrm{CO} 3 \mathrm{ccccc} 2$ 3

$\mathrm{OC} 2(\operatorname{CCN}(\operatorname{CCCC}(=0) \operatorname{c1ccc}(\mathrm{F}) \operatorname{cc} 1) \mathrm{C}$

$\mathrm{C} 2) \mathrm{c} 3 \mathrm{ccc}(\mathrm{Cl}) \mathrm{cc} 3$

$\mathrm{N}(=\mathrm{O})$

$(=\mathrm{O}) \mathrm{c}(\mathrm{ccc}(\mathrm{N}=\mathrm{Nc}(\operatorname{ccc}(\mathrm{N}(\mathrm{CCO}) \mathrm{CC}) \mathrm{c} 1) \mathrm{c}$

1)c2) $\mathrm{c} 2$

$\mathrm{N}(=\mathrm{O})$

$(=\mathrm{O}) \mathrm{c}(\mathrm{ccc}(\mathrm{N}=\mathrm{Nc}(\mathrm{c}(\mathrm{cc}(\mathrm{N}(\mathrm{CCO}) \mathrm{CCO}) \mathrm{c}$

1)C)c1) $\mathrm{c} 2 \mathrm{Cl}) \mathrm{c} 2$

$\mathrm{O}=\mathrm{C}(\mathrm{OC}(\mathrm{C}(=\mathrm{C}(\mathrm{C} 1(=\mathrm{O})) \mathrm{CC}=\mathrm{CC}=\mathrm{C}) \mathrm{C}$

C1 $) \mathrm{C}(\mathrm{C} 2(\mathrm{C}) \mathrm{C}) \mathrm{C} 2 \mathrm{C}=\mathrm{C}(\mathrm{C}(=\mathrm{O}) \mathrm{OC}) \mathrm{C}$

Oc1 $1 \mathrm{cc}(\mathrm{C}) \operatorname{cc} 1 \mathrm{n} 2 \mathrm{nc} 3 \operatorname{ccccc} 3 \mathrm{n} 2$

$\mathrm{n}(\mathrm{c}(\mathrm{c}(\mathrm{ccc} 1) \mathrm{cc} 2) \mathrm{c} 1) \mathrm{c} 2 \mathrm{c}(\mathrm{nc}(\mathrm{c}(\mathrm{ccc} 3) \mathrm{c} 4) \mathrm{c}$ 3) 4

$\mathrm{CC}(=\mathrm{O}) \mathrm{N}(\mathrm{C} 1) \mathrm{CCN}(\mathrm{C} 1) \mathrm{c}(\mathrm{c} 2) \operatorname{ccc}(\mathrm{c} 2) \mathrm{O}$

$\mathrm{CC}(\mathrm{CO} 3) \mathrm{OC} 3(\mathrm{Cn} 4 \mathrm{cncc} 4) \mathrm{c} 5 \mathrm{c}(\mathrm{Cl}) \mathrm{cc}(\mathrm{C}$ I)cc5

$\mathrm{BrCCCCCCC}$

$\mathrm{O}=\mathrm{C}(\mathrm{O}) \mathrm{c}(\mathrm{c}(\mathrm{Nc}(\mathrm{cccc} 1) \mathrm{c} 1) \mathrm{ccc} 2) \mathrm{c} 2$

$\mathrm{O}=\mathrm{C}(\mathrm{c}(\mathrm{c}(\mathrm{C}(=\mathrm{O}) \mathrm{c} 1 \mathrm{cccc} 2) \operatorname{ccc} 3 \mathrm{CC}) \mathrm{c} 3) \mathrm{c}$ 12

$\mathrm{CCCC}(=\mathrm{NOCC}) \mathrm{C} 1=\mathrm{C}(\mathrm{O}) \mathrm{CC}(\mathrm{CC}(\mathrm{C}) \mathrm{S}$

$\mathrm{CC}) \mathrm{CC} 1(=\mathrm{O})$

c(cccc1)(c1)CCCC

$\mathrm{s}(\mathrm{c}(\mathrm{c}(\mathrm{c} 1 \mathrm{cccc} 2) \mathrm{ccc} 3) \mathrm{c} 3) \mathrm{c} 12$

$\mathrm{N}(\mathrm{c}(\operatorname{ccc}(\mathrm{c} 1 \mathrm{ccc} 2) \mathrm{c} 2) \mathrm{c} 1) \mathrm{c}(\mathrm{cccc} 3) \mathrm{c} 3$

$\mathrm{C}(=\mathrm{CCC}(\mathrm{C} 12) \mathrm{C} 1(\mathrm{C}) \mathrm{C})(\mathrm{C} 2) \mathrm{C}$

$\operatorname{CCOP}(=\mathrm{S})$

$(\mathrm{OCC}) \mathrm{ON}=\mathrm{C}(\mathrm{C}(\# \mathrm{~N})) \mathrm{c} 1 \mathrm{ccccc} 1$

$\mathrm{O}=\mathrm{C}(\mathrm{OCC}(\mathrm{CC})$

$(\operatorname{COC}(=\mathrm{O}) \mathrm{C}(=\mathrm{C}) \mathrm{C}) \operatorname{coc}(=\mathrm{O}) \mathrm{C}(=\mathrm{C}) \mathrm{C})$

$\mathrm{C}(=\mathrm{C}) \mathrm{C}$

$\mathrm{CCCCOc} 2 \mathrm{cc}(\mathrm{C}(=\mathrm{O}) \mathrm{NCCN}(\mathrm{CC}) \mathrm{CC}) \mathrm{c} 1$

$\operatorname{ccccc} 1 \mathrm{n} 2$

$\mathrm{O}=\mathrm{C}(\mathrm{OC}) \mathrm{CCCCCCCCC}$

$\mathrm{S}(\mathrm{Sc}(\operatorname{cccc} 1) \mathrm{c} 1) \mathrm{c}(\operatorname{cccc} 2) \mathrm{c} 2$

$\mathrm{O}=\mathrm{C} 1 \mathrm{C}(\mathrm{C}(\mathrm{C})$

$(\mathrm{C}) \mathrm{C})=\mathrm{CC}(=\mathrm{O}) \mathrm{C}=\mathrm{C} 1 \mathrm{C}(\mathrm{C})(\mathrm{C}) \mathrm{C}$

$\mathrm{O}=\mathrm{C}(\mathrm{O}) \mathrm{CCCCCCCCCC}$

$\mathrm{c} 1 \mathrm{c}(\mathrm{C}(=\mathrm{O}) \mathrm{O}) \mathrm{c}(\mathrm{O}) \mathrm{ccc} 1 \mathrm{c} 2 \mathrm{c}(\mathrm{F}) \mathrm{cc}(\mathrm{F}) \mathrm{cc} 2$

$\mathrm{C}(=\mathrm{CC}(\mathrm{C} 1 \mathrm{C} 2) \mathrm{C} 1(\mathrm{C}) \mathrm{C})(\mathrm{C} 2) \mathrm{C}$

$\mathrm{Oc}(\mathrm{c}(\mathrm{cc}(\mathrm{c} 1 \mathrm{Cl}) \mathrm{Cl}) \mathrm{Cl}) \mathrm{c} 1 \mathrm{Cl}$

$\mathrm{S}(\mathrm{c}(\mathrm{cccc} 1) \mathrm{c} 1) \mathrm{c}(\mathrm{cccc} 2) \mathrm{c} 2$

$\mathrm{c}(\mathrm{c}(\mathrm{ccc} 1) \mathrm{cc}(\mathrm{c} 2 \mathrm{ccc} 3) \mathrm{c} 3)(\mathrm{c} 1) \mathrm{c} 2$

$\mathrm{O}=\mathrm{C}(\mathrm{OCC}=\mathrm{Cc}(\operatorname{cccc} 1) \mathrm{c} 1) \mathrm{C}=\mathrm{Cc}(\operatorname{cccc} 2$ ) $\mathrm{c} 2$

$\mathrm{c}(\mathrm{c}(\mathrm{c}(\mathrm{c}(\mathrm{c} 1) \mathrm{ccc} 2) \mathrm{c} 2) \mathrm{ccc} 3)(\mathrm{c} 1) \mathrm{c} 3$

$\mathrm{C}(=\mathrm{C}(\mathrm{C}) \mathrm{C})(\mathrm{CCC}(=\mathrm{C} 1) \mathrm{C}) \mathrm{C} 1$

$\mathrm{OC}(\mathrm{c}(\mathrm{cccc} 1) \mathrm{c} 1)$

(C(CCCC2)C2) CCN $(\mathrm{CCCC} 3) \mathrm{C} 3$

$\mathrm{O}=\mathrm{C}(\mathrm{OCCCC}) \mathrm{c}(\mathrm{c}(\mathrm{ccc} 1) \mathrm{C}(=\mathrm{O}) \mathrm{OCCC}$

\subsection{1}

4.22

4.22

4.24

4.25

4.25

4.05

4.33

4.35

3.83

4.75

4.95

$-0.16$

0.11

0.13

$-0.41$

0.50

0.70

3.68

4.38

4.56

3.95

4.51

4.28

$-0.53$

0.16

0.34

$-0.29$

0.26

0.03

\section{$\begin{array}{lllll}4.26 & 4.34 & 0.08 & 3.93 & -0.33\end{array}$}

$\begin{array}{lllll}4.27 & 4.11 & -0.16 & 3.74 & -0.53\end{array}$

$\begin{array}{lllll}4.27 & 4.23 & -0.04 & 4.25 & -0.02\end{array}$

$\begin{array}{lllll}4.29 & 3.61 & -0.68 & 4.26 & -0.03\end{array}$

$\begin{array}{lllll}4.29 & 3.99 & -0.30 & 4.08 & -0.21\end{array}$

$\begin{array}{lllll}4.30 & 4.20 & -0.10 & 3.70 & -0.60\end{array}$

4.30

4.20

$-0.10$

4.29

$-0.01$

4.30

4.33

0.03

3.74

$-0.56$

4.30

5.33

1.03

4.25

$-0.05$

4.31

3.00

-1.31
-0.25

2.64

4.31

$-1.67$

0.00

4.45

0.10

4.30

$-0.05$

4.12

$-0.24$

$-0.18$

4.40

4.19

0.04

4.38

0.01

3.69

$-0.68$

3.99

$-0.39$

3.89

$-0.49$

4.01

$-0.37$

4.34

$-0.04$

4.38

4.38

4.38

4.39

4.47

$-0.21$

4.38

0.00

0.15

4.61

4.53

$-0.74$

$-0.61$

4.39

4.50

0.11

3.00

$-1.39$

4.40

4.04

$-0.36$

3.79

$-0.61$

4.30

$-0.11$

4.58

0.17

4.41

4.31

$-0.10$

4.51

0.10

$-0.63$

4.42

4.51

0.09

4.49

0.07

$-1.33$

4.44

4.45

4.45

4.45

4.45

4.46

4.47

4.49

4.61

4.09

0.17

$-0.36$

$-0.16$

$-0.10$

0.38

3.26

$-1.18$

$-0.08$

4.35

4.83

4.36

4.56

$-0.09$

0.11

4.35

4.88

$-0.11$

0.41

4.62

0.17

4.55

3.82

0.09

5.27

0.78

4.93

0.44

4.61

$0.11 \quad 4.53$

0.03 
1,4-cyclohexadiene, 1-methyl-4-(1-methylethyl)2-octene

benzene, 1,3,5-tribromo-

2-propenoic acid, 2-methyl-, 2-ethylhexyl ester

1-pentene, 2,4,4-trimethyl-

9,10-anthracenedione, 1,4-diamino-2,3-dichloro-

benzene, tetrachloro-

1-decanol

benzene, (1-methylpropyl)-

1-octene

cyclohexene, 1-methyl-4-(1-methylethenyl)-

benzene, diethyl-

benzenamine, $\mathrm{N}, \mathrm{N}$-dimethyl-4-(phenylazo)phenol, 2,3,4,5-tetrachloro-6-methoxy-

phosphoric acid, triphenyl ester

benzenesulfonamide, 3-nitro-N-phenyl-4(phenylamino)-

benzene, 1,2,3,4-tetrachloro-

dodecanoic acid

1,1-biphenyl, 4-methyl-

1,4-pentanediamine, N,4-(7-chloro-4-quinolinyl)-

$\mathrm{N}, \mathrm{N}$-diethyl-, phosphate $(1: 2)$

benzene, 1,2,4,5-tetrachloro-

2,6-methano-3-benzazocin-8-ol, 1,2,3,4,5,6-

hexahydro-6,11-dimethyl-3-(3-methyl-2-butenyl)-

heptane

oxazole, 2,5-diphenyl-

benzene, (2-methylpropyl)-

$10 \mathrm{H}$-phenothiazine-10-propanamine, 2-methoxy-

$\mathrm{N}, \mathrm{N}$-beta-trimethyl-

piperidine, 4-(5H-dibenzo[a,d]cyclohepten-5-

ylidene)-1-methyl-

13-oxabicyclo[10.1.0]tridecane

$10 \mathrm{H}$-phenothiazine-10-propanamine, $\mathrm{N}, \mathrm{N}$-beta-

trimethyl-, 2,3-dihydroxybutanedioate (2:1)

cyclohexane, 1,2,3,4,5-pentabromo-6-chloro-

1,2-benzenedicarboxylic acid, butyl phenylmethyl

ester

1,3-benzodioxole, 5-[[2-(2-

butoxyethoxy)ethoxy]methyl]-6-propyl-

phenol, 5-chloro-2-(2,4-dichlorophenoxy)-

$10 \mathrm{H}$-phenothiazine-10-ethanamine, N,N-diethylalpha-methyl-

1,3-butadiene, 1,1,2,3,4,4-hexachloro-

cyclopropanecarboxylic acid, 2,2-dimethyl-3-(2-

methyl-1-propenyl)-, 2-methyl-4-oxo-3-(2-propenyl)-

2-cyclopenten-1-yl ester

benzene, 1,1-(1,2-ethanediyl)bis-

acetamide, $\mathrm{N}$-[5-[bis[2-(acetyloxy)ethyl]amino]-2-

[(2-bromo-4,6-dinitrophenyl)azo]-4-methoxyphenyl]-

$10 \mathrm{H}$-phenothiazine-10-ethanamine, $\mathrm{N}, \mathrm{N}$-alpha-

trimethyl-

benzene, 1,1-(1,2-ethenediyl)bis-

bicyclo[3.1.1] hept-2-ene, 2,6,6-trimethyl-

benzene, 1,2,3,4-tetrachloro-5,6-dimethoxy-

$10 \mathrm{H}$-phenothiazine, 2-chloro-10-[3-(4-methyl-1-

piperazinyl)propyl]-

pyrene

octane, 1-bromo-

phenol, 2,6-bis(1,1-dimethylethyl)-

1-propanamine, 3-(10,11-dihydro-5H-
C)c1

\begin{tabular}{|c|c|c|c|c|c|}
\hline$C(=C C C(=C 1) C)(C 1) C(C) C$ & 4.50 & 4.75 & 0.25 & 4.36 & -0.14 \\
\hline $\mathrm{C}(=\mathrm{CC}) \operatorname{CcCCC}$ & 4.51 & 4.06 & -0.45 & 4.59 & 0.08 \\
\hline $\mathrm{c}(\mathrm{cc}(\mathrm{cc} 1 \mathrm{Br}) \mathrm{Br})(\mathrm{c} 1) \mathrm{Br}$ & 4.51 & 4.66 & 0.15 & 4.42 & -0.09 \\
\hline $\mathrm{O}=\mathrm{C}(\mathrm{OCC}(\mathrm{CCCC}) \mathrm{CC}) \mathrm{C}(=\mathrm{C}) \mathrm{C}$ & 4.54 & 4.64 & 0.10 & 4.57 & 0.03 \\
\hline$C(=C)(C C(C)(C) C) C$ & 4.55 & 4.08 & -0.47 & 4.03 & -0. \\
\hline $\begin{array}{l}\mathrm{O}=\mathrm{C}(\mathrm{c}(\mathrm{c}(\mathrm{C}(=\mathrm{O}) \mathrm{c} 1 \mathrm{c}(\mathrm{N}) \mathrm{c}(\mathrm{c}(\mathrm{c} 2 \mathrm{~N}) \mathrm{Cl}) \mathrm{Cl}) \mathrm{c} \\
\mathrm{cc} 3) \mathrm{c} 3) \mathrm{c} 12\end{array}$ & 4.56 & 4.45 & -0.11 & 4.11 & -0. \\
\hline $\mathrm{Clc} 2 \mathrm{c}(\mathrm{cc}(\mathrm{cc} 2 \mathrm{Cl}) \mathrm{Cl}) \mathrm{Cl}$ & 4.56 & 4.57 & 0.01 & 4.63 & 0.0 \\
\hline occcсcсссC & 4.57 & 3.79 & -0.78 & 4.24 & -0. \\
\hline$c(\operatorname{cccc} 1)(c 1) C(C C) C$ & 4.57 & 3.94 & -0.63 & 4.36 & -0. \\
\hline$C(=C) \operatorname{cccccc}$ & 4.57 & 4.13 & -0.44 & 4.61 & 0.0 \\
\hline$C(=C C C(C(=C) C) C 1)(C 1) C$ & 4.57 & 4.83 & 0.26 & 4.50 & -0. \\
\hline $\mathrm{CCc} 1 \mathrm{ccc}(\mathrm{cc} 1) \mathrm{CC}$ & 4.58 & 4.07 & -0.51 & 4.36 & -0 \\
\hline $\mathrm{N}(=\mathrm{Nc}(\operatorname{ccc} 1) \mathrm{c} 1) \mathrm{c}(\operatorname{ccc}(\mathrm{N}(\mathrm{C}) \mathrm{C}) \mathrm{c} 2) \mathrm{c} 2$ & 4.58 & 4.29 & -0.29 & 4.63 & \\
\hline $\mathrm{COc} 1 \mathrm{c}(\mathrm{O}) \mathrm{c}(\mathrm{Cl}) \mathrm{c}(\mathrm{Cl}) \mathrm{c}(\mathrm{Cl}) \mathrm{c} 1 \mathrm{Cl}$ & 4.59 & 3.92 & -0.67 & 4.44 & \\
\hline $\begin{array}{l}\mathrm{O}=\mathrm{P}(\mathrm{Oc}(\mathrm{cccc} 1) \mathrm{c} 1) \\
(\mathrm{Oc}(\mathrm{cccc} 2) \mathrm{c} 2) \mathrm{Oc}(\mathrm{cccc} 3) \mathrm{c} 3\end{array}$ & 4.59 & 4.70 & 0.11 & 4.16 & \\
\hline $\mathrm{O}=\mathrm{S}(=\mathrm{O})$ & 4.60 & 4.33 & -0.27 & 3.79 & \\
\hline
\end{tabular}

$(\mathrm{Nc}(\mathrm{cccc} 1) \mathrm{c} 1) \mathrm{c}(\mathrm{ccc}(\mathrm{Nc}(\mathrm{cccc} 2) \mathrm{c} 2) \mathrm{c} 3 \mathrm{~N}$

$(=0)(=0)) c 3$

$\mathrm{c}(\mathrm{c}(\mathrm{c}(\mathrm{c}(\mathrm{c} 1) \mathrm{Cl}) \mathrm{Cl}) \mathrm{Cl})(\mathrm{c} 1) \mathrm{Cl}$

$\mathrm{O}=\mathrm{C}(\mathrm{O}) \mathrm{CCCCCCCCCCC}$

$\mathrm{c} 1 \mathrm{ccccc} 1 \mathrm{c} 2 \mathrm{ccc}(\mathrm{C}) \mathrm{cc} 2$

c12nccc $(\mathrm{NC}(\mathrm{C}) \mathrm{CCCN}(\mathrm{CC}) \mathrm{CC}) \mathrm{c} 1 \mathrm{ccc}($ $\mathrm{Cl}) \mathrm{C} 2$

$\mathrm{c}(\mathrm{c}(\mathrm{cc}(\mathrm{c} 1 \mathrm{Cl}) \mathrm{Cl}) \mathrm{Cl})(\mathrm{c} 1) \mathrm{Cl}$

$\mathrm{CC} 2 \mathrm{C} 3 \mathrm{Cc} 1 \mathrm{ccc}(\mathrm{O}) \mathrm{cc} 1 \mathrm{C} 2(\mathrm{C}) \mathrm{CCN} 3 \mathrm{CC}$

$\mathrm{C}(\mathrm{CCCCC}) \mathrm{C}$

(c(c(ccc1)c1)cn2)c2c(ccc3) 3

$\mathrm{c}(\mathrm{cccc} 1)(\mathrm{c} 1) \mathrm{CC}(\mathrm{C}) \mathrm{C}$

$\mathrm{c} 1 \mathrm{ccc} 2 \mathrm{Sc} 3 \operatorname{ccc}(\mathrm{OC}) \operatorname{cc} 3 \mathrm{~N}(\mathrm{CC}(\mathrm{C}) \mathrm{CN}(\mathrm{C}$

)C) 2 c1

$\mathrm{N}(\mathrm{CCC}(=\mathrm{C}(\mathrm{c}(\mathrm{c}(\mathrm{C}=\mathrm{C} \mathrm{c} 1 \mathrm{ccc} 2) \mathrm{ccc} 3) \mathrm{c} 3)$

c12)C4)(C4)C

$\mathrm{C} 10 \mathrm{C} 1 \mathrm{CCCCCCCCC}=\mathrm{C}$

$\mathrm{c} 12 \mathrm{~N}(\mathrm{CC}(\mathrm{C}) \mathrm{CN}(\mathrm{C}) \mathrm{C}) \mathrm{c} 3 \mathrm{ccccc} 3 \mathrm{Sc} 1 \mathrm{cc}$

$\mathrm{CC} 2$

$\mathrm{BrC}(\mathrm{C}(\mathrm{C}(\mathrm{Br}) \mathrm{C}(\mathrm{Br}) \mathrm{C} 1 \mathrm{Br}) \mathrm{Cl}) \mathrm{C} 1 \mathrm{Br}$

$\mathrm{O}=\mathrm{C}(\mathrm{OCc}(\operatorname{ccc} 1) \mathrm{c} 1) \mathrm{c}(\mathrm{c}(\operatorname{ccc} 2) \mathrm{C}(=\mathrm{O}) \mathrm{O}$

$\mathrm{CCCC}) \mathrm{c} 2$

$\mathrm{O}(\mathrm{c}(\mathrm{c}(\mathrm{O} 1) \mathrm{cc}(\mathrm{c} 2 \mathrm{CCC}) \mathrm{COCCOCCOC}$

$\mathrm{CCC}) \mathrm{C} 2) \mathrm{C} 1$

$\mathrm{O}(\mathrm{c}(\mathrm{c}(\mathrm{O}) \mathrm{cc}(\mathrm{c} 1) \mathrm{Cl}) \mathrm{c} 1) \mathrm{c}(\mathrm{c}(\mathrm{cc}(\mathrm{c} 2) \mathrm{Cl}) \mathrm{Cl})$

$\mathrm{c} 2$

$\mathrm{c} 1 \mathrm{cc} 2 \mathrm{Sc} 3 \operatorname{ccccc} 3 \mathrm{~N}(\mathrm{CC}(\mathrm{C}) \mathrm{N}(\mathrm{CC}) \mathrm{CC}) \mathrm{c}$

2cc1

$\mathrm{C}(=\mathrm{C}(\mathrm{C}(=\mathrm{C}(\mathrm{Cl}) \mathrm{Cl}) \mathrm{Cl}) \mathrm{Cl})(\mathrm{Cl}) \mathrm{Cl}$

4.60

4.63

4.63

4.64

4.64

4.66

4.67

4.68

4.68

4.69

4.70

4.71

4.72

4.73

4.57

5.00

4.30

4.50

4.57

5.03

3.78

3.74

3.94

5.06

5.17

$-0.03$

0.40

$-0.33$

$-0.13$

4.62

5.13

4.42

5.28

$-0.07$

0.39

4.61

4.44

$-0.88$

4.33

4.03

$-0.74$

0.38

4.13

4.84

0.48

5.02

0.33

4.66

$-0.04$

4.78

4.82

0.08

4.71

$-0.01$

4.00

4.54

$-0.72$

4.84

0.11

4.29

$-0.46$

3.07

$-1.68$

4.66

$-0.10$

5.53

0.77

5.47

0.70

5.75

0.98

4.72

$-0.06$

4.86

0.08

$\mathrm{O}=\mathrm{C}(\mathrm{OC}(\mathrm{C}(=\mathrm{C}(\mathrm{C} 1(=\mathrm{O})) \mathrm{CC}=\mathrm{C}) \mathrm{C}) \mathrm{C} 1)$

4.78

5.52

0.74

3.58

$-1.20$

$\mathrm{c}(\mathrm{cccc} 1)(\mathrm{c} 1) \mathrm{CCc}(\operatorname{ccc} 2) \mathrm{c} 2$

4.79

4.74

$-0.05$

4.74

$\mathrm{O}=\mathrm{C}(\mathrm{OCCN}(\mathrm{c}(\mathrm{c}(\mathrm{OC}) \mathrm{cc}(\mathrm{N}=\mathrm{Nc}(\mathrm{c}(\mathrm{cc}(\mathrm{N})$

4.80

5.04

0.24

4.46

$-0.05$

$(=\mathrm{O})) \mathrm{c} 2 \mathrm{NC}(=\mathrm{O}) \mathrm{C}) \mathrm{c} 2) \mathrm{CCOC}(=\mathrm{O}) \mathrm{C}) \mathrm{C}$

$\mathrm{c} 1 \mathrm{ccc} 2 \mathrm{Sc} 3 \operatorname{ccccc} 3 \mathrm{~N}(\mathrm{CC}(\mathrm{C}) \mathrm{N}(\mathrm{C}) \mathrm{C}) \mathrm{c} 2 \mathrm{c}$ 1

$\mathrm{c}(\mathrm{cccc} 1)(\mathrm{c} 1) \mathrm{C}=\mathrm{Cc}(\mathrm{cccc} 2) \mathrm{c} 2$

4.81

4.49

$-0.32$

4.52

$-0.29$

$\mathrm{C}(\mathrm{C}(\mathrm{CC} 1 \mathrm{C} 2) \mathrm{C} 1(\mathrm{C}) \mathrm{C})(=\mathrm{C} 2) \mathrm{C}$

4.52

$-0.29$

4.58

3.66

$-0.64$

4.64

4.67

$-1.17$

$\mathrm{CN} 4 \mathrm{CCN}(\mathrm{CCCN} 2 \mathrm{c} 1 \mathrm{ccccc} 1 \mathrm{Sc} 3 \mathrm{ccc}(\mathrm{Cl}$

4.86 )cc23)CC4

$\mathrm{c}(\mathrm{c}(\mathrm{c}(\mathrm{cc} 1) \mathrm{ccc} 2) \mathrm{c} 2 \mathrm{cc} 3)(\mathrm{c} 1 \mathrm{ccc} 4) \mathrm{c} 34$

$\mathrm{BrCCCCCCCC}$

4.88

4.22

$-0.09$

$-0.22$

$-0.21$

4.93

0.05

5.19

0.31

$\mathrm{Oc}(\mathrm{c}(\mathrm{ccc} 1) \mathrm{C}(\mathrm{C})(\mathrm{C}) \mathrm{C}) \mathrm{c} 1 \mathrm{C}(\mathrm{C})(\mathrm{C}) \mathrm{C}$

4.61

$-0.28$

4.91

0.02

$\mathrm{CN}(\mathrm{C}) \mathrm{CCC}=\mathrm{C} 2 \mathrm{c} 1 \mathrm{ccccc} 1 \mathrm{CCc} 3 \mathrm{ccccc} 2$

4.92

4.48
4.95

0.03

$5.10-0.18$ 
dibenzo[a,d]cyclohepten-5-ylidene)-N,N-dimethyl1,1-biphenyl, 4-bromophosphorothioic acid, o,o-diethyl o-(3,5,6-trichloro2-pyridinyl) ester

1H-indene-1,3(2H)-dione, 2-(3-hydroxy-2quinolinyl)-

decane

10H-phenothiazine, 10-[3-(4-methyl-1-

piperazinyl)propyl]-2-(trifluoromethyl)-

1,3-cyclopentadiene, 1,2,3,4,5,5-hexachloro-

anthracene, 9-methyl-

benzene, 1,1'-(2,2,2-trichloroethylidene)bis[4-

methoxy-]

phenol, 2,6-bis(1,1-dimethylethyl)methyl-

benzene, hexamethyl-

phenol, pentachloro-

benzoic acid, 2-[(2,3-dimethylphenyl)amino]-

1-dodecanol

benzene, 1-(1,1-dimethylethyl)-4-methyl-

benzene, pentachloro-

octane

phenol, 2,4-bis(1,1-dimethylethyl)-

9,10-anthracenedione, 1,4-diamino-2,3-diphenoxy-

$5 \mathrm{H}$-dibenz[b,f]azepine-5-propanamine, 3-chloro-

10,11-dihydro-N,N-dimethyl-

1,1-biphenyl, (1-methylethyl)-

benzene, 1,1',1"-(chloromethylidyne)tris-

benzenamine, 2,6-dinitro-N,N-dipropyl-4-

(trifluoromethyl)-

acetamide, N-[2-[(2-bromo-4,6-dinitrophenyl)azo]-

5-[(2-cyanoethyl)-2-propenylamino]-4-

methoxyphenyl]-

$10 \mathrm{H}$-phenothiazine-10-propanamine, 2-chloro- $\mathrm{N}, \mathrm{N}$ dimethyl-

dodecanoic acid, methyl ester

1,3-benzenedicarbonitrile, 2-[[4-[[2-

(acetyloxy)ethyl]butylamino]-2-methylphenyl]azo]-5-

nitro-

peroxide, bis(1-methyl-1-phenylethyl)

cyclohexane, 1-methyl-4-(1-methylethyl)-

phenol, isononyl-

1,2-benzenedicarboxylic acid, dipentyl ester

retinol

phosphine, triphenyl-

1-decene

dodecanoic acid, ethyl ester

benzene, hexachloro-

phosphoric acid, 2-ethylhexyl diphenyl ester

phenol, 4-nonyl-

benz[a]anthracene, 7,12-dimethyl-

chrysene

cyclopropanecarboxylic acid, 2,2-dimethyl-3-(2-

methyl-1-propenyl)-, 2-methyl-4-oxo-3-(2,4-

pentadienyl)-2-cyclopenten-1-yl ester

10H-phenothiazine, 10-[2-(1-methyl-2-

piperidinyl)ethyl]-2-(methylthio)-

terphenyl

1-tetradecanol

phenol, 2,4,6-tris(1,1-dimethylethyl)-
3

$\mathrm{c}(\operatorname{ccc}(\mathrm{c}(\operatorname{cccc} 1) \mathrm{c} 1) \mathrm{c} 2)(\mathrm{c} 2) \mathrm{Br}$

$\mathrm{CCOP}(=\mathrm{S})$
$(\mathrm{OCC}) \mathrm{Oc} 1 \mathrm{nc}(\mathrm{Cl}) \mathrm{c}(\mathrm{Cl}) \mathrm{cc} 1 \mathrm{Cl}$

$\mathrm{O}=\mathrm{C}(\mathrm{c}(\mathrm{c}(\mathrm{C} 1(=\mathrm{O})) \mathrm{ccc} 2) \mathrm{c} 2) \mathrm{C} 1 \mathrm{c}(\mathrm{nc}(\mathrm{c}(\mathrm{c}$

cc3)c4)c3)c4O

$\mathrm{C}(\mathrm{CCCCCCCC)C}$

$\mathrm{CN} 4 \mathrm{CCN}(\mathrm{CCCN} 2 \mathrm{c} 1 \mathrm{ccccc} 1 \mathrm{Sc} 3 \mathrm{ccc}(\mathrm{cc}$

23) $\mathrm{C}(\mathrm{F})(\mathrm{F}) \mathrm{F}) \mathrm{CC} 4$

$\mathrm{C}(=\mathrm{C}(\mathrm{C}(=\mathrm{C} 1 \mathrm{Cl}) \mathrm{Cl}) \mathrm{Cl})(\mathrm{C} 1(\mathrm{Cl}) \mathrm{Cl}) \mathrm{Cl}$

$\mathrm{c}(\mathrm{c}(\mathrm{c}(\mathrm{c}(\mathrm{c} 1 \mathrm{ccc} 2) \mathrm{c} 2) \mathrm{C}) \mathrm{ccc} 3)(\mathrm{c} 3) \mathrm{c} 1$

$\mathrm{COc} 1 \mathrm{ccc}(\mathrm{cc} 1) \mathrm{C}(\mathrm{c} 2 \mathrm{ccc}(\mathrm{OC}) \mathrm{cc} 2) \mathrm{C}(\mathrm{Cl})$

(Cl)Cl

$\mathrm{CC}(\mathrm{c} 1 \mathrm{cc}(\operatorname{cc}(\mathrm{c} 1 \mathrm{O}) \mathrm{C}(\mathrm{C})(\mathrm{C}) \mathrm{C}) \mathrm{C})(\mathrm{C}) \mathrm{C}$

$\mathrm{c}(\mathrm{c}(\mathrm{c}(\mathrm{c}(\mathrm{c} 1 \mathrm{C}) \mathrm{C}) \mathrm{C}) \mathrm{C})(\mathrm{c} 1 \mathrm{C}) \mathrm{C}$

$\mathrm{Oc}(\mathrm{c}(\mathrm{c}(\mathrm{c}(\mathrm{c} 1 \mathrm{Cl}) \mathrm{Cl}) \mathrm{Cl}) \mathrm{Cl}) \mathrm{c} 1 \mathrm{Cl}$

$\mathrm{OC}(=\mathrm{O}) \mathrm{c} 1 \mathrm{ccccc} 1 \mathrm{Nc} 2 \mathrm{c}(\mathrm{C}) \mathrm{c}(\mathrm{C}) \mathrm{ccc} 2$

$\mathrm{OCCCCCCCCCCCC}$

$\mathrm{c}(\operatorname{ccc}(\mathrm{c} 1) \mathrm{C})(\mathrm{c} 1) \mathrm{C}(\mathrm{C})(\mathrm{C}) \mathrm{C}$

$\mathrm{c}(\mathrm{c}(\mathrm{c}(\mathrm{c}(\mathrm{c} 1 \mathrm{Cl}) \mathrm{Cl}) \mathrm{Cl}) \mathrm{Cl})(\mathrm{c} 1) \mathrm{Cl}$

$\mathrm{C}(\mathrm{CCCCCC}) \mathrm{C}$

$\mathrm{Oc}(\mathrm{c}(\mathrm{cc}(\mathrm{c} 1) \mathrm{C}(\mathrm{C})(\mathrm{C}) \mathrm{C}) \mathrm{C}(\mathrm{C})(\mathrm{C}) \mathrm{C}) \mathrm{c} 1$

$\mathrm{O}=\mathrm{C}(\mathrm{c}(\mathrm{c}(\mathrm{C}(=\mathrm{O}) \mathrm{c} 1 \mathrm{c}(\mathrm{N}) \mathrm{c}(\mathrm{Oc}(\mathrm{cccc} 2) \mathrm{c} 2)$

$\mathrm{c}(\mathrm{Oc}(\mathrm{cccc} 3) \mathrm{c} 3) \mathrm{c} 4 \mathrm{~N}) \mathrm{ccc5}) \mathrm{c5}) \mathrm{c} 14$

$\mathrm{c} 12 \mathrm{~N}(\mathrm{CCCN}(\mathrm{C}) \mathrm{C}) \mathrm{c} 3 \mathrm{cc}(\mathrm{Cl}) \mathrm{ccc} 3 \mathrm{CCc} 1$

cccc2

$\mathrm{CC}(\mathrm{c} 1 \mathrm{c}(\operatorname{cccc} 1) \mathrm{c} 2 \mathrm{ccccc} 2) \mathrm{C}$

$\mathrm{c}(\mathrm{cccc} 1)(\mathrm{c} 1) \mathrm{C}(\mathrm{c}(\mathrm{cccc} 2) \mathrm{c} 2)$

(c(cccc3)c3) Cl

$\operatorname{CCCN}(\mathrm{CCC}) \operatorname{c} 1 \mathrm{c}(\operatorname{cc}(\operatorname{cc} 1 \mathrm{~N}(=\mathrm{O})$

$(=\mathrm{O})) \mathrm{C}(\mathrm{F})(\mathrm{F}) \mathrm{F}) \mathrm{N}(=\mathrm{O})(=\mathrm{O})$

$\mathrm{N}(=\mathrm{O})(=\mathrm{O}) \operatorname{c} 1 \mathrm{cc}(\mathrm{N}(=\mathrm{O})$

$(=\mathrm{O})) \mathrm{cc}(\mathrm{Br}) \mathrm{c} 1 \mathrm{~N}=\mathrm{Nc} 2 \mathrm{cc}(\mathrm{OC}) \mathrm{c}(\mathrm{N}(\mathrm{CC}=$

C) $\operatorname{CCC}(\# \mathrm{~N})) \operatorname{cc} 2 \mathrm{NC}(=\mathrm{O}) \mathrm{C}$

$\mathrm{CN}(\mathrm{C}) \mathrm{CCCN} 2 \mathrm{c} 1 \mathrm{ccccc} 1 \mathrm{Sc} 3 \mathrm{ccc}(\mathrm{Cl}) \mathrm{cc}$ 23

$\mathrm{O}=\mathrm{C}(\mathrm{OC}) \mathrm{CCCCCCCCCCC}$

$\mathrm{O}=\mathrm{C}(\mathrm{OCCN}(\mathrm{c}(\operatorname{ccc}(\mathrm{N}=\mathrm{Nc}(\mathrm{c}(\mathrm{C}(\# \mathrm{~N})) \mathrm{cc}($

4.96

4.96

4.65

4.66

$-0.31$

4.57

$-0.39$

5.00

4.83

$-0.30$

5.15

0.19

5.01

5.25

$-0.17$

3.29

$-1.71$

5.11

0.24

5.87

0.86

4.63

0.08

4.87

$-0.16$

4.85

$-0.19$

5.08

5.67

0.59

$5.06-0.01$

$5.12 \quad 0.04$

$5.25 \quad 0.15$

$\begin{array}{lllll}5.10 & 5.03 & -0.07 & 5.25 & 0.15 \\ 5.11 & 5.28 & 0.17 & 4.71 & -0.40\end{array}$

$\begin{array}{lllll}5.12 & 4.74 & -0.38 & 4.99 & -0.13\end{array}$

$\begin{array}{lllll}5.12 & 5.28 & 0.16 & 4.58 & -0.54\end{array}$

$\begin{array}{lllll}5.13 & 4.77 & -0.36 & 5.36 & 0.23\end{array}$

$\begin{array}{lllll}5.17 & 4.45 & -0.72 & 4.44 & -0.73\end{array}$

$\begin{array}{lllll}5.17 & 5.22 & 0.05 & 5.22 & 0.05\end{array}$

$\begin{array}{lllll}5.18 & 4.27 & -0.91 & 4.73 & -0.45\end{array}$

$\begin{array}{lllll}5.19 & 5.33 & 0.14 & 4.91 & -0.28\end{array}$

$\begin{array}{lllll}5.19 & 5.59 & 0.40 & 4.93 & -0.26\end{array}$

5.19

5.65

0.46

5.04

$-0.15$

5.20

5.21

0.01

5.25

0.05

$5.25 \quad 5.58$

0.33

5.63

0.38

$\begin{array}{lllll}5.34 & 5.31 & -0.03 & 5.09 & -0.25\end{array}$

$\begin{array}{lllll}5.40 & 5.34 & -0.06 & 5.32 & -0.08\end{array}$

5.41

5.20

$-0.21$

5.18

$-0.23$

5.41

5.28

$-0.13$

5.51

0.10

$(=\mathrm{O}) \mathrm{c} 1) \mathrm{c} 1 \mathrm{C}(\# \mathrm{~N})) \mathrm{c} 2 \mathrm{C}) \mathrm{c} 2) \mathrm{CCCC} \mathrm{C}$

$\mathrm{O}(\mathrm{OC}(\mathrm{c}(\mathrm{cccc} 1) \mathrm{c} 1)(\mathrm{C}) \mathrm{C}) \mathrm{C}(\mathrm{c}(\mathrm{cccc} 2) \mathrm{c} 2)$

(C)C

Oc1cc(ccc1)CCCCCCCCC

$\mathrm{O}=\mathrm{C}(\mathrm{OCCCCC}) \mathrm{c}(\mathrm{c}(\operatorname{ccc} 1) \mathrm{C}(=\mathrm{O}) \mathrm{OCC}$

$\mathrm{CCC}) \mathrm{c} 1$

$\mathrm{OCC}=\mathrm{C}(\mathrm{C}=\mathrm{CC}=\mathrm{C}(\mathrm{C}=\mathrm{CC}(=\mathrm{C}(\mathrm{CCC} 1)$

$\mathrm{C}) \mathrm{C} 1(\mathrm{C}) \mathrm{C}) \mathrm{C}) \mathrm{C}$

$c(P(c(\operatorname{cccc} 1) c 1) c(\operatorname{cccc} 2) c 2)(\operatorname{cccc} 3) c 3$

$\mathrm{C}(=\mathrm{C}) \mathrm{CCCCCCCC}$

$\mathrm{O}=\mathrm{C}(\mathrm{OCC}) \operatorname{cccccccccc}$

$\mathrm{c}(\mathrm{c}(\mathrm{c}(\mathrm{c}(\mathrm{c} 1 \mathrm{Cl}) \mathrm{Cl}) \mathrm{Cl}) \mathrm{Cl})(\mathrm{c} 1 \mathrm{Cl}) \mathrm{Cl}$

$\mathrm{O}=\mathrm{P}(\mathrm{Oc} 1 \mathrm{ccccc} 1)$

5.50

5.83

0.33

5.38

$-0.12$

Oc2cccc2)OCC(CC)CCCC

$\mathrm{Oc}(\operatorname{ccc}(\mathrm{c} 1) \operatorname{CCCCCCCCC}) \mathrm{c} 1$

$\mathrm{c}(\mathrm{c}(\mathrm{c}(\mathrm{c}(\mathrm{c} 1) \mathrm{ccc} 2) \mathrm{c} 2) \mathrm{c}(\mathrm{c}(\mathrm{c} 3 \mathrm{ccc} 4) \mathrm{c} 4) \mathrm{C})$

(c3C)c1

c1ccc2 $c c c 3 c 4 c c c c c 4 c c c 3 c 2 c 1$

$\mathrm{CC}(\mathrm{C})=\mathrm{CC} 2 \mathrm{C}(\mathrm{C}(=\mathrm{O}) \mathrm{OC} 1 \mathrm{CC}(=\mathrm{O}) \mathrm{C}(=$

5.50

5.88

0.38

5.43

$-0.07$

4.92

$-0.64$

4.95

$-0.61$

5.61

5.99

0.38

6.07

0.46

$\begin{array}{lll}-0.03 & 5.55 & -0.07\end{array}$

$\mathrm{C} 1 \mathrm{C}) \mathrm{CC}=\mathrm{CC}=\mathrm{C}) \mathrm{C} 2(\mathrm{C}) \mathrm{C}$

$\mathrm{CSc} 4 \operatorname{ccc} 3 \mathrm{Sc} 1 \operatorname{ccccc} 1 \mathrm{~N}(\mathrm{CCC} 2 \mathrm{CCCCN}$

2C)c $3 \mathrm{c} 4$

c1 $\operatorname{ccc}(\mathrm{cc} 1) \mathrm{c} 2 \mathrm{ccc}(\mathrm{cc} 2) \mathrm{c} 3 \mathrm{ccccc} 3$

5.68

7.62

1.94

6.38

0.70

5.02

$-0.67$

5.46

$-0.23$

5.70

5.12

$-0.58$

5.63

5.95

$-0.07$

5.86

$5.70-0.03$

5.73

6.30

0.57

5.30

-0.03
-0.43

5.76

5.99

0.23

6.09

0.33

6.62

0.82

6.61

$5.52 \quad-0.29$

5.71

$-0.10$

OCCCCCCCCCCCCCC

$\mathrm{Oc}(\mathrm{c}(\mathrm{cc}(\mathrm{c} 1) \mathrm{C}(\mathrm{C})(\mathrm{C}) \mathrm{C}) \mathrm{C}(\mathrm{C})$

$(\mathrm{C}) \mathrm{C}) \mathrm{c} 1 \mathrm{C}(\mathrm{C})(\mathrm{C}) \mathrm{C}$
5.81

6.28

0.38

5.10

$-0.80$

5.90

6.45

0.55

5.93

0.03

5.52

$-0.51$

5.70

$-0.33$

6.03

5.75

6.39

$-0.28$

0.33

6.21

6.28 
dodecane

tetradecanoic acid

octanoic acid, heptyl ester

heptanoic acid, octyl ester

decanoic acid, pentyl ester

butanoic acid, undecyl ester

benzo[a]pyrene

cyclopropanecarboxylic acid, 2,2-dimethyl-3-(2-

methyl-1-propenyl)-, [5-(phenylmethyl)-3-

furanyl]methyl ester

chlordane

phenol, 2,2-methylenebis[6-(1,1-dimethylethyl)-4methyl-

1,1'-biphenyl, 2,2,4,4'-tetrachloro

$9,12,15$-octadecatrienoic acid, methyl ester

2H-benzimidazol-2-one, 1-[1-[4,4-bis(4fluorophenyl)butyl]-4-piperidinyl]-1,3-dihydro-

retinoic acid

tetradecanoic acid, methyl ester

benz[j]aceanthrylene, 1,2-dihydro-3-methyl-

9,12,15-octadecatrienoic acid

octanoic acid, octyl ester

dodecanoic acid, butyl ester

9-hexadecenoic acid

1,2-benzenedicarboxylic acid, dihexyl ester

1,2-benzenedicarboxylic acid, butyl octyl ester

9,12-octadecadienoic acid, methyl ester

arachidonic acid

9,12-octadecadienoic acid

octadecadienoic acid

hexadecanoic acid

tetradecane

hexadecanoic acid, methyl ester

9-octadecenoic acid, methyl ester

phenol, 2,2'-methylenebis[3,4,6-trichloro-]

1,2-benzenedicarboxylic acid, bis(2-ethylhexyl) ester

9-octadecenoic acid

11-octadecenoic acid

phenol, 4-dodecyl-

1,2-benzenedicarboxylic acid, dioctyl ester

1,2-benzenedicarboxylic acid, decyl hexyl ester

octadecanoic acid

octadecanoic acid, methyl ester

benzene, dodecyl-

eicosanoic acid

\begin{tabular}{|c|c|c|c|c|c|}
\hline $\mathrm{C}(\operatorname{ccccccc} \mathrm{ccc}) \mathrm{C}$ & 6.10 & 6.23 & 0.13 & 6.42 & 0.32 \\
\hline $\mathrm{O}=\mathrm{C}(\mathrm{O}) \operatorname{ccccccccccc} \mathrm{C}$ & 6.11 & 5.98 & -0.13 & 6.10 & -0.01 \\
\hline $\operatorname{cccccccc}(=0) 0 \operatorname{Occccc} c$ & 6.11 & 6.27 & 0.16 & 6.09 & -0.02 \\
\hline $\operatorname{ccccccccoc}(=0) \operatorname{ccccc} c$ & 6.11 & 6.27 & 0.16 & 6.10 & -0.01 \\
\hline $\operatorname{cccccccccc}(=0)$ Occccc & 6.11 & 6.27 & 0.16 & 6.11 & 0.00 \\
\hline $\operatorname{cccccccccccoc}(=0) \operatorname{ccc}$ & 6.11 & 6.27 & 0.16 & 6.28 & 0.17 \\
\hline $\begin{array}{l}\mathrm{c}(\mathrm{c}(\mathrm{c}(\mathrm{cc} 1) \mathrm{ccc} 2) \mathrm{c} 2 \mathrm{cc} 3) \\
(\mathrm{c} 3 \mathrm{cc}(\mathrm{c} 4 \mathrm{ccc} 5) \mathrm{c} 5) \mathrm{c} 14\end{array}$ & 6.13 & 6.11 & -0.02 & 6.39 & 0.26 \\
\hline $\begin{array}{l}\mathrm{CC}(\mathrm{C})=\mathrm{CC} 3 \mathrm{C}(\mathrm{C}(=\mathrm{O}) \mathrm{OC} \mathrm{c} 2 \mathrm{coc}(\mathrm{Cc} 1 \mathrm{cc} \\
\operatorname{ccc} 1) \mathrm{c} 2) \mathrm{C} 3(\mathrm{C}) \mathrm{C}\end{array}$ & 6.14 & 7.11 & 0.97 & 5.38 & -0.76 \\
\hline $\begin{array}{l}\mathrm{ClC}(\mathrm{C}(\mathrm{Cl}) \mathrm{C} 1) \mathrm{C}(\mathrm{C} 1 \mathrm{C} 2(\mathrm{Cl}) \mathrm{C}=3 \mathrm{Cl}) \mathrm{C}(\mathrm{Cl}) \\
(\mathrm{C} 3 \mathrm{Cl}) \mathrm{C} 2(\mathrm{Cl}) \mathrm{Cl}\end{array}$ & 6.22 & 6.26 & 0.04 & 6.02 & -0.20 \\
\hline $\begin{array}{l}\mathrm{Oc}(\mathrm{c}(\mathrm{cc}(\mathrm{c} 1) \mathrm{C}) \mathrm{Cc}(\mathrm{c}(\mathrm{O}) \mathrm{c}(\mathrm{cc} 2 \mathrm{C}) \mathrm{C}(\mathrm{C}) \\
(\mathrm{C}) \mathrm{C}) \mathrm{c} 2) \mathrm{c} 1 \mathrm{C}(\mathrm{C})(\mathrm{C}) \mathrm{C}\end{array}$ & 6.25 & 7.97 & 1.72 & 5.89 & -0.36 \\
\hline $\mathrm{Clc} 2 \mathrm{c}(\mathrm{ccc}(\mathrm{c} 2) \mathrm{Cl}) \mathrm{c} 1 \mathrm{c}(\mathrm{cc}(\mathrm{cc} 1) \mathrm{Cl}) \mathrm{Cl}$ & 6.29 & 6.34 & 0.05 & 6.23 & -0.06 \\
\hline $\begin{array}{l}\mathrm{O}=\mathrm{C}(\mathrm{OC}) \mathrm{CCCCCCCC}=\mathrm{CCC}=\mathrm{CCC}= \\
\mathrm{CCC}\end{array}$ & 6.29 & 7.59 & 1.30 & 6.62 & 0.33 \\
\hline $\begin{array}{l}\mathrm{C}(\mathrm{c} 1 \mathrm{ccc}(\mathrm{F}) \mathrm{cc} 1) \\
(\mathrm{c} 2 \mathrm{ccc}(\mathrm{F}) \operatorname{cc} 2) \mathrm{CCCN}(\mathrm{CC} 3) \mathrm{CCC} 3 \mathrm{~N} 4 \mathrm{C}( \\
=0) \mathrm{Nc} 5 \mathrm{ccccc} 45\end{array}$ & 6.30 & 6.23 & -0.07 & 6.36 & 0.06 \\
\hline $\begin{array}{l}\mathrm{O}=\mathrm{C}(\mathrm{O}) \mathrm{C}=\mathrm{C}(\mathrm{C}=\mathrm{CC}=\mathrm{C}(\mathrm{C}=\mathrm{CC}(=\mathrm{C}(\mathrm{CC} \\
\mathrm{C} 1) \mathrm{C}) \mathrm{C} 1(\mathrm{C}) \mathrm{C}) \mathrm{C}) \mathrm{C}\end{array}$ & 6.30 & 7.85 & 1.55 & 5.66 & -0.64 \\
\hline $\mathrm{O}=\mathrm{C}(\mathrm{OC}) \mathrm{CcC} \operatorname{ccc} \mathrm{Ccc} C \mathrm{C}$ & 6.41 & 6.27 & -0.14 & 6.38 & -0.03 \\
\hline $\begin{array}{l}c(c(\operatorname{ccc} 1 \mathrm{C}) \mathrm{cc}(\mathrm{c} 2 \mathrm{ccc} 3 \operatorname{ccc} 4) \mathrm{c} 34) \\
(\mathrm{c} 1 \mathrm{CC} 5) \mathrm{c} 25\end{array}$ & 6.42 & 7.05 & 0.63 & 6.49 & 0.07 \\
\hline $\begin{array}{l}\mathrm{O}=\mathrm{C}(\mathrm{O}) \mathrm{CCCCCCCC}=\mathrm{CCC}=\mathrm{CCC}=\mathrm{C} \\
\mathrm{CC}\end{array}$ & 6.46 & 7.30 & 0.84 & 6.62 & 0.16 \\
\hline $\mathrm{O}=\mathrm{C}(\mathrm{OCc} \operatorname{ccc} \mathrm{CC}) \operatorname{Cccccc} \mathrm{C}$ & 6.51 & 6.76 & 0.25 & 6.52 & 0.01 \\
\hline $\mathrm{O}=\mathrm{C}(\mathrm{OCCCC}) \operatorname{cccc}(\mathrm{cccc} C$ & 6.51 & 6.76 & 0.25 & 6.61 & 0.10 \\
\hline $\mathrm{O}=\mathrm{C}(\mathrm{O}) \mathrm{CcCC} C \mathrm{CCC}=\mathrm{Cccccc}$ & 6.58 & 6.75 & 0.17 & 6.71 & 0.13 \\
\hline $\begin{array}{l}\mathrm{O}=\mathrm{C}(\mathrm{OCCCCCC}) \mathrm{c}(\mathrm{c}(\operatorname{ccc} 1) \mathrm{C}(=\mathrm{O}) \mathrm{OC} \\
\mathrm{CCCC}) \mathrm{c} 1\end{array}$ & 6.82 & 6.57 & -0.25 & 6.28 & -0.54 \\
\hline $\begin{array}{l}\mathrm{O}=\mathrm{C}(\mathrm{OCCCCCCCC}) \mathrm{c}(\mathrm{c}(\operatorname{ccc} 1) \mathrm{C}(=\mathrm{O}) \\
\mathrm{OCCCC}) \mathrm{c} 1\end{array}$ & 6.82 & 6.57 & -0.25 & 6.47 & -0.35 \\
\hline $\begin{array}{l}\mathrm{O}=\mathrm{C}(\mathrm{OC}) \mathrm{CCCCCCCC}=\mathrm{CCC}=\mathrm{CCCC} \\
\mathrm{CC}\end{array}$ & 6.82 & 7.80 & 0.98 & 6.95 & 0.13 \\
\hline $\begin{array}{l}\mathrm{CCCCCC}=\mathrm{CCC}=\mathrm{CCC}=\mathrm{CCC}=\mathrm{CCCC} \\
\mathrm{C}(=\mathrm{O}) \mathrm{O}\end{array}$ & 6.98 & 8.07 & 1.09 & 6.80 & -0.18 \\
\hline $\begin{array}{l}\mathrm{O}=\mathrm{C}(\mathrm{O}) \mathrm{CCCCCCCC}=\mathrm{CCC}=\mathrm{CCCCC} \\
\mathrm{C}\end{array}$ & 7.05 & 7.51 & 0.46 & 7.06 & 0.01 \\
\hline $\begin{array}{l}\operatorname{cccccccc} C=\operatorname{ccc}=\operatorname{cccccc}(=0) \\
\mathrm{O}\end{array}$ & 7.05 & 7.51 & 0.46 & 7.11 & 0.06 \\
\hline $\mathrm{O}=\mathrm{C}(\mathrm{O}) \mathrm{Ccccccc}$ cсcсcсc & 7.17 & 6.96 & -0.21 & 7.23 & 0.06 \\
\hline $\mathrm{C}(\mathrm{CccccccccccC}) \mathrm{C}$ & 7.20 & 7.22 & 0.02 & 7.70 & 0.50 \\
\hline $\mathrm{O}=\mathrm{C}(\mathrm{OC}) \mathrm{CCC} \operatorname{ccc} \mathrm{cccc}$ & 7.38 & 7.25 & -0.13 & 7.41 & 0.03 \\
\hline $\begin{array}{l}\mathrm{O}=\mathrm{C}(\mathrm{OC}) \mathrm{CCCCCCCC}=\mathrm{CCCCCCC} \\
\mathrm{C}\end{array}$ & 7.45 & 8.02 & 0.57 & 7.94 & 0.49 \\
\hline $\begin{array}{l}\mathrm{Oc}(\mathrm{c}(\mathrm{c}(\mathrm{c}(\mathrm{c} 1) \mathrm{Cl}) \mathrm{Cl}) \mathrm{Cc}(\mathrm{c}(\mathrm{c}(\mathrm{cc} 2 \mathrm{Cl}) \mathrm{Cl}) \mathrm{Cl}) \\
\mathrm{c} 2 \mathrm{O}) \mathrm{c} 1 \mathrm{Cl}\end{array}$ & 7.54 & 6.92 & -0.62 & 6.77 & -0.77 \\
\hline $\begin{array}{l}\mathrm{O}=\mathrm{C}(\mathrm{OCC}(\mathrm{CCCC}) \mathrm{CC}) \mathrm{c}(\mathrm{c}(\operatorname{ccc} 1) \mathrm{C}(=\mathrm{O} \\
) \mathrm{OCC}(\mathrm{CCCC}) \mathrm{CC}) \mathrm{c} 1\end{array}$ & 7.60 & 8.39 & 0.79 & 7.07 & -0.53 \\
\hline $\mathrm{O}=\mathrm{C}(\mathrm{O}) \mathrm{Cccccc} \mathrm{C}=\mathrm{CCCCCCCCC}$ & 7.64 & 7.73 & 0.09 & 7.68 & 0.04 \\
\hline $\mathrm{O}=\mathrm{C}(\mathrm{O}) \mathrm{CcCcCC}=\mathrm{CCCCCCCCCC}$ & 7.64 & 7.73 & 0.09 & 7.69 & 0.05 \\
\hline $\mathrm{Oc}(\operatorname{ccc}(\mathrm{c} 1) \operatorname{ccc} \operatorname{ccc} c \operatorname{ccc}) \mathrm{c} 1$ & 7.91 & 7.46 & -0.45 & 7.81 & -0.10 \\
\hline $\begin{array}{l}\mathrm{O}=\mathrm{C}(\mathrm{OCCCCCCCC}) \mathrm{c}(\mathrm{c}(\operatorname{ccc} 1) \mathrm{C}(=\mathrm{O}) \\
\mathrm{OCCCCCCCC}) \mathrm{c} 1\end{array}$ & 8.10 & 8.54 & 0.44 & 7.76 & -0.34 \\
\hline $\begin{array}{l}\mathrm{O}=\mathrm{C}(\mathrm{OCCCCCCCCCC}) \mathrm{c}(\mathrm{c}(\operatorname{ccc} 1) \mathrm{C}(= \\
\mathrm{O}) \mathrm{OCCCCCC}) \mathrm{c} 1\end{array}$ & 8.10 & 8.54 & 0.44 & 7.91 & -0.19 \\
\hline 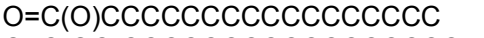 & 8.23 & 7.94 & -0.29 & 8.02 & -0.21 \\
\hline 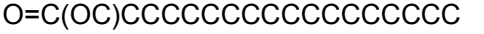 & 8.35 & 8.23 & -0.12 & 8.45 & 0.10 \\
\hline $\mathrm{c}(\mathrm{cccc} 1)(\mathrm{c} 1) \mathrm{CCCCCCCCCCCC}$ & 8.65 & 7.94 & -0.71 & 8.38 & -0.27 \\
\hline 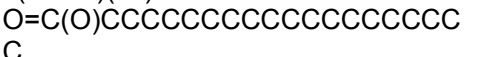 & 9.29 & 8.93 & -0.36 & 8.53 & \\
\hline
\end{tabular}

